\title{
ULTRA LOW POWER ANALOG TO DIGITAL CONVERTER FOR BIOMEDICAL APPLICATIONS
}

\author{
By
}

KARIM ABDELHALIM

\author{
A thesis \\ presented to Carleton University \\ in fulfilment of the \\ thesis requirement for the degree of \\ MASTER OF APPLIED SCIENCE \\ in \\ ELECTRICAL ENGINEERING
}

Ottawa, Ontario, Canada

(C) 2007 Karim Abdelhalim 


$\begin{array}{ll}\begin{array}{l}\text { Library and } \\ \text { Archives Canada }\end{array} & \begin{array}{l}\text { Bibliothèque et } \\ \text { Archives Canada }\end{array} \\ \begin{array}{l}\text { Published Heritage } \\ \text { Branch }\end{array} & \begin{array}{l}\text { Direction du } \\ \text { Patrimoine de l'édition }\end{array} \\ \begin{array}{l}\text { 395 Wellington Street } \\ \text { Ottawa ON K1A ON4 }\end{array} & \begin{array}{l}\text { 395, rue Wellington } \\ \text { Ottawa ON K1A ON4 } \\ \text { Canada }\end{array}\end{array}$

Your file Votre référence ISBN: 978-0-494-33634-2 Our file Notre référence ISBN: 978-0-494-33634-2

NOTICE:

The author has granted a nonexclusive license allowing Library and Archives Canada to reproduce, publish, archive, preserve, conserve, communicate to the public by telecommunication or on the Internet, loan, distribute and sell theses worldwide, for commercial or noncommercial purposes, in microform, paper, electronic and/or any other formats.

The author retains copyright ownership and moral rights in this thesis. Neither the thesis nor substantial extracts from it may be printed or otherwise reproduced without the author's permission.
AVIS:

L'auteur a accordé une licence non exclusive permettant à la Bibliothèque et Archives Canada de reproduire, publier, archiver, sauvegarder, conserver, transmettre au public par télécommunication ou par l'Internet, prêter, distribuer et vendre des thèses partout dans le monde, à des fins commerciales ou autres, sur support microforme, papier, électronique et/ou autres formats.

L'auteur conserve la propriété du droit d'auteur et des droits moraux qui protège cette thèse. $\mathrm{Ni}$ la thèse ni des extraits substantiels de celle-ci ne doivent être imprimés ou autrement reproduits sans son autorisation.
In compliance with the Canadian

Privacy Act some supporting forms may have been removed from this thesis.

While these forms may be included in the document page count, their removal does not represent any loss of content from the thesis.
Conformément à la loi canadienne sur la protection de la vie privée, quelques formulaires secondaires ont été enlevés de cette thèse.

Bien que ces formulaires aient inclus dans la pagination, il n'y aura aucun contenu manquant.

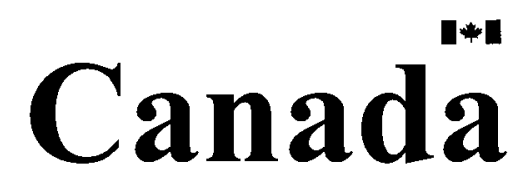




\section{Abstract}

Two 8-bit analog to digital converters (ADCs) were designed for biomedical applications to achieve ultra low power dissipation in a standard $0.13 \mu \mathrm{m}$ CMOS process. Both ADCs make use of the sub-threshold region of operation of the MOSFET to achieve ultra low power dissipation and both ADCs can operate down to a supply voltage of $0.3 \mathrm{~V}$. Both ADCs include an offset correction circuit which significantly reduces the offset voltage as a result of process and mismatch variation. The first ADC, ICGCUADC achieves a measured energy efficiency of $10.3 \mathrm{pJ} /$ cycle operating at $20 \mathrm{kS} / \mathrm{s}$ with a $0.4 \mathrm{~V}$ supply, and achieves a measured ENOB of 7 bits and a SFDR of $53.37 \mathrm{~dB}$ when using a $0.6 \mathrm{~V}$ supply. The second ADC, ICGCUKAH achieves a measured energy efficiency of $8.09 \mathrm{pJ} /$ cycle operating at $20 \mathrm{kS} / \mathrm{s}$ with a $0.4 \mathrm{~V}$ supply, making it the most energy efficient ADC in the published literature to date. The attenuation capacitor is digitally tuned and enables an INL and DNL below 1 LSB,

a measured ENOB of 7.54 bits and a SFDR of $62.2 \mathrm{~dB}$ when using a $0.6 \mathrm{~V}$ supply. 


\section{Acknowledgements}

In the name of God, the Most Gracious, the Most Merciful. All praise and gratitude are for God, the Lord of the Worlds.

I would like to thank my family, including my Dad and Mom, where without them this would be impossible and Amir, Tarek and Vanessa for their love and support.

Foremost, my supervisors Dr. Len MacEachern and Dr. Samy Mahmoud for their support, advice and assistance during this masters degree. Dr. MacEachern offered guidance from when I was in 1st year of my undergraduate degree and has helped me in every way possible to achieve success. Dr. Mahmoud has helped me since I was in late high school, offering support, work and advice during this time.

I would like to thank Nagui Mikhail, who has motivated me and offered advice all through my undergraduate degree. As well he ensured the testing phase of the masters went smoothly and ensured I had all the components and equipment I needed to test my chip.

Next, I would like to thank Ziad El-Khatib, Sinisa Milicevic and Till Kuendiger who helped alot during the tapeout and during the simulation phase of the masters and would not have been possible without them. I would like to thank Vlad Vukovic who designed the PCB for both chips which helped simplify the testing of the ICs. I 
would like to thank Lado Filipovic for soldering all the components to the PCB and assisting in testing my chips. Also, Igor Miletic and Ghyslain Gagnon for helping assisting in analyzing the ADC data. I would also like to thank Zhan $\mathrm{Xu}$, Victor and Vince Karam, Greg Brzezina and Oleksandr Tkachenko for their help during the years of my masters degree.

Also NSERC, NCIT, and CMC for funding the research presented in the thesis. 
to my family ... 


\section{Table of Contents}

Abstract

Acknowledgements iv

Table of Contents vii

List of Tables $\quad \mathbf{x}$

List of Figures $\quad$ xi

$\begin{array}{ll}\text { List of Abbreviations } & \text { xv }\end{array}$

1 Introduction 1

1.1 Motivation . . . . . . . . . . . . . . . . . . . . 1

1.2 Thesis Organization . . . . . . . . . . . . . . . 2

2 Application and Specifications 4

2.1 Application . . . . . . . . . . . . . . . . . 4

2.1 .1 Ultra-low Power Design $\ldots \ldots \ldots \ldots$

2.2 Circuit Specifications . . . . . . . . . . . . . . . . 6

3 Background Material $\quad \mathbf{8}$

3.1 ADC Architecture Survey . . . . . . . . . . . . . . . 8

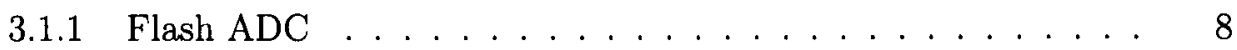

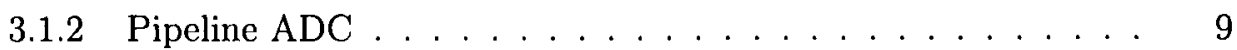

3.1 .3 Sigma-Delta ADC . . . . . . . . . . . . . . . . . 10

3.1 .4 Integrating $\mathrm{ADC} \ldots \ldots \ldots \ldots$

3.1.5 Successive Approximation ADC . . . . . . . . . . . 11

3.2 Published Ultra Low Power ADC Designs . . . . . . . . . . . . . . 13

3.3 Circuit Architecture Selection . . . . . . . . . . . . . . . . 14

3.4 Circuit Components . . . . . . . . . . . . . . . . . 17

3.4 .1 Comparator . . . . . . . . . . . . . . . . 17

3.4 .2 Digital Logic . . . . . . . . . . . . . . . . . . . . . . . . . . 18

3.4 .3 Capacitor Array . . . . . . . . . . . . . . . . 18

vii 
3.4 .4 Switching Network . . . . . . . . . . . . . . . . 18

3.4.5 Switch to Reset Top Plate of Capacitor Array . . . . . . . . . 19

3.4 .6 Output Buffer . . . . . . . . . . . . . . . . . . 19

4 Design and Theory $\quad 20$

4.1 Sub-Threshold Region of a MOSFET . . . . . . . . . . . . . . . . . 20

4.1 .1 Sub-Threshold Analog Operation . . . . . . . . . . . . . 20

4.1.2 Sub-threshold Temperature Effects and Noise . . . . . . . . . 24

4.1.3 Sub-Threshold Digital Operation . . . . . . . . . . . . 26

4.2 System Level of Implemented ADCs . . . . . . . . . . . . . . . . . . . . 27

4.3 Comparator Architecture . . . . . . . . . . . . . . . . . 28

4.3.1 Stage 1 of Comparator . . . . . . . . . . . . . . . . . 28

4.3.2 Monte Carlo Results for Stage 1 . . . . . . . . . . . . . . . 32

4.3.3 Offset Correction Circuitry . . . . . . . . . . . . . . . 33

4.3.4 Comparator Operation . . . . . . . . . . . . . . 35

4.3.5 Monte Carlo Results for Full Comparator . . . . . . . . . . . . 36

4.3.6 Layout for full Comparator . . . . . . . . . . . . . . . 38

4.3.7 Simulation Results for full Comparator . . . . . . . . . . . . . 39

Summary of Bias, Power and Bandwidth . . . . . . . . . . 39

Noise and Temperature . . . . . . . . . . . . . . . . . 42

Comparison of HSPICE and Spectre Simulation Results . . . . 43

4.4 Digital Successive Approximation Register and Control Logic . . . . . 44

4.4 .1 Custom Standard Cell Design . . . . . . . . . . . . . . . . 44

4.4.2 Implementation of SAR and Control Logic . . . . . . . . . . . 46

4.4.3 Layout and Simulation Results for SAR and Control Logic . . 46

Power Dissipation . . . . . . . . . . . . . . . 50

4.5 Capacitor Array . . . . . . . . . . . . . . . . . . . 53

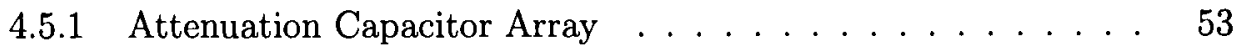

4.5 .2 Tunable Capacitor . . . . . . . . . . . . . . . . 55

4.5 .3 Layout of Capacitor Array . . . . . . . . . . . . . . 56

4.6 Analog Switches, Switch Array and Output Buffers . . . . . . . . . 60

4.6.1 Switch to Reset Capacitor Array . . . . . . . . . . . . . 60

4.6 .2 Analog Switch Array . . . . . . . . . . . . . . . . . 61

4.6 .3 Output Buffers . . . . . . . . . . . . . . . 66

4.7 Analog to Digital Converter Cores . . . . . . . . . . . . . . . . 67

4.7.1 Analog to Digital Converter 1: 8-bit ADC with Full Input Range 67

4.7.2 Analog to Digital Converter 2: 8-bit ADC with Tunable Capacitor Array . . . . . . . . . . . . . . . . . . 70

4.8 Final Analog to Digital Layouts . . . . . . . . . . . . . . . . . . 73

4.8.1 Chip 1: ICGCUADC . . . . . . . . . . . . . . . . 73

4.8 .2 Chip 2: ICGCUKAH . . . . . . . . . . . . 76

viii 
$\begin{array}{lll}5 & \text { Simulations } & \mathbf{7 9}\end{array}$

5.1 Simulation Results of Analog to Digital Converter ICGCUADC . . . 79

5.1 .1 Linearity Results: INL and DNL . . . . . . . . . . . 79

5.1 .2 Dynamic Results: SFDR, SINAD and ENOB . . . . . . . 81

5.1 .3 Power Dissipation . . . . . . . . . . . . . . . . . . . 86

5.1 .4 Temperature. . . . . . . . . . . . . . . . . . 88

5.1.5 Monte Carlo Results . . . . . . . . . . . . . . . . . . . . 89

5.2 Simulation Results of Analog to Digital Converter ICGCUKAH . . 92

5.2 .1 Linearity Results: INL and DNL . . . . . . . . . . . . . 92

5.2 .2 Effect of Tuning on Linearity . . . . . . . . . . . . . . 93

5.2.3 Effect of Tuning on Dynamic Performance . . . . . . . . . . . 94

5.2 .4 Power Dissipation . . . . . . . . . . . . . . . . . . . . . . 95

5.2 .5 Temperature . . . . . . . . . . . . . . . . . . . 97

5.2 .6 Monte Carlo Results . . . . . . . . . . . . . . . . . . . . . 99

5.3 Summary of Simulation Results . . . . . . . . . . . . . . . . . 100

5.3 .1 Application Example . . . . . . . . . . . . . . 101

6 Measurements $\quad 105$

6.1 Measured Results of Analog to Digital Converter 1 . . . . . . . . 105

6.1 Linearity Results: INL and DNL . . . . . . . . . . . . 105

6.1 .2 Dynamic Results: SFDR, SINAD and ENOB . . . . . . 108

6.1 .3 Power Dissipation . . . . . . . . . . . . . . . 110

6.1 .4 Offset Correction . . . . . . . . . . . . . . . 115

6.1 .5 Application Example . . . . . . . . . . . . . . . . . . 117

6.2 Measured Results of Analog to Digital Converter ICGCUKAH . . . . 118

6.2 .1 Linearity Results: INL and DNL . . . . . . . . . . . . 120

6.2 .2 Dynamic Results . . . . . . . . . . . . . . . . . . . 124

6.2 .3 Power Dissipation . . . . . . . . . . . . . . . . . . . . . . . 125

6.2 .4 Application Example . . . . . . . . . . . . . . . 130

6.3 Summary of Measurement Results . . . . . . . . . . . . . . . . 133

6.3 .1 Energy Efficiency . . . . . . . . . . . . . . . . 133

6.3 .2 Accuracy . . . . . . . . . . . . . . . . . 133

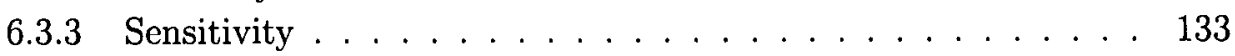

6.4 Comparison to Published Results . . . . . . . . . . . . . . . 134

7 Conclusion 139

7.1 Contributions to Research . . . . . . . . . . . . . . . . . 139

7.2 Publications Resulting from this Work . . . . . . . . . . . . . 141

7.3 Future Work . . . . . . . . . . . . . . . . . . . 141

Bibliography 143 


\section{List of Tables}

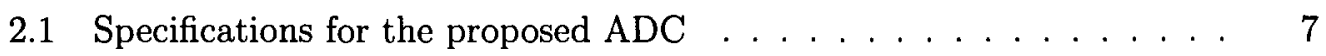

3.1 Other published low power ADC designs. . . . . . . . . . . 14

4.1 Post layout simulation results of bias current, power and bandwidth for comparator. . . . . . . . . . . . . . . . . . 41

4.2 Post layout simulation results of input referred noise for first stage of comparator. . . . . . . . . . . . . . . . . 43

4.3 Post layout simulation results of temperature for comparator. The temperature range where the offset voltage was $-0.5 \mathrm{LSB}$ to $+0.5 \mathrm{LSB}$ was found. . . . . . . . . . . . . . . . 43

4.4 Summary of signals for SAR circuit. . . . . . . . . . . . . . . 48

4.5 Post layout simulation results of $O N$ resistances for the analog switches and their respective settling times for each bit capacitor $V_{D D}=0.4 \mathrm{~V} . \quad 65$

4.6 Summary of ICGCUADC Pins. . . . . . . . . . . . . . . . . . 74

4.7 Summary of ICGCUKAH Pins. . . . . . . . . . . . . . . 77

5.1 Summary of Simulated Results for ICGCUADC . . . . . . . . . . . 102

5.2 Summary of Simulated Results for ICGCUKAH . . . . . . . . . . 102

6.1 Summary of Measured Results for ICGCUADC . . . . . . . . . . . 135

6.2 Summary of Measured Results for ICGCUKAH . . . . . . . . . . . 136

6.3 Comparison to published results. . . . . . . . . . . . . 138

7.1 Comparison between ICGCUADC and ICGCUKAH . . . . . . . 140 


\section{List of Figures}

2.1 Typical block diagram of biomedical transmitter. . . . . . . . . 6

3.1 A flow chart showing the successive approximation algorithm. . . . . 12

3.2 Sampling phase of SAR ADC . . . . . . . . . . . 15

3.3 Phase where the top plate of capacitor array becomes negative $V_{I N}$. 15

3.4 Approximation Phase of SAR ADC . . . . . . . . . . . . 16

3.5 Final phase of SAR ADC, where the digital data is output. . . . . . 16

$4.1 I_{D S}$ vs. $V_{G S}$ for a $V_{D S}$ of $1.2 \mathrm{~V}$ for NFET $\mathrm{W}=2 \mu \mathrm{m}, \mathrm{L}=300 \mathrm{~nm} . \quad \ldots \quad 21$

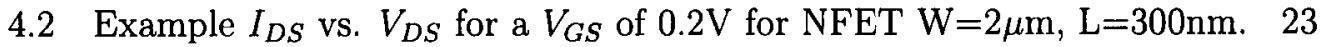

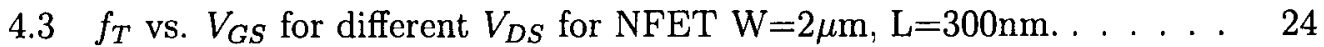

4.4 Capacitance of a standard CMOS transistor from [1] . . . . . . . . . 27

4.5 Circuit Architecture for second ADC, ICGCUADC . . . . . . . . . . 29

4.6 Circuit Architecture for second ADC, ICGCUKAH. . . . . . . . . 30

4.7 Schematic of first stage of comparator circuit with transistor sizes shown. 31

4.8 Monte Carlo Statistical Analysis simulation of 200 runs with process and mismatch variation for the first stage of the comparator. . . . . . 32

4.9 Schematic of full comparator circuit with offset correction. . . . . . . 34

4.10 Waveforms for the comparator preset and reset signals generated by the SAR digital logic. . . . . . . . . . . . . . . . . 36

4.11 Monte Carlo Statistical Analysis simulation of 200 runs with process and mismatch variation for the full comparator. . . . . . . . . . . 37

4.12 Offset voltage shown with and without the offset correction circuit after sweeping the input of the comparator connected to analog ground $\left(V_{\text {tune }}\right) .38$

4.13 Layout of full comparator circuit. . . . . . . . . . . . . . . . 39

4.14 The circuit to bias the comparator. . . . . . . . . . . . . . . 40

4.15 Offset voltages shown when the bias current is swept for a supply voltage of $0.6 \mathrm{~V}$ using HSPICE and Spectre models. . . . . . . . . . . 44

4.16 D-flip flop schematic used for SAR digital circuit. . . . . . . . . . . 45

4.17 Schematic of SAR circuit. . . . . . . . . . . . . . 47

4.18 Schematic of circuit to initialize SAR circuit. . . . . . . . . . . . 48

4.19 Layout of SAR circuit. . . . . . . . . . . . . . . . . . 49

4.20 Input and output signals from SAR circuit. . . . . . . . . . . . 50

4.21 Total digital power dissipation for different supply voltages for clock of $1.2 \mathrm{MHz}, f_{S}=100 \mathrm{kS} / \mathrm{s} . \ldots \ldots \ldots 51$ 
4.22 Total digital power dissipation for different frequencies for a supply of $0.6 \mathrm{~V} \ldots \ldots \ldots \ldots \ldots \ldots \ldots \ldots$

4.23 Monte Carlo Statistical Analysis simulation of 100 runs with process and mismatch variation with a supply of $0.6 \mathrm{~V}$ and a clock of $1.2 \mathrm{MHz}$,

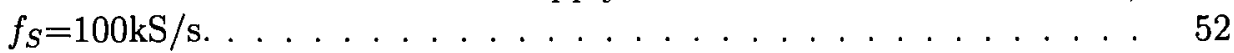

4.24 Binary weighted capacitor array schematic. . . . . . . . . . . . 53

4.25 Binary weighted capacitor array schematic using attenuation capacitor. 54

4.26 Binary weighted capacitor array schematic using tunable attenuation capacitor. . . . . . . . . . . . . . . 56

4.27 Schematic of tunable attenuation capacitor. . . . . . . . . . . 57

4.28 Monte Carlo Statistical Analysis simulation of 200 runs with process and mismatch variation for the tunable capacitor. . . . . . . . . 57

4.29 Layout of capacitor arrays. . . . . . . . . . . . . . . . . . 59

4.30 Switch to GND capacitor array for ICGCUADC. . . . . . . . . . . 62

4.31 Control signals for switch to GND capacitor array $(V D D=0.6 \mathrm{~V}) \ldots \quad 62$

4.32 Schematic of switch array for ADC, ICGCUADC. . . . . . . . . . . 64

4.33 The maximum ON resistances used for the analog switches for different supply voltages. . . . . . . . . . . . . . . . 65

4.34 Schematic of buffer circuit to drive each bit. One buffer circuit is required per bit. . . . . . . . . . . . . 66

4.35 Signals before and after the output circuit. . . . . . . . . . . . . 67

4.36 The layout of ADC core ICGCUADC. The dimensions are $475 \mu \mathrm{m}$ by $390 \mu \mathrm{m} \ldots \ldots \ldots \ldots \ldots \ldots \ldots$

4.37 The signals for the top plate of the capacitor array and control signals for ICGCUADC. . . . . . . . . . . . . . . . . 70

4.38 The layout of ADC core of ICGCUKAH. The dimensions are $475 \mu \mathrm{m}$ by $390 \mu \mathrm{m} \ldots \ldots \ldots \ldots \ldots \ldots \ldots$

4.39 The signals for the top plate of the capacitor array and control signals for ICGCUKAH. . . . . . . . . . . . . . . . . 73

4.40 The final layout of ICGCUADC. Chip dimensions are $1.25 \mathrm{~mm}$ by $1.0 \mathrm{~mm}$, with an area of $1.25 \mathrm{~mm}^{2}$ and a core area of $0.125 \mathrm{~mm}^{2} \ldots 75$

4.41 The final layout of ICGCUKAH. Chip dimensions are $1.25 \mathrm{~mm}$ by $1.0 \mathrm{~mm}$, with an area of $1.25 \mathrm{~mm}^{2}$ and a core area of $0.125 \mathrm{~mm}^{2} \ldots \ldots 78$

5.1 Simulated vout vs. vin of ICGCUADC . . . . . . . . . . . 80

5.2 Simulated INL and DNL of ICGCUADC. . . . . . . . . . . . . 80

5.3 Simulated SFDR and SINAD for ICGCUADC. . . . . . . . . . . 83

5.4 Simulated SINAD when normalized to frequency for ICGCUADC. . . 84

5.5 Simulated ENOB for ICGCUADC. . . . . . . . . . . . 85

5.6 Effective Number of Bits (ENOB) vs. Monte Carlo Iteration for ICGCUADC. 86

5.7 Simulated power dissipation vs. supply voltage for ICGCUADC. . . . 87

5.8 Simulated power dissipation vs. sample rate for ICGCUADC. . . . . 89

5.9 Temperature simulations for ICGCUADC. . . . . . . . . . . . . 90

5.10 Monte Carlo simulations for ICGCUADC. . . . . . . . . . . . . . . 91

5.11 Simulated vout vs. vin curve of ICGCUKAH . . . . . . . . . . 93

xii 
5.12 Simulated INL and DNL of ICGCUKAH. . . . . . . . . . . . 94

5.13 Effect of tuning on DNL of ICGCUKAH . . . . . . . . . . . . 95

5.14 ENOB vs. Input frequency, for each capacitor value for $V_{D D}=0.6 \mathrm{~V}$, $V_{R E F}=0.3 \mathrm{~V}$, sample rate $=60 \mathrm{kS} / \mathrm{s} \ldots \ldots \ldots \ldots$

5.15 Average ENOB $(600 \mathrm{~Hz}$ to $15 \mathrm{KHz})$ from Figure 5.14 for each capacitor value for $V_{D D}=0.6 \mathrm{~V}, V_{R E F}=0.3 \mathrm{~V}$, sample rate $=60 \mathrm{KS} / \mathrm{s} \ldots \ldots \ldots 96$

5.16 Simulated power dissipation vs. supply voltage for ICGCUKAH. . . . 98

5.17 Temperature simulations for ICGCUKAH. . . . . . . . . . . . . . . . 99

5.18 Monte Carlo simulations for ICGCUKAH. . . . . . . . . . . . . . 100

5.19 Simulated output when an analog ECG signal is input into ICGCUADC103

5.20 Simulated output when an analog ECG signal is input into ICGCUKAH104

6.1 Photographs of both ADC chips . . . . . . . . . . . . . . 106

6.2 Scanning electron microscope photograph of the bond wires that connect the PCB to the chip. . . . . . . . . . . . . . . . 106

6.3 Measured digital output versus analog input for ADC ICGCUADC at $60 \mathrm{kS} / \mathrm{s}$ with $\mathrm{Vref}=0.6$ and $V_{D D}=0.6 \mathrm{~V} \ldots \ldots \ldots \ldots \ldots$

6.4 Measured INL and DNL of ICGCUADC. . . . . . . . . . . . . . . 108

6.5 DFT of measured output of ADC ICGCUADC, when input is a 600 $\mathrm{kHz}, 0.6 \mathrm{~V}$ pk-pk Sinusoid. For the ADC, $V_{D D}=V_{R E F}=0.6 \mathrm{~V}$ and sample rate $=60 \mathrm{kS} / \mathrm{s} . \ldots \ldots \ldots \ldots \ldots \ldots \ldots . \ldots \ldots \ldots$

6.6 Measured SINAD and SFDR for ICGCUADC. . . . . . . . . . . 111

6.7 Measured Signal-to-Noise and Distortion (SINAD) vs. normalized frequency when $V_{D D}=V_{R E F}=0.8 \mathrm{~V}, 0.6 \mathrm{~V}, 0.4 \mathrm{~V} \ldots \ldots \ldots \ldots \ldots$

6.8 Measured ENOB for ICGCUADC. . . . . . . . . . . . . . . . . 113

6.9 Measured power dissipation vs. supply voltage for ICGCUADC. . . . 114

6.10 Measured power dissipation vs. sample rate for ICGCUADC for a $0.4 \mathrm{~V}$ and $0.8 \mathrm{~V}$ supply. . . . . . . . . . . . . . . . . . . . 114

6.11 Measured power dissipation for for individual components of ICGCUADC for a $0.6 \mathrm{~V}$ supply. . . . . . . . . . . . . . . . . . . . 116

6.12 Measured Power dissipation of full ADC ICGCUADC vs. sample rate

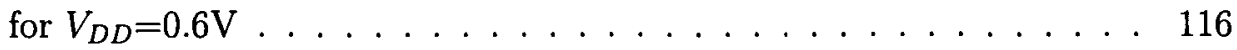

6.13 Negative offset measurements for ICGCUADC. . . . . . . . . . . . 117

6.14 Positive offset measurments for ICGCUADC. . . . . . . . . . . . . . 118

6.15 Measured output when an analog ECG signal is input into ICGCUADC119

6.16 Analysis of measured output when an analog ECG signal is input into ICGCUADC . . . . . . . . . . . . . . . . . . . . . . . . . . . 119

6.17 (a) Photograph of ADC ICGCUKAH. (b) Test setup of ICGCUKAH. 120

6.18 Measured INL and DNL of ICGCUKAH. . . . . . . . . . . . . . . 122

6.19 Measured tuning results for ICGCUKAH for lower capacitor values. . 122

6.20 Measured tuning results for ICGCUKAH for higher capacitor values. 123

6.21 DFT of measured output of ICGCUKAH when input is $1 \mathrm{kHz}$. . . . . 125

6.22 Measured SINAD and SFDR for low capacitor values for ICGCUKAH 126

6.23 Measured SINAD and SFDR for higher capacitor values for ICGCUKAH127

6.24 Measured ENOB for low capacitor values for ICGCUKAH . . . . . . 128 
6.25 Measured ENOB for higher capacitor values for ICGCUKAH . . . . . 129

6.26 Measured average dynamic performance for all capacitor values for ICGCUKAH . . . . . . . . . . . . . . . . . . . . . . . . . 129

6.27 Measured power dissipation vs. sample rate for ICGCUKAH . . . . . 131

6.28 Measured power dissipation vs. supply voltage for ICGCUKAH . . . 132

6.29 Analysis of the measured output when an analog ECG signal is input into ICGCUKAH . . . . . . . . . . . . . . . . . . . 132 


\section{List of Abbreviations}

$\begin{array}{ll}\text { ADC } & \text { Analog-to-Digital Converter } \\ \text { AHDL } & \text { Analog Hardware Description Language } \\ \text { BJT } & \text { Bipolar Junction Transistor } \\ \text { CMOS } & \text { Complimentary Metal Oxide Semiconductor } \\ \text { DAC } & \text { Digital-to-Analog Converter } \\ \text { DC } & \text { Direct Current } \\ \text { DFT } & \text { Discrete Fourier Transform } \\ \text { DNL } & \text { Differential Nonlinearity } \\ \text { ECG } & \text { Electrocardiogram } \\ \text { ENOB } & \text { Effective Number of Bits } \\ \text { ESD } & \text { Electro-Static Discharge } \\ \text { IC } & \text { Integrated Circuit } \\ \text { ICGCUADC } & \text { Chip name for ADC 1 } \\ \text { ICGCUKAH } & \text { Chip name for ADC 2 } \\ \text { INL } & \text { Integral Nonlinearity } \\ \text { LSB } & \text { Least-Significant Bit } \\ \text { MIMcap } & \text { Metal-Insulator-Metal Capacitor } \\ \text { MOSFET } & \text { Metal Oxide Semiconductor Field Effect Transistor } \\ \text { MSB } & \text { Most-Significant Bit } \\ \text { NFET } & \text { N-type Field Effect Transistor } \\ \text { PCB } & \text { Printed Circuit Board } \\ \text { PFET } & \text { P-type Field Effect Transistor } \\ \text { SAR } & \text { Successive Approximation Register } \\ \text { SINAD } & \text { Signal to Noise and Distortion Ratio, (also called SNDR) } \\ \text { SFDR } & \text { Spurious Free Dynamic Range } \\ & \end{array}$

XV 
SMA Sub-Miniature Type A (cable connector)

VDD Positive supply voltage

VDS Drain-Source voltage of a MOSFET

VGS

Gate-Source voltage of a MOSFET

VREF

Reference voltage used by an ADC

VSS

Negative supply voltage

VTC

Voltage Transfer Characteristic

ZVTNFET

Zero-threshold N-type Field Effect Transistors

xvi 


\section{Chapter 1}

\section{Introduction}

\subsection{Motivation}

With an ever-increasing demand for biomedical devices such as pacemakers, hearing aids and biomedical sensor applications, and portable devices such as PDAs and MP3 players, there is more demand for low power analog and digital ICs [2]. An area where energy efficiency is paramount is in circuits in the biomedical field. Implantable chips with bio-sensors are preferably designed to dissipate the minimum power possible since charging or replacing a battery becomes more difficult when its implanted inside the human body.

The analog-to-digital converter $(\mathrm{ADC})$ is a commonly found component in mixed signal integrated circuits such as biomedical implantable chips. A bio-sensor will acquire a biological signal such as blood pressure and convert it into an analog electrical voltage. The ADC will receive the analog information from a bio-sensor and will convert it to digital bits. From here, the bits can be processed and stored and/or transmitted to a signal processor. Typically this ADC uses a significant portion of power for a system which can further decrease the length of time the implantable chip 
can operate inside the human body.

The objective of the research reported in this thesis is to design a fully integrated ADC in a standard CMOS process. The ADC should dissipate only nanowatts of power for bandwidths required for the biomedical field. The goal is to offer state-ofthe-art performance in terms of energy per conversion cycle that compares favourably with results found in the published literature.

\subsection{Thesis Organization}

Chapter 2 provides an overview of the intended application and provides some circuit specifications that will be targeted.

Chapter 3 discusses the different ADC architectures and provides a summary of published low power ADCs. A justification for selecting the successive approximation architecture and the different components that will be designed are briefly discussed.

Chapter 4 presents the design and simulation results for each individual component of the ADC, which includes the comparator, digital logic, capacitor array, analog switches and output buffers.

Chapter 5 presents full post-layout simulation results for two ADC chips designed for this thesis, ICGCUADC and ICGCUKAH, which include static performance, dynamic performance and power dissipation.

Chapter 6 presents test procedures and measurement results for two separate ADC chips designed for this thesis, which include static performance, dynamic performance and power dissipation as well as some ECG measurements.

Chapter 7 concludes the thesis, summarizes the contributions to research and 
provides some suggestions for possible future work. 


\section{Chapter 2}

\section{Application and Specifications}

\subsection{Application}

A typical block diagram of a biomedical transmitter is shown in Figure 2.1. Here a sensor converts a physical measurement such as blood pressure, blood sugar levels or heart signals to an analog voltage level. Typically, the biomedical frequencies of interest can range from $\mathrm{DC}$ to $10 \mathrm{kHz}$ for signals such as electrooculogram (EOG), electroencephalogram (EEG), electrocardiogram (ECG), electromyogram (EMG), and axon action potential (AAP) [3]. The analog signal is amplified and filtered to remove aliasing components and is then passed to an analog-to-digital converter (ADC). The focus is to design such an ADC which will convert the biomedical signal into digital bits. From here digital signal processing (DSP) can be applied and the bits can either be stored in memory to be retrieved at a later time, or transmitted to a nearby digital computer, base station or analog signal processor. The intended application for the ADC described in this thesis is implantable biosensors. Such an application would require minimum power dissipation from implanted circuits as a battery becomes difficult to replace once implanted inside the human body. 
Several authors have proposed that implanted devices derive their power from the host body. Such possible approaches to self-powered systems are described in [4]. One such approach is to use the human body to power an integrated circuit through vibration. It is possible to generate $400 \mu \mathrm{W}$ of power using this technique which is described in [5]. Other techniques for human power generation such as body heat, blood pressure, walking, and movement are described in [6]. These methods of power generation may allow for a batteryless systems but these techniques require ultra-low power circuits using low supply voltages, sub-threshold circuit operation, silicon-oninsulator (SOI) technologies, and multi-threshold CMOS design techniques to ensure that the circuits operate with as low power dissipation as possible. Such ultra low power design techniques are used to design a low-data rate radio as implemented in [7], a low voltage batteryless wireless system as described in [8] and sub-threshold digital logic design to implement a digital filter as described in [9].

\subsubsection{Ultra-low Power Design}

The design of ultra-low power circuits refers to optimizing an integrated circuit such that it performs a computation in the most energy efficient way possible. When designing such integrated circuits everything from the architecture selection to circuit implementation are to be optimized to provide the most energy-efficient solution [10]. Currently, for published 8-bit ADC designs in literature the target energy efficiency for ultra-low power is below $20 \mathrm{pJ} /$ conversion cycle [11], which will be discussed in more detail in the next chapter of this thesis. 


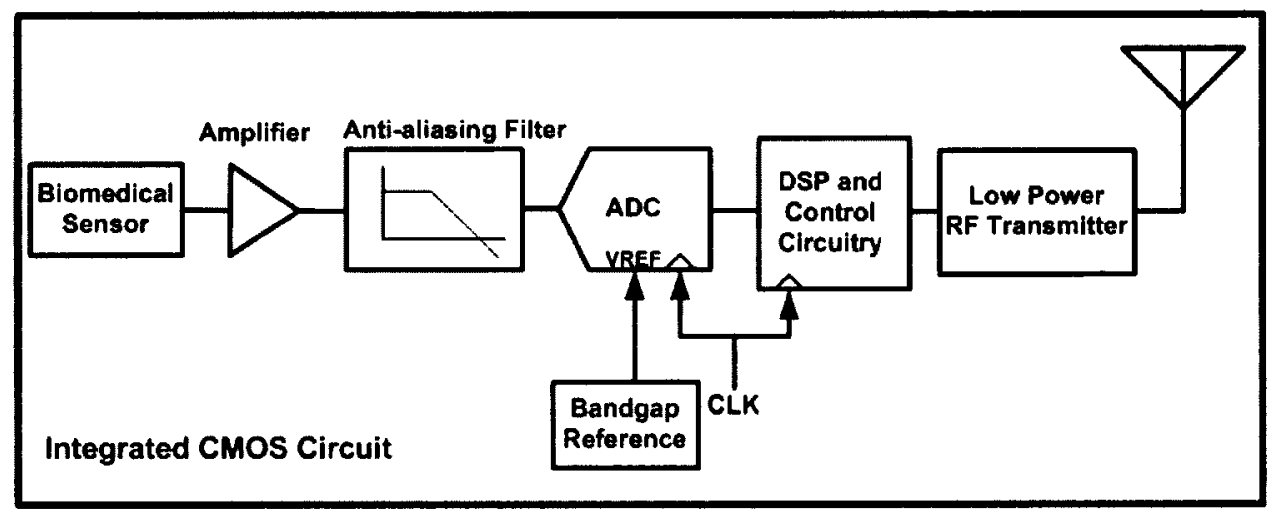

Figure 2.1: Typical block diagram of biomedical transmitter.

The proposed design is to implement an analog to digital converter, using ultralow power design techniques to be used in applications such as implantable chips and other batteryless devices in a standard CMOS process.

\subsection{Circuit Specifications}

The proposed design for the ADC is to use a standard $0.13 \mu \mathrm{m}$ CMOS process with a nominal supply voltage of $1.2 \mathrm{~V}$. The intended ADC targets low to medium sample rates of approximately $1 \mathrm{kS} / \mathrm{s}$ to $1 \mathrm{MS} / \mathrm{s}$ which would provide low data rates for a low-power wireless transmitter. The resolution is to be low to medium with $8-10$ bits of accuracy. Similar applications were found in $[2,11-15]$ to implement medium resolution ADCs (8-10 bits) for low bandwidth signals, such as found in biomedical applications.

Power dissipation is to be among the most important design concerns, with an intended target of less than $1 \mu \mathrm{W}$. The supply voltage is to be as low as possible with a target of half the nominal supply voltage for this process $(0.6 \mathrm{~V})$. Also with using a low supply voltage, the input range should be at least $0.5 V_{D D}$ to $V_{D D}$. For accuracy 
Table 2.1: Specifications for the proposed ADC

\begin{tabular}{|c|c|}
\hline$V_{D D}$ & $0.6 \mathrm{~V}$ \\
\hline Input Range & $0.3-0.6 \mathrm{~V}$ \\
\hline Sample Rate & $1 \mathrm{kS} / \mathrm{S}$ to $1 \mathrm{MS} / \mathrm{s}$ \\
\hline INL & $<1 \mathrm{LSB}$ \\
\hline DNL & $<1 \mathrm{LSB}$ \\
\hline Power & $<1000 \mathrm{nW}$ \\
\hline Area & Minimized \\
\hline Technology & $0.13 \mu \mathrm{m}$ CMOS \\
\hline
\end{tabular}

approximately 8-10 bits for dynamic performance and no missing codes with an INL and DNL of below 1 LSB is expected. The focus of the design will be achieving ultra low power dissipation with minimal trade-offs on accuracy and area. A summary of specifications are included in Table 2.1. 


\section{Chapter 3}

\section{Background Material}

\subsection{ADC Architecture Survey}

\subsubsection{Flash ADC}

The flash ADC is currently the architecture of choice for high-speed applications. The typical design approach is to use a resistor string of equally valued resistors which generate $2^{N}$ reference voltages [16]. Each reference voltage is connected to a comparator, which compares the reference voltage to the analog voltage level. The output from all comparators will be a thermometer code, which will be converted to an N-bit digital output by using a converter [17].

Some advantages of the flash architecture are that the lack of sample and hold circuitry simplifies the hardware required to implement it as it only requires comparators, a resistor network and a decoder. However, one still requires $2^{N}$ comparators which can lead to very high power dissipation and layout area [16]. Also with a large number of comparators connected to the analog input, this can create a large input capacitance for the buffer driving the ADC [18] leading to high power dissipation. This architecture for $8-10$ bits of resolution is not amenable for ultra low power applications. 


\subsubsection{Pipeline ADC}

The concept for the pipeline ADC architecture is to break the conversion up into stages so that the ADC can perform an $\mathrm{N}$-bit conversion in 1 clock cycle, with a latency of N-clock cycles. The operation as described in [17] is as follows; First, the analog signal is sampled and held, and then compared to $V_{R E F} / 2$, where $V_{R E F}$ is a reference voltage chosen. If the analog signal is greater than $V_{R E F} / 2$, this voltage is subtracted from the first stage and then passed on to the next stage. If the analog signal is less than $V_{R E F} / 2$, nothing happens to the analog signal and its passed on to the next stage. If the previous stage output was greater than $V_{R E F} / 2$, the analog signal is multiplied by 2 as it is passed to the next stage.

Each stage contains a sample and hold $(\mathrm{S} / \mathrm{H})$, a comparator, an adder and an amplifier with a gain of 2 [18]. It is also possible to perform more bits per stage which can increase the speed of the conversion cycle or to offer digital correction, such as in [19] which uses 1.5 bits per stage, where 1 bit is for the conversion and 0.5 bits are used for digital correction. Typically the last number of bits will use a small flash ADC to determine the remaining bits for the conversion. Its also important to ensure the first stage is most accurate as the error propagates through the ADC, and the following stages can be less accurate than the stage before it.

The pipeline ADC can operate at high speeds (1 conversion per clock cycle) at a lower area and power dissipation than a flash ADC [16]. The trade-off to this design is the power dissipation as it requires a $\mathrm{S} / \mathrm{H}$, comparator, amplifier, adder per stage $[10]$ which does not make it an ideal candidate for ultra-low power dissipation. 


\subsubsection{Sigma-Delta ADC}

This type of architecture is an oversampling ADC, which requires a sample rate higher than the Nyquist rate *. A typical Delta-Sigma ADC consists of an integrator, a 1-bit $\mathrm{DAC}$ and a 1-bit ADC [16]. The typical approach is to oversample the analog signal and then find the quantization error between the input signal and the output of the DAC. A 1-bit ADC is required which relaxes the analog design components. It is also possible to implement such an ADC with very little silicon area. The main drawback for this application is that it requires fast clocking and filtering which can make it difficult for ultra low power dissipation. Higher-order sigma-delta modulators can be implemented which can further increase the resolution of the ADC [2].

\subsubsection{Integrating $\mathrm{ADC}$}

This type of $\mathrm{ADC}$ consists of an integrator to generate a ramp voltage, a digital counter, and a comparator. The ramp voltage is compared to the analog voltage while the digital circuitry is counting. One can also use a DAC instead of an integrator [20]. This architecture is very slow as it requires $2^{N}$ clock cycles to complete a conversion, but it can be low power, small, and accurate as the analog circuitry is not complex. High resolutions can be obtained by ensuring that the ADC is self-calibrating [20] to ensure comparator offset is minimized and the voltage ramp is properly calibrated to the digital clock. The main drawback for this architecture is the clock, where an 8-bit $\mathrm{ADC}$ sampled at $100 \mathrm{kS} / \mathrm{s}$ would require digital logic to be clocked at $25.6 \mathrm{MHz}$, which can lead to high power dissipation for this ADC.

\footnotetext{
${ }^{*}$ The lowest sampling rate that will permit accurate reconstruction of a sampled analog signal.
} 


\subsubsection{Successive Approximation ADC}

The standard successive approximation architecture contains a comparator, a digital to analog converter (DAC), and digital control logic. The circuit works such that an analog signal is first sampled by the ADC and stored or held. The digital control logic then sets the most significant bit (MSB) to a digital 1 and the rest of the bits to 0 . This represents $V_{R E F} / 2$ and the DAC outputs this value and it is then compared to the analog signal. If $V_{R E F} / 2$ is greater than Vin then the comparator outputs a 0 and the digital logic turns off this bit (MSB). If $V_{R E F} / 2$ is less than $V_{I N}$, then the comparator outputs a 1 , and the digital logic keeps this bit as a 1 . Next clock cycle, we apply a 1 to the next MSB, keeping the rest of the bits as 0 , except the MSB (already determined as a 1 or 0 ). If the MSB was a 1 , then the input into the DAC will be $V_{R E F} / 2+V_{R E F} / 4=3 / 4 V_{R E F}$, and if the MSB was a 0 , then the input into the DAC will be Vref/4. If the output of the DAC is greater than Vin, then a 0 is output from the comparator and this bit is set as a 0 . If the output of the DAC is less than Vin, then a 1 is output by the comparator and this bit is a 1 . So far we can

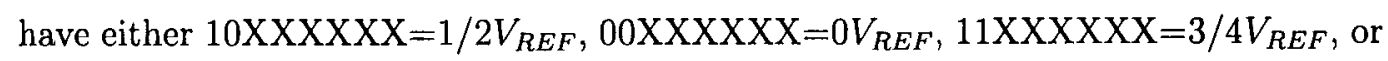

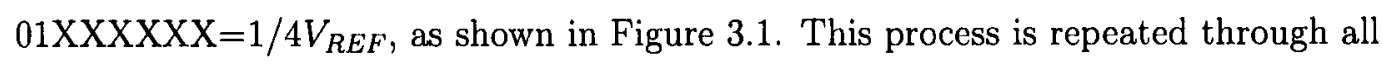
the bits until the DAC output converges to at least 1 LSB compared with Vin.

This architecture has a minimal hardware requirement allowing for low power and can achieve medium speeds where each conversion cycle has the same number of clock cycles as bits which allows the architecture to achieve a high energy efficiency. 


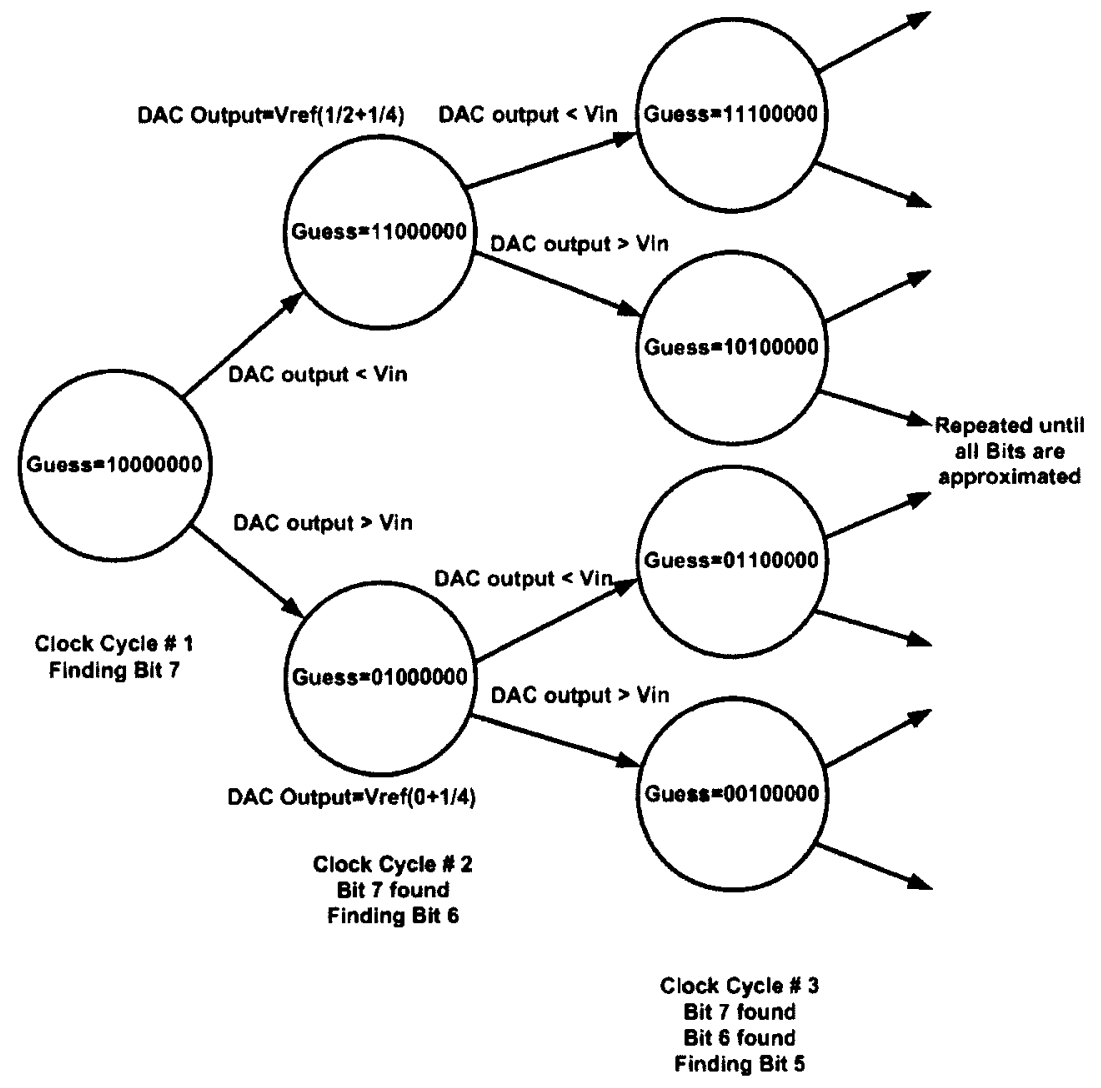

Figure 3.1: A flow chart showing the successive approximation algorithm. 


\subsection{Published Ultra Low Power ADC Designs}

Shown in Table 6.3 is a summary of published measured ultra-low power ADC designs.

The design in [2] uses a successive approximation ADC with charge redistribution to achieve $31 \mathrm{pJ} /$ cycle for an 8 -bit ADC. The ADC design used in [12] uses a similar architecture to achieve low voltage operation $(0.4 \mathrm{~V})$, but it uses an off-chip sample and hold circuit which makes it not fully integrated. The design in [13] makes use of an attenuation capacitor to reduce the size of the capacitor array, which was also implemented in [14]. This design achieves low power dissipation (20pJ/cycle), but makes use of a silicon on saphire process. The lowest power successive approximation ADC using a charge redistribution architecture was found in [21], which uses a $90 \mathrm{~nm}$ CMOS process. This design along with [22] both achieve ultra-low power dissipation by using sub-threshold analog design techniques. Both designs implement 6-bit ADCs.

Other low power designs include integrating ADCs and sigma-delta ADCs. The novel architecture based on an integrating ADC used in [11] achieves low power, small area and uses sub-threshold design techniques. Another integrating ADC found in [23] achieves low power dissipation and high resolution but the sample rates are low and it uses a BiCMOS process. The sigma-delta ADC design in [15] achieves 77pJ per conversion cycle, however this architecture requires additional clocking, and the input signal must be oversampled higher than the Nyquist frequency. 
Table 3.1: Other published low power ADC designs.

\begin{tabular}{|c|c|c|c|c|c|c|c|}
\hline Arch. & Res. & Speed & Power & Tech. & $V_{D D}$ & Area & Energy* \\
\hline SAR [2] & 8-bits & $100 \mathrm{kS} / \mathrm{s}$ & $3.1 \mu \mathrm{W}$ & $0.25 \mu \mathrm{m}$ & $1 \mathrm{~V}$ & $0.053 \mathrm{~mm}^{2}$ & $31 \mathrm{pJ}$ \\
\hline SAR [12] & 8-bits & $4.1 \mathrm{kS} / \mathrm{s}$ & $0.85 \mu \mathrm{W}$ & $0.18 \mu \mathrm{m}$ & $0.5 \mathrm{~V}$ & $0.11 \mathrm{~mm}^{2}$ & $207 \mathrm{pJ}$ \\
\hline SAR [13] & 8-bits & $1 \mathrm{kS} / \mathrm{s}$ & $300 \mathrm{nW}$ & $0.5 \mu \mathrm{m}$ & $2 \mathrm{~V}$ & $\mathrm{~N} / \mathrm{A}$ & $300 \mathrm{pJ}$ \\
\hline Int [11] & 8-bits & $45 \mathrm{kS} / \mathrm{s}$ & $900 \mathrm{nW}$ & $0.18 \mu \mathrm{m}$ & $1.2 \mathrm{~V}$ & $0.021 \mathrm{~mm}^{2}$ & $20 \mathrm{pJ}$ \\
\hline SAR [14] & 8-bits & $409 \mathrm{kS} / \mathrm{s}$ & $7.3 \mu \mathrm{W}$ & $0.5 \mu \mathrm{m}$ & $1.5 \mathrm{~V}$ & $0.481 \mathrm{~mm}^{2}$ & $17.9 \mathrm{pJ}$ \\
\hline SAR [24] & 16-bits & $200 \mathrm{~S} / \mathrm{s}$ & $22 \mu \mathrm{W}$ & $0.18 \mu \mathrm{m}$ & $2 \mathrm{~V}$ & $0.1 \mathrm{~mm}^{2}$ & $110 \mathrm{~nJ}$ \\
\hline SAR [21] & 6-bits & $1.5 \mathrm{MS} / \mathrm{s}$ & $14 \mu \mathrm{W}$ & $0.09 \mu \mathrm{m}$ & $0.5 \mathrm{~V}$ & $0.09 \mathrm{~mm}^{2}$ & $9.33 \mathrm{pJ}$ \\
\hline SAR [22] & 6-bits & $130 \mathrm{kS} / \mathrm{s}$ & $6 \mu \mathrm{W}$ & $0.18 \mu \mathrm{m}$ & $0.65 \mathrm{~V}$ & $0.046 \mathrm{~mm}^{2}$ & $46 \mathrm{pJ}$ \\
\hline$\Sigma \Delta[15]$ & 10-bits & $1.4 \mathrm{MS} / \mathrm{s}$ & $108 \mu \mathrm{W}$ & $0.35 \mu \mathrm{m}$ & $1.8 \mathrm{~V}$ & $\mathrm{~N} / \mathrm{A}$ & $77 \mathrm{pJ}$ \\
\hline Int [23] & 10-bits & $2.9 \mathrm{kS} / \mathrm{s}$ & $17.9 \mu \mathrm{W}$ & $0.8 \mu \mathrm{m}$ & $2.8 \mathrm{~V}$ & $0.8 \mathrm{~mm}^{2}$ & $6.2 \mathrm{~nJ}$ \\
\hline
\end{tabular}

\subsection{Circuit Architecture Selection}

The architecture from $[2,12-14]$ implement a successive approximation architecture based on charge redistribution which offers attractive performance for ultra-low power applications. Other applications such as integrating and $\Sigma-\Delta$ can offer low-power performance, but the oversampling requirements of a $\Sigma-\Delta \mathrm{ADC}$ and the low conversion rate of an integrating ADC ( $2^{N}$ clock cycles) lead to these architectures having higher energy per conversion cycle than the SAR architecture with charge redistribution. These architectures may require less layout area than the SAR architecture with charge redistribution, but the main focus of this thesis work is on an ultra low power dissipation ADC.

This successive approximation architecture based on charge redistribution uses a binary weighted capacitor array as the digital to analog converter needed for the successive approximation ADC. This type of architecture provides low power consumption because it only requires 1 comparator, no sample and hold circuitry, low 


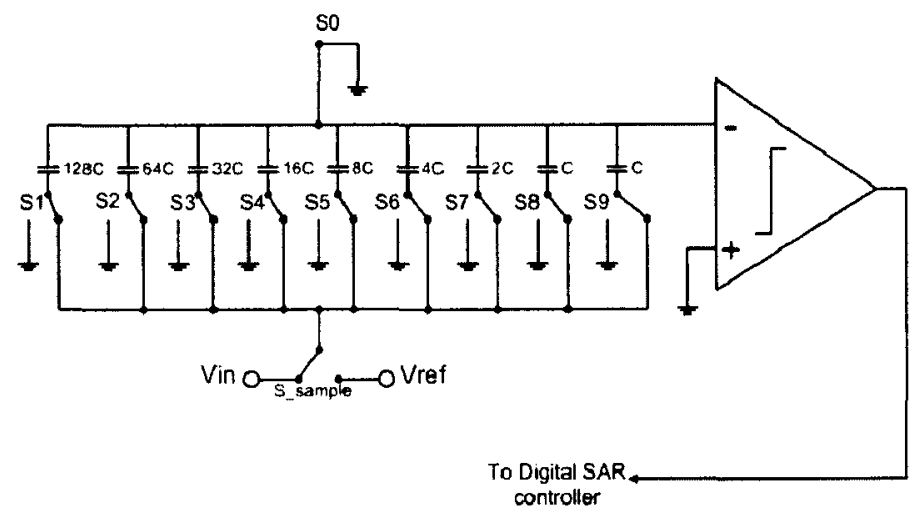

Figure 3.2: Sampling phase of SAR ADC

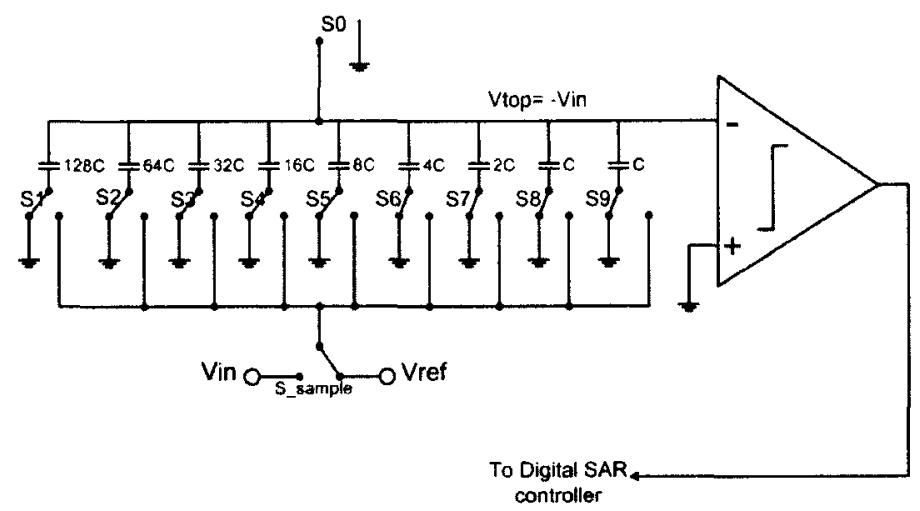

Figure 3.3: Phase where the top plate of capacitor array becomes negative $V_{I N}$.

frequency CMOS digital logic and switching. There are four main phases during the conversion cycle, as described in [25]. First the analog data is sampled onto the bottom plate of the capacitors as $V_{I N}$, while the top plate is grounded as shown in Figure 3.2. Next, the charge is then held as the bottom plate becomes grounded, forcing the top plate to reach negative $V_{I N}$ as shown in Figure 3.3. Next, the approximation stage occurs as in Figure 3.4, where the digital SAR logic controls the switching starting with the most-significant bit (MSB) and the comparator controls whether the voltage is positive or negative at the top plate of the binary weighted capacitor array. If the voltage on the top plate is positive then the approximation was too high, 


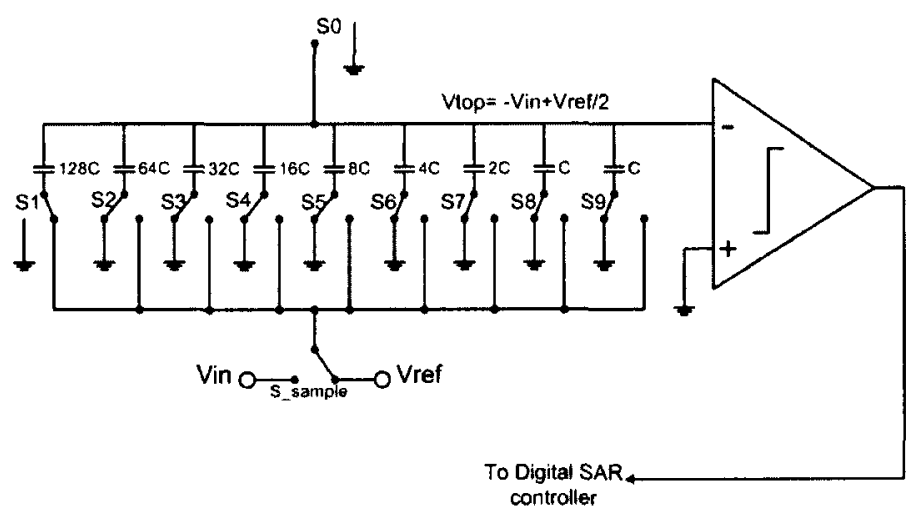

Figure 3.4: Approximation Phase of SAR ADC.

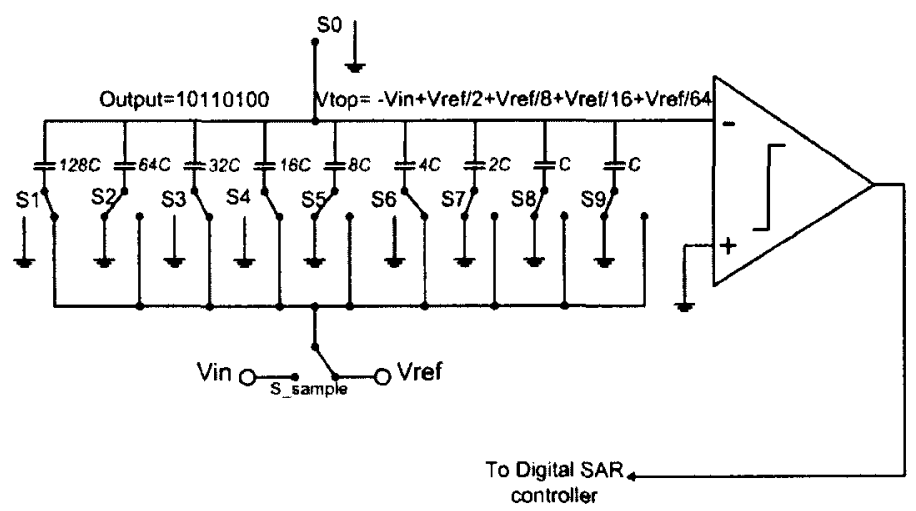

Figure 3.5: Final phase of SAR ADC, where the digital data is output. 
then this bit is a 0 and the top plate is returned to its negative value as before. If the voltage stays negative, then this bit is a 1 and the new negative voltage on the top plate is maintained. This is repeated for each bit, thus the conversion phase will take $n$-clock cycles. After the least-significant bit (LSB) is reached, the digital data is considered to be valid and a new conversion cycle begins, as shown in Figure 3.5. The full conversion should take approximately $n+3$ clock cycles, 1 for sampling the analog signal, 1 for forcing it negative, $n$ for approximating, and 1 for outputting the digital data.

\subsection{Circuit Components}

The successive approximation architecture with charge redistribution is composed of five main components and will be described briefly in the next section and in more detail in the next chapter of this thesis.

\subsubsection{Comparator}

The comparator determines whether the voltage on the top plate of the capacitor array is above or below the analog ground. For an 8-bit design, with a $0.6 \mathrm{~V}$ reference voltage the accuracy of the $\mathrm{ADC}$ should be at least $2.34 \mathrm{mV}{ }^{*}$. The comparator will also contribute the most power dissipation for the entire ADC. To ensure power dissipation is minimized, sub-threshold analog design techniques will be used. The comparator input offset voltage will tend to limit the accuracy of the comparator, and thus common-centroid layout techniques and offset correction will be implemented to minimize the offset voltage of the comparator. Other important factors are circuit

${ }^{*} 0.6 \mathrm{~V} / 2^{8}$ 
noise and temperature effects such as bias shifting which will further reduce the accuracy of the comparator.

\subsubsection{Digital Logic}

This logic implements the successive approximation algorithm using registers and logic gates. The gates are to be implemented using a custom cell library and custom layout to reduce power dissipation. The gates were optimized for noise immunity as $V_{D D}$ will be low $(0.6 \mathrm{~V})$ and low power. It is also important to use separate supply voltages for the analog and digital circuitry, to prevent switching noise from coupling into the sensitive analog components [18].

\subsubsection{Capacitor Array}

The binary-weighted capacitor array implements the charge redistribution concept used in the successive approximation architecture. To minimize capacitor layout area and power dissipation the capacitor array uses an attenuation capacitor as in [13] and [14]. Matching is critical, as mismatch in the capacitors in the array reduces the performance of the ADC [17].

\subsubsection{Switching Network}

An array of analog switches is required to drive each capacitor bit with the reference voltage or GND. The ON resistance of the switch tends to reduce the bandwidth of the ADC. Thus, its important that larger switches will be used for larger capacitors. As the expected analog signals through the switches will be between GND and $V_{D D}$ the switches will be implemented using transmission gates. 


\subsubsection{Switch to Reset Top Plate of Capacitor Array}

To implement an input range from GND to $V_{D D}$, the successive approximation architecture causes voltages to swing below the negative voltage supply. This can cause problems if an NFET switch used as $V_{G S}$ of the transistor will be positive leading to significant leakage on the capacitor array. A PFET switch cannot be used because it cannot pass low voltages well. To solve this problem, a switch was implemented that stores $-V_{D D}$ on a capacitor, then applies it to the gate of a PFET to reset the top plate of the capacitor array to GND.

\subsubsection{Output Buffer}

This will be an 8-bit register followed by large CMOS buffers to drive an off-chip load. A separate buffer supply from the analog and digital ADC circuitry will be used to avoid switching noise being coupled from the large buffers into the ADC.

Each of these five components is further described in more detail in the remainder of the thesis. 


\section{Chapter 4}

\section{Design and Theory}

\subsection{Sub-Threshold Region of a MOSFET}

The sub-threshold operating region of a MOSFET occurs when the MOSFET's gate to source voltage $\left(V_{G S}\right)$ is reduced below the threshold voltage $\left(V_{T H}\right)$ [26]. Shown in Figure 4.1 is the current versus gate-source voltage for an NFET transistor. When $V_{G S}$ is below approximately $0.35 \mathrm{~V}$ in the process technology used in this thesis, the transistor appears to be "OFF" with no drain current present for the NFET device. However, taking the same curve and plotting the current on a log-axis results in the lower plot of Figure 4.1. The increasing straight line when $V_{G S}$ is below $0.35 \mathrm{~V}$ shows that there is a current present and that it is sharply increasing exponentially when compared to the nearly quadratic increase of current when $V_{G S}$ is above the threshold voltage and $V_{D S}$ is greater than $V_{G S}-V_{T H}$.

\subsubsection{Sub-Threshold Analog Operation}

The transistor drain-source current in this region of operation where $V_{G S}$ is below the threshold voltage given by [9], is

$$
I_{D S}=\frac{W}{L} \mu_{\mathrm{eff}} C_{\mathrm{Ox}} v_{T}^{2} \exp \left(\frac{V_{\mathrm{GS}}-V_{\mathrm{TH}}}{m v_{T}}\right)\left(1-\exp \left(\frac{-V_{\mathrm{DS}}}{v_{T}}\right)\right)
$$



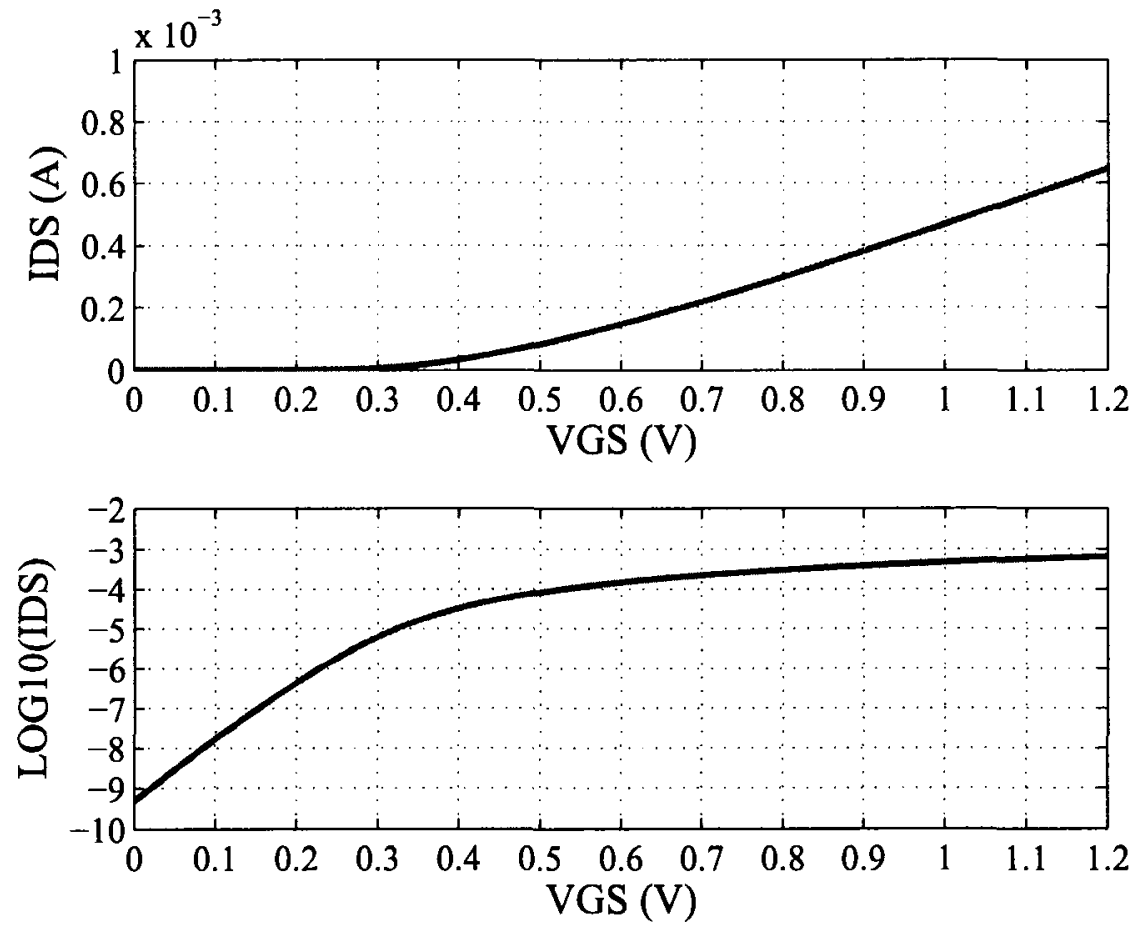

Figure 4.1: $I_{D S}$ vs. $V_{G S}$ for a $V_{D S}$ of $1.2 \mathrm{~V}$ for NFET $\mathrm{W}=2 \mu \mathrm{m}, \mathrm{L}=300 \mathrm{~nm}$. 
where $\mu_{\text {eff }}$ is the effective mobility, $v_{T}$ is the thermal voltage, $C_{O X}$ is the oxide capacitance, $\mathrm{W}$ and $\mathrm{L}$ are the width and length of the MOSFET device respectively, $V_{G S}$ and $V_{D S}$ are the gate-source and drain-source voltages respectively, $m$ is the body effect coefficient given by $1+C_{d} / C_{O X}$ and $V_{T H}$ is the threshold voltage of the MOSFET. This equation shows an exponential relationship with $V_{G S}$ and is similar to the relationship of $V_{b e}$ and $I_{C}$ of a BJT which given by [27] as

$$
I_{c}=I_{s} \exp \left(\frac{V_{B E}}{v_{T}}\right)
$$

This means a MOSFET in the sub-threshold region of operation operates similarly to a $\mathrm{BJT}$ in terms of current versus $V_{G S}$. Also, the sub-threshold current equation as discussed in [9] has the extra exponential term relating it to $V_{D S}$ of the MOSFET. However if $V_{D S}$ is greater than $3 v_{T}$ (75mV at room temperature), the MOSFET's current dependence on $V_{D S}$ is reduced, making it act like a MOSFET in saturation or a $\mathrm{BJT}$ in the active region. It enters the constant current region much earlier than saturation where $V_{D S}$ must be greater than $75 \mathrm{mV}$ instead of $V_{D S}$ being greater than $V_{G S^{-}} V_{T H}$. When $V_{D S}$ is much larger than $V_{T H}$ the current for the sub-threshold MOSFET simplifies to

$$
I_{D S}=\frac{W}{L} \mu_{\mathrm{eff}} C_{\mathrm{OX}} v_{T}^{2} \exp \left(\frac{V_{\mathrm{GS}}-V_{\mathrm{TH}}}{m v_{T}}\right)
$$

This constant current region is shown in Figure 4.2 where $V_{G S}$ is set to $0.2 \mathrm{~V}$ and the NFET transistor is in the sub-threshold region. This characteristic of the subthreshold region of the MOSFET allows for analog design for very low supply voltages and currents to be feasible. Analog components such as current mirrors, amplifiers and active loads can be designed similarly as if one was designing devices using BJTs 


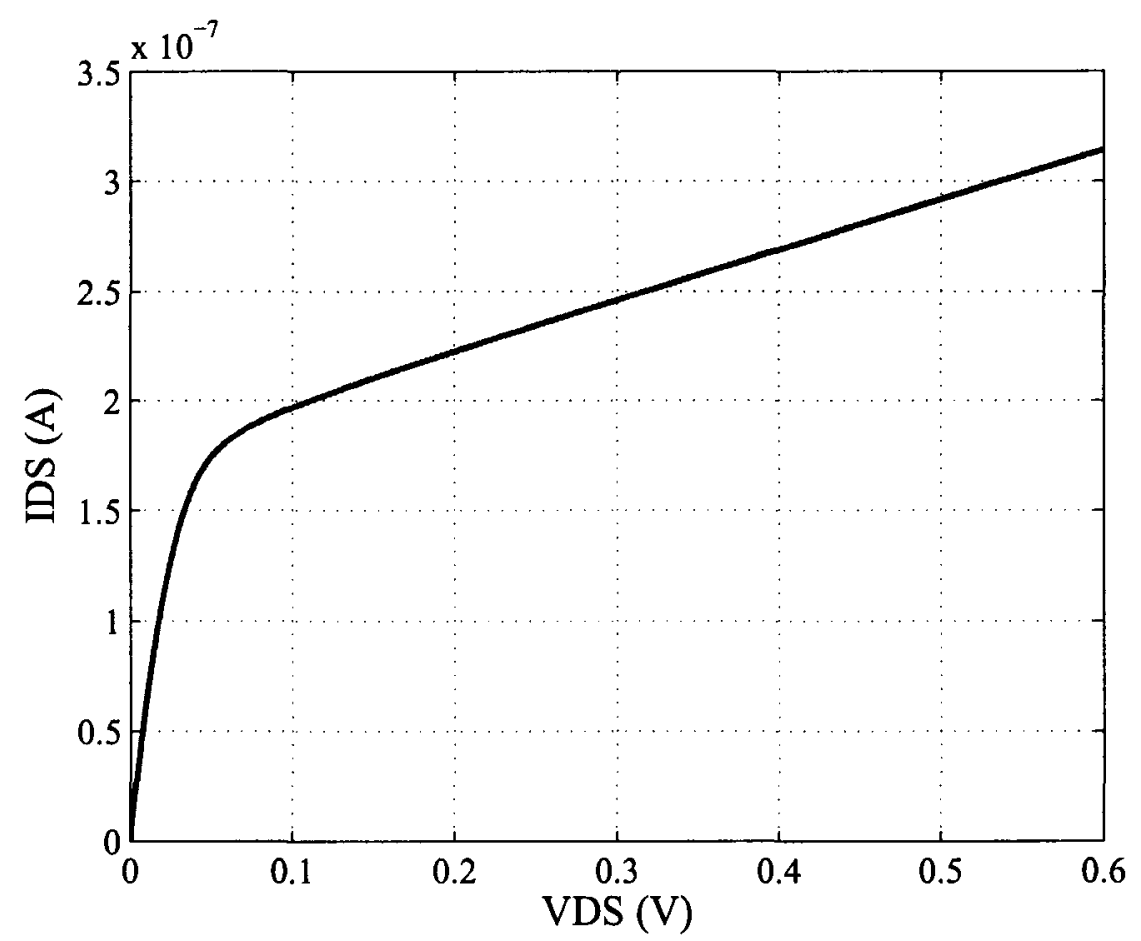

Figure 4.2: Example $I_{D S}$ vs. $V_{D S}$ for a $V_{G S}$ of $0.2 \mathrm{~V}$ for NFET $\mathrm{W}=2 \mu \mathrm{m}, \mathrm{L}=300 \mathrm{~nm}$. or MOSFETs operating in active region or saturation region respectively. The tradeoff however becomes the frequency of operation as operating in sub-threshold has low currents which results in a very low $g_{m}$ for the device. In [17] $g_{m}$ in sub-threshold is $I_{D} /\left(n V_{T}\right)$ which means it increases linearly with drain current while in saturation $g_{m}$ increases with the root of the drain current. The equation for $f_{T}$ of the transistor, given in [17] is

$$
f_{T}=\frac{g_{m}}{2 \pi C_{G S}}
$$

Operating the device in sub-threshold with a low $g_{m}$ value will lead to a much lower $f_{T}$ than operating the transistor in saturation mode. Shown in Figure 4.3 is an NFET device of $\mathrm{W}=2 \mu \mathrm{m}$ and $\mathrm{L}=300 \mathrm{~nm}$ operating in sub-threshold has $f_{T}$ plotted versus 


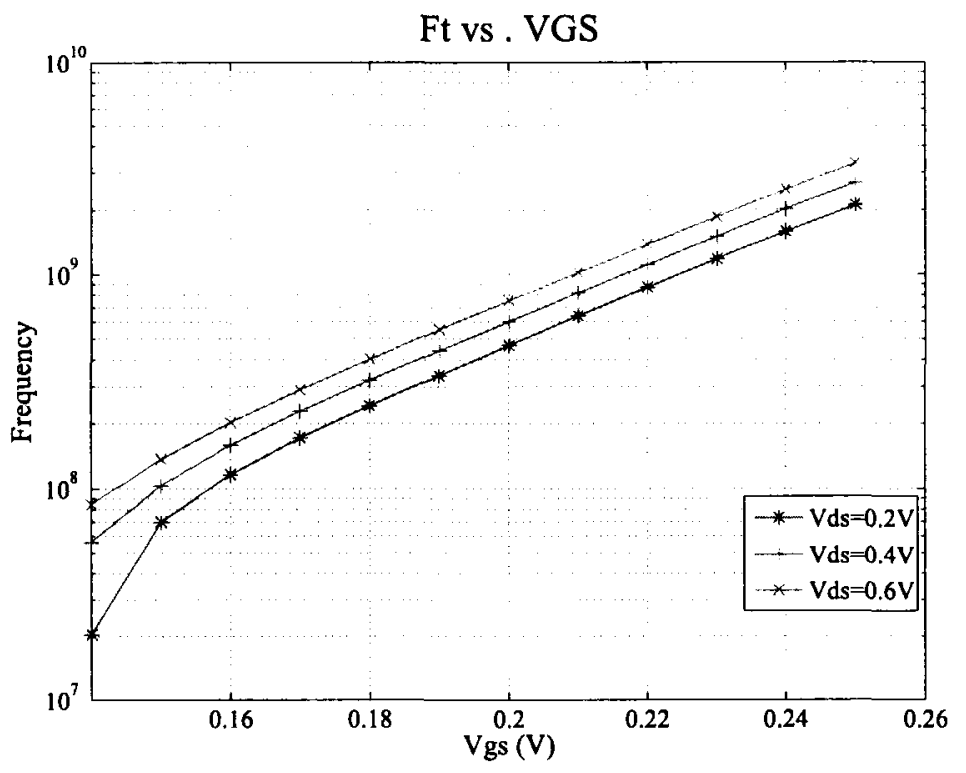

Figure 4.3: $f_{T}$ vs. $V_{G S}$ for different $V_{D S}$ for NFET $\mathrm{W}=2 \mu \mathrm{m}, \mathrm{L}=300 \mathrm{~nm}$.

$V_{G S}$ for 3 different $V_{D S}$ values. The $f_{T}$ which is in the $\mathrm{MHz}$ and low $\mathrm{GHz}$ range is significantly lower than found when operating the same device in saturation mode, where the $f_{T}$ is approximately $100 \mathrm{GHz}$.

\subsubsection{Sub-threshold Temperature Effects and Noise}

The threshold voltage and mobility both decrease with an increasing temperature where at low $V_{G S}$ the decrease in voltage threshold dominates which leads to an increase in drain current ${ }^{*}[17]$. An increase in temperature will cause the off-current $\left(I_{O F F}\right)$ to increase quadratically and the sub-threshold slope (S) to increase linearly [1]. This will lead to higher leakage currents for digital logic and higher drain currents for analog design, with an overall effect of increasing total power dissipation of the system.

\footnotetext{
${ }^{*}$ Opposite holds true for higher $V_{G S}$.
} 
Since operation in the sub-threshold region of operation is typically low bandwidth the main concern will be $1 / f$ noise, which results from the trapping of charges at the oxide/semidconductor interface [17]. In the saturation region of operation of the MOSFET, the input-referred $1 / f$ noise given in $[28]$ is,

$$
S_{V g}=\frac{q^{2} N_{O T}}{C_{O X}^{2} W L f}
$$

where $I_{d}$ is the drain current, $C_{O X}$ is the oxide capacitance, $W$ and $L$ are the width and length of the gate respectively, $N_{O T}$ is the equivalent energy of oxide traps. In the sub-threshold region of operation of the MOSFET there is the presence of capacitive ratio between the oxide capacitance $\left(C_{O X}\right)$ and the depletion capacitance $\left(C_{d}\right)$ where the input-referred $1 / f$ noise in sub-threshold given in [28] is,

$$
S_{V g}=\left(\frac{C_{i n v}}{C_{O X}}\right)^{2} \frac{q^{2} N_{O T}}{n^{2} C_{O X}^{2} W L f}
$$

where $n$ is $\left(C_{O X}+C_{d}\right) / C_{O X}, W$ and $L$ are the width and length of the gate respectively. The capacitor divider ratio allows the $1 / \mathrm{f}$ input-referred noise to be lower in subthreshold than in saturation [28]. The drain current noise power spectrum density is determined in [28] by multiplying the input-referred noise by $g_{m}^{2}$. This results in a drain current noise power spectrum density in sub-threshold to be quadratically related to $I_{d}$ and inversely proportional to $C_{o x}$ and the area of the transistor gate [28]. Thus by ensuring that the area of transistor gates are large noise can be reduced [22] for MOSFETs operating in sub-threshold. 


\subsubsection{Sub-Threshold Digital Operation}

For standard static CMOS logic implemenations, operating the devices in sub-threshold can provide a more ideal voltage transfer curve (VTC) than compared to operating the devices in saturation. This is because as the drain-source voltage decreases the current also decreases when in saturation mode, while in sub-threshold the current is approximately constant, all the way down to when $V_{D S}$ is approximately $v_{T}[9]$. As mentioned in [9] the sub-threshold static CMOS logic operation is more robust compared with saturation static CMOS logic when normalized to $V_{D D}{ }^{*}$. It was found in [9] that sub-threshold pseudo-NMOS logic can offer a twenty percent improvement over sub-threshold static-CMOS in terms of delay and power but at the expense of reduced noise margins. Another advantage of sub-threshold operation for digital logic is the lower input capacitance. As shown in Figure 4.4, the depletion capacitance forms a series capacitance with $C_{O X}$. This reduces the input capacitance, and the main contributor to the input capacitance becomes the overlap capacitances of the gate. This becomes important for total power consumption, as the power of standard CMOS logic is given by [9],

$$
P=\alpha C_{L} V_{D D}^{2} f+I_{l e a k} V_{D D}
$$

where $\alpha$ is the probability of the gate switching, $C_{L}$ is the load capacitance, $f$ is the frequency, and $I_{\text {leak }}$ is the static current flow when the device is not switching. When operating in sub-threshold mode, with $V_{D D}$ and the input capacitance being significantly lower than when operating in saturation mode, one can achieve ultra

\footnotetext{
${ }^{*}$ Has higher noise margins when normalized to $V_{D D}$.
} 


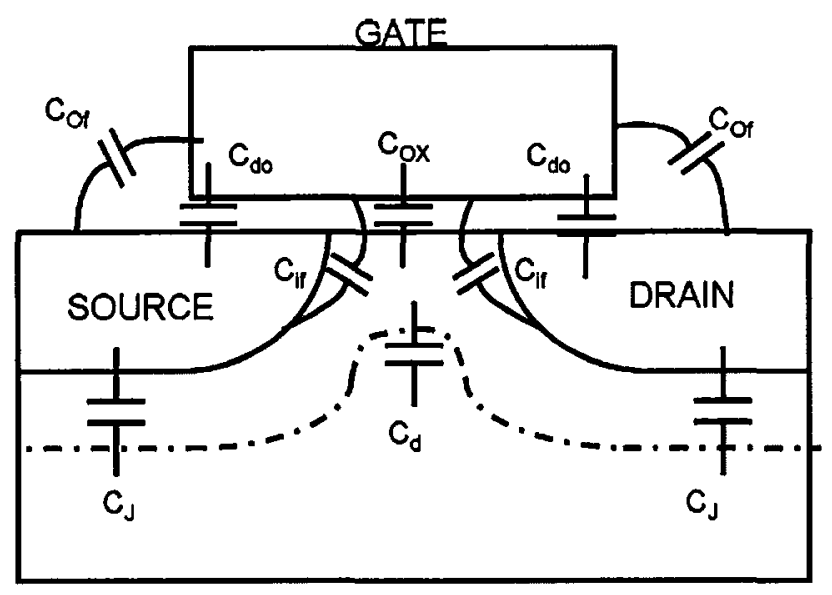

Figure 4.4: Capacitance of a standard CMOS transistor from [1]. $C_{O X}$ is the oxide capacitance, $C_{d}$ is the depletion capacitance, $C_{d o}$ is the overlap capacitance, $C_{j}$ is the junction capacitance and $C_{i f}$ and $C_{o f}$ are fringing capacitance.

low power dissipation for digital logic, when high speed operation is not the main concern.

\subsection{System Level of Implemented ADCs}

Two ADCs were designed. Both designs implement 8-bit successive approximation ADCs using charge redistribution and make use of the sub-threshold region of operation of the MOSFET, employ similar techniques to remove the offset voltage for the comparator and make use of an attenuation capacitor to reduce the area and power of the capacitor array, where it splits the standard binary weighted capacitor array into two smaller capacitor arrays with a series capacitor between them. The first ADC, ICGCUADC shown in Figure 4.5 makes use of a low leakage switch to allow the ADC to have a full input range as it uses $\mathrm{OV}$ as the analog GND and uses zero threshold input transistors for the comparator. The second ADC, ICGCUKAH shown in Figure 4.6 has one half of the input range compared to the first ADC with an analog 
GND of $V_{D D} / 2$, but makes use of a 3-bit digitally tuned capacitor to implement the attenuation capacitor and uses NFET input transistors for the comparator.

\subsection{Comparator Architecture}

Two comparators where designed. One comparator uses zero-threshold NFETs at the input of the comparator for the ADC that uses $0 \mathrm{~V}$ as the analog ground, and the other comparator uses standard NFETs at the inputs of the comprator for the ADC that uses $V_{D D} / 2$ as the analog ground. The former comparator is described next.

\subsubsection{Stage 1 of Comparator}

The first stage of the comparator shown in Figure 4.7 uses a differential amplifier. It uses active PMOS loads and a current mirror to set a constant current in subthreshold. Long channel devices were used for the load and current mirror transistors to ensure a higher gain. The input transistors were made minimum length for the zero-threshold transistors of this process, and made wide with multiple fingers to improve device matching.

The $V_{\text {tune }}$ node in Figure 4.7 was connected to analog ground in this work, but could be used to tune for any residual input offset voltages. The current mirror transistor M5 is biased with a $V_{G S}$ low enough that it is in deep sub-threshold mode but high enough so that it is still accurately modeled with minimum variation between simulation and measured as reported in the process documentation. This was determined to be as low as approximately $0.15 \mathrm{~V}$ for $V_{G S} . \mathrm{M} 5$ is the output side of a sub-threshold current mirror which is independent of $V_{D S}$ with currents in the 


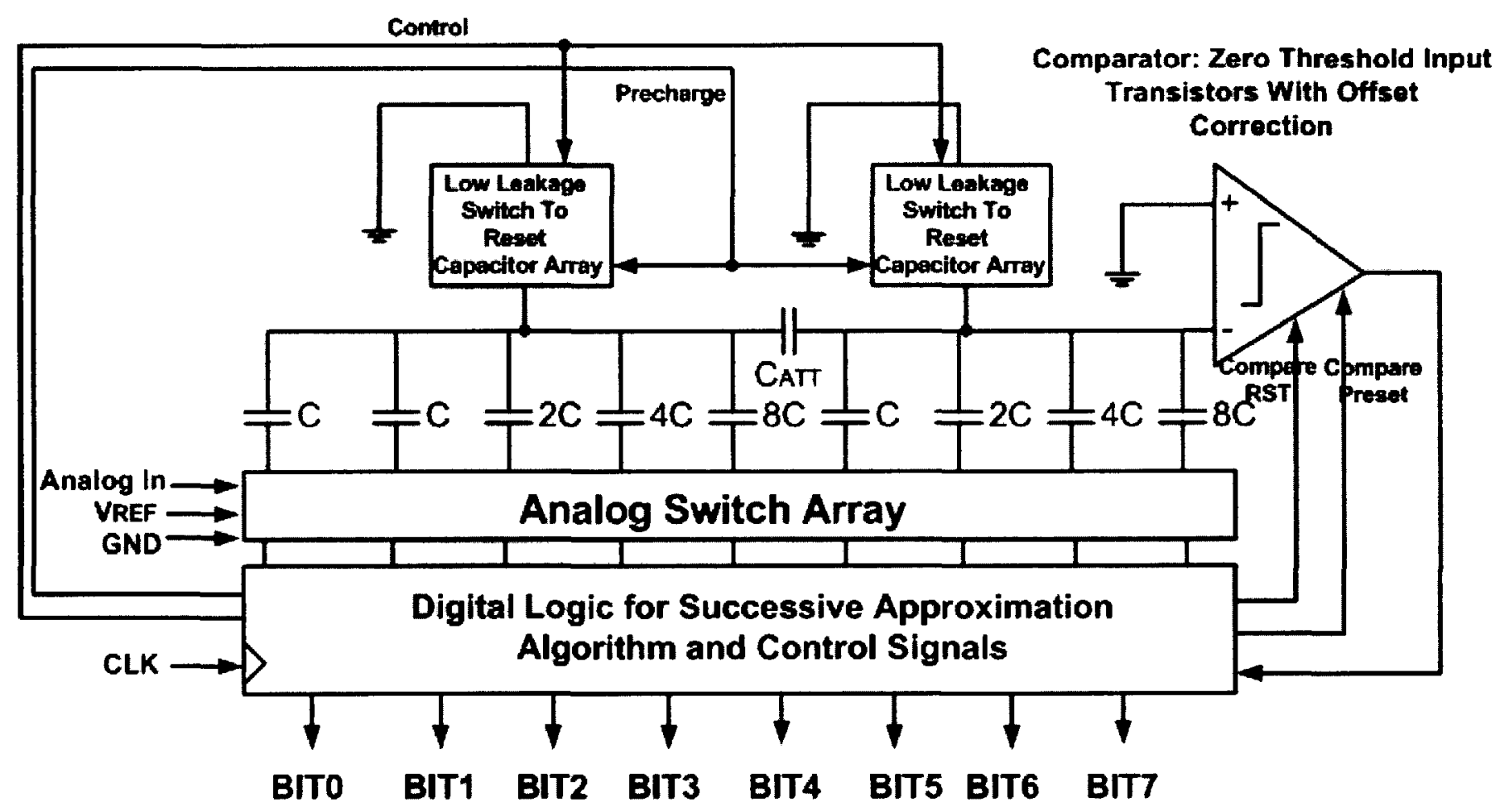

Figure 4.5: Circuit Architecture for first ADC, ICGCUADC. The main circuit components are the digital logic for the successive approximation algorithm, an analog switch array, low leakage switches to reset the top plate of the capacitor array, a differential zero-threshold NFET comparator, and a capacitor array which uses an attenuation capacitor (output buffers and registers are not shown). Signals include the digital outputs which are labeled Bito..Bit7, CLK which is used for the timing of the digital circuitry, GND which is the analog GND which is OV for this ADC, $V_{R E F}$ which is the reference voltage where analog input signals are between $G N D$ and $V_{R E F}$, Analog In which is the analog signal into the ADC between $G N D$ and $V_{R E F}$, Control and Precharge which are signals generated by the digital circuitry to control the switches to reset the top plate of the capacitor array, Compare RST and Compare Preset which are generated by the digital circuitry to control the offset correction for the comparator (The digital circuitry, low leakage switches, analog switch array and comparator all have a positive supply voltage and negative supply voltage $\left(V_{D D}\right.$ and $\left.G N D\right)$. 
Comparator: NFET Input

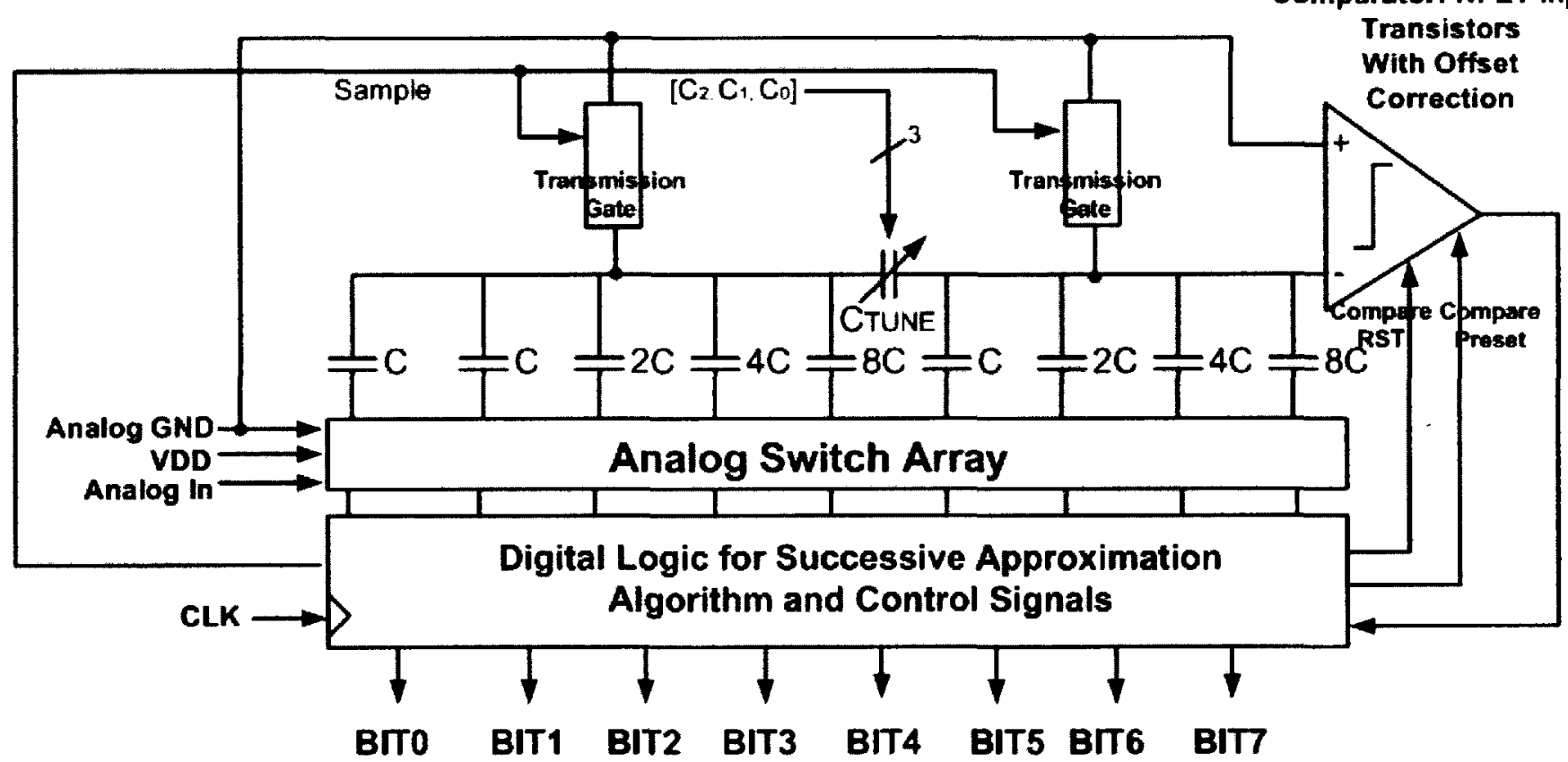

Figure 4.6: Circuit Architecture for second ADC, ICGCUKAH. The main circuit components are the digital logic for the successive approximation algorithm, an analog switch array, transmission gates to reset the top plate of the capacitor array, an NFET differential comparator, and a capacitor array which uses a 3-bit digitally tuned attenuation capacitor (output buffers and registers are not shown). Signals include the digital outputs which are labeled Bit0..Bit7, CLK which is used for the timing of the digital circuitry, analog GND which is the analog ground which is $V_{D D} / 2$ for this ADC, $V_{D D}$ which is the reference voltage where the analog input signals are between analog GND and $V_{D D}$, Analog In which is the analog signal into the ADC between analog GND and $V_{D D}$, Sample which resets the top plate of the capacitor array, $C 2 C 1 C 0$ is a 3-bit digital input to tune the attenuation capacitor, Compare RST and Compare Preset which are generated by the digital circuitry to control the offset correction for the comparator. (The digital circuitry, transmission gates, the tunable capacitor, analog switch array and comparator all have a positive supply voltage and negative supply voltage ( $V_{D D}$ and $\left.G N D\right)$. 


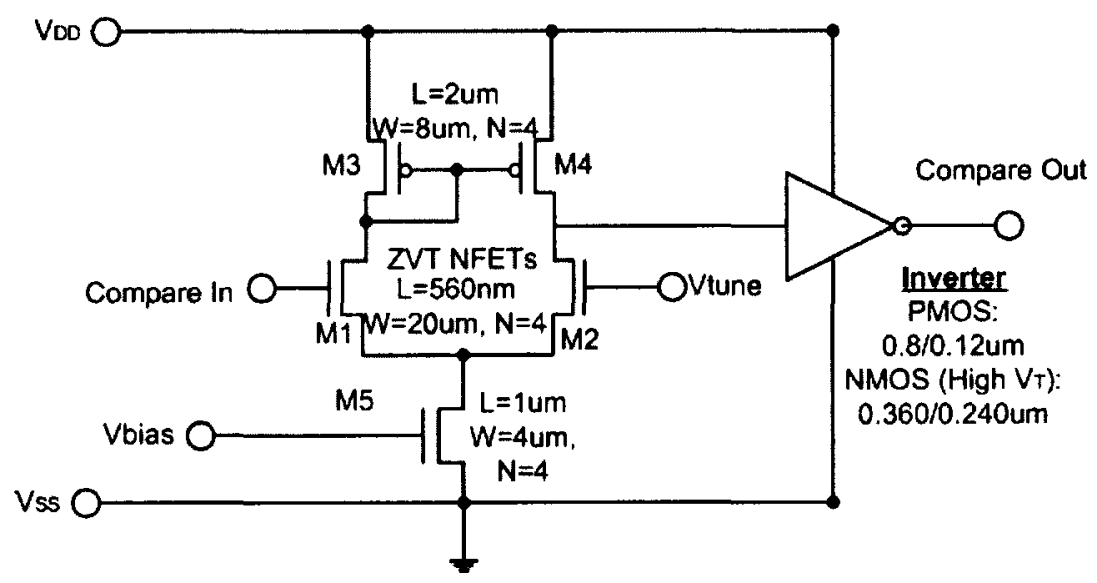

Figure 4.7: Schematic of first stage of comparator circuit with transistor sizes shown. nano-ampere range.

When the input at the compare_in signal goes above analog ground, the input transistor M1 passes the majority of the bias current bringing the output high as well. Similarly, when the input to the compare_in signal goes below analog ground, M1 is essentially shut off, and the majority of current will be routed through transistor M2 connected to the $V_{\text {tune }}$ node bringing the output low. In [18] a small signal analysis determines the gain of the first stage to be,

$$
A_{v}=g_{m}\left(r_{O U T} \| \frac{1}{s C_{L}}\right)
$$

where $r_{\text {OUT }}$ is the output impedance from transistors M4 and $\mathrm{M} 2, C_{L}$ is the capacitive load of the next stage plus the output capacitance of transistors M4 and M2 and $g_{m}$ is the sub-threshold transconductance for device M2 *.

\footnotetext{
${ }^{*}$ Given from [17] as $\frac{I_{Q}}{n V_{T}}$
} 


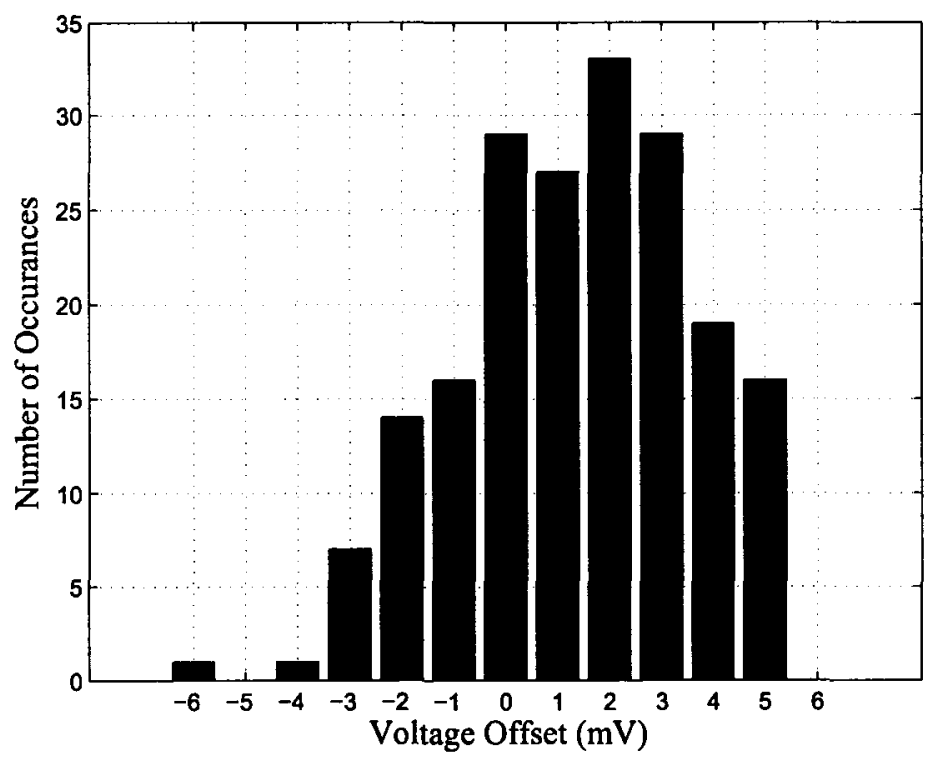

Figure 4.8: Monte Carlo Statistical Analysis simulation of 200 runs with process and mismatch variation for the first stage of the comparator.

\subsubsection{Monte Carlo Results for Stage 1}

The main disadvantage of using the circuit in Figure 4.7 is that it will have an offset voltage from mismatch variations of the transistors. After running a Monte Carlo Statistical Analysis simulation using process and mismatch models, with 200 runs *, the resulting offset voltage is determined to have a mean offset of $1.88 \mathrm{mV}$ with a standard deviation of $2.2 \mathrm{mV}$ as shown in Figure 4.8. For an 8-bit ADC with a $0.6 \mathrm{~V}$ supply, a voltage accuracy of $2.34 \mathrm{mV}$ for the LSB is required. The offset must be below this value so that the LSB is not affected but this is not the case as was determined from the Monte Carlo simulation.

*Determined to be a statistically significant sample size based on documentation for Spectre and HSPICE. 


\subsubsection{Offset Correction Circuitry}

The offset voltage as a result of process and mismatch variation degrades the accuracy of the comparator. Typical methods of offset correction are avoided in low power designs as they increase the overall power consumption and can be done off-chip by digital signal processing [2]. A technique to remove the offset voltage was to use the circuit from Figure 4.7 as a pre-amplifier and the offset voltage from this circuit was stored on a capacitor during the reset phase and a similar differential amplifier with NFET input transistors was then used for a second stage. The circuit is shown in Figure 4.9. During the sampling phase of the ADC the two inputs, $V_{T U N E}$ and Compare In of the comparator are set to analog ground. This will force the output of the first stage to be set to some nominal output, which is approximately $V_{D D} / 2$. As seen in Figure 4.8 there is a significant first stage output offset voltage due to process and mismatch variation on the transistors. The output of the first stage during the sampling phase is therefore $V_{D D} / 2$ plus an additional offset voltage which will be stored on the capacitor shown in Figure 4.9.

During the conversion phase, the second stage has one input transistor connected to the capacitor with the stored output voltage from stage 1 , and the other input transistor connected to the output of stage 1 . The differential second stage removes the offset voltage from stage 1 . There will be an offset voltage for stage 2 , but because the signal has already been amplified from the gain of the first stage the accuracy of the second stage can be relaxed ${ }^{*}$.

${ }^{*}$ Alternatively, the second stage offset is less important because the desired signal has been amplified. 


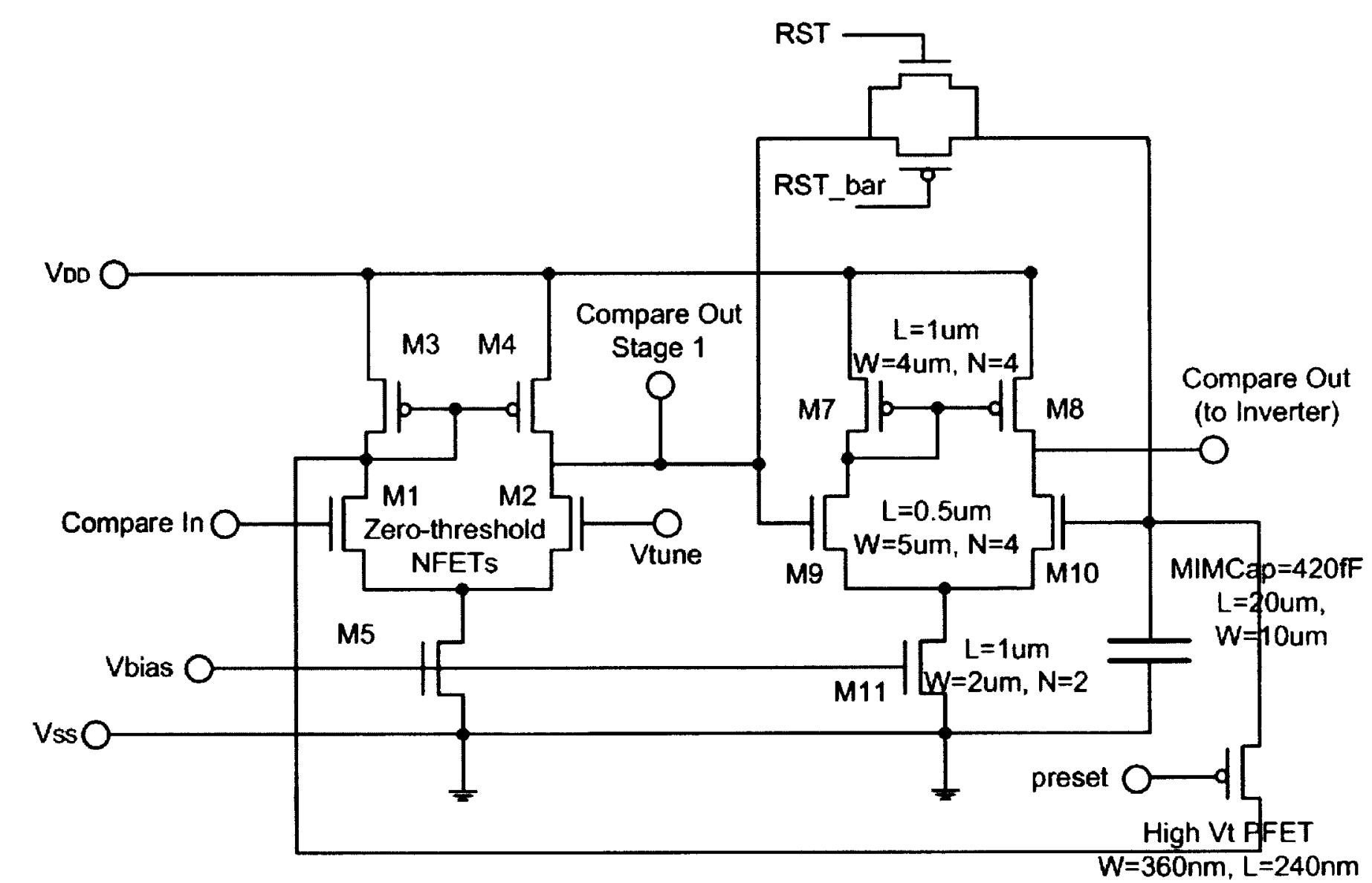

Figure 4.9: Schematic of full comparator circuit with offset correction. 
It was found that by charging the capacitor in Figure 4.9 to GND or to $V_{D D}$ before storing the offset voltage, this would significantly reduce the speed of the comparator especially when operating in sub-threshold. Also charging the capacitor to the output of the first stage would not be practical as the voltage at the input of the comparator will be positive or negative, and the output of the first stage will be much higher or lower than the nominal output voltage. This also would require time to charge the capacitor to the required voltage to remove the offset from stage 1. A solution to the problem is to pre-charge the capacitor to the node connected to the gates of the PFET transistor, M3 in the active loads of stage 1 . This voltage is nearly identical to the output voltage of stage 1 when no offset is present in the circuit and both inputs are the same. Thus, this gives a good approximation of what to charge the capacitor to before sampling the offset output from stage 1.

\subsubsection{Comparator Operation}

The comparator shown in Figure 4.9 works as follows. First, the comparator pre-charge signal shown in Figure 4.10 goes low which occurs at the end of a conversion cycle. This will turn on the PMOS device, which is a high threshold transistor to minimize leakage from the capacitor. When the PMOS device is ON, the voltage from the drain of transistor M3 from the first stage is connected to the capacitor. This will pre-charge the capacitor to approximately $V_{D D} / 2$. Next, the comparator reset signal shown in Figure 4.10 goes low at the same time the ADC is sampling the analog signal. Thus, the inputs to the comparator are both connected to analog ground. 

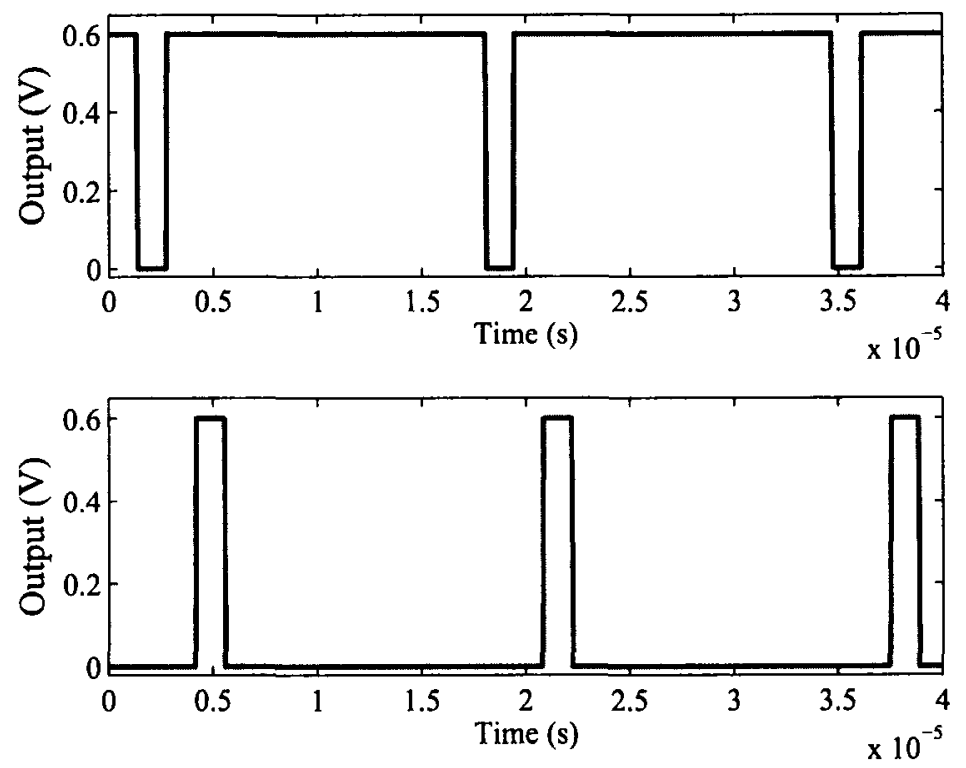

Figure 4.10: Waveforms for the comparator preset and reset signals generated by the SAR digital logic.

The output voltage of the first stage of the comparator gets connected to the capacitor through a transmission gate, and the capacitor will charge (or discharge) from approximately $V_{D D} / 2$ to $V_{D D} / 2+A_{v}$ off set. Once the comparator reset goes low the conversion cycle begins with one input of stage 2 connected to the output of stage 1 and the other input of stage 2 connected to the capacitor, with the resulting effect of the offset of stage 1 being minimized.

\subsubsection{Monte Carlo Results for Full Comparator}

After running a Monte Carlo Statistical Analysis simulation using process and mismatch models, with 200 runs, the mean offset voltage was $-233 \mu \mathrm{V}$ with a standard deviation of $487 \mu \mathrm{V}$. The mean offset was reduced by 8 times and the standard deviation was reduced by 4.5 times when using the offset correction circuitry. This offset 


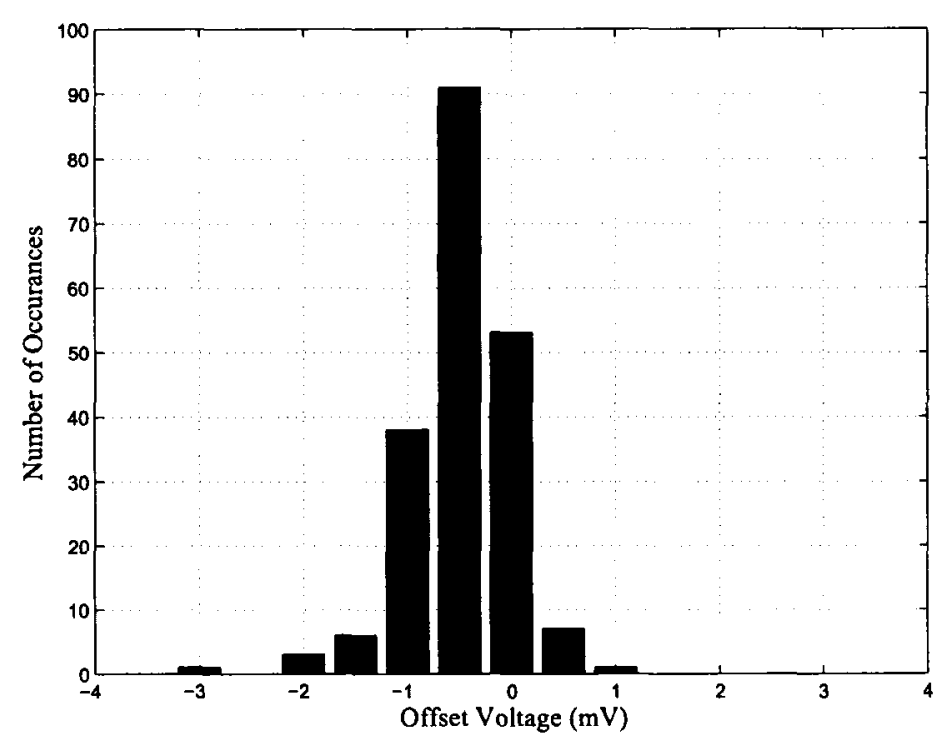

Figure 4.11: Monte Carlo Statistical Analysis simulation of 200 runs with process and mismatch variation for the full comparator.

correction improves the accuracy of the comparator, and should allow it to provide 8-bits of resolution for the implemented ADC after fabrication. It is also important to note that the previous case had 8 out of 200 trials that had an offset voltage greater than $10 \mathrm{mV}$, (not shown in Figure 4.8), while all 200 cases for the offset corrected comparator had all offsets below $10 \mathrm{mV}$.

Shown in Figure 4.12 the input $V_{\text {tune }}$ which is analog ground in this thesis was swept from $-6 \mathrm{mV}$ to $+6 \mathrm{mV}$. This introduced a comparator voltage offset which can be modeled as an input referred offset voltage for the comparator [17]. Looking at the extremes in Figure 4.12, a $-6 \mathrm{mV}$ input offset produces a $850 \mu \mathrm{V}$ offset for the comparator with offset correction and $-4.6 \mathrm{mV}$ with no offset correction, and for a $6 \mathrm{mV}$ input offset it produces a $490 \mu \mathrm{V}$ offset with offset correction and $7.7 \mathrm{mV}$ without offset correction. Figure 4.12 demonstrates that the offset correction circuitry reduces the 


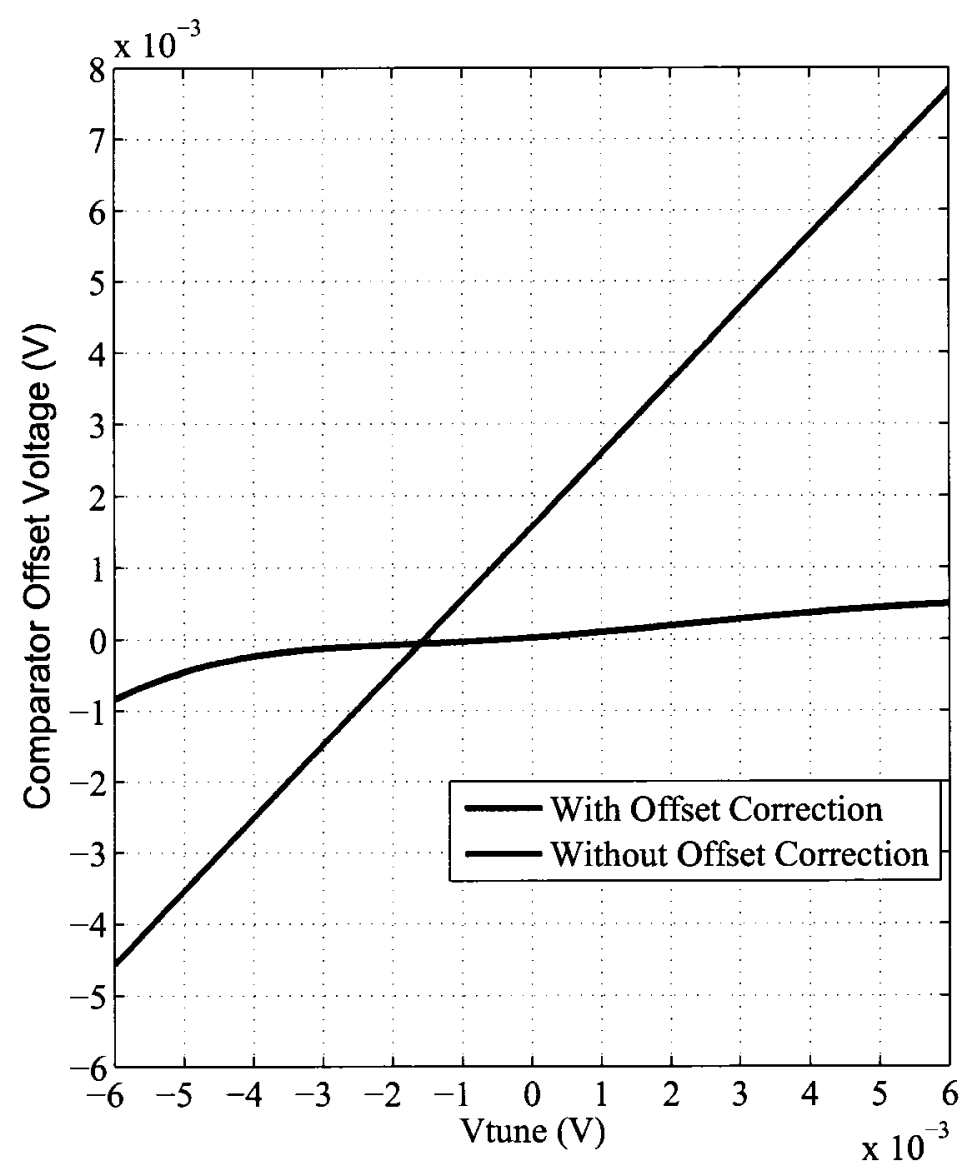

Figure 4.12: Offset voltage shown with and without the offset correction circuit after sweeping the input of the comparator connected to analog ground $\left(V_{\text {tune }}\right)$.

input referred offset voltage irrespective of whether it is positive or negative.

\subsubsection{Layout for full Comparator}

The layout of the full comparator schematic of Figure 4.9 is shown in Figure 4.13 with dimensions of $90 \mu \mathrm{m}$ by $45 \mu \mathrm{m}$. The active PMOS loads and input transistors for both stages used a common centroid layout orientation to improve matching. The NFET input differential pair transistors, the zero-threshold NFET input different pair transistors, the PMOS active load transistors, the bias NFET transistor and the high 


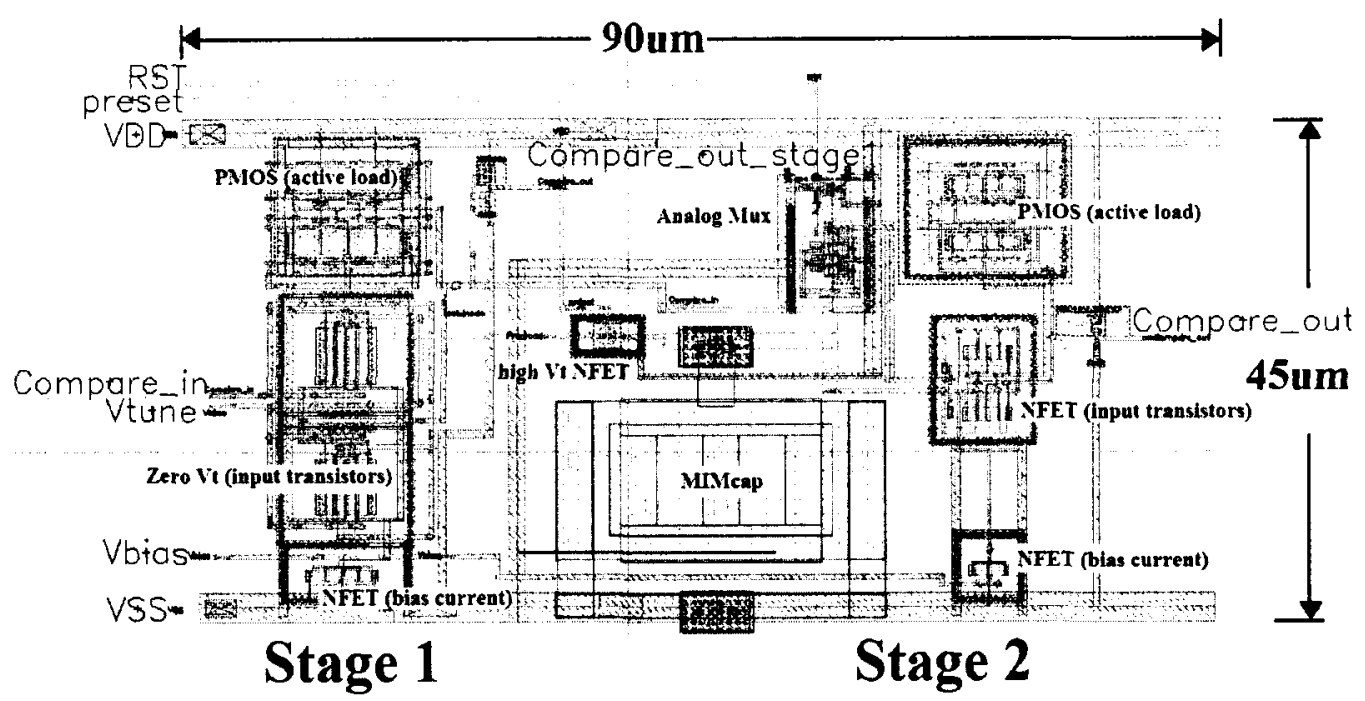

Figure 4.13: Layout of full comparator circuit.

threshold NFET transistor were each placed in a guard ring for isolation from digital components on the chip. The entire comparator was also placed in a guard ring with an $\mathrm{N}$-well (not shown in Figure 4.13) to further minimize noise injection from digital circuits elsewhere on the chip [18].

\subsubsection{Simulation Results for full Comparator Summary of Bias, Power and Bandwidth}

The circuit used to generate $V_{\text {bias }}$ is shown in Figure 4.14. It is a diode connected transistor with a $\mathrm{W} / \mathrm{L}$ of $(2 \mu / 1 \mu)$ using a 1:2 ratio to save power. In Table 4.1, the minimum current that allowed for a $-250 \mu \mathrm{V}$ offset voltage and the maximum current that allowed for a $250 \mu \mathrm{V}$ offset voltage is shown. Any bias current between these two extremes set the comparator offset voltage to be below $250 \mu \mathrm{V}$. In the deep subthreshold regime as shown in Table 4.1 , for a supply voltage of $0.3 \mathrm{~V}$ and $0.4 \mathrm{~V}$, the current has a smaller range to keep the offset reduced. For the cases with a supply 


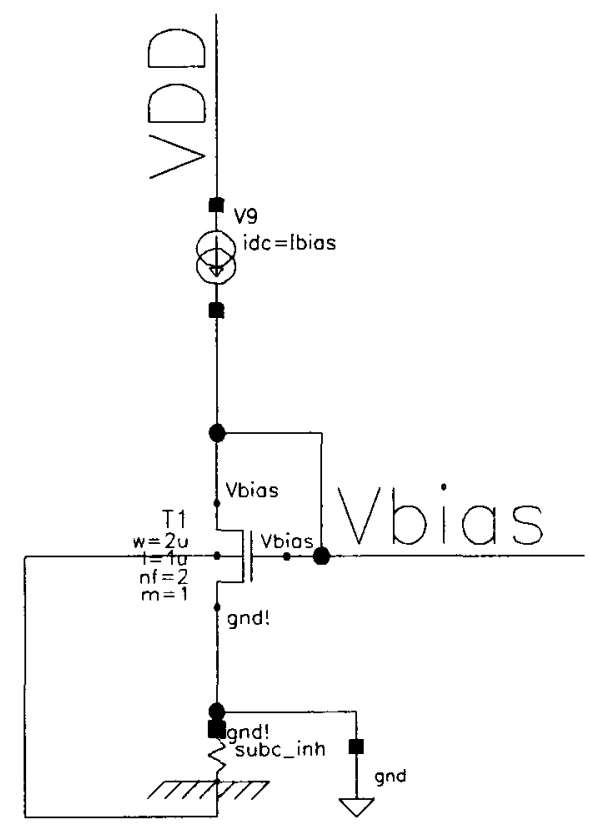

Figure 4.14: The circuit to bias the comparator.

of $0.5 \mathrm{~V}$ or higher, the bias current could vary more and a low offset voltage was still achievable. The bandwidth and power dissipation are also shown in Table 4.1 for the case when using the minimum bias current. The lowest power dissipation observed occurs when operating in the deep sub-threshold region where the comparator can achieve $88 \mathrm{kHz}(8 \mathrm{kS} / \mathrm{s})$ bandwidth while dissipating only $30 \mathrm{nW}$. The highest power dissipation occurs when a higher supply is used where the comparator achieves $3.3 \mathrm{MHz}(300 \mathrm{kS} / \mathrm{s})$ bandwidth while dissipating $2.37 \mu \mathrm{W}$. A higher bias current allows for more bandwidth at the expense of power dissipation. For the ADC the charging and discharging of the capacitor array will be the bottleneck for speed, not the comparator, thus the lowest bias current should be used to bias the comparator to minimize power dissipation. It is also important to note that for all the cases in Table 4.1 the comparator is biased in the sub-threshold regime where $V_{\text {bias }}$ is between $0.1 \mathrm{~V}$ 
Table 4.1: Post layout simulation results of bias current, power and bandwidth for comparator.

\begin{tabular}{|c|c|c|c|}
\hline$V_{D D}(\mathbf{V})$ & Ibias (nA) & Power (nW) & Bandwidth (MHz) \\
\hline 0.3 & $20-50$ & 30 & 0.088 \\
\hline 0.4 & $40-150$ & 65 & 0.22 \\
\hline 0.5 & $125-880$ & 234 & 0.66 \\
\hline 0.6 & $304-4500$ & 636 & 1.3 \\
\hline 0.7 & $607-11000$ & 1370 & 2.2 \\
\hline 0.8 & $968-19500$ & 2370 & 3.3 \\
\hline
\end{tabular}

to $0.25 \mathrm{~V}$ for supplies between $0.3 \mathrm{~V}$ and $0.8 \mathrm{~V}$ respectively.

The main bottleneck for bandwidth of the comparator is the charging of the offset capacitor through a transmission gate where the bandwidth will be [17],

$$
\begin{aligned}
& B W=0.7 \frac{1}{R_{N} \| R_{P} C} \\
& B W=0.7 \frac{1}{R_{N} \| R_{P} 0.5 p F}
\end{aligned}
$$

where $R_{N}$ and $R_{P}$ are the ON resistances of the NFET and PFET in the transmission gate respectively and $\mathrm{C} \quad 0.5 \mathrm{pF}$ is the offset correction capacitor. The resistance lowers inversely with $V_{D D}$ and $\mathrm{W}$ of the transistor. Thus, with lower supply voltages the bandwidth reduces significantly. The bandwidth of the actual differential amplifiers is given by [17],

$$
B W=\frac{1}{r_{01} \| r_{02} C_{L}}
$$

where $r_{01}$ and $r_{02}$ are the output resistors of the zero threshold input NFETs and the PMOS active load respectively, and $C_{L}$ is the input capacitance of the second stage NFET transistors plus the output capacitance of the 1st stage. 


\section{Noise and Temperature}

A post layout noise simulation was performed on the first stage of the comparator and its results are summarized in Table 4.2. The comparator noise analysis is difficult because of the non-linear behavior of the circuit as well as the different phases of the comparator [10]. Noise on the input of the comparator can lead to errors at the output, and thus it is important that the input-referred noise is minimized such that it is less than 1 LSB [10]. The main contributor to noise was the active load and input transistors. Wide transistors helped reduce noise for the input transistors [17] and a large gate for the active load transistors also reduced the noise.

Table 4.2 shows simulated input referred noise for the first stage of the comparator. When the simulated comparator noise at the output is normalized to 1 LSB (assuming 8-bit resolution) it is shown that for a minimum current that allows the comparator to function properly the highest input-referred noise was $0.032 \mathrm{LSB}$ when a $V_{D D}$ of $0.3 \mathrm{~V}$ was used and the lowest input-referred noise was $0.016 \mathrm{LSB}$ when a $V_{D D}$ of $0.8 \mathrm{~V}$ was used. The comparator output noise is lower than the offset voltage of the comparator when process and mismatch variation is introduced $(250 \mu \mathrm{V})$ and when mismatch of the capacitor array is added, noise of the comparator becomes insignificant for this 8-bit ADC.

Table 4.3 shows the effect of temperature on the offset voltage of the comparator. When the comparator operates in deep sub-threshold $\left(V_{D D}=0.3-0.4 \mathrm{~V}\right)$ the comparator has a much smaller temperature range in which it can function properly. This is largely due to sensitivity of the bias current when the comparator operates with 
Table 4.2: Post layout simulation results of input referred noise for first stage of comparator.

\begin{tabular}{|c|c|c|c|}
\hline$V_{D D}(\mathrm{~V})$ & Sample Rate $(\mathrm{kS} / \mathrm{s})$ & Noise $(\mu \mathrm{V})$ & Noise (LSB) \\
\hline 0.3 & 8 & 39 & 0.032 \\
\hline 0.4 & 20 & 41 & 0.026 \\
\hline 0.5 & 60 & 42 & 0.022 \\
\hline 0.6 & 120 & 42 & 0.018 \\
\hline 0.7 & 200 & 44 & 0.016 \\
\hline 0.8 & 300 & 51 & 0.016 \\
\hline
\end{tabular}

Table 4.3: Post layout simulation results of temperature for comparator. The temperature range where the offset voltage was -0.5LSB to +0.5LSB was found.

\begin{tabular}{|c|c|}
\hline$V_{D D}(\mathbf{V})$ & Temperature Range $\left({ }^{\circ} \mathrm{C}\right)$ \\
\hline 0.3 & $17.4-41.4$ \\
\hline 0.4 & $-16.5-60$ \\
\hline 0.5 & $<79.8$ \\
\hline 0.6 & $<105$ \\
\hline 0.7 & $<117$ \\
\hline 0.8 & $<123$ \\
\hline
\end{tabular}

low supply voltages. Its also important to note that the power dissipation of the comparator also increases with temperature, as the current in the sub-threshold region is proportional to $v_{T}$. When using supply voltages less than $0.4 \mathrm{~V}$, the comparator can still be used in implantable biomedical applications as the human body temperature stays within the simulated temperature range of the comparator.

\section{Comparison of HSPICE and Spectre Simulation Results}

The comparator is operating in the sub-threshold mode. It becomes important to ensure the models are working accurately. One technique was to ensure the sizes of the transistors and $V_{G S}$ of the transistors are operating in a region that is well correlated between models and measurement provided by the process documentation. Another precaution was to simulate the comparator using BSIM3.3 and BSIM4 models using both Spectre and HSPICE and ensure that results all agree closely with each other. 


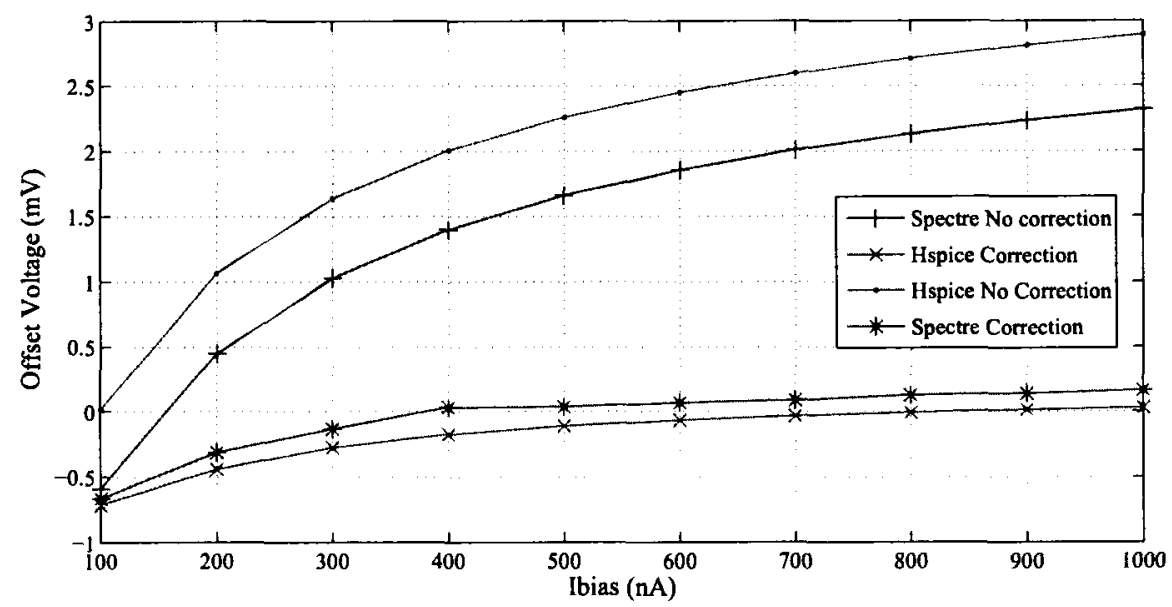

Figure 4.15: Offset voltages shown when the bias current is swept for a supply voltage of $0.6 \mathrm{~V}$ using HSPICE and Spectre models.

The offset and power dissipation were simulated for a $V_{D D}$ using $0.6 \mathrm{~V}$, while sweeping the bias current from $100 \mathrm{nA}$ to $1 \mu \mathrm{A}$ using HSPICE and Spectre. The results for power dissipation were almost identical with the largest difference being less than $10 \mathrm{nW}$ between the two simulators. The offset voltages are shown in Figure 4.15 with and without using the offset correction circuitry for HSPICE models and for Spectre models. The offset voltages follow the same trend with an increasing bias current, with slight variations in the actual offset values.

\subsection{Digital Successive Approximation Register and Control Logic}

\subsubsection{Custom Standard Cell Design}

A custom static CMOS library was designed to implement the logic. The logic was optimized for noise immunity and low power instead of high speed operation. The noise margins were maximized by ensuring that the PMOS and NMOS transistors 


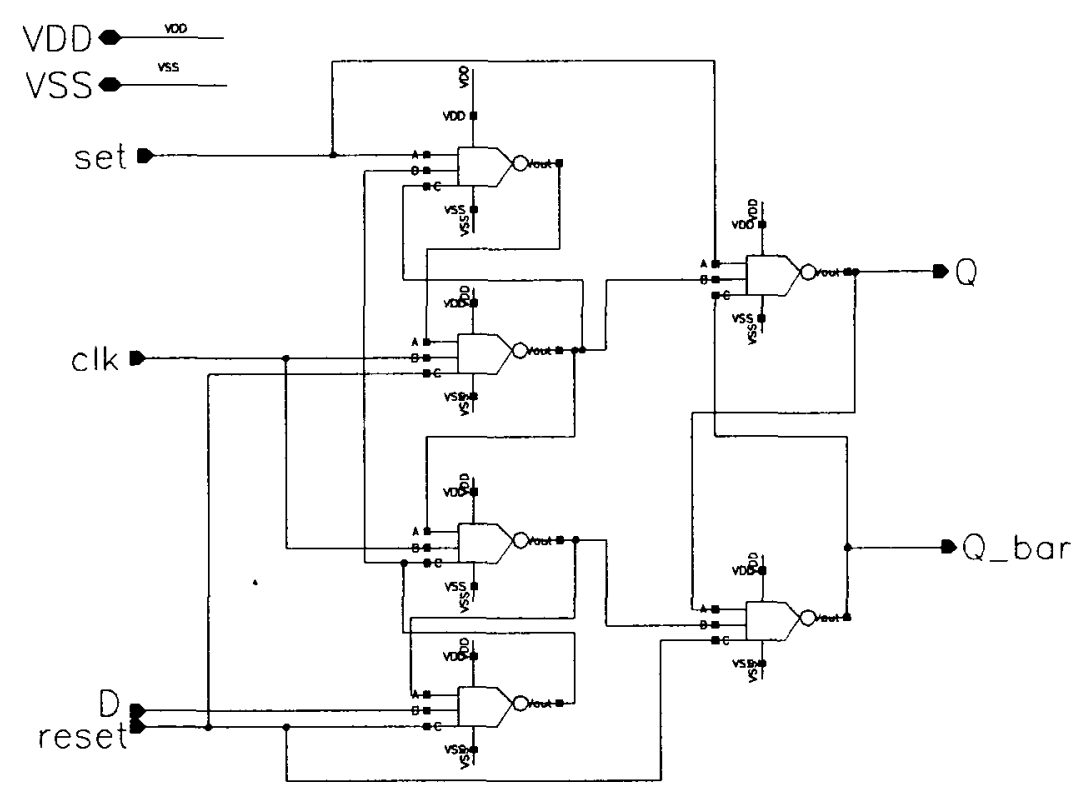

Figure 4.16: D-flip flop schematic used for SAR digital circuit.

had approximate equal drive strength and low power was achieved through making use of the smallest possible devices. This allows for lower input capacitance and thus less power dissipation. It is important to note that the digital logic makes a very small contribution to the total ADC power dissipation as seen in simulation and thus achieving the optimum logic to achieve minimum power dissipation was not a main concern. The digital circuit used to implement the SAR circuitry in [2] and [29] was based on [30]. The main building block of this circuitry is to have a D-flip flop that has an active-low reset and an active low set, with outputs $Q$ and its inverse. The flip flop architecture from [31] was selected which uses six 3-input NAND gates and the schematic is shown in Figure 4.16 


\subsubsection{Implementation of SAR and Control Logic}

The circuit to implement the successive approximation algorithm and provide all control signals for the ADC is shown in Figure 4.17. The core circuit is from [2] where it performs the successive approximation algorithm. The core circuit works as a shift register where the first flip flop is set to high either by a reset or from the previous conversion cycle, while the other flip flops are reset to low. The "1" is shifted through the flip-flops each clock cycle which turn on the lower flip-flops connected to compare bus from the comparator. If compare is high the next flip flop clocks the previous flip-flop and allows it to store the compare signal. Thus each bit is determined and stored depending on the signal from the comparator. The circuit is reset and a new conversion cycle begins. The full SAR schematic is shown in Figure 4.17. Extra logic was required to generate the control signals for the comparator and for the switch to reset the capacitor array which is showed in Figure 4.18. This required another reset circuit in order to generate these control signals once reset is initiated, otherwise the first ADC cycle would be incorrect. The reset circuitry would be important if the ADC was to be powered on and off often to save power.

\subsubsection{Layout and Simulation Results for SAR and Control Logic}

The layout of the SAR circuit is shown in Figure 4.19. The dimensions are $151 \mu \mathrm{m}$ by $180 \mu \mathrm{m}$ with the reset circuit located in the top left corner of Figure 4.19 with the majority of area used by the SAR core. The layout uses 4 metal layers and incorporates a custom logic cell design. The input and output signals are described 


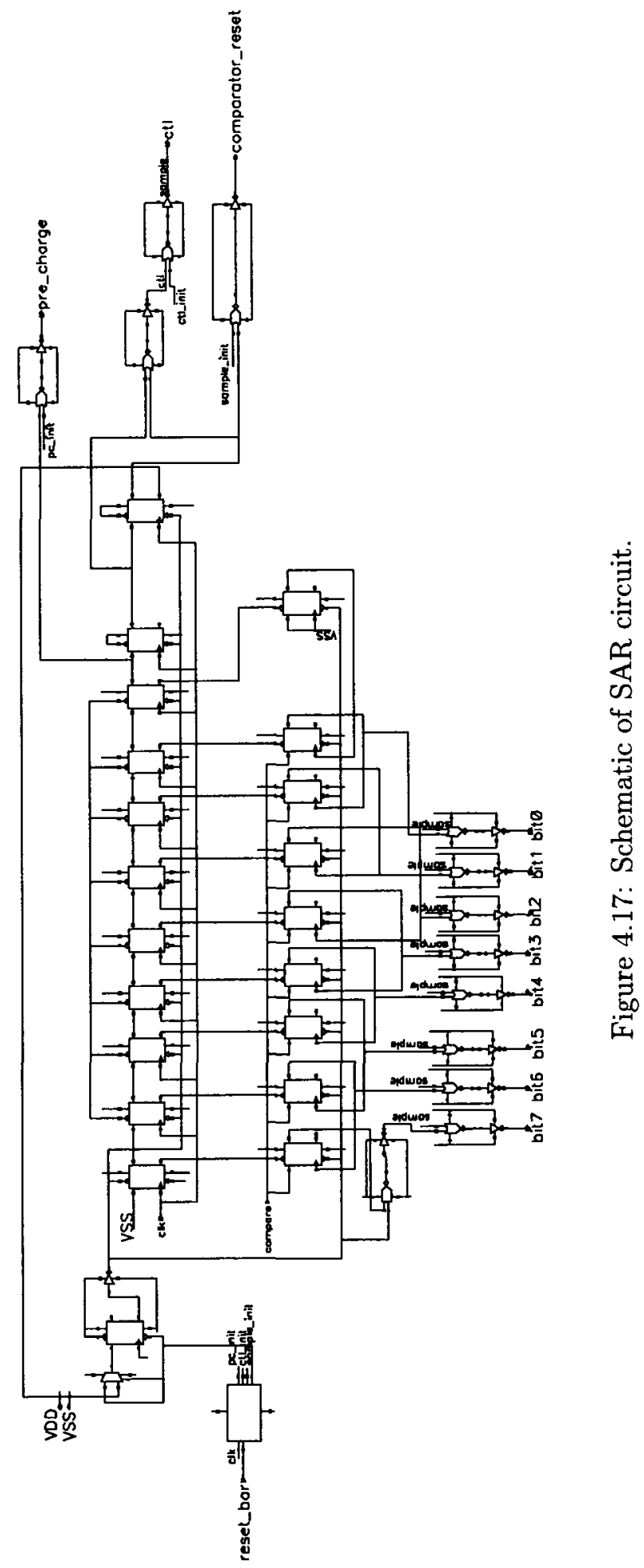




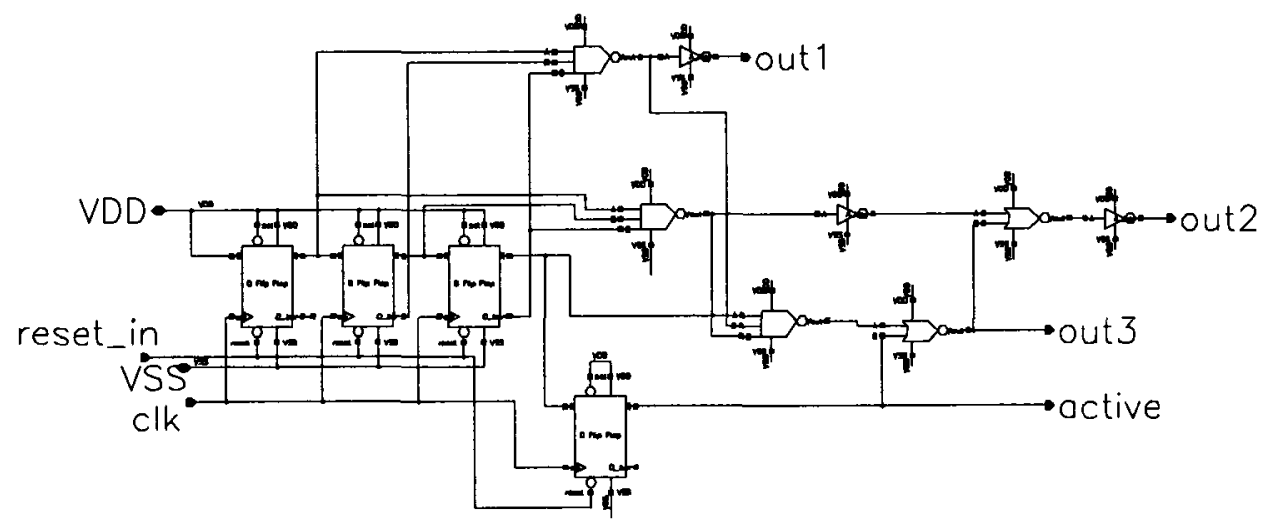

Figure 4.18: Schematic of circuit to initialize SAR circuit.

Table 4.4: Summary of signals for SAR circuit.

\begin{tabular}{|l|l|l|}
\hline Signal & Type & Description \\
\hline VDD & Power & Separate Digital Supply \\
\hline VSS & Power & OV for ground \\
\hline clk & Input & The clock signals used by flip-flops \\
\hline compare & Input & Output from the comparator \\
\hline reset_bar & Input & Resets flip flops, asynchronous low \\
\hline pre_charge & Output & $\begin{array}{l}\text { Resets top plate and used to pre- } \\
\text { charge comparator }\end{array}$ \\
\hline ctl & Output & $\begin{array}{l}\text { Resets top plate and uses by sam- } \\
\text { pling switch }\end{array}$ \\
\hline comparator_reset & Output & Used to reset comparator \\
\hline bit0..bit7 & 8-bit Output & Represents bit 0 to bit 7 of ADC \\
\hline
\end{tabular}




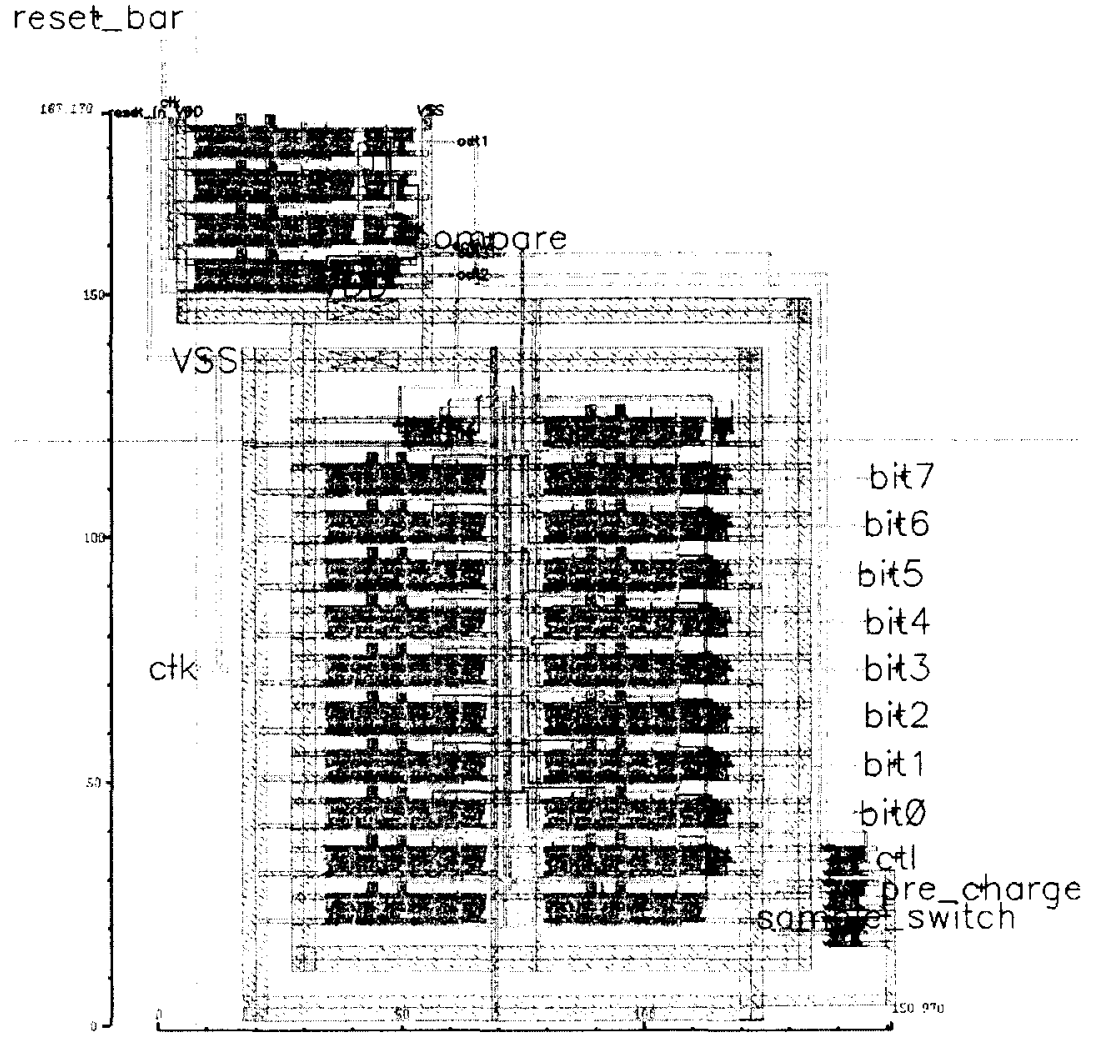

Figure 4.19: Layout of SAR circuit. 


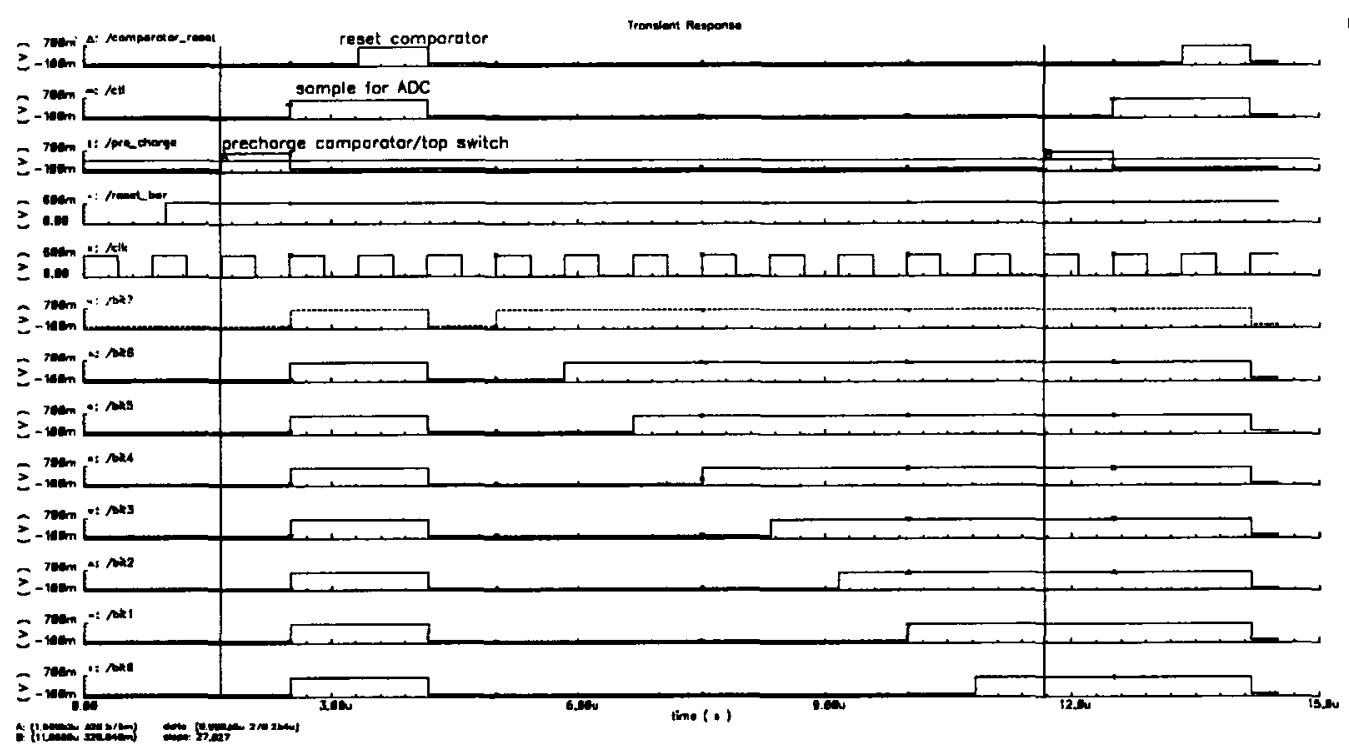

Figure 4.20: Input and output signals from SAR circuit.

in Table 4.4 and shown in Figure 4.20. As shown in Figure 4.20 the conversion cycle is as follows. First, precharge goes high, which will pre-charge the offset correction capacitor for the comparator and the capacitor for the top switch to reset the capacitor array. Next, ctl goes high, which will allow the top switch to reset the capacitor array to analog ground and at the same time sample the analog signal. Next, comparator_reset goes high which will allow the comparator to perform offset correction, while still sampling the analog signal. Next, all the binary weighted capacitors are connected to analog ground, and the top plates of the capacitor array gets set to analog GND $-V_{i n}$. The next 8 clock cycles the successive approximation algorithm is performed. This total conversion cycle is 12 clock cycles.

\section{Power Dissipation}

In Figure 4.21 the power dissipation with respect to supply voltage shows a quadratic relationship and in Figure 4.22 the power dissipation shows a linear relationship with 


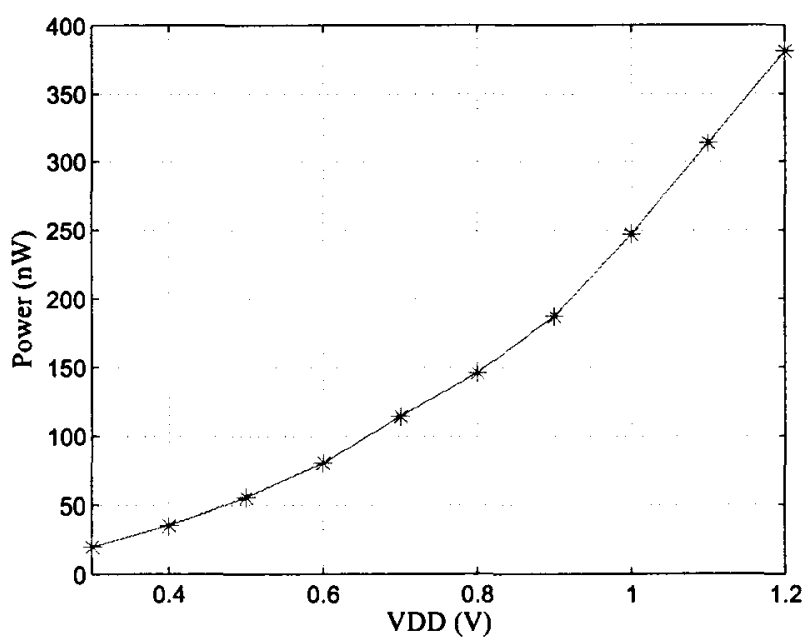

Figure 4.21: Total digital power dissipation for different supply voltages for clock of $1.2 \mathrm{MHz}, f_{S}=100 \mathrm{kS} / \mathrm{s}$.

frequency. This holds as for static CMOS digital logic power dissipation is given by [26],

$$
P=\frac{1}{2} V_{D D}^{2} C_{L} f+I_{l e a k} V_{D D}
$$

where $I_{l e a k}$ is the sub-threshold leakage that exists when $V_{G S}$ is approximately $0 \mathrm{~V}$ and from gate leakage. The total power dissipation of the digital circuitry is much lower than the comparator as seen Table 4.1. Also when process and mismatch variation as shown in Figure 4.23 are included for the digital circuitry when a $0.6 \mathrm{~V}$ supply and a $1.2 \mathrm{MHz}$ clock was used, the mean power was $82.7 \mathrm{nW}$ with a standard deviation of $5 \mathrm{nW}$. The total digital power dissipation varies slightly with process and mismatch variation and it is not a significant contributor to total power dissipation of the entire ADC and hence less design effort was placed on the digital components as opposed to the analog components for optimizing for low power dissipation. 


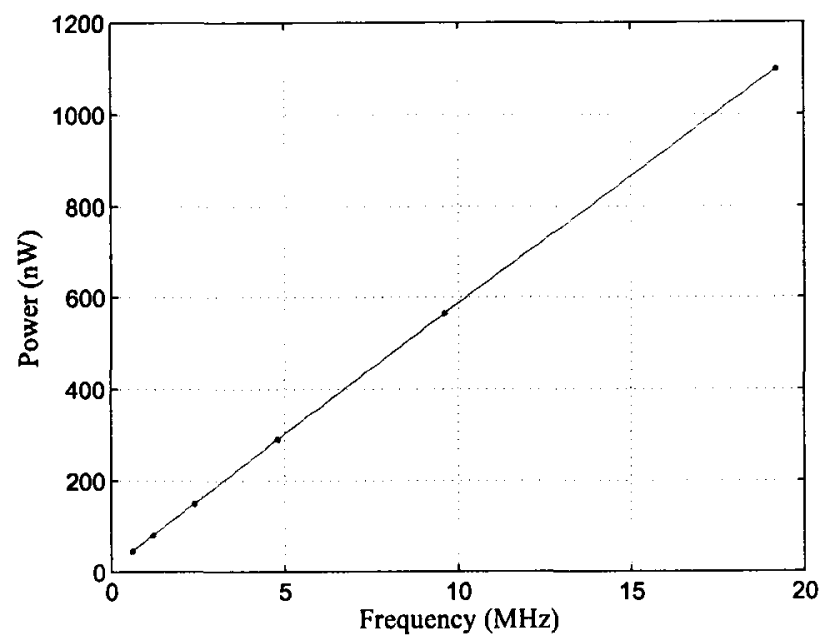

Figure 4.22: Total digital power dissipation for different frequencies for a supply of $0.6 \mathrm{~V}$.

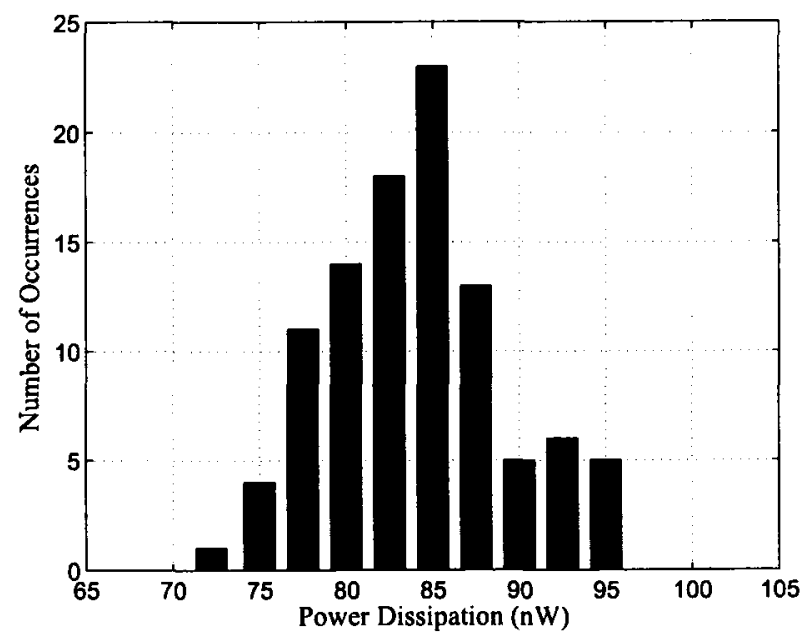

Figure 4.23: Monte Carlo Statistical Analysis simulation of 100 runs with process and mismatch variation with a supply of $0.6 \mathrm{~V}$ and a clock of $1.2 \mathrm{MHz}, f_{S}=100 \mathrm{kS} / \mathrm{s}$. 


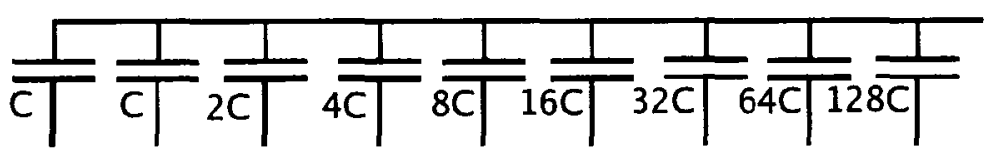

Figure 4.24: Binary weighted capacitor array schematic.

\subsection{Capacitor Array}

The capacitor array was initially implemented using the schematic shown in Figure 4.24 which was used in [29]. The capacitor array was to have the LSB capacitor equal to $50 \mathrm{fF}$, which was to be implemented using four $200 \mathrm{fF}$ capacitors in series. This meant the MSB capacitor was $6.4 \mathrm{pF}$, implemented using 32 parallel capacitors of $200 \mathrm{fF}$. Antenna violations did not make this implementation feasible. A large antenna diode was required at the top plate of the capacitor array. This posed two problems: the first being that the antenna diode could be forward biased as the top plate of the capacitor array reaches negative voltages during the conversion cycle, which could lead to charge loss and errors at the output. The second problem was that it added a large amount of parasitic capacitance at the top plate of the capacitor array. Antenna violations also prevented the use of series MIMcaps, which further made this implementation for the capacitor array more difficult. The solution was to use an attenuation capacitor array to be described next.

\subsubsection{Attenuation Capacitor Array}

The capacitor array was implemented using an attenuation capacitor as shown in Figure 4.25. An attenuation capacitor array was selected which allowed for smaller 


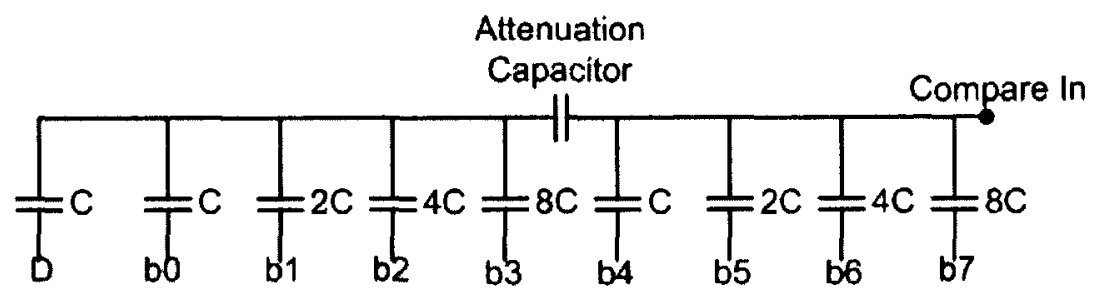

Figure 4.25: Binary weighted capacitor array schematic using attenuation capacitor. capacitors to be used. This capacitor array design was implemented for an 8-bit SAR $\mathrm{ADC}$ in $[13,14,32]$. The attenuation capacitor given by [17] is:,

$$
\mathrm{C}_{\mathrm{att}}=\frac{(\text { Sum LSB Capacitors })}{(\text { Sum MSB Capacitors })} \mathrm{C}
$$

where the sum of LSB capacitors is 16, the sum of MSB capacitors is 15, and C is $211 \mathrm{fF}$. The capacitor array using an attenuation capacitor allows for lower power and smaller area than the conventional capacitor array shown in Figure 4.24. One trade-off for the attenuation capacitor implementation is that it can degrade the INL and DNL of the ADC if the attenuation capacitor is not the ideal value especially around the middle of the input range [14]. Using the above equation for the attenuation capacitor this results in an attenuation capacitor of $225 \mathrm{fF}$, a MSB capacitor of $1.69 \mathrm{pF}$, and the LSB capacitor of $13.2 \mathrm{fF}$. Post-layout simulations with take into account extra parasitic capacitance determined that the attenuation capacitor should be approximately $235 \mathrm{fF}$. This attenuation capacitor needs to have an accurate value and with process variation during fabrication it will likely be different than the expected capacitance. 


\subsubsection{Tunable Capacitor}

One technique to mitigate the effects of process variations on the attenuation capacitor is to make it tunable as shown in Figure 4.26. The attenuation capacitor must be constant even as the voltage on the capacitor array is constantly changing for both nodes. Also the requirement of an extra control voltage is not feasible, as this would require feedback and control circuitry which would further increase power and complexity of the ADC. These factors make a varactor or MOScap type tunable capacitor to not be an ideal choice for the tunable attenuation capacitor. A solution for the tunable attenuation capacitor is a digitally tuned capacitor as shown in Figure 4.27. A similar circuit is described in reference [33] but is much more complex with a large area and high transistor count and uses a quartz dielectric for the capacitance. The goal is to have a very simple device that adds little to the total area and complexity of the ADC. The circuit schematic is shown in Figure 4.27 and contains three digital inputs and three parallel sets of capacitors, each with three series capacitors. Each unit capacitor, $\mathrm{C}$, is $175 \mathrm{fF}$. The middle series capacitors are $175 \mathrm{fF}, 350 \mathrm{fF}$ and $525 \mathrm{fF}$. A transmission gate switch is used to turn on or off each of these middle series capacitors. The value of the attenuation capacitor is given by,

$$
C_{\text {tune }}=\left(\frac{2}{C}+\frac{C_{1}}{C}\right)^{-1}+\left(\frac{2}{C}+\frac{C_{2}}{2 C}\right)^{-1}+\left(\frac{2}{C}+\frac{C_{3}}{3 C}\right)^{-1}
$$

where $\mathrm{C}$ is the unit capacitance, and $C_{1}, C_{2}$ and $C_{3}$ are binary values of 1 or 0.

After parasitic capacitors are added, post layout simulations indicated the tuning range to be between $231 \mathrm{fF}$ and $287 \mathrm{fF}$, with eight separate capacitor values between these two extreme values as shown in Figure 4.28. The ideal attenuation capacitor 


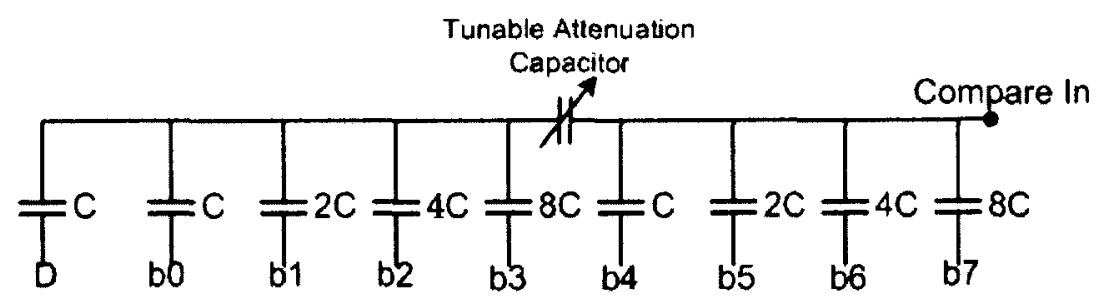

Figure 4.26: Binary weighted capacitor array schematic using tunable attenuation capacitor.

value for the tunable capacitor is $260 \mathrm{fF}$ which is in the middle of the two extremes. As shown in Figure 4.28 the post layout simulated capacitance is 30fF higher than using the equation which assumes ideal switches. The minimum capacitance range after two hundred Monte Carlo simulations is $210 \mathrm{fF}$ to $260 \mathrm{fF}$ and the maximum capacitance range is $250 \mathrm{fF}$ to $318 \mathrm{fF}$. Even using these extreme Monte Carlo cases the $260 \mathrm{fF}$ is still achievable. Also shown in Figure 4.28 is one standard deviation above and below the mean capacitance which is about 9fF. During fabrication, the capacitor values will change due to process variations however there should still be eight distinct capacitor values, and the one that offers the best performance is selected during operation.

\subsubsection{Layout of Capacitor Array}

The linearity of the ADC is influenced by the mismatch of the capacitor array [10]. Thus it is important to ensure the capacitors are well matched as the INL and the DNL of the ADC can degrade due to capacitor mismatch. The maximum DNL due to capacitor mismatch is given in [17] by,

$$
\mathrm{DNL}_{\text {max }}=\left(2^{N}-1\right)|\Delta C|_{\text {max }}
$$




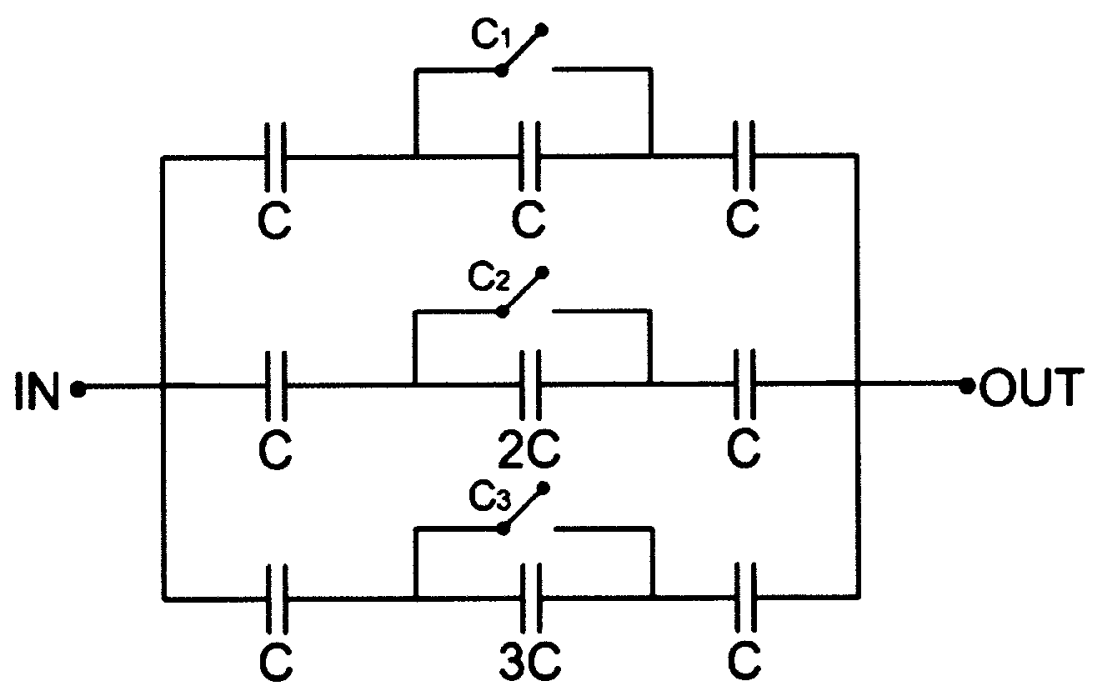

Figure 4.27: Schematic of tunable attenuation capacitor.

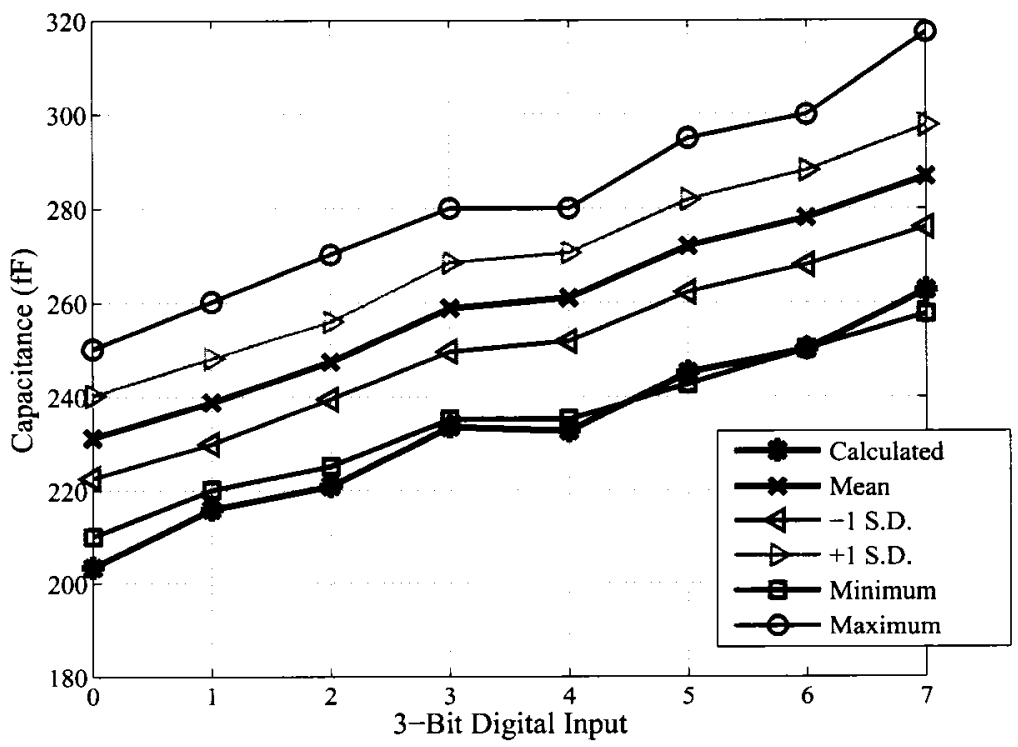

Figure 4.28: Monte Carlo Statistical Analysis simulation of 200 runs with process and mismatch variation for the tunable capacitor. 
where $\Delta C$ is the maximum capacitance mismatch and $\mathrm{N}$ is the number of bits. The maximum INL due to capacitor mismatch is given in [17] by,

$$
\mathrm{INL}_{\max }=\left(2^{N-1}\right)|\Delta C|_{\max }
$$

where $\Delta C$ is maximum capacitance mismatch and $N$ is the number of bits.

Some techniques to improve matching were: to use identical square geometries for the individual capacitor units; have a dummy capacitor ring to remove etching effects to the outside capacitors; and placing the capacitors in a common centroid orientation. The layout of the attenuation capacitor array with its schematic shown in Figure 4.25 is shown in Figure $4.29 \mathrm{a}$. The attenuation capacitor is placed at the center of the layout with the other bit capacitors around this common reference point. The layout of the tunable capacitor array with its schematic in Figure 4.27 is shown in Figure $4.29 \mathrm{~b}$. This layout is the same as the layout shown in Figure 4.25 but the attenuation capacitor is replaced by the tunable capacitor. The tunable capacitor increased the total area of the capacitor array by 20 percent.

To minimize parasitic capacitance the top plates of the capacitor array were connected to the top plate of the MIMcaps, the interconnects were routed with higher metal layers and the smaller capacitors were placed closer to their respective switches in order to reduce their interconnect capacitance. Underneath the dummy capacitor ring, guard rings were placed around the entire capacitor array to improve isolation from the switching components of the ADC, such as the digital logic. 


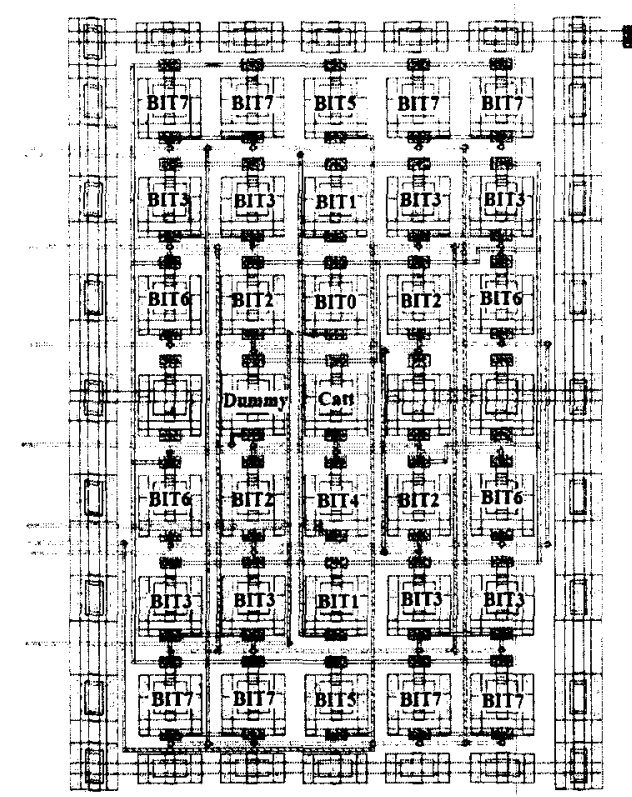

(a)

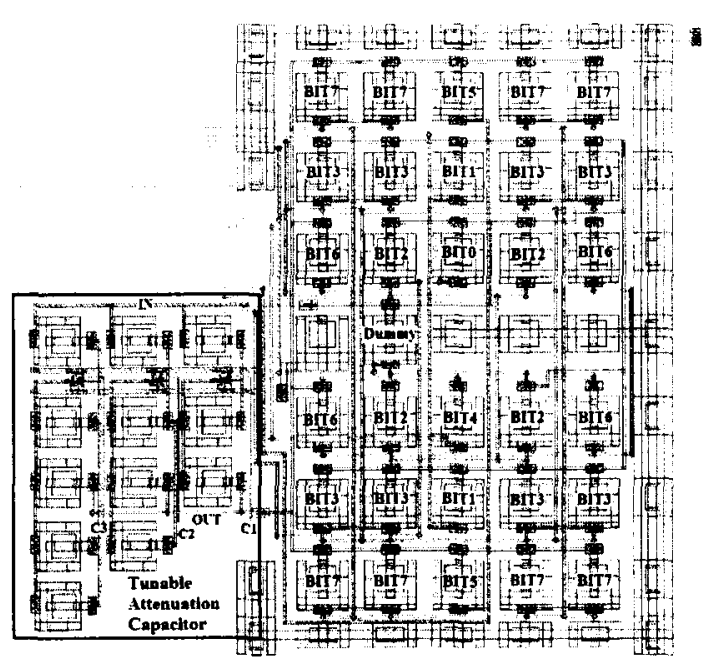

(b)

Figure 4.29: (a) Layout of attenuation capacitor array with dimensions $165 \mu \mathrm{m}$ by $240 \mu \mathrm{m}$. (b) Layout of tunable attenuation capacitor array with dimensions $165 \mu \mathrm{m}$ by $240 \mu \mathrm{m}$ with additional tunable capacitor of $80 \mu \mathrm{m}$ by $120 \mu \mathrm{m}$. 


\subsection{Analog Switches, Switch Array and Output Buffers}

There are three different switches that need to be designed. One switch is used to reset the top plate of the capacitor array to analog GND, where two switches are required (one on each side of attenuation capacitor). A network of nine switches is required that drive each capacitor bit and the dummy capacitor. A large switch is required to select between analog GND and $V_{R E F}$.

\subsubsection{Switch to Reset Capacitor Array}

The first ADC was intended to have a full input range as $V_{D D}$ is already low and decreasing the input range would cause the LSB voltage to be smaller. A switch is required to reset the top plate of the capacitor array to GND during sampling, and for the switch to remain off as the voltage on the capacitor array ranges from $-V_{\text {ref }}$ to $V_{\text {ref }} / 2$ during the approximation phase. A full input range means that the ADC will have negative voltages and this poses two problems: 1) An NFET will become forward biased with the presence of negative voltages causing significant leakage current and thus charge loss on the capacitor array; and, 2) A PFET fixes the leakage problem but it can cannot fully reach GND. The circuit taken from [29] allows for a full input range, and its schematic is shown in Figure 4.30. First, Precharge goes high, while Control is held low. This turns on transistor M1 and applies $G N D+V_{T P}$ to the top plate of the capacitor while the bottom plate of the capacitor has $V_{D D}$ applied to it. Next, Precharge goes low, while Control goes high. With transistor M1 off, GND is then applied to the bottom plate of the capacitor. This forces the voltage at the top 
plate of the capacitor array to become close to negative $V_{D D}$. This allows transistor M3 to remain ON allowing the top plate of the capacitor array to be grounded during the sampling phase of the analog signal. The control signals for this switch are shown in Figure 4.31 when a supply of $0.6 \mathrm{~V}$ is used. When the ADC is sampling, transistor M3 has a $V_{G S}=0.45 V$ which is enough to turn on the PFET transistor.

When the $\mathrm{ADC}$ is converting, the gate of $\mathrm{M} 3$ is charged to $V_{D D}$. During this phase, it is important for the capacitor array to not drain charge and by having the gate of M3 at $V_{D D}$, this allows transistor M3 to have a $V_{G S}<0 V$ as the maximum voltage on the capacitor array is $V_{D D} / 2$. With the drain of transistor $\mathrm{M} 3$ at $0 \mathrm{~V}$, this ensures transistor M3 is always operating deep in sub-threshold regime and allows for low leakage currents which is important when the sample rate is low to ensure that minimum charge is lost from the capacitor array.

The second ADC uses a transmission gate to reset the top plate of the capacitor array, as the input range is one half of the supply voltage allowing for all voltage levels to be between the power rails.

\subsubsection{Analog Switch Array}

All the switches were implemented using CMOS transmission gates as the signals passed are between $V_{S S}$ and $V_{D D}$. The schematic for the switch array for ADC, ICGCUADC is shown in Figure 4.32. It contains nine transmission gates (eight for each bit capacitor and one for the dummy capacitor) and a larger transmission gate to switch between Analog IN and $V_{R E F}$. All of the switch array transmission gates were sized to be $1 \mu \mathrm{m} / 0.12 \mu \mathrm{m}$ for the NFET and $2 \mu \mathrm{m} / 0.12 \mu \mathrm{m}$ for the PFET, except the 


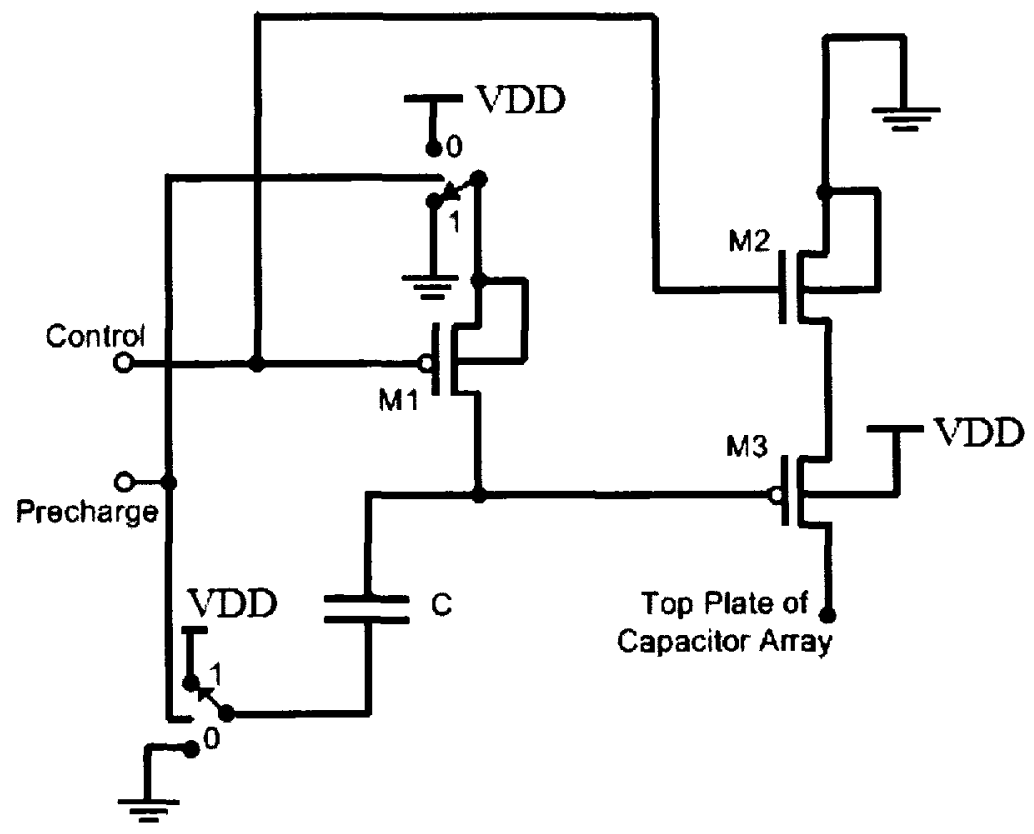

Figure 4.30: Switch to GND capacitor array for ICGCUADC. PFET M1 is $10 \mu \mathrm{m} / 0.12 \mu \mathrm{m}$, M2 is a high-threshold NFET $8 \mu \mathrm{m} / 0.24 \mu \mathrm{m}$, PFET M3 is $10 \mu \mathrm{m} / 0.12 \mu \mathrm{m}$ and capacitor $\mathrm{C}$ is a $1.09 \mathrm{pF}$ MIMcap.

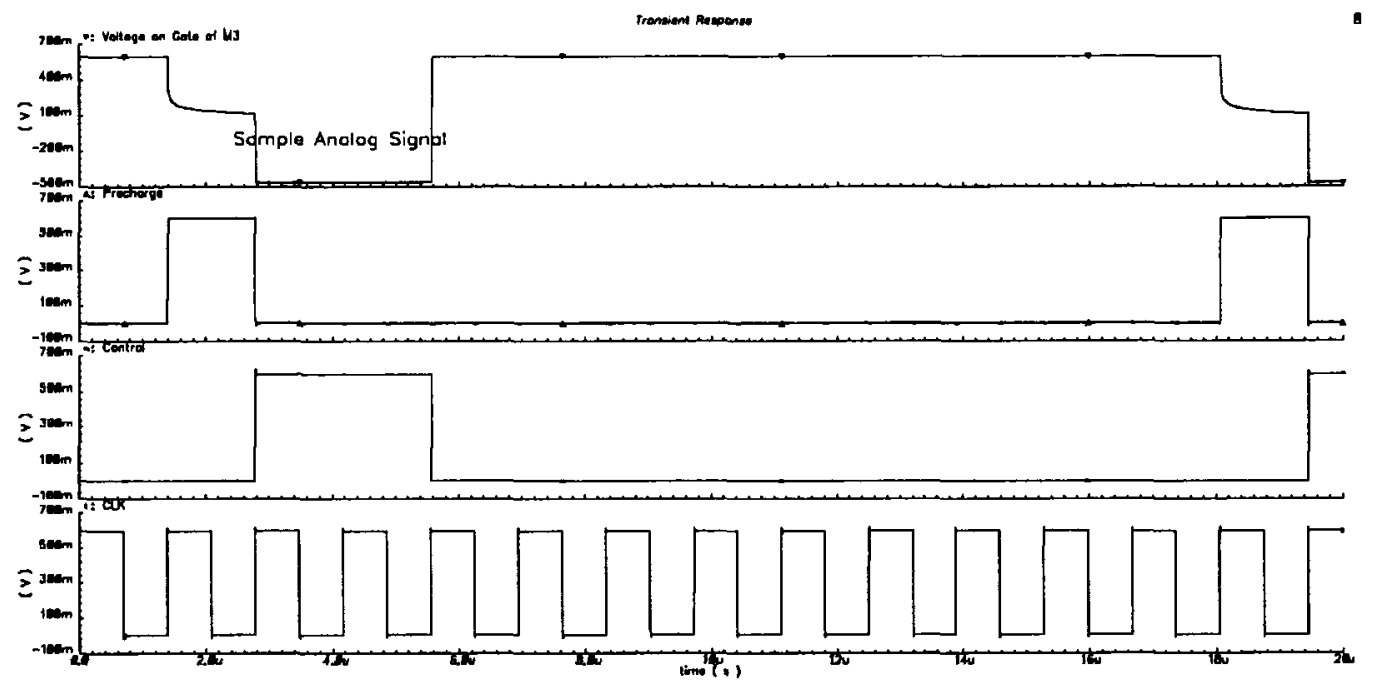

Figure 4.31: Control signals for switch to GND capacitor array $(V D D=0.6 V)$. 
largest capacitor on each side of the attenuation capacitor (bit 3 and bit 7) which are made $4 \mu \mathrm{m} / 0.12 \mu \mathrm{m}$ for the NFET and $8 \mu \mathrm{m} / 0.12 \mu \mathrm{m}$ for the PFET. Each transmission gate outputs $V_{S S}$ when the switch has its control signal set to 0 . When the control signal for each transmission gate is set to 1 , the transmission gate outputs Analog IN or $V_{R E F}$, depending on whether the ADC is sampling or converting the signal, respectively. The transmission gate that switches between Analog IN and $V_{R E F}$ is made the largest of the switches with an NFET size of $12.5 \mu \mathrm{m} / 0.12 \mu \mathrm{m}$ and a PFET size of $25 \mu \mathrm{m} / 0.12 \mu \mathrm{m}$, as all bit capacitors will have this switch's resistance in series with its own bit switch resistance and thus by increasing its size, it decreases its ON resistance.

The switch array for ADC ICGCUKAH is similar to the circuit shown in Figure 4.32. Modifications for the switch array for this ADC include: 1) Signal $V_{R E F}$ in Figure 4.32 becomes connected to $V_{D D}$ and 2) $V_{S S}$ in Figure 4.32 becomes connected to analog GND $\left(V_{D D} / 2\right)$. This will allow the input range to be adjusted from $0 \mathrm{~V}$ $V_{R E F}$ (for ICGCUADC) to $V_{D D} / 2-V_{D D}$ (for ICGCUKAH).

As mentioned in [10] the minimum supply voltage is approximated by,

$$
V D D_{\min } \geq V_{T N}+V_{T P}
$$

For low supply voltage the switches have a very large resistance as they are operating in sub-threshold and this can limit the bandwidth of the ADC which is shown in Figure 4.33 where for low supply voltages the $O N$ resistance is much higher and exponentially decreases as the supply voltage is increased. From [20] the settling 


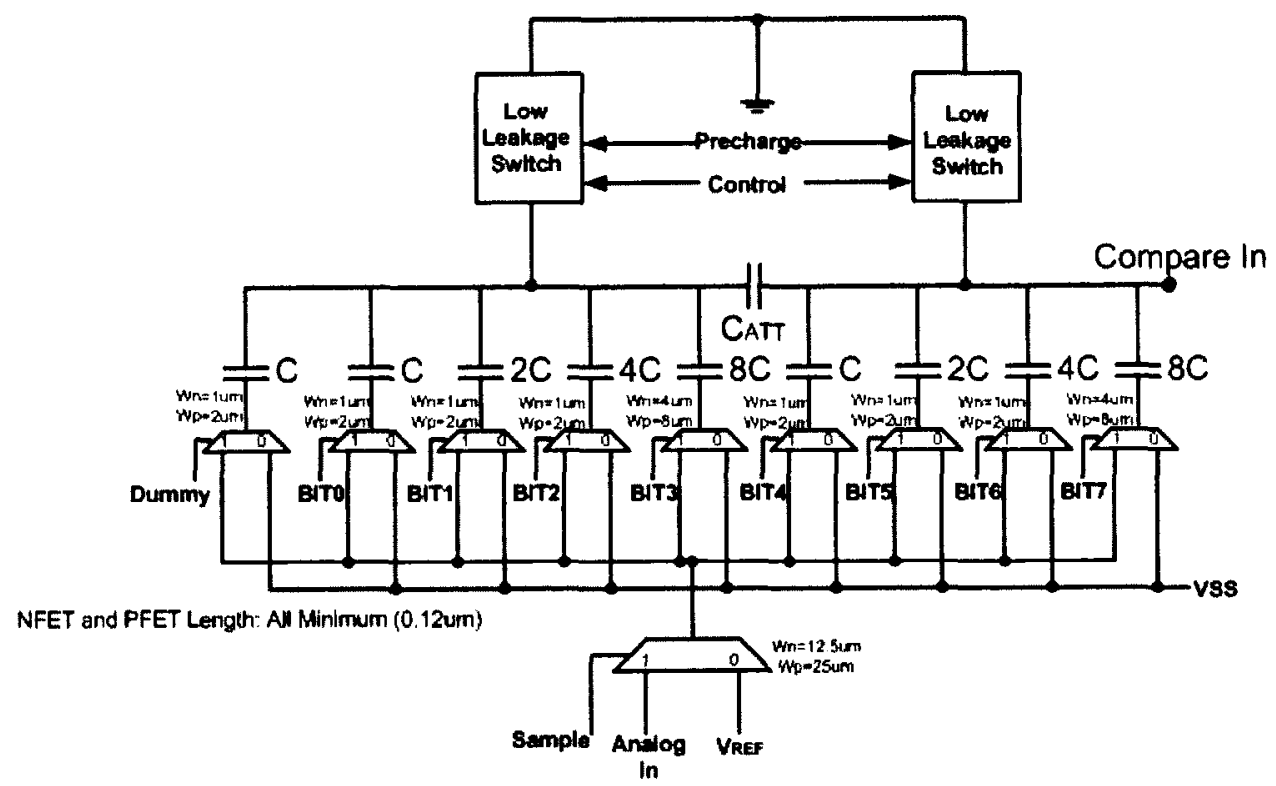

Figure 4.32: Schematic of switch array for ADC, ICGCUADC.

error for 0.5-LSB accuracy is given by,

$$
e^{\frac{-T}{R C}}<\frac{1}{2^{(N+1)}}
$$

And thus, for 9-bit accuracy (8-bit is required) the time constant should be $7 R_{O N} C$. The analog switches and their maximum ON resistances are shown in Table 4.5 when the supply is $V_{D D}=0.4 \mathrm{~V}$. From this table the charge time for worst case resistance for a 9-bit $\mathrm{ADC}$ is $0.898 \mu \mathrm{s}$. Taking into account the 12 clock cycles for each conversion cycle, this results in a maximum ideal sample rate of $90 \mathrm{kS} / \mathrm{s}$ for charging the individual bit capacitors.

Other factors such as the time required to reset the capacitor array to analog GND through the switches on the top plate and the bandwidth of the comparator will further degrade the maximum sample rate of the ADC. 


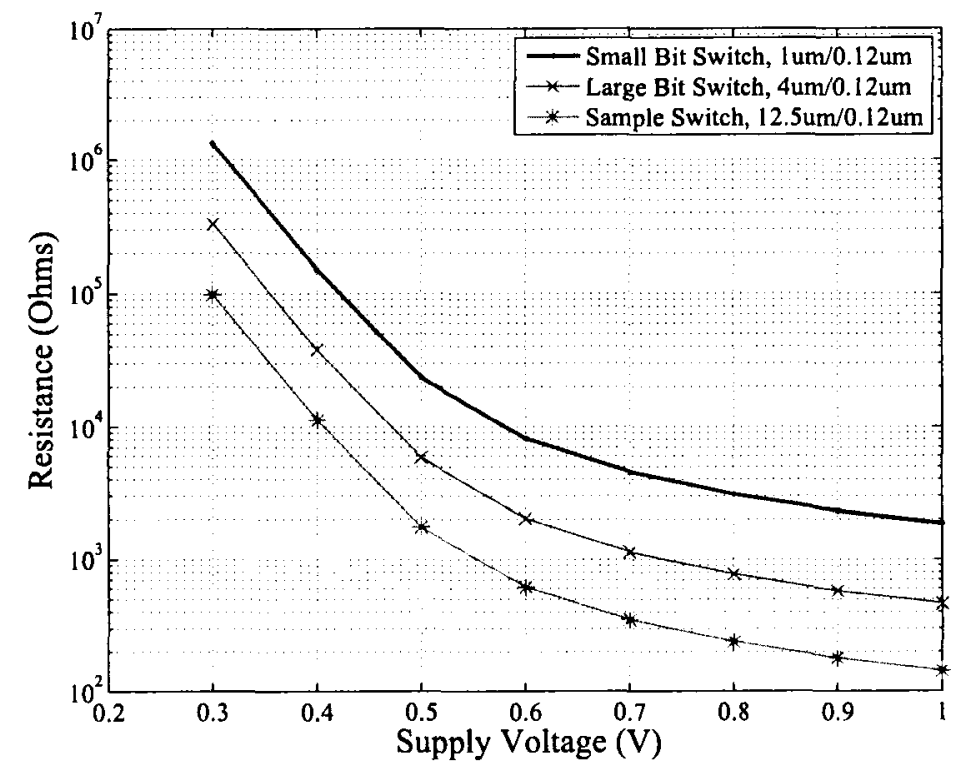

Figure 4.33: The maximum ON resistances used for the analog switches for different supply voltages.

Table 4.5: Post layout simulation results of $O N$ resistances for the analog switches and their respective settling times for each bit capacitor $V_{D D}=0.4 \mathrm{~V}$.

\begin{tabular}{|l|l|l|l|}
\hline NMOS Size & $\begin{array}{l}\text { Maximum } R_{O N} \\
(\text { Ohms) }\end{array}$ & $\begin{array}{l}\text { Capacitor } \\
\text { Value (fF) }\end{array}$ & $\begin{array}{l}\text { Settling } \\
(\mathrm{sec})\end{array}$ \\
\hline $1 \mu \mathrm{m} / 0.12 \mu \mathrm{m}$ & $152 \mathrm{k}$ & 211 & $0.2245 \mu$ \\
\hline $1 \mu \mathrm{m} / 0.12 \mu \mathrm{m}$ & $152 \mathrm{k}$ & 422 & $0.449 \mu$ \\
\hline $1 \mu \mathrm{m} / 0.12 \mu \mathrm{m}$ & $152 \mathrm{k}$ & 844 & $0.898 \mu$ \\
\hline $4 \mu \mathrm{m} / 0.12 \mu \mathrm{m}$ & $37.9 \mathrm{k}$ & 1688 & $0.4478 \mu$ \\
\hline
\end{tabular}




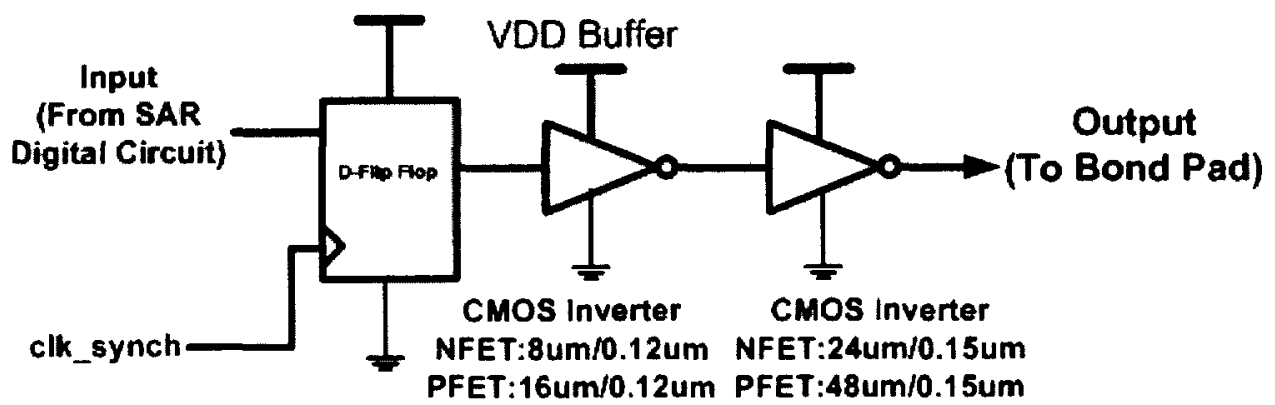

Figure 4.34: Schematic of buffer circuit to drive each bit. One buffer circuit is required per bit.

\subsubsection{Output Buffers}

The SAR digital logic outputs the successive approximation algorithm where the bits all pulse high for one clock cycle during the sampling phase and then each bit goes high and stays high for the rest of the conversion cycle or goes low the next clock cycle depending if its a "1" or " 0 " respectively. To simplify testing, these outputs were converted to simple bits of 1's and 0's to be easily read by a logic analyzer. This was achieved by using a signal that goes high after the conversion cycle is complete to clock flip flops that will store the ADC output and then output them to a 2-stage CMOS buffer, where the schematic of this circuit is shown in Figure 4.34. The signals before and after the flip flop and buffer is shown in Figure 4.35 where the outputs become 1's and 0's that only represent the ADC output bits. 


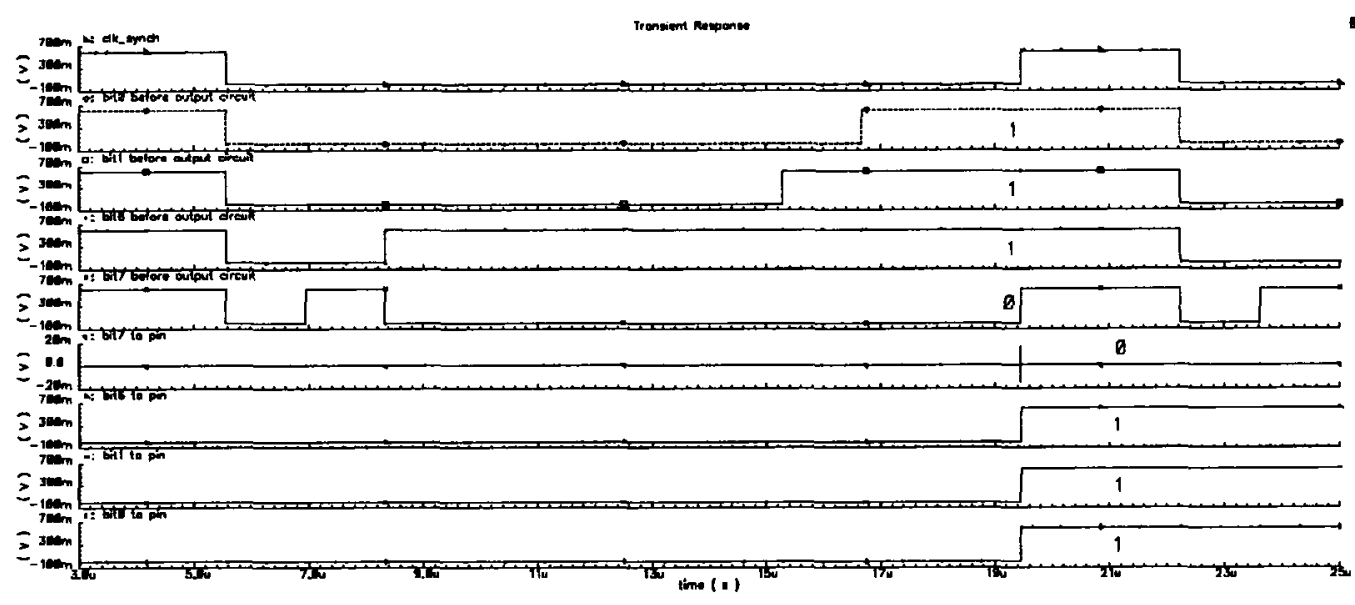

Figure 4.35: Signals before and after the output circuit.

\subsection{Analog to Digital Converter Cores}

\subsubsection{Analog to Digital Converter 1: 8-bit ADC with Full Input Range}

The first 8-bit ADC, ICGCUADC, uses an analog ground of zero volts. This allows the $\mathrm{ADC}$ to receive inputs between 0 and $V_{D D}$. The advantages of using a full input range are that it increases the size of the LSB voltage thus relaxing the accuracy requirements of the comparator and it can remove extra circuitry to generate the $V_{R E F}$ voltage since the supply voltage can be used. This was achieved by making use of the low leakage switch shown in Figure 4.30. Such a switch is required as the voltage on the top plate of the capacitor array can swing between $-V_{D D}$ and $V_{D D} / 2$. To achieve better performance at an analog ground of $0 \mathrm{~V}$, zero-threshold input transistors were used for the comparator. The negative voltages at the top plate of the capacitor array prevented the use of the tunable attenuation capacitor, as the parasitic diodes in the source and drain for the transistors in the tunable capacitor would become forward biased leading to leakage of the capacitor array and 
the possibility of latch-up.

The core layout of the first ADC chip is shown in Figure 4.36. The component using the largest layout area is the the capacitor array followed by the SAR and control digital circuitry, the buffers, the top plate switches, the comparator and then the switch array. The dimensions of the core layout as shown on Figure 4.36 are $475 \mu \mathrm{m}$ by $390 \mu \mathrm{m}$. The SAR and buffer circuitry were placed far away from the sensitive analog comparator and capacitor array. This is important as the switching from the digital CMOS logic generates substrate noise.

Figure 4.37 shows the first ADC working when the input is the maximum allowed input $\left(V_{R E F}\right)$. First, the switch to reset the top plate is pre-charged to slightly above $-V_{D D}$. At the same time, the sample control signal is high, which samples the analog signal, $V_{I N}$. As the analog signal is being sampled, the top plate of the capacitor array is discharged to $0 \mathrm{~V}$. Next, the switch to reset the top plate is open circuited while the bottom plates of each bit capacitor are set to ground. This forces the top plate of the capacitor array to reach $-V_{I N}$. Next, the conversion cycle begins, where $V_{R E F} / 2$ is added, then $V_{R E F} / 4$, then $V_{R E F} / 8$ and repeated until the last bit is found. Each time the top plate of capacitor array is negative, this represents a "1" for the $\mathrm{ADC}$ and each time it is a positive, this represents a " 0 " for the ADC. During the entire conversion cycle the top plate is negative, therefore the output was 11111111 , which was stored by the digital SAR logic. 


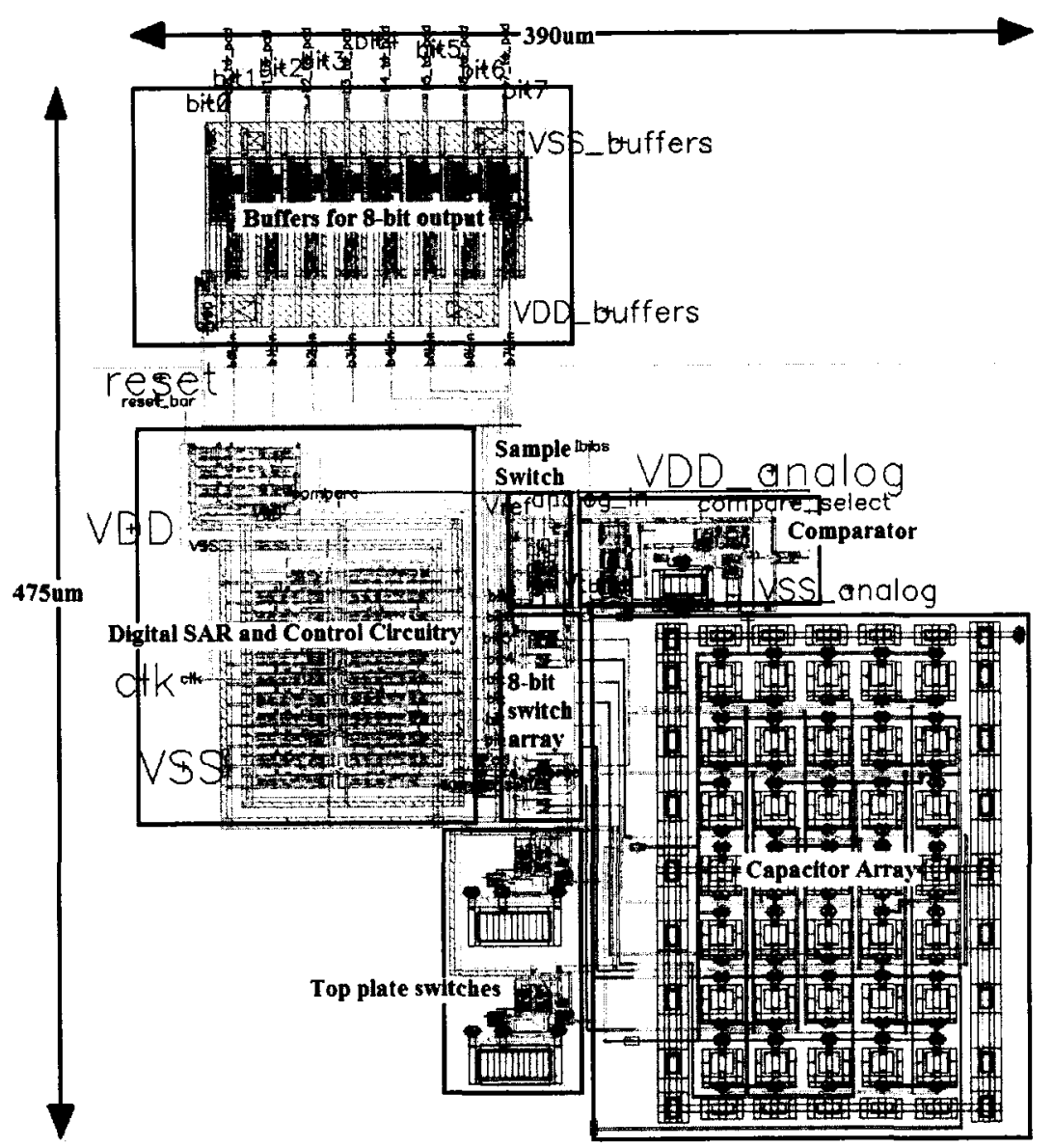

Figure 4.36: The layout of ADC core ICGCUADC. The dimensions are $475 \mu \mathrm{m}$ by $390 \mu \mathrm{m}$. 


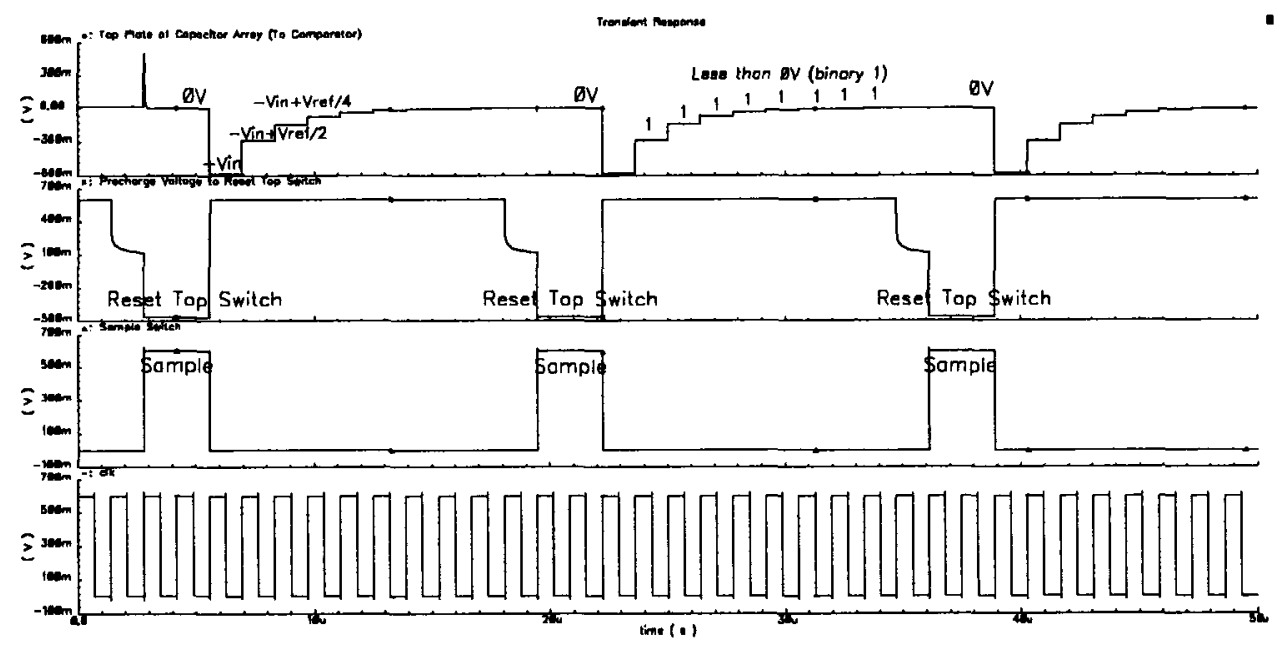

Figure 4.37: The signals for the voltage of the top plate of the capacitor array, voltage for pre-charging reset switch, sample signal and clk when the input is maximum $\left(V_{R E F}\right)$ for ADC ICGCUADC, with $V_{D D}=0.6 \mathrm{~V}$. Digital Output is 11111111.

\subsubsection{Analog to Digital Converter 2: 8-bit ADC with Tun- able Capacitor Array}

The second 8-bit ADC, ICGCUKAH, uses an analog ground of $V_{D D} / 2$. This allows the ADC to receive inputs between $V_{D D} / 2$ and $V_{D D}$. This means the voltage at the top plate of the capacitor array will be between $0 \mathrm{~V}$ and $0.75 V_{D D}$, with no negative voltages present. The LSB voltage is halved and thus the ADC becomes more sensitive to comparator offset, noise, capacitor mismatch, and temperature. The advantage of using this technique is that the top reset switch can be a transmission gate. This reduces the complexity of the $\mathrm{ADC}$ and also the power dissipation as it does not have to charge and discharge the large capacitor in the low leakage switch shown in Figure 4.30. The comparator is set at $V_{D D} / 2$ which allows the comparator to have NFET input transistors, and removes the addition of zero-threshold transistors.

The negative voltages at the top plate of the capacitor array were removed allowing 
the attenuation capacitor to be implemented using the tunable capacitor shown in Figure 4.27. The accuracy of the ADC needs to be improved as the LSB is halved compared to the case of the first $\mathrm{ADC}$, but the tunable attenuation capacitor should help achieve a more accurate attenuation capacitance. The second ADC is similar to the first $\mathrm{ADC}$ except for NMOS input transistors for the comparator, transmission gates to reset the top plate of the capacitor array, a tunable attenuation capacitor array and the input range is half that of the first ADC, ICGCUADC.

The core layout of the second ADC chip is shown in Figure 4.38. The component using the largest layout area again is the capacitor array followed by the SAR and control digital circuitry. The top plate switches are much smaller for the second ADC chip, but the tunable capacitor increases the size of the capacitor array. The dimensions of the core layout as shown on Figure 4.38 are $475 \mu \mathrm{m}$ by $390 \mu \mathrm{m}$, which is the same as the first ADC.

The Figure 4.39 shows the second $\mathrm{ADC}$ working when the input is the maximum allowed input $(2 \times$ analog GND). First, the sample control signal is high, which samples the analog signal, $V_{I N}$, while grounding the top plate through a transmission gate. As the analog signal is being sampled, the top plate of the capacitor array is discharged to analog ground $(0.3 \mathrm{~V})$. Next, the switch to reset the top plate is open circuited while the bottom plates of each bit capacitor are set to ground. This forces the top plate of the capacitor array to reach $2 \times$ analog GND $-V_{I N}$, which is approximately $0 \mathrm{~V}$ for this case. Next, the conversion cycle begins, where $V_{R E F} / 2$ is added, then $V_{R E F} / 4$, then $V_{R E F} / 8$ and repeated until the last bit is found. Each time the top plate of capacitor array is below analog ground $(0.3 \mathrm{~V})$, this represents a 1 for the ADC and each time it 


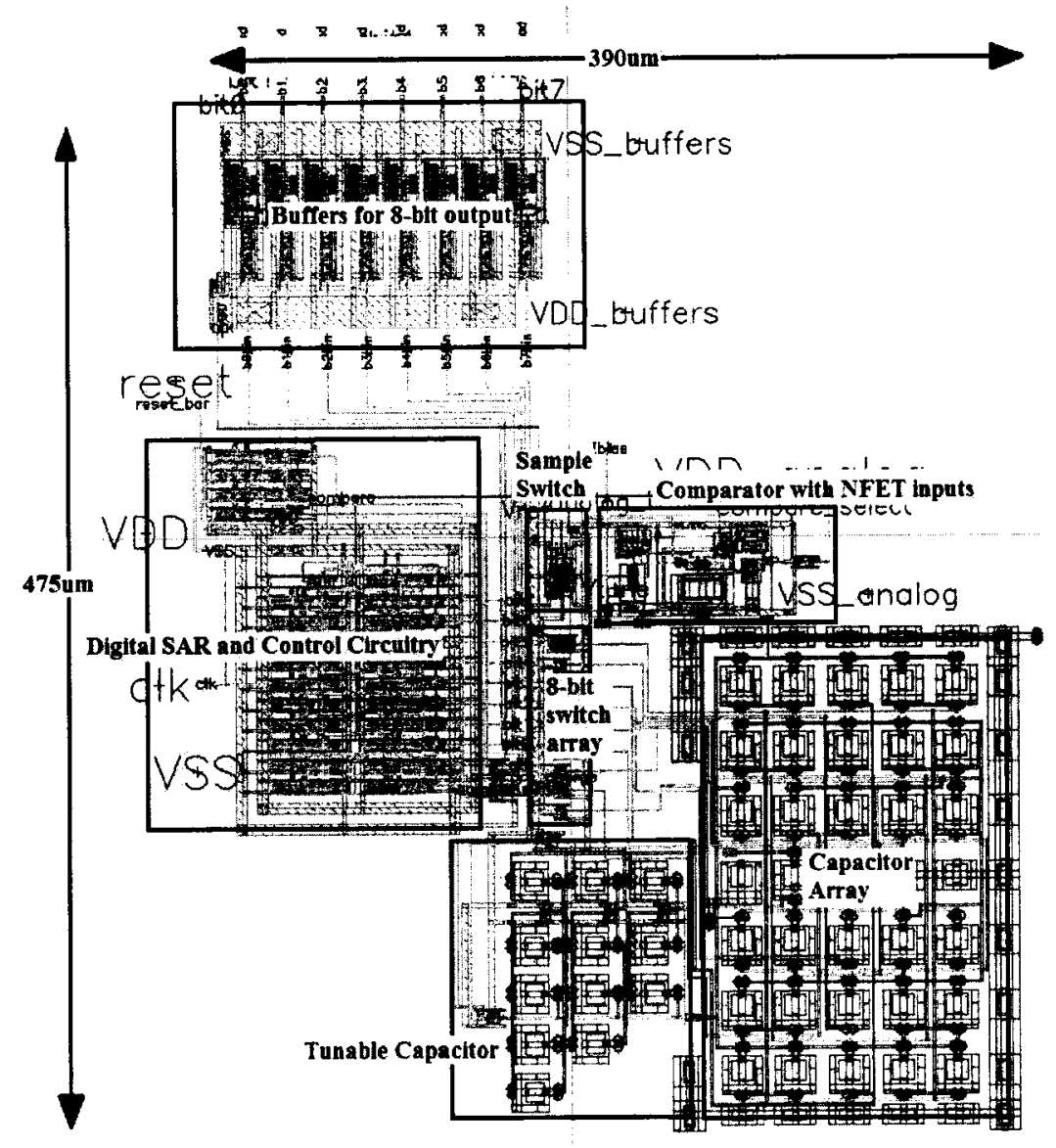

Figure 4.38: The layout of ADC core of ICGCUKAH. The dimensions are $475 \mu \mathrm{m}$ by $390 \mu \mathrm{m}$. 


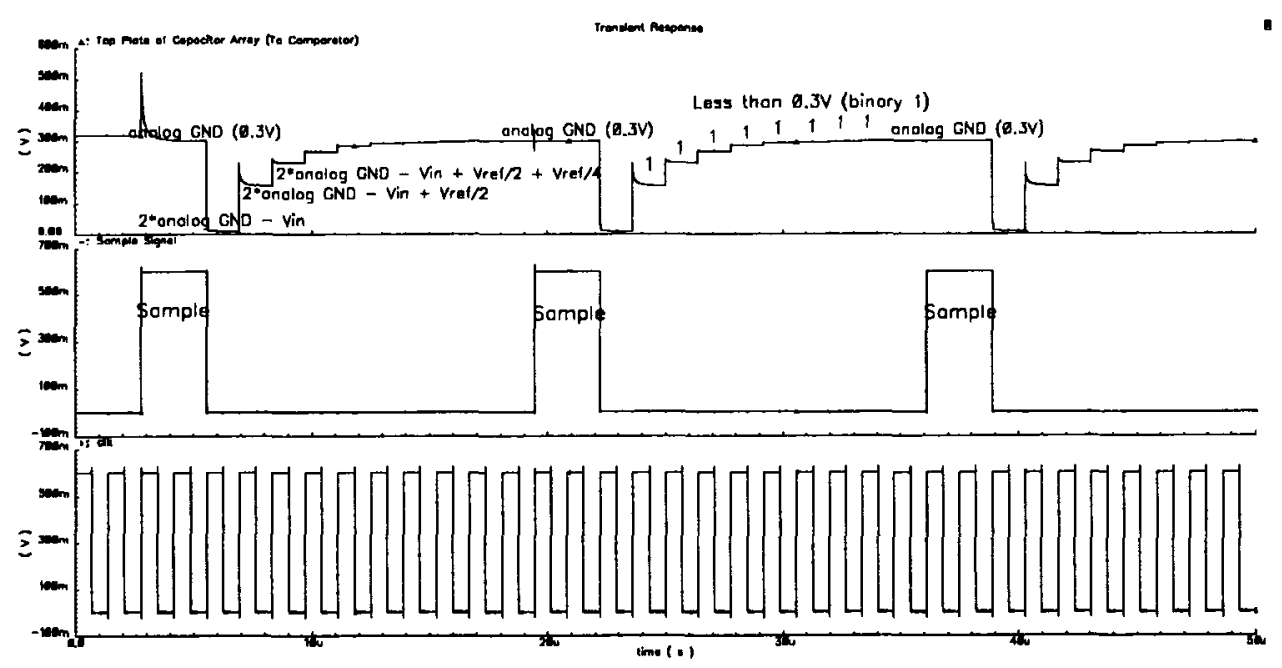

Figure 4.39: The signals for the voltage of the top plate of the capacitor array, sample signal and clk when the input is maximum ( $2 \times$ analog GND) for ADC 2 , with $V_{D D}=0.6 \mathrm{~V}$. Digital Output is 11111111 .

is above analog ground this represents a 0 for the ADC. During the entire conversion cycle the top plate is below $0.3 \mathrm{~V}$, therefore the output was 11111111 , which was stored by the digital SAR logic.

\subsection{Final Analog to Digital Layouts}

\subsubsection{Chip 1: ICGCUADC}

The first ADC chip, entitled ICGCUADC, is $1.25 \mathrm{~mm}$ by $1.0 \mathrm{~mm}$ and its final layout is shown in Figure 4.40. It includes the ADC core of Figure 4.36, with additional bond pads, a separate comparator to be tested individually from the ADC, metal fill, clock buffers, ESD protection (resistors and diodes), decoupling capacitors and guard rings to isolate the analog components from the rest of the circuitry. All the pins of Figure 4.40 are described in detail in Table 4.6. 
Table 4.6: Summary of ICGCUADC Pins.

\begin{tabular}{|c|c|}
\hline Name & Description \\
\hline BIT0 & Output of bit0 (digital, $0 \mathrm{~V}$ or $V_{D D}$ ) \\
\hline BIT1 & Output of bit1 (digital, $0 \mathrm{~V}$ or $V_{D D}$ ) \\
\hline$\overline{\text { BIT2 }}$ & Output of bit2 (digital, OV or $\left.V_{D D}\right)$ \\
\hline BIT3 & Output of bit3 (digital, OV or $V_{D D}$ ) \\
\hline VSS & GND (0V) \\
\hline$\overline{\mathrm{BIT} 4}$ & Output of bit4 (digital, OV or $V_{D D}$ ) \\
\hline BIT5 & Output of bit5 (digital, $0 \mathrm{~V}$ or $V_{D D}$ ) \\
\hline BIT6 & Output of bit6 (digital, OV or $V_{D D}$ ) \\
\hline BIT7 & Output of bit7 (digital, OV or $\left.V_{D D}\right)$ \\
\hline compare_select & Selects stage 1 or stage 2 for comparator output (0V or $\left.V_{D D}\right)$ \\
\hline VSS & GND (0V) \\
\hline Ibias & $\begin{array}{l}\text { Sets current bias for comparator (current between } 100 \mathrm{nA} \text { to } \\
1 \mu \mathrm{A} \text { ) }\end{array}$ \\
\hline Vref & Reference voltage for ADC (typically $V_{D D}$ ) \\
\hline Vtune & Adjusts offset voltage of comparator (typically 0V) \\
\hline analog_in & $\begin{array}{l}\text { Analog input into ADC (analog voltage between } 0 \text { and } \\
V_{R E F} \text { ) }\end{array}$ \\
\hline VDD_analog & $V_{D D}$ to supply analog components \\
\hline VSS & GND (0V) \\
\hline clk & clock for digital circuitry (sinusoid between 0 and $V_{D D}$ ) \\
\hline reset & resets digital logic, active on negative edge, active low. \\
\hline VDD & VDD to suppply digital components \\
\hline VDD_comparator_test & $V_{D D}$ for individual tested comparator \\
\hline Output_comparator_test & Output of individual tested comparator (digital, $0 \mathrm{~V}$ or $V_{D D}$ ) \\
\hline VDD_buffers & $V_{D D}$ to supply output buffers \\
\hline Input_comparator_test & $\begin{array}{l}\text { Input of individual tested comparator (analog, }-V_{D D} \text { to } \\
\left.V_{D D}\right)\end{array}$ \\
\hline
\end{tabular}




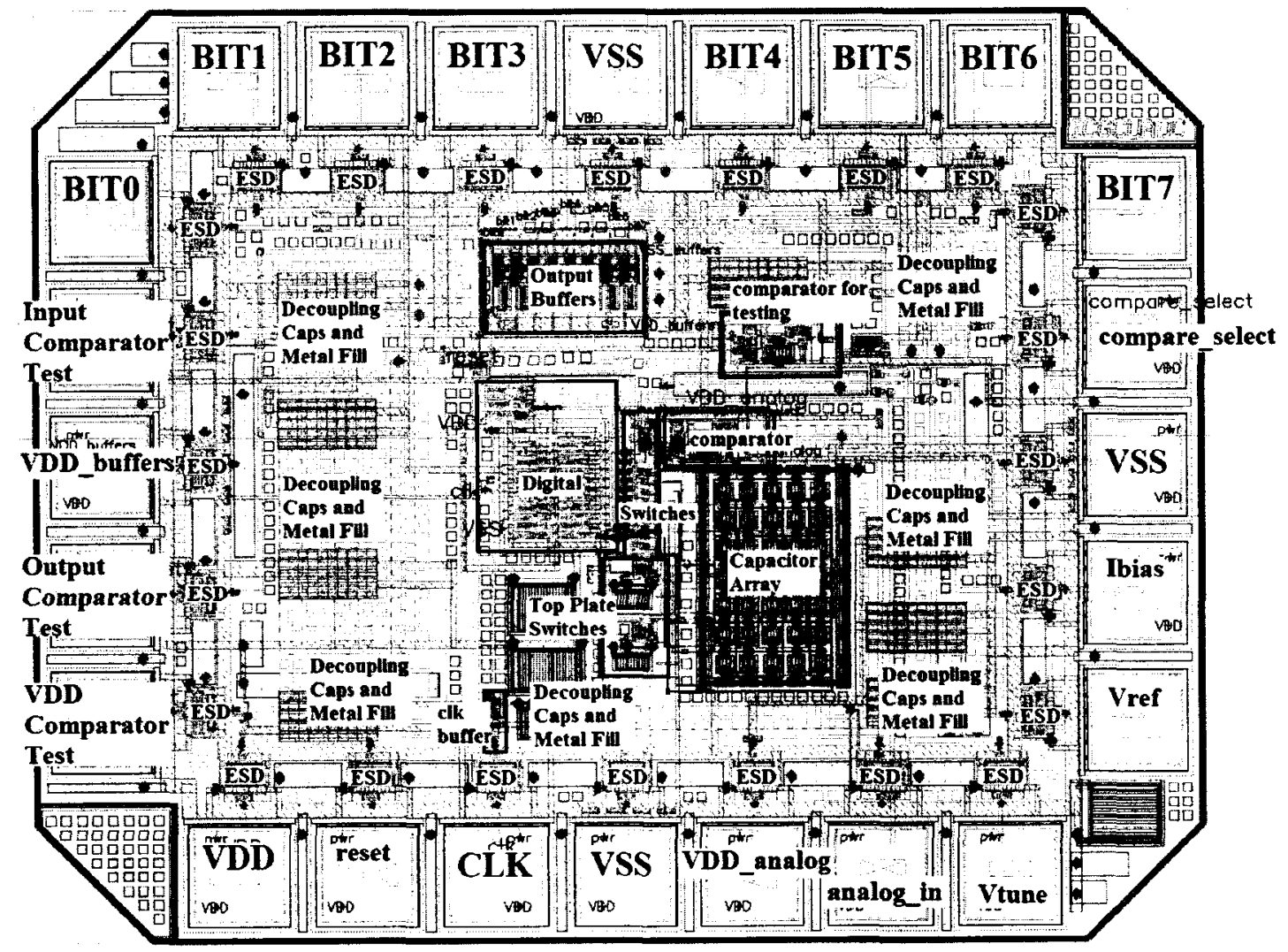

Figure 4.40: The final layout of ICGCUADC. Chip dimensions are $1.25 \mathrm{~mm}$ by $1.0 \mathrm{~mm}$, with an area of $1.25 \mathrm{~mm}^{2}$ and a core area of $0.125 \mathrm{~mm}^{2}$ 


\subsubsection{Chip 2: ICGCUKAH}

The second ADC chip, entitled ICGCUKAH, is $1.25 \mathrm{~mm}$ by $1.0 \mathrm{~mm}$ and its final layout is shown in Figure 4.41. It includes the ADC core of Figure 4.38, with additional bond pads, a separate comparator to be tested individually from the ADC, metal fill, clock buffers, ESD protection (resistors and diodes), decoupling capacitors and guard rings to isolate the analog components from the rest of the circuitry. All the pins of Figure 4.41 are described in detail in Table 4.7. It is important to note that the pad frames of ICGCUKAH and ICGCUADC were made as similar to each other as possible to simplify the PCB design and testing. 
Table 4.7: Summary of ICGCUKAH Pins.

\begin{tabular}{|c|c|}
\hline Name & Description \\
\hline BIT0 & Output of bit0 (digital, $0 \mathrm{~V}$ or $V_{D D}$ ) \\
\hline$\overline{\text { BIT1 }}$ & Output of bit1 (digital, $0 \mathrm{~V}$ or $V_{D D}$ ) \\
\hline$\overline{\text { BIT2 }}$ & Output of bit2 (digital, $0 \mathrm{~V}$ or $V_{D D}$ ) \\
\hline BIT3 & Output of bit3 (digital, $0 \mathrm{~V}$ or $V_{D D}$ ) \\
\hline VSS & GND (0V) \\
\hline BIT4 & Output of bit4 (digital, $0 \mathrm{~V}$ or $V_{D D}$ ) \\
\hline BIT5 & Output of bit5 (digital, $0 \mathrm{~V}$ or $V_{D D}$ ) \\
\hline BIT6 & Output of bit6 (digital, $0 \mathrm{~V}$ or $V_{D D}$ ) \\
\hline$\overline{\text { BIT7 }}$ & Output of bit7 (digital, $0 \mathrm{~V}$ or $V_{D D}$ ) \\
\hline compare_select & Selects stage 1 or stage 2 for comparator output ( $0 \mathrm{~V}$ or $\left.V_{D D}\right)$ \\
\hline Output_comparator_test & Output of individual tested comparator (digital, $0 \mathrm{~V}$ or $V_{D D}$ ) \\
\hline Ibias & $\begin{array}{l}\text { Sets current bias for comparator (current between } 100 \mathrm{nA} \text { to } \\
1 \mu \mathrm{A} \text { ) }\end{array}$ \\
\hline analog_GND & Reference analog ground for ADC $\left(V_{D D} / 2\right)$ \\
\hline Vtune & Adjusts offset voltage of comparator (typically 0V) \\
\hline analog_in & $\begin{array}{l}\text { Analog input into ADC (analog voltage between } 0 \text { and } \\
V_{R E F} \text { ) }\end{array}$ \\
\hline VDD_analog & $V_{D D}$ to supply analog components \\
\hline VSS & GND (0V) \\
\hline $\mathrm{clk}$ & clock for digital circuitry (sinusoid between 0 and $V_{D D}$ ) \\
\hline reset & resets digital logic, active on negative edge, active low. \\
\hline VDD & $V_{D D}$ to suppply digital components \\
\hline C1 & digital control 1 of tunable capacitor ( $0 \mathrm{~V}$ or $\left.V_{D D}\right)$ \\
\hline $\mathrm{C} 2$ & digital control 2 of tunable capacitor $\left(0 \mathrm{~V}\right.$ or $\left.V_{D D}\right)$ \\
\hline VDD_buffers & $V_{D D}$ to supply output buffers \\
\hline C3 & digital control 3 of tunable capacitor (0V or $\left.V_{D D}\right)$ \\
\hline
\end{tabular}




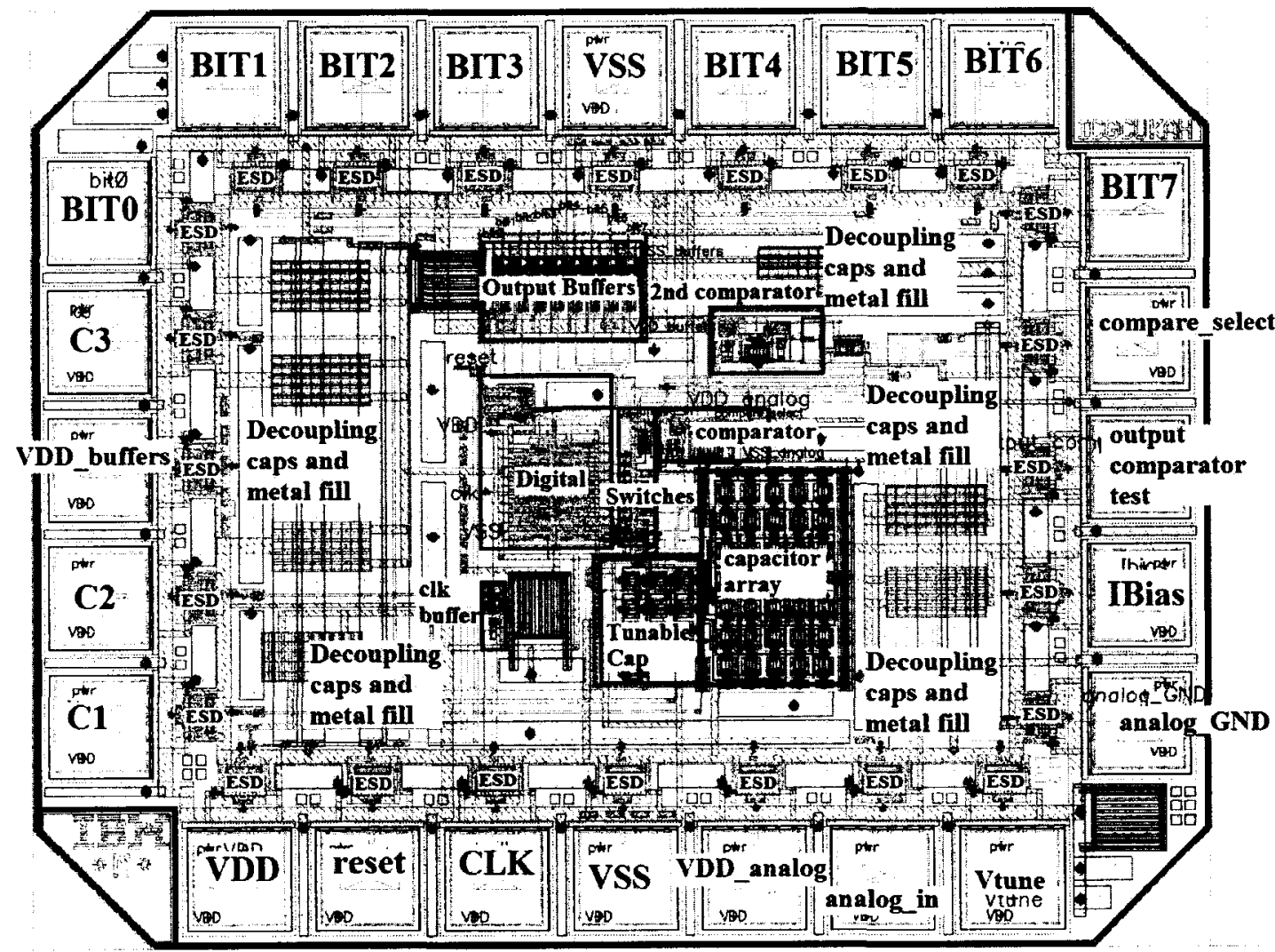

Figure 4.41: The final layout of ICGCUKAH. Chip dimensions are $1.25 \mathrm{~mm}$ by $1.0 \mathrm{~mm}$, with an area of $1.25 \mathrm{~mm}^{2}$ and a core area of $0.125 \mathrm{~mm}^{2}$. 


\section{Chapter 5}

\section{Simulations}

\subsection{Simulation Results of Analog to Digital Con- verter ICGCUADC}

Simulations for the first ADC ICGCUADC were all post-layout simulations with extracted * parasitic capacitors using Spectre as the simulator with full BSIM4 models. The final layout was simulated which includes ESD protection circuitry, bond pads and decoupling capacitors. Off-chip components such as $3 \mathrm{nH}$ bond wires and off-chip decoupling capacitors were included in the simulation.

\subsubsection{Linearity Results: INL and DNL}

A slow ramp input from 0 to $V_{R E F}$ was input into the $\mathrm{ADC}$, with a rise time of $60 \mathrm{~ms}$ sampled at $60 \mathrm{kS} / \mathrm{s}$, which allows for 3600 samples, or about 14 points per code. Figure 5.1 shows the digital output versus the analog input. All 256 codes are present so there are no missing codes for the ADC. Using the best-fit line for INL, the maximum INL is determined to be $0.26 \mathrm{LSB}$ as shown in Figure 5.2a.

The maximum DNL is determined to be 0.35LSB as shown in Figure 5.2b. A DNL of less than 1 LSB guarantees a monotonic transfer function, with no missing

${ }^{*}$ Cadence DIVA with the IBM $0.13 \mu \mathrm{m}$ kit 1.3.0.2. 


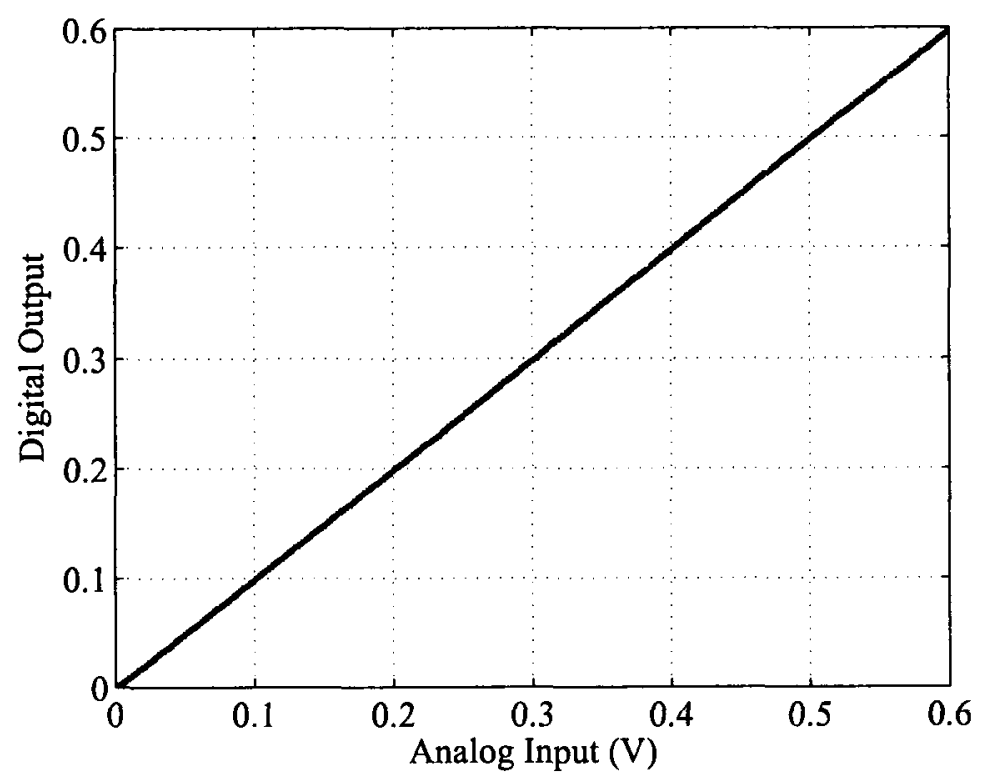

Figure 5.1: Digital output versus analog input for ICGCUADC at $60 \mathrm{kS} / \mathrm{s}$ with Vref $=0.6$ and $V_{D D}=0.6 \mathrm{~V}$.

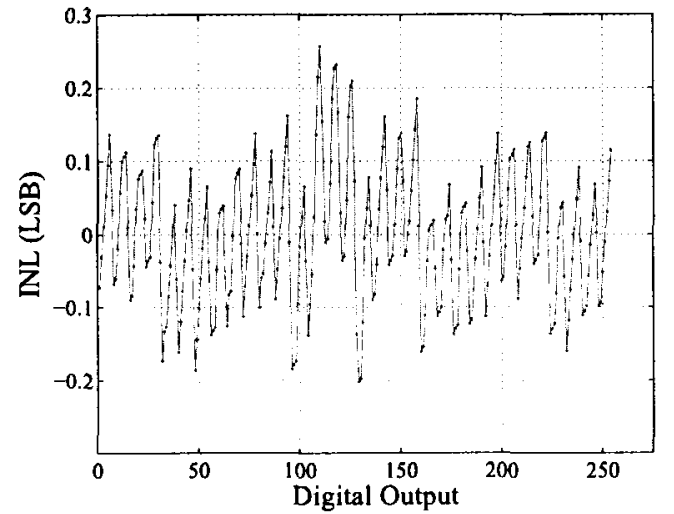

(a)

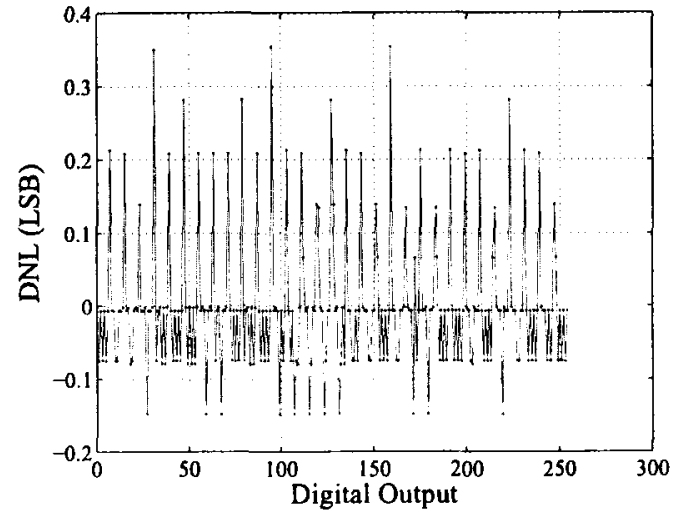

(b)

Figure 5.2: (a) INL plot for ICGCUADC with a sample rate of $60 \mathrm{kS} / \mathrm{s}$, Vref $=0.6$ and $V_{D D}=0.6 \mathrm{~V}$. (b) DNL plot for chip ICGCUADC with a sample rate of $60 \mathrm{kS} / \mathrm{s}$, Vref $=0.6$ and $V_{D D}=0.6 \mathrm{~V}$. 
codes [34]. Variations in capacitor mismatch generally degrade INL and DNL for the measured ADC, however, through the use of the common centroid layout; unit capacitors; and a dummy capacitor ring, the capacitors should be matched closely.

\subsubsection{Dynamic Results: SFDR, SINAD and ENOB}

To simulate the dynamic performance of the ADC a sinusoidal signal with an amplitude from 0 to $V_{R E F}$ was input into the ADC. For different simulations, different frequencies were input from about $0.001 \times$ sample rate to about $0.3 \times$ sample rate. The digital bits that were output from the ADC were then input into an ideal Analog Hardware Description Language (AHDL) DAC converter, which converts the bits back to an analog voltage. The DFT was taken on the new analog output, and specifications such as the Spurious Free Dynamic Range (SFDR), Signal-to-Noise and Distortion Ratio (SINAD) and the effective number of bits (ENOB) were extracted from the DFT. The SINAD as described in [35] is the ratio in $\mathrm{dB}$ between the signal power and the noise power including harmonics below the Nyquist frequency except the DC component. The SFDR as described in [35] is the ratio in $\mathrm{dB}$ between the signal power and the next highest harmonic below the Nyquist frequency excluding the DC component.

A plot of SINAD and SFDR vs. input frequency is shown in Figure 5.3a for the case when the supply voltage is $0.4 \mathrm{~V}$, Figure $5.3 \mathrm{~b}$ for the case when the supply voltage is $0.6 \mathrm{~V}$ and Figure $5.3 \mathrm{c}$ when the supply is $0.8 \mathrm{~V}$. An ideal 8 -bit ADC would have a SINAD of $49.92 \mathrm{~dB}$ from $\mathrm{DC}$ to the Nyquist frequency. At low frequencies (10-100 times below the sampling rate of the ADC), the ADC achieves close to the 
ideal SINAD for the 3 supply voltage cases. In Figure 5.4 the SINAD is shown for all three supply voltage cases, when the input frequency is normalized by dividing it by the Nyquist frequency. When a higher $V_{D D}$ is used a higher linearity is achieved for the analog switches during the sampling and holding phase of the ADC. As the ADC is operating almost entirely in the sub-threshold regime $\left(V_{D D}=0.4 \mathrm{~V}\right)$, the switches introduce more distortion and lower the bandwidth of the ADC. Also, with a lower supply voltage there is more delay in the digital control signals which can cause significant time between the sampling clock to when the input signal is held for conversion. This can add noise at higher input frequencies where the input changes more drastically during this delay. For a lower supply voltage and reference voltage the LSB decreases as well, which increases the accuracy requirements of the comparator and capacitors, introducing more harmonic distortion and lowering the SINAD of the ADC.

The ENOB as described in [36] is a measure of how close the ADC performs against an ideal $\mathrm{ADC}$, and is given by the following equation,

$$
\mathrm{ENOB}=\frac{\operatorname{SINAD} d B-1.76 d B}{6.02}
$$

A plot of ENOB versus input frequency is shown in Figure 5.5a for the case when the supply voltage is $0.4 \mathrm{~V}$, Figure $5.5 \mathrm{~b}$ for the case when the supply voltage is $0.6 \mathrm{~V}$, and Figure $5.5 \mathrm{c}$ when the supply is $0.8 \mathrm{~V}$. All three cases have an ENOB of 8-bits at low frequencies and taper off as the input frequency increases, similar to the SINAD.

A Monte Carlo simulation with process and mismatch variation was performed for the case when the input frequency is $5 \mathrm{kHz}$, sampled at $60 \mathrm{kS} / \mathrm{s}$ while using a 


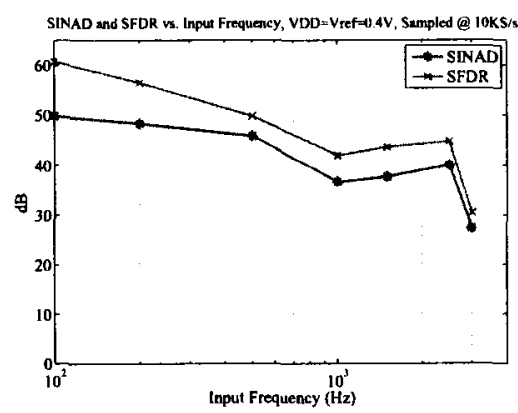

(a)

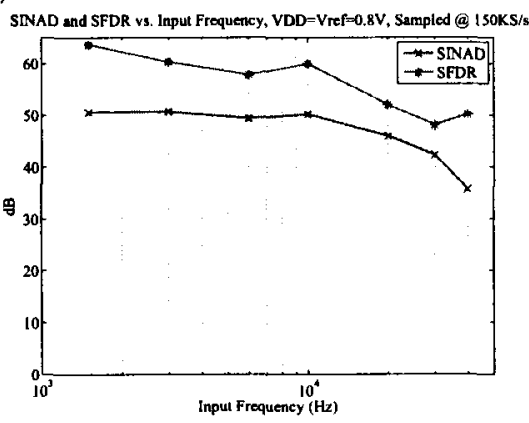

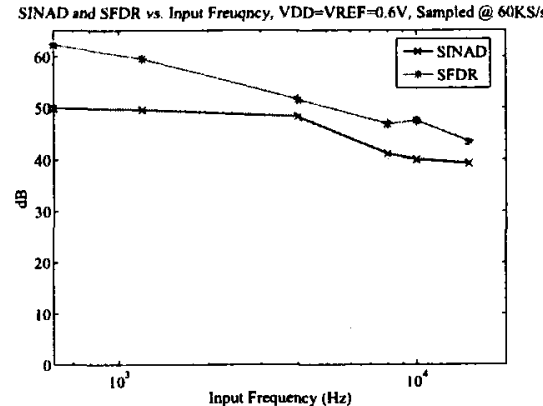

(b)

(c)

Figure 5.3: (a) Signal-to-Noise and Distortion (SINAD) and Spurious Free Dynamic Range (SFDR) vs. input frequency when $V_{D D}=V_{R E F}=0.4 \mathrm{~V}$, and a sample rate of 10kS/s. (b) Signal-to-Noise and Distortion (SINAD) and Spurious Free Dynamic Range (SFDR) vs. input frequency when $V_{D D}=V_{R E F}=0.6 \mathrm{~V}$, and a sample rate of $60 \mathrm{kS} / \mathrm{s}$. (c) Signal-to-Noise and Distortion (SINAD) and Spurious Free Dynamic Range (SFDR) vs. input frequency when $V_{D D}=V_{R E F}=0.8 \mathrm{~V}$, and a sample rate of $150 \mathrm{KkS} / \mathrm{s}$. 


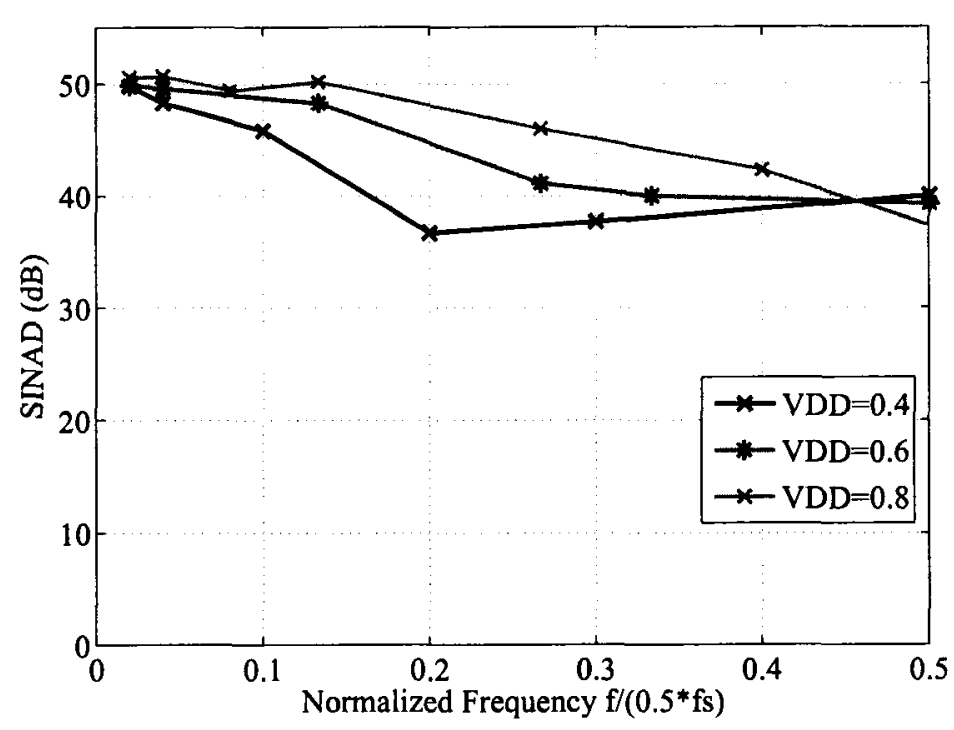

Figure 5.4: Signal-to-Noise and Distortion (SINAD) vs. normalized frequency when $V_{D D}=V_{R E F}=0.8 \mathrm{~V}, 0.6 \mathrm{~V}, 0.4 \mathrm{~V}$

supply voltage of $0.6 \mathrm{~V}$. Only 10 iterations* were performed as it was a demanding simulation in terms of computer simulation time. The 10 iterations did not take into account any of the common centroid matching which was used for the capacitors and the comparator which would give pessimistic mismatch for these components. The result is shown in Figure 5.6, where the nominal simulation (no process and mismatch variation) obtained 7 bits of accuracy. The average ENOB for the 10 iterations was 6.79 bits with the maximum being 7.65 bits and the minimum being 5.88 bits. The improved ENOB after process and mismatch variation is likely due to the switches obtaining faster switching transistors which would increase the bandwidth of the ADC.

*Not enough for statistical significance but enough to see the effect of process and mismatch variation on the $\mathrm{ADC}$. 


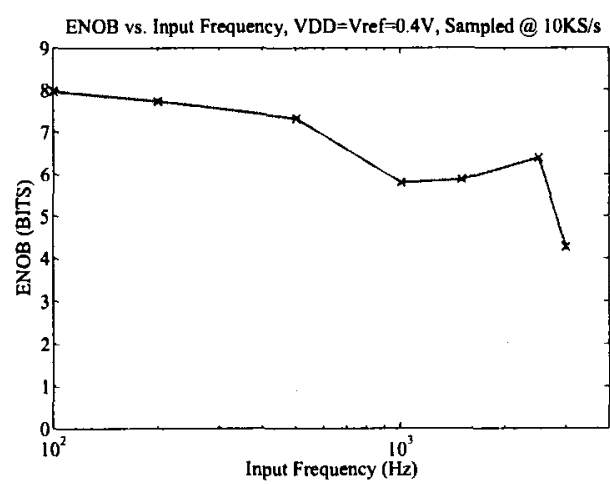

(a)

ENOB vs. Input Frequnecy, VDD=Vref=0.8V, Sampled @ 150KS/s

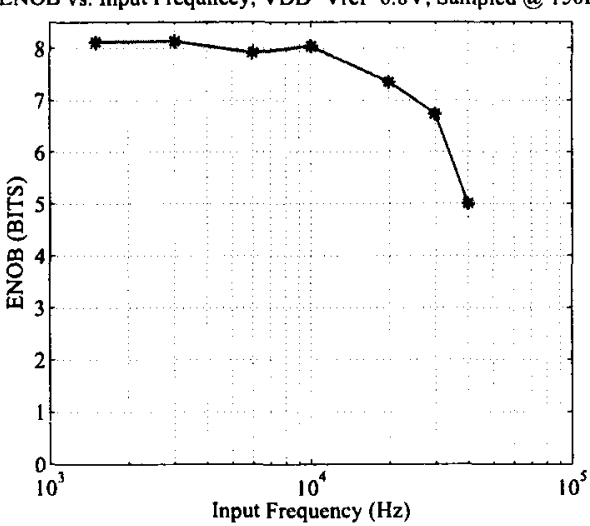

(c)

Figure 5.5: (a) Effective Number of Bits (ENOB) vs. input frequency when $V_{D D}=V_{R E F}=0.4 \mathrm{~V}$, and a sample rate of $10 \mathrm{kS} / \mathrm{s}$. (b) Effective Number of Bits (ENOB) vs. input frequency when $V_{D D}=V_{R E F}=0.6 \mathrm{~V}$, and a sample rate of $60 \mathrm{kS} / \mathrm{s}$. (c) Effective Number of Bits (ENOB) vs. input frequency when $V_{D D}=V_{R E F}=0.8 \mathrm{~V}$, and a sample rate of $150 \mathrm{kS} / \mathrm{s}$.

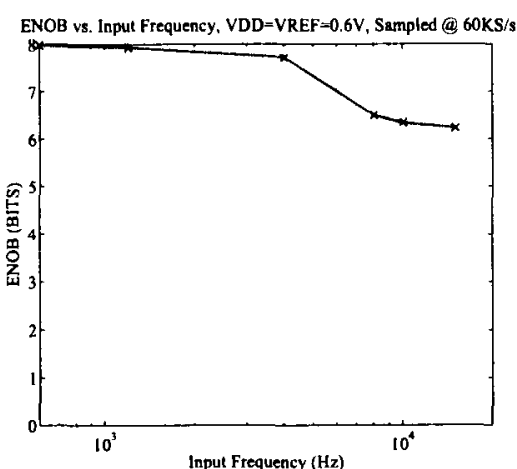

(b) 


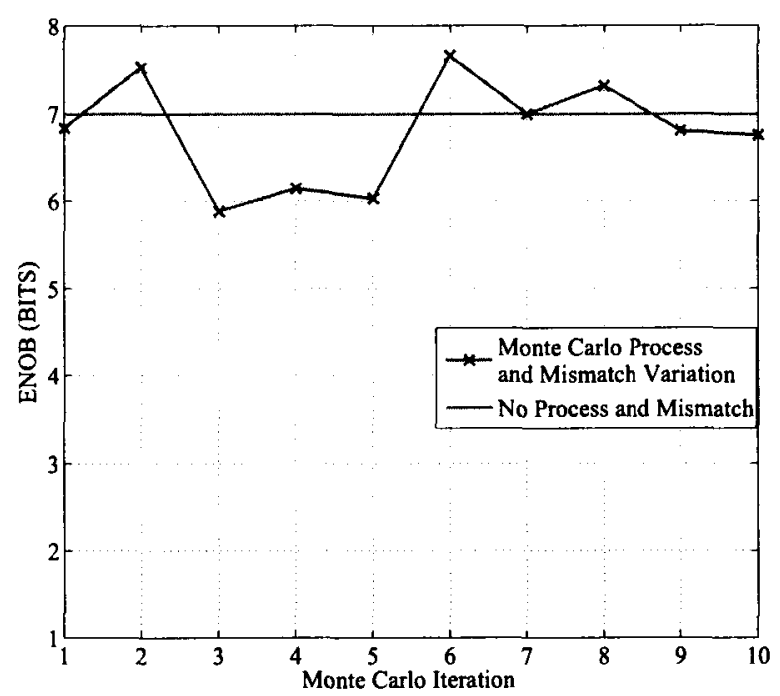

Figure 5.6: Effective Number of Bits (ENOB) vs. Monte Carlo Iteration when $V_{D D}=V_{R E F}=0.6 \mathrm{~V}$, input frequency of $5 \mathrm{KHz}$ and a sample rate of $60.3 \mathrm{kS} / \mathrm{s}$.

\subsubsection{Power Dissipation}

In Figure 5.7a the power dissipation of the full ADC core (excluding output buffers) is shown. The power increases exponentially with an increasing $V_{D D}$ up to the maximum for this process of $1.2 \mathrm{~V}$. As $V_{D D}$ increases generally higher sample rates can be achieved, as the comparator can achieve a higher bandwidth and the analog switches have a lower ON resistance. Taking into account the sample rate of the ADC a plot of energy per cycle is shown in Figure 5.7b. Operating with a lower $V_{D D}$ and in the sub-threshold region of operation the highest energy efficiency per conversion cycle is achieved. As we increase $V_{D D}$ the digital logic, capacitor array, and the capacitors for the comparator and the top switch has its power dissipation increase proportional to $V_{D D}^{2}$. For these components, the power dissipation also increases linearly as the sample rate is increased. Thus, there is a quadratic power dissipation increase with 


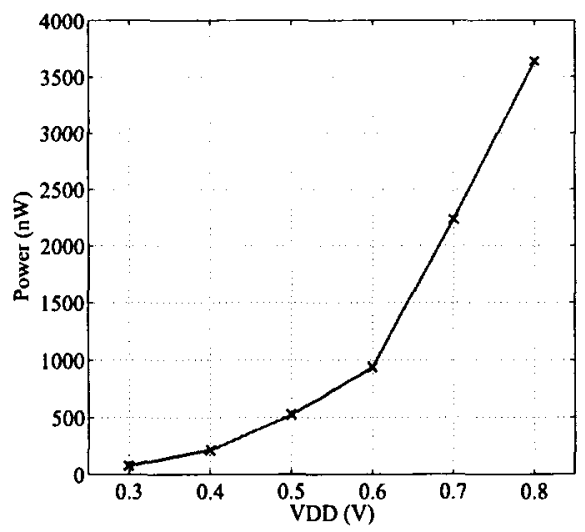

(a)

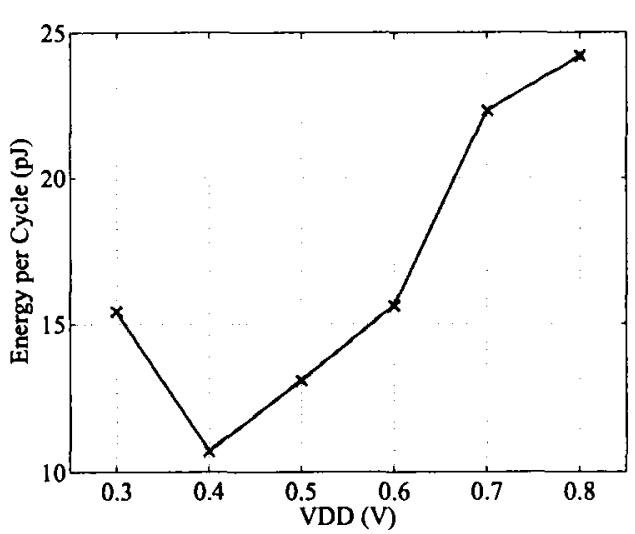

(b)

Figure 5.7: (a) Power dissipation of full ADC ICGCUADC vs. $V_{D D}$. Sample rate $=5 \mathrm{kS} / \mathrm{s}, 20 \mathrm{kS} / \mathrm{s}, 40 \mathrm{kS} / \mathrm{s}, 60 \mathrm{kS} / \mathrm{s}, 100 \mathrm{kS} / \mathrm{s}$ and $150 \mathrm{kS} / \mathrm{s}$ for $V_{D D}$ of $0.3,0.4,0.5,0.6,0.7$ and $0.8 \mathrm{~V}$ respectively. (b) Energy per cycle of full ADC ICGCUADC vs. $V_{D D}$. Sample rate $=5 \mathrm{kS} / \mathrm{s}, 20 \mathrm{kS} / \mathrm{s}, 40 \mathrm{kS} / \mathrm{s}, 60 \mathrm{kS} / \mathrm{s}, 100 \mathrm{kS} / \mathrm{s}$ and $150 \mathrm{kS} / \mathrm{s}$ for $V_{D D}$ of $0.3,0.4,0.5,0.6,0.7$ and $0.8 \mathrm{~V}$ respectively.

an increase in supply voltage and a linear power dissipation increase as the sample rate is increased.

The lowest simulated energy per conversion cycle was determined to be $11 \mathrm{pJ} / \mathrm{cycle}$ when $V_{D D}$ was $0.4 \mathrm{~V}$. Further decreasing of $V_{D D}$ significantly decreases the bandwidth as the switches have a very high ON resistance and the energy efficiency is degraded. A reasonable $V_{D D}$ for this ADC in terms of energy efficiency should be between 0.3 and $0.6 \mathrm{~V}$. Further increasing of $V_{D D}$ results in many of the transistors operating in the saturation region and more energy is required per conversion cycle. It should be noted from the previous section that there is a power versus bandwidth trade-off. Dynamic performance is improved when operating with a higher $V_{D D}$, but the ADC is less energy efficient. Thus, the nominal $V_{D D}$ selected for the ADC was $0.6 \mathrm{~V}$ which provides the best balance between energy efficiency and dynamic performance. 
In Figure $5.8 \mathrm{a}$ the $V_{D D}$ is kept at $0.6 \mathrm{~V}$ and the sample rate is increased between $10 \mathrm{kS} / \mathrm{S}$ and $100 \mathrm{kS} / \mathrm{s}$. The majority of power is used by the analog components. Without offset correction circuitry and a full range switch to reset the top of the capacitor array the analog power would be approximately constant with sample rate. However, the offset correction circuitry has a capacitor to store the offset voltage and the two switches to reset the top plate of the capacitor array have two capacitors. These three capacitors are charged and discharged each conversion cycle which result in a linear increase in power dissipation with an increasing sample rate. At low sample rates $(10 \mathrm{kS} / \mathrm{s})$ the analog circuitry contributes $93 \%$ of the total power dissipation. At higher sample rates $(100 \mathrm{kS} / \mathrm{s})$ the analog circuitry contributes $74 \%$ of the total power dissipation. The power dissipation of the digital logic and capacitor array is increasing higher than the analog components with an increasing sample rate. An optimum simulated sample rate can also be found by plotting energy per conversion versus sample rate as shown in Figure 5.8b. The optimum sample rate was found to be approximately $90 \mathrm{kS} / \mathrm{s}$ where the energy per conversion cycle is $12.7 \mathrm{pJ} /$ cycle. At lower sample rates the comparator is operating at a bandwidth higher than necessary for this sample rate (constant $I_{\text {bias }}$ ) and at higher sample rates, the dynamic digital and $V_{R E F}$ power become more significant. For such low sample rates, a lower $V_{D D}$ should be used.

\subsubsection{Temperature}

Temperature was swept from -30 to 150 degrees Celsius and the full ADC was simulated. The input was set to $V_{D D} / 2$ where the output should be 01111111 . The output 


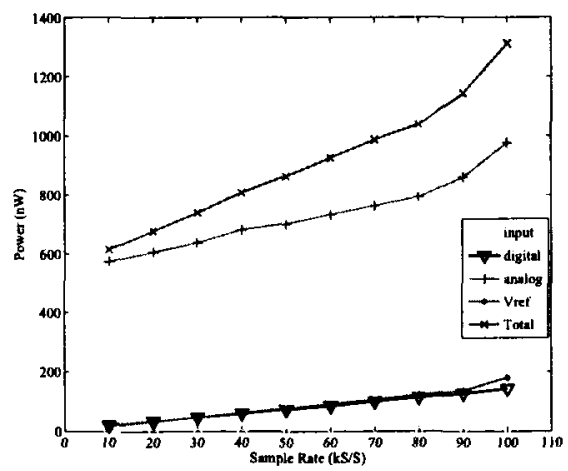

(a)

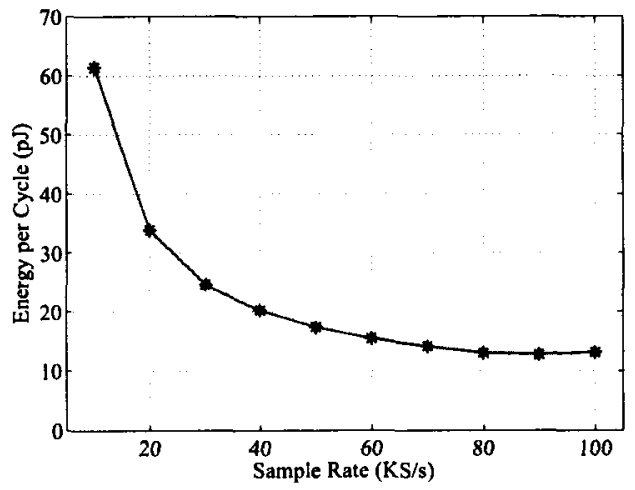

(b)

Figure 5.8: (a) Power dissipation of full ADC ICGCUADC vs. sample rate for $V_{D D}=0.6 \mathrm{~V}$. (b) Energy per cycle of full ADC ICGCUADC vs. sample rate for $V_{D D}=0.6 \mathrm{~V}$.

was correct for between -10 to 100 degrees Celsius. Outside this temperature range the comparator fails to function properly as its offset voltage becomes much more significant, leading to errors for the ADC output. The result is shown in Figure 5.9a. Looking at the power dissipation with respect to temperature, there is an increasing quadratic power relationship as shown in Figure $5.9 \mathrm{~b}$. The comparator being biased in sub-threshold increases in current as temperature is increased. When operating with a lower $V_{D D}$ the $\mathrm{ADC}$ is more sensitive to temperature variation and operating with a higher $V_{D D}$ it is less sensitive to temperature.

\subsubsection{Monte Carlo Results}

Monte Carlo statistical analysis was performed for the entire ADC, with an input of $V_{D D} / 2$, and the digital output and power dissipation were examined. Process and mismatch variation were enabled for the models with no matching for the transistors leading to a pessimistic analysis. The first case was when the ADC had a supply 


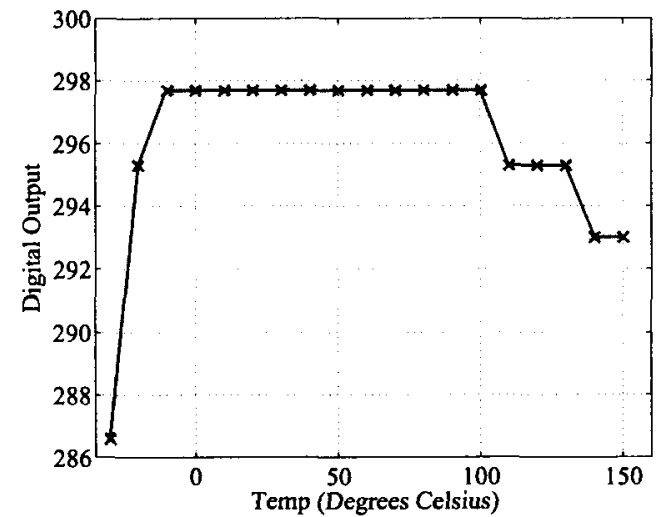

(a)

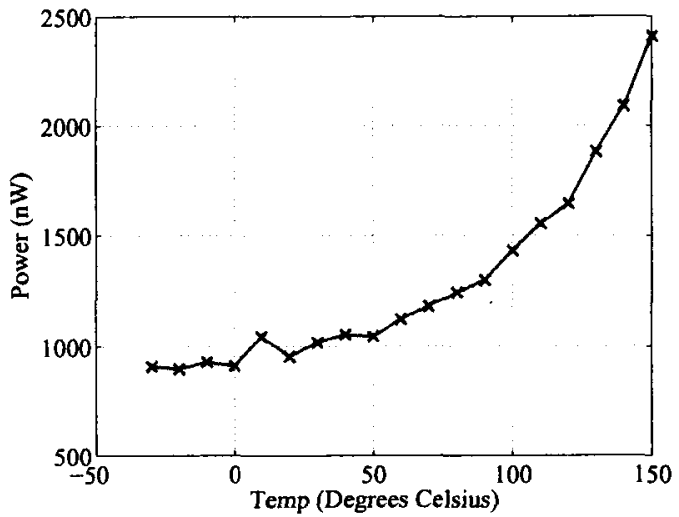

(b)

Figure 5.9: (a) Digital output of full ADC ICGCUADC vs. temperature when analog input is set to $300 \mathrm{mV}, V_{D D}=0.6 \mathrm{~V}$, sample rate $=60 \mathrm{kS} / \mathrm{s}$. (b) Power dissipation of full ADC ICGCUADC vs. temperature for $V_{D D}=0.6 \mathrm{~V}$, sample rate $=60 \mathrm{kS} / \mathrm{s}$.

voltage of $0.4 \mathrm{~V}$ (sample rate of $5 \mathrm{kS} / \mathrm{s}$ ) with the results shown in Figure 5.10a. For the 50 iterations the output ranged from $193.8 \mathrm{mV}$ to $201.6 \mathrm{mV}$ with an average output of $198.1 \mathrm{mV}$ and a standard deviation of $1.3 \mathrm{mV}$ or 0.832 LSBs. The output was correct 37 times out of the 50 runs. For power dissipation, it ranged from $133.8 \mathrm{nW}$ to $184.1 \mathrm{nW}$ with an average of $161.1 \mathrm{nW}$ and a standard deviation of $11.2 \mathrm{nW}$. At a low supply voltage the ADC becomes more sensitive to comparator offset and capacitor mismatch as a result of mismatch from the comparator and capacitors respectively.

The second case was when the ADC had a supply voltage of $0.6 \mathrm{~V}$ (sample rate of $60 \mathrm{kS} / \mathrm{s}$ ) with the results shown in Figure $5.10 \mathrm{~b}$. For the 50 iterations the output ranged from $295.3 \mathrm{mV}$ to $300 \mathrm{mV}$ with an average output of $297.9 \mathrm{mV}$ and a standard deviation of $1.1 \mathrm{mV}$ or 0.469 LSBs. The output was correct 39 times out of the 50 runs. For power dissipation, it ranged from $925.2 \mathrm{nW}$ to $1131 \mathrm{nW}$ with an average of $1021 \mathrm{nW}$ and a standard deviation of $47.97 \mathrm{nW}$. The energy per conversion cycle is 

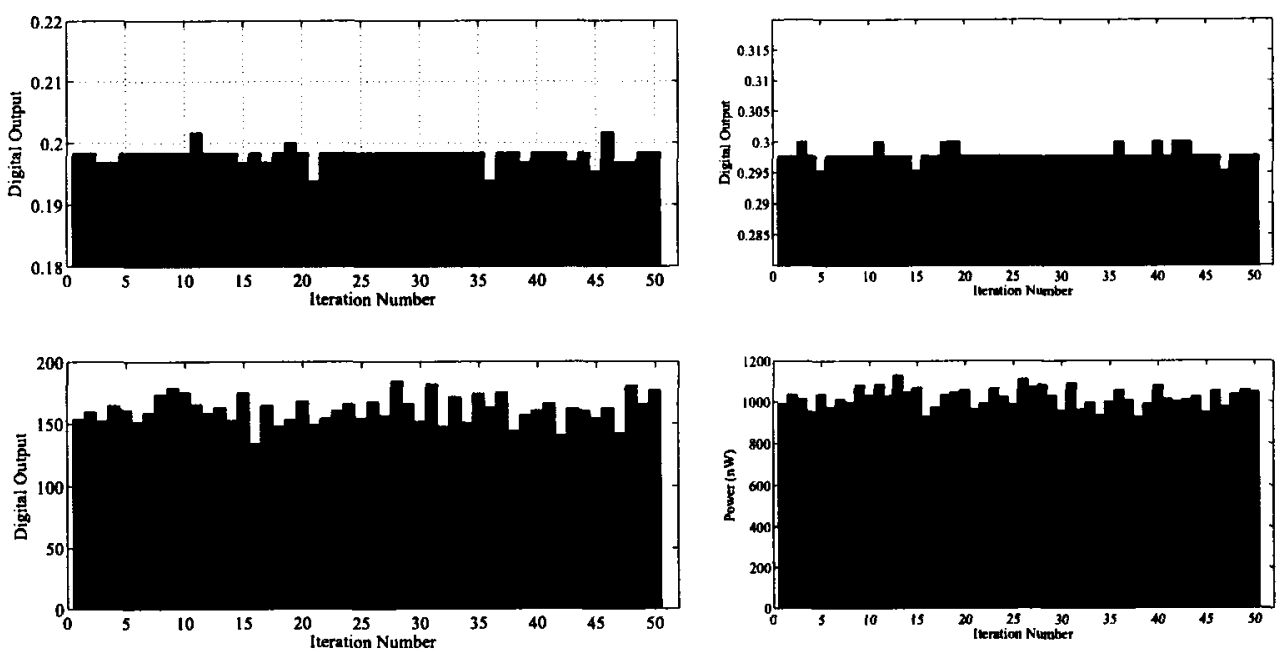

(a)

(b)
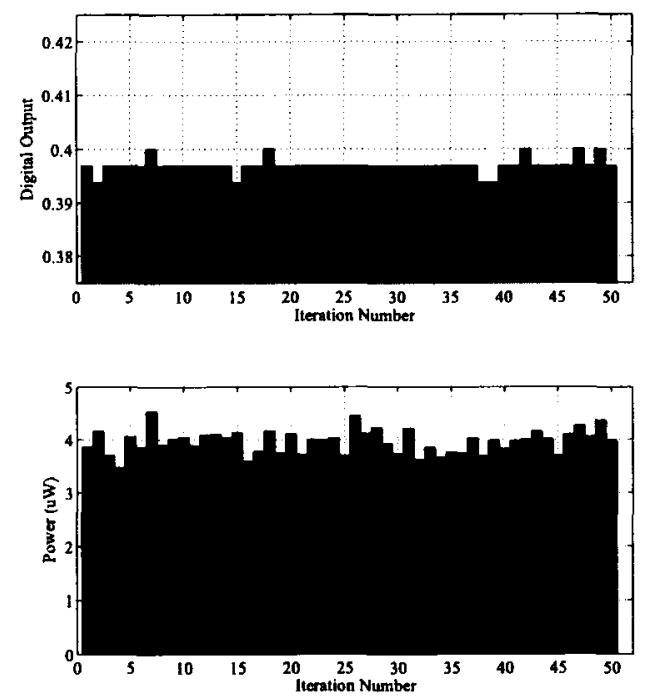

(c)

Figure 5.10: (a) Output and Power dissipation of full ADC ICGCUADC for 50 Monte Carlo iterations with process and mismatch for $V_{D D}=0.4 \mathrm{~V}, V_{I N}=0.2 \mathrm{~V}$, sample rate $=5 \mathrm{kS} / \mathrm{s}$.(b) Output and Power dissipation of full ADC ICGCUADC for 50 Monte Carlo iterations with process and mismatch for $V_{D D}=0.6 \mathrm{~V}, V_{I N}=0.3 \mathrm{~V}$, sample rate $=60 \mathrm{kS} / \mathrm{s}$. (c) Output and Power dissipation of full ADC ICGCUADC for 50 Monte Carlo iterations with process and mismatch for $V_{D D}=0.8 \mathrm{~V}, V_{I N}=0.4 \mathrm{~V}$, sample rate $=150 \mathrm{kS} / \mathrm{s}$. 
between $15.42 \mathrm{pJ} /$ sample and $18.85 \mathrm{pJ} /$ sample.

The last case was when the ADC had a supply voltage of $0.8 \mathrm{~V}$ (sample rate of $150 \mathrm{kS} / \mathrm{s}$ ) with the results shown in Figure 5.10c. For the 50 iterations the output ranged from $393.8 \mathrm{mV}$ to $400 \mathrm{mV}$ with an average output of $397 \mathrm{mV}$ and a standard deviation of $1.3 \mathrm{mV}$ or 416 LSBs. The output was correct 41 times out of the 50 runs. For power dissipation, it ranged from $3.48 \mu \mathrm{W}$ to $4.53 \mu \mathrm{W}$ with an average of $3.964 \mu \mathrm{W}$ and a standard deviation of $221 \mathrm{nW}$. The energy per conversion cycle is between $23.2 \mathrm{pJ} /$ sample and $30.2 \mathrm{pJ} /$ sample. The higher supply voltage allowed for a more robust response to process and mismatch variation as the LSB voltage is higher. This lowered the accuracy requirements of the comparator as a result of comparator offset, but capacitor mismatch still degraded ADC performance.

\subsection{Simulation Results of Analog to Digital Con- verter ICGCUKAH}

Simulations for the first ADC entitled, ICGCUKAH, were all post-layout simulations with parasitic capacitors using Spectre as the simulator with full BSIM4 models. The final layout was simulated which includes ESD protection circuitry, bond pads and decoupling capacitors. Off-chip components such as $3 \mathrm{nH}$ bond wires and off-chip decoupling capacitors were included in the simulation.

\subsubsection{Linearity Results: INL and DNL}

A slow ramp input from analog GND to $V_{D D}$ was input into the $\mathrm{ADC}$, with a rise time of $60 \mathrm{~ms}$ sampled at $60 \mathrm{kS} / \mathrm{s}$, which allows for 3600 samples, or about 14 points per code. Figure 5.11 shows the digital output versus the analog input. All 256 


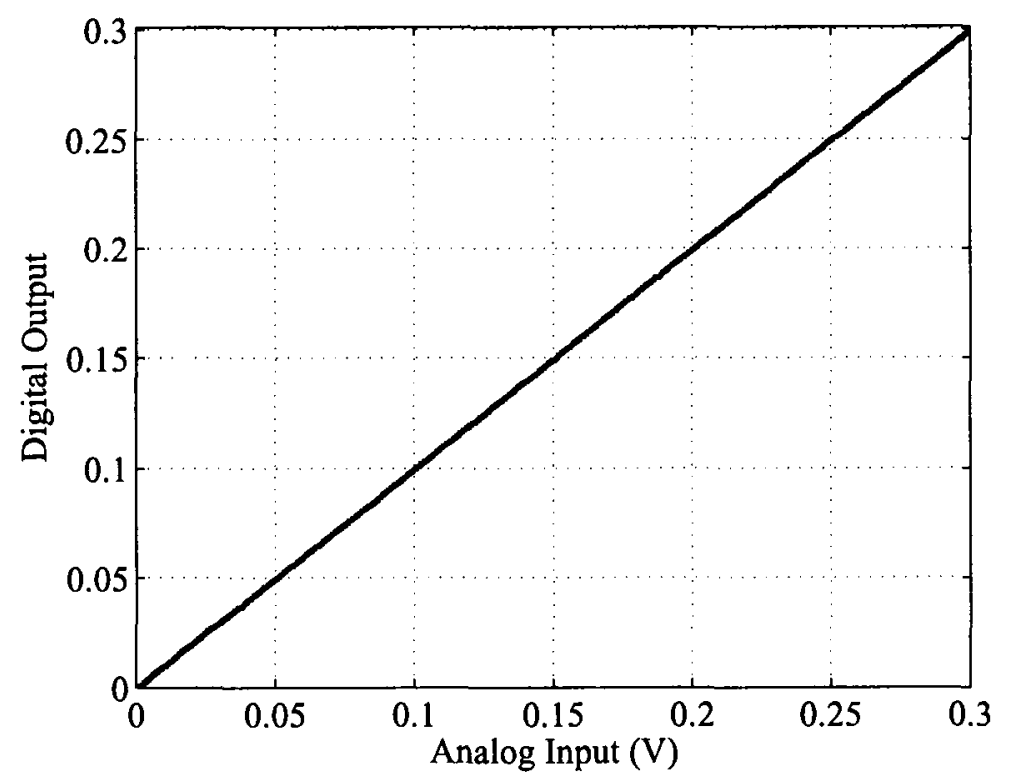

Figure 5.11: Digital output versus analog input for ICGCUKAH at $60 \mathrm{kS} / \mathrm{s}$ with Vref $=0.3$ and $V_{D D}=0.6 \mathrm{~V}$.

codes are present, allowing for no missing codes for the ADC. This case used the optimum capacitor value when $C_{1} C_{2} C_{3}$ is set to '100'. Using the best-fit line for INL, the maximum INL is determined to be $0.382 \mathrm{LSB}$ as shown in Figure 5.12a. The maximum DNL is determined to be $0.833 \mathrm{LSB}$ as shown in Figure 5.12b. A DNL of less than 1 LSB guarantees a monotonic transfer function, with no missing codes [34]. The DNL is approximately double the case of the first ADC as the input range is half.

\subsubsection{Effect of Tuning on Linearity}

A post layout simulation * was performed for the 32 codes centered at the switching point between 01111111 to 10000000 (Vref/2) and the sum of DNLs were found for

${ }^{*}$ Cadence DIVA with the IBM $0.13 \mu \mathrm{m}$ kit 1.3.0.2. 


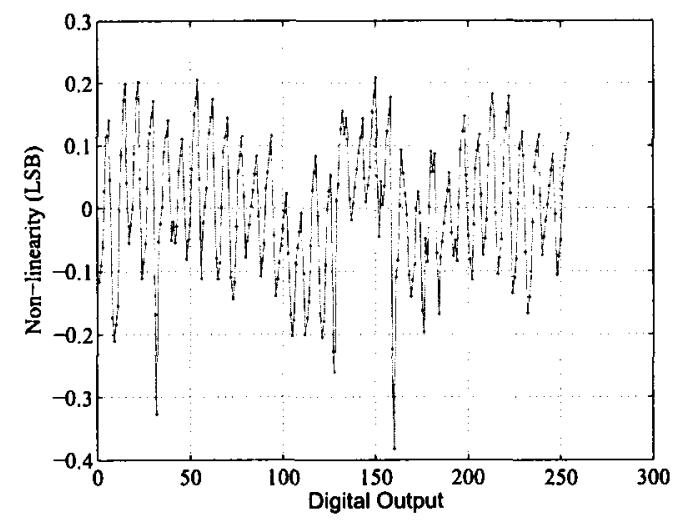

(a)

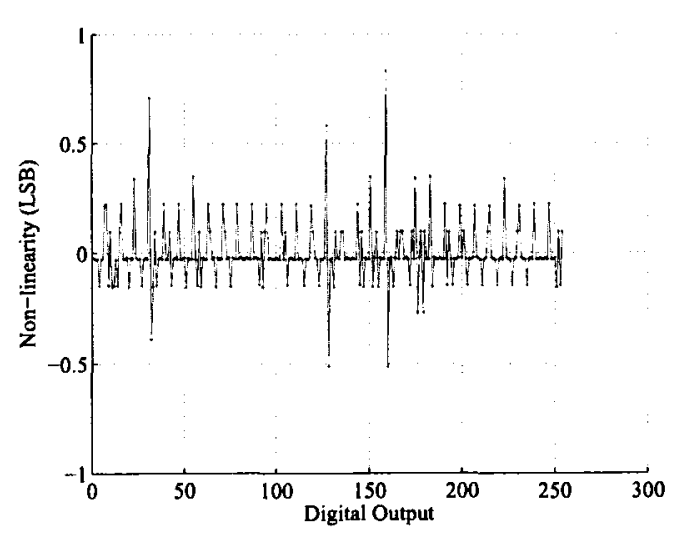

(b)

Figure 5.12: (a) INL plot for ICGCUKAH with a sample rate of $60 \mathrm{kS} / \mathrm{s}, \mathrm{Vref}=0.3$ and $V_{D D}=0.6 \mathrm{~V}$. (b) DNL plot for ICGCUKAH with a sample rate of $60 \mathrm{kS} / \mathrm{s}, \mathrm{Vref}=0.3$ and $V_{D D}=0.6 \mathrm{~V}$.

each value of the tunable capacitor. The result is shown in Figure 5.13 and shows the optimum value of capacitance for obtaining minimum DNL. This value was determined to be $C_{1} C_{2} C_{3}={ }^{\prime} 100$ '. This will achieve the most linear ADC response which is approximately at the center of the tuning range for the tunable capacitor.

\subsubsection{Effect of Tuning on Dynamic Performance}

A sine wave at different frequencies between $1 \mathrm{kHz}$ and $15 \mathrm{kHz}$ was input into the ADC sampling at $60 \mathrm{kS} / \mathrm{s}$ with a supply voltage of $0.6 \mathrm{~V}$. A plot of the effective number of bits (ENOB) for the 8 capacitor values at different input frequencies is shown in Figure 5.14. At low frequencies when the capacitor code is 100 as shown in Figure 5.14 one can achieve the maximum 8-bit accuracy. For the two extreme cases, when the capacitor code is 000 and 111 , leads to the least accurate ADC.

In Figure 5.15 the average ENOB is taken over all frequencies from $1 \mathrm{kHz}$ to $15 \mathrm{kHz}$ and plotted for each capacitor value. Similar to the case for linearity, setting 


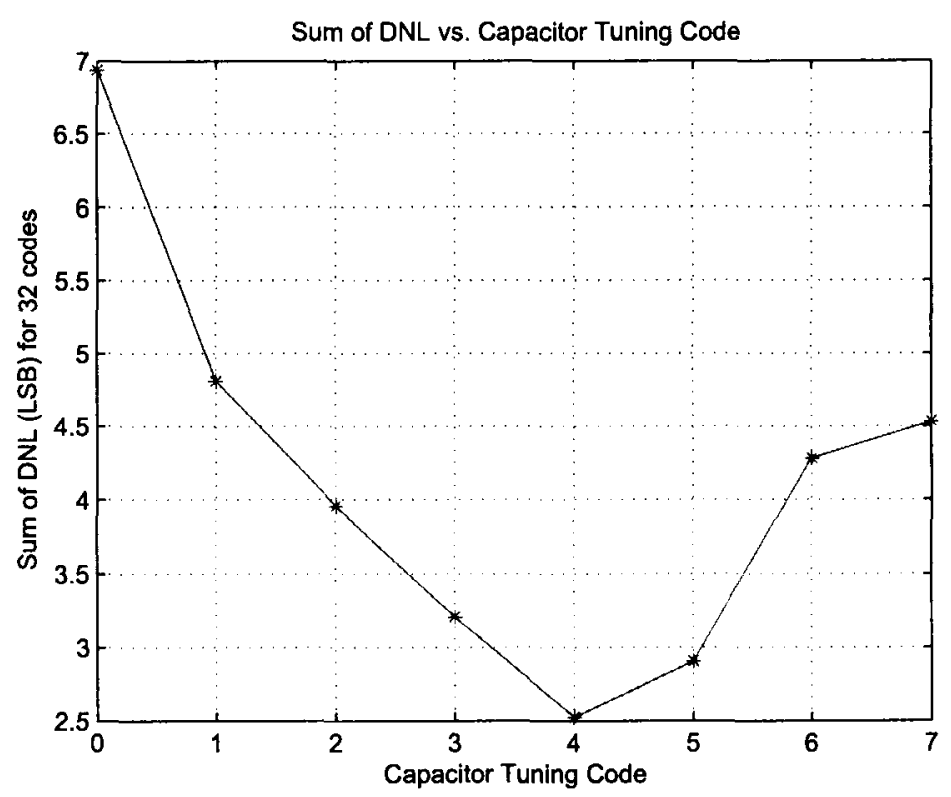

Figure 5.13: Sum of DNL for 32 codes for each capacitor value with a Vref $=0.3, V_{D D}=0.6$, sample rate $=50 \mathrm{kS} / \mathrm{s}$.

the capacitor to $C_{1} C_{2} C_{3}=$ ''100' will enable the most accurate ADC. The two extreme cases $C_{1} C_{2} C_{3}=' 000$ ' and $C_{1} C_{2} C_{3}=$ ''111' enables the least accurate ADC for all tunable values of the ADC. The optimum capacitor case offers better accuracy than the other seven capacitor cases both for linearity and dynamic performance for the ADC. The improvement could be more significant if the ADC had more bits (nine or more) as the LSB is smaller making the ADC more sensitive to the value of the attenuation capacitor or there was more capacitor values within the tuning range.

\subsubsection{Power Dissipation}

In Figure 5.16a and in Figure 5.16b the power dissipation of the full ADC core for ICGCUKAH (excluding output buffers) is shown for different supply voltages. The 


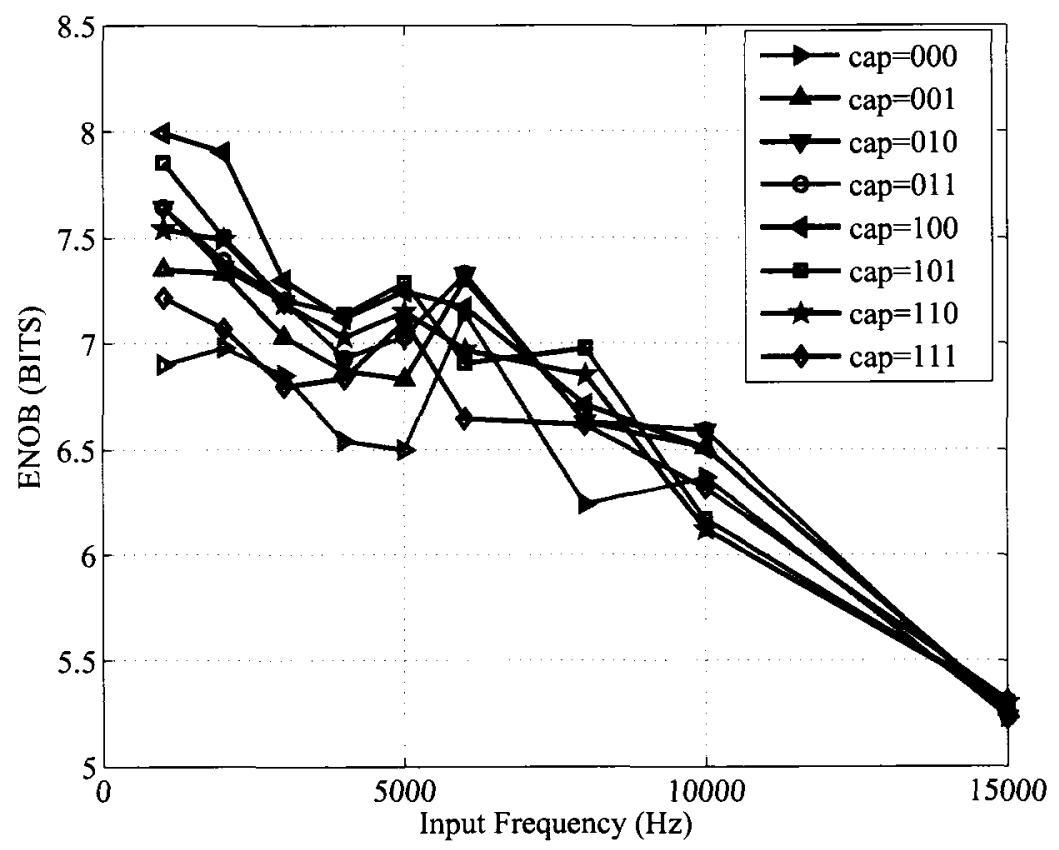

Figure 5.14: ENOB vs. Input frequency, for each capacitor value for $V_{D D}=0.6 \mathrm{~V}$, $V_{R E F}=0.3 \mathrm{~V}$, sample rate $=60 \mathrm{kS} / \mathrm{s}$.

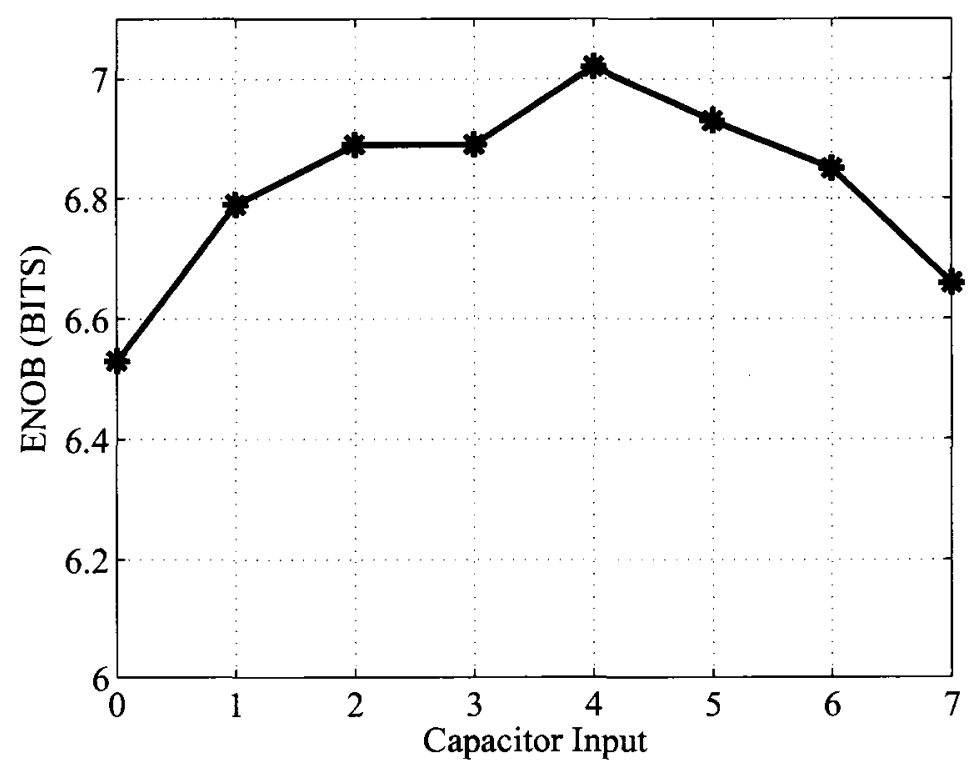

Figure 5.15: Average ENOB $(600 \mathrm{~Hz}$ to $15 \mathrm{KHz})$ from Figure 5.14 for each capacitor value for $V_{D D}=0.6 \mathrm{~V}, V_{R E F}=0.3 \mathrm{~V}$, sample rate $=60 \mathrm{KS} / \mathrm{s}$. 
power increases exponentially with an increasing $V_{D D}$. As $V_{D D}$ increases higher sample rates can be achieved, as the comparator can achieve a higher bandwidth and the analog switches have a lower ON resistance. Taking into account the sample rate of the ADC a plot of energy per cycle is shown in Figure 5.16c. Similar to the first ADC, operating this ADC in the sub-threshold region of operation provides the highest energy efficiency per conversion cycle. For this ADC, the input range is half the supply voltage, and although it is still possible to operate the ADC below $0.4 \mathrm{~V}$ the LSB becomes very small and the accuracy begins to degrade. Although this ADC has a smaller LSB than the first ADC it has the advantage of using a higher supply voltage since there are no on-chip voltages that go above or below the rail like the case in the first ADC. By using the higher supply voltage the ADC can operate at $4 \mathrm{MS} / \mathrm{s}$, while still achieving less than $18 \mathrm{pJ} /$ cycle. The optimum energy efficiency for the second ADC was determined to be $8.5 \mathrm{pJ} /$ cycle when $V_{D D}$ was $0.4 \mathrm{~V}$. Further decreasing of $V_{D D}$ significantly decreases the accuracy of this $\mathrm{ADC}$, as the input range is one half of the input range of the first ADC. The addition of the tunable capacitor was used to help overcome this degrade in accuracy due to a smaller input range.

\subsubsection{Temperature}

Temperature was swept from -30 degrees Celsius to 150 degrees Celsius and the full ADC ICGCUKAH was simulated. The input was set to $V_{R E F} / 2+$ analog GND where the output should be 01111111 . The output was correct for between -30 degrees Celsius to 80 degrees Celsius as shown in Figure 5.17a. Outside this temperature range the comparator fails to function properly and its offset voltage becomes much 


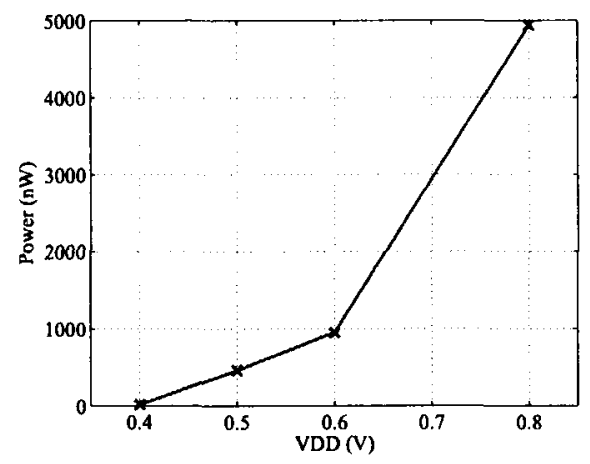

(a)

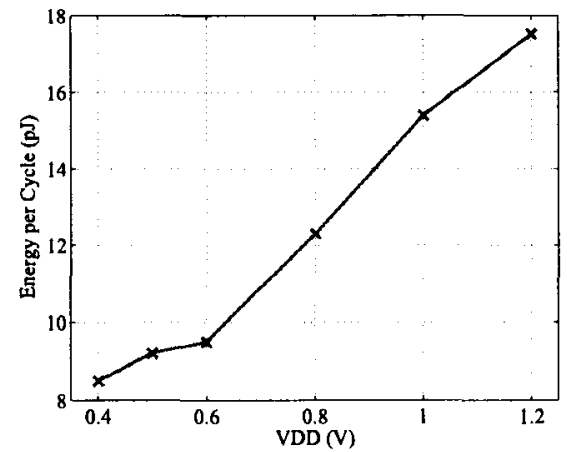

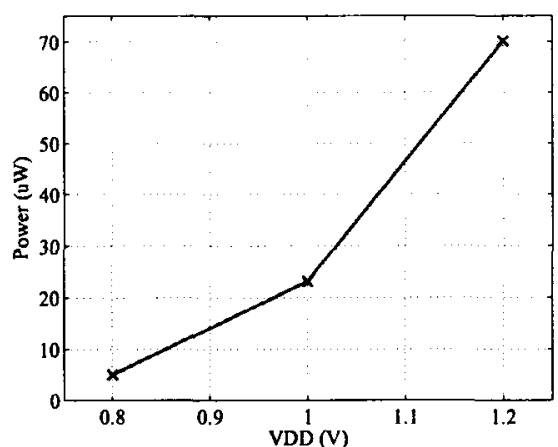

(b)

(c)

Figure 5.16: (a) Power dissipation of full ADC ICGCUKAH vs. $V_{D D}$. Sample rate $=20 \mathrm{kS} / \mathrm{s}, 40 \mathrm{kS} / \mathrm{s}, 100 \mathrm{kS} / \mathrm{s}, 400 \mathrm{kS} / \mathrm{s}$ for $V_{D D}$ of $0.4,0.5,0.6$ and $0.8 \mathrm{~V}$ respectively. (b) Power dissipation of full ADC ICGCUKAH vs. $V_{D D}$. Sample rate $=400 \mathrm{kS} / \mathrm{s}$, $1.5 \mathrm{MS} / \mathrm{s}, 4 \mathrm{MS} / \mathrm{s}$ for $V_{D D}$ of $0.8,1.0$ and $1.2 \mathrm{~V}$ respectively. (c) Energy per cycle of full ADC ICGCUKAH vs. $V_{D D}$. Sample rate $=20 \mathrm{kS} / \mathrm{s}, 40 \mathrm{kS} / \mathrm{s}, 100 \mathrm{kS} / \mathrm{s}, 400 \mathrm{kS} / \mathrm{s}$, $1.5 \mathrm{MS} / \mathrm{s}$ and $4 \mathrm{MS} / \mathrm{s}$ for $V_{D D}$ of $0.4,0.5,0.6,0.8,1.0$ and $1.2 \mathrm{~V}$ respectively. 


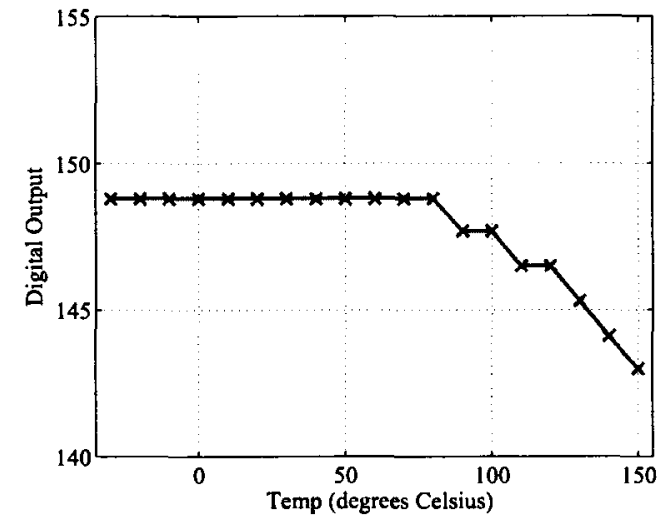

(a)

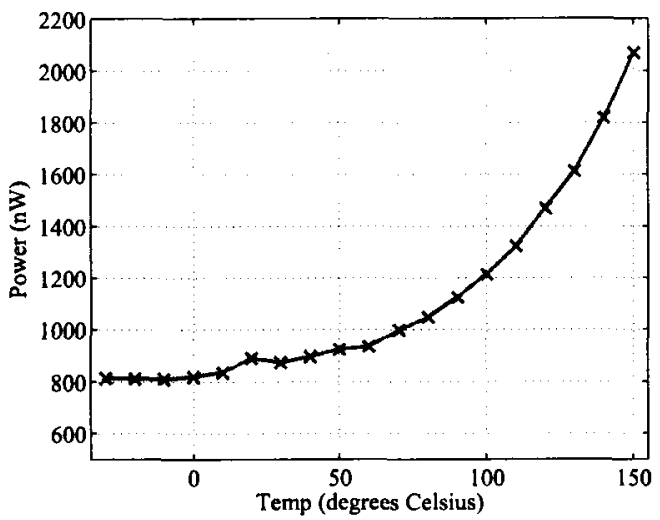

(b)

Figure 5.17: (a) Digital output of full ADC ICGCUKAH vs. temperature when analog input is set to $150 \mathrm{mV}, V_{D D}=0.6 \mathrm{~V}, V_{R E F}=0.3 \mathrm{~V}$, sample rate $=60 \mathrm{kS} / \mathrm{s}$. (b) Power dissipation of full ADC ICGCUKAH vs. temperature for $V_{D D}=0.6 \mathrm{~V}, V_{R E F}=0.3 \mathrm{~V}$, sample rate $=60 \mathrm{kS} / \mathrm{s}$.

more significant, leading to errors for the ADC output. It is possible to compensate for the offset voltage by readjusting the bias current, but this requires additional biasing circuitry and thus additional power dissipation. Similar to the case of the ADC ICGCUADC, the power dissipation with respect to temperature has an increasing exponential current relationship as shown in Figure 5.9b. The comparator being biased in sub-threshold increases in current as temperature is increased.

\subsubsection{Monte Carlo Results}

Monte Carlo statistical analysis was performed for the entire ADC, with an input of $V_{R E F} / 2$, and the output and power were examined. Process and mismatch variation were enabled for the models and no correlation or matching was assumed for the transistors allowing for a pessimistic analysis. The case was when the ADC had a supply voltage of $0.6 \mathrm{~V}$ (sample rate of $60 \mathrm{kS} / \mathrm{s}$ ) with the results shown in Figure 5.18. 

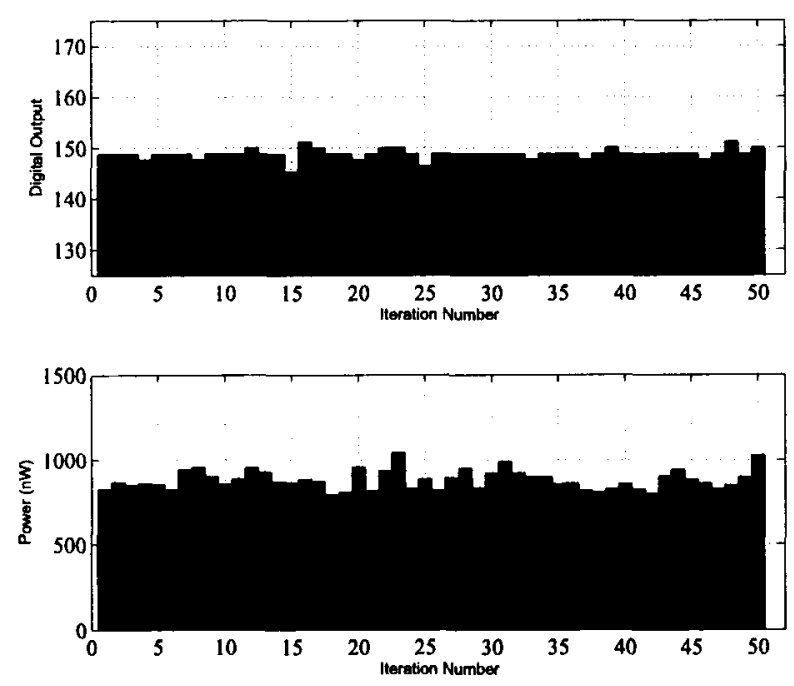

Figure 5.18: Output and Power dissipation of full ADC ICGCUKAH for 50 Monte Carlo iterations with process and mismatch for $V_{D D}=0.6 \mathrm{~V}, V_{I N}=0.15 \mathrm{~V}, V_{R E F}=0.3 \mathrm{~V}$, sample rate $=60 \mathrm{kS} / \mathrm{s}$. The output ranged from $145.3 \mathrm{mV}$ to $151.2 \mathrm{mV}$ with a mean, $\mu=148.8 \mathrm{mV}$ and $\sigma=0.96 \mathrm{mV}$. The power ranged from $792 \mathrm{nW}$ to $1043 \mathrm{nW}$ with a mean, $\mu=880.9 \mathrm{nW}$ and $\sigma=57.5 \mathrm{nW}$.

For the 50 iterations the output ranged from $145.3 \mathrm{mV}$ to $151.2 \mathrm{mV}$ with an average output of $148.8 \mathrm{mV}$ and a standard deviation of $0.96 \mathrm{mV}$ or 0.819 LSBs. The output was correct 34 times out of the 50 runs. For power dissipation, it ranged from $792 \mathrm{nW}$ to $1043 \mathrm{nW}$ with an average of $880.9 \mathrm{nW}$ and a standard deviation of $57.5 \mathrm{nW}$. The energy per conversion cycle is between $13.2 \mathrm{pJ} /$ sample and $17.4 \mathrm{pJ} /$ sample.

\subsection{Summary of Simulation Results}

The simulated results including parasitics based on the full layout for the first ADC, ICGCUADC in Table 5.1 and for the second ADC, ICGCUKAH in Table 5.2. The ADC ICGCUADC is more linear where the DNL is about half the DNL as the ADC, ICGCUKAH. For input range ICGCUADC has double the input range as 
ICGCUKAH allowing for a larger LSB and it relaxes the requirements of the comparator. The most energy efficient design appears to be ICGCUKAH, however this ADC requires an additional bias voltage to be used as an analog ground, which would likely be an additional band-gap reference voltage which would add additional power dissipation. The first ADC has the option of simply using $V_{D D}$ and $V_{S S}$ as $V_{R E F}$ and analog GND respectively.

Two main advantages of ICGCUKAH over ICGCUADC is that firstly it has the option of tuning the attenuation capacitor instead of having a fixed capacitance. Although ICGCUADC appears to be more linear than ICGCUKAH this assumes no process and mismatch variation. Thus, the tuning should allow to achieve an optimum linearity and dynamic performance after process and mismatch variation are added. The second main advantage is that it can achieve a higher speed, as a higher supply voltage can be used. A $V_{D D}$ of $1.2 \mathrm{~V}$ allows this $\mathrm{ADC}$ to sample at speeds higher than $4 \mathrm{MS} / \mathrm{s}$. Both designs have similar temperature ranges where ICGCUADC works 20 degrees Celsius higher than ICGCUKAH but ICGCUKAH works 20 degrees Celsius lower than ICGCUADC. Layout area is the same for both chips, as the tunable capacitor for ICGCUKAH and the top capacitor switches for ICGCUADC use approximately the same amount of chip area.

\subsubsection{Application Example}

A recorded ECG signal was input into the first ADC as shown in Figure 5.19 and sampled at $1 \mathrm{kS} / \mathrm{s}$ with a $V_{D D}$ of $0.4 \mathrm{~V}$. The digitized output is shown in Figure 5.19. The average power dissipation was $85.9 \mathrm{nW}$ over the entire simulation. A recorded 
Table 5.1: Summary of Simulated Results for ICGCUADC

\begin{tabular}{|c|c|c|c|}
\hline$V_{D D}$ & $\mathbf{0 . 4} \mathbf{~ V}$ & $\mathbf{0 . 6} \mathrm{V}$ & $\mathbf{0 . 8} \mathrm{V}$ \\
\hline Input Range & $0.4 \mathrm{~V}$ & $0.6 \mathrm{~V}$ & $0.8 \mathrm{~V}$ \\
\hline Sample Rate & $20 \mathrm{kS} / \mathrm{s}$ & $60 \mathrm{kS} / \mathrm{s}$ & $150 \mathrm{kS} / \mathrm{s}$ \\
\hline INL & & $0.26 \mathrm{LSB}$ & \\
\hline DNL & & $0.35 \mathrm{LSB}$ & \\
\hline Power & $213 \mathrm{nW}$ & $937 \mathrm{nW}$ & $3.64 \mu \mathrm{W}$ \\
\hline SNDR $^{*}$ & $49.8 \mathrm{~dB}$ & $49.9 \mathrm{~dB}$ & $50.6 \mathrm{~dB}$ \\
\hline ENOB* $^{*}$ & 7.97 & 7.99 & 8.11 \\
\hline SFDR $^{*}$ & $60.8 \mathrm{~dB}$ & $62.2 \mathrm{~dB}$ & $63.6 \mathrm{~dB}$ \\
\hline Energy per Sample & $10.7 \mathrm{pJ}$ & $15.6 \mathrm{pJ}$ & $24.2 \mathrm{pJ}$ \\
\hline Monte Carlo** & $37 / 50 \mathrm{correct}$ & $39 / 50$ correct & $41 / 50$ correct \\
\hline Temperature Range & & $-10^{\circ} \mathrm{C}$ to $100^{\circ} \mathrm{C}$ & \\
\hline Area & & $0.125 \mathrm{~mm}$ & \\
\hline Technology & & $0.13 \mu \mathrm{m} \mathrm{CMOS}$ & \\
\hline
\end{tabular}

* SR=60.3kS $/ \mathrm{s} V_{D D}=0.6 f_{I N}=600 \mathrm{~Hz}, \mathrm{SR}=10 \mathrm{kS} / \mathrm{s} V_{D D}=0.4 f_{I N}=100 \mathrm{~Hz}$ and $\mathrm{SR}=250 \mathrm{kS} / \mathrm{s} V_{D D}=0.8 f_{I N}=1500 \mathrm{~Hz}$

** Process and mismatch variation with no matching, correct output out of 50 iterations.

Table 5.2: Summary of Simulated Results for ICGCUKAH

\begin{tabular}{|c|c|c|c|}
\hline$V_{D D}$ & $0.4 \mathrm{~V}$ & $\mathbf{0 . 6} \mathrm{V}$ & $\mathbf{0 . 8} \mathrm{V}$ \\
\hline Input Range & $0.2 \mathrm{~V}$ & $0.3 \mathrm{~V}$ & $0.4 \mathrm{~V}$ \\
\hline Sample Rate & $20 \mathrm{kS} / \mathrm{s}$ & $100 \mathrm{kS} / \mathrm{s}$ & $400 \mathrm{kS} / \mathrm{s}$ \\
\hline INL & & $0.38 \mathrm{LSB}$ & \\
\hline DNL & & $0.83 \mathrm{LSB}$ & \\
\hline Power & $170 \mathrm{nW}$ & $950 \mathrm{nW}$ & $4.94 \mu \mathrm{W}$ \\
\hline SNDR $^{*}$ & & $49.9 \mathrm{~dB}$ & \\
\hline ENOB* $^{*}$ & & 7.99 & \\
\hline SFDR $^{*}$ & & $56.2 \mathrm{~dB}$ & \\
\hline Energy per Sample & $8.5 \mathrm{pJ}$ & $9.5 \mathrm{pJ}$ & $12.3 \mathrm{pJ}$ \\
\hline Monte Carlo** & & $34 / 50$ & \\
\hline Temperature Range & & $-30^{\circ} \mathrm{C}$ to $80^{\circ} \mathrm{C}$ & \\
\hline Area & & $0.125 \mathrm{~mm}^{2}$ & \\
\hline Technology & & $0.13 \mu \mathrm{m} \mathrm{CMOS}$ & \\
\hline
\end{tabular}

* $\mathrm{SR}=60.3 \mathrm{kS} / \mathrm{s} V_{D D}=0.6 f_{I N}=1000 \mathrm{~Hz}$

** Process and mismatch variation with no matching, correct output out of 50 iterations. 


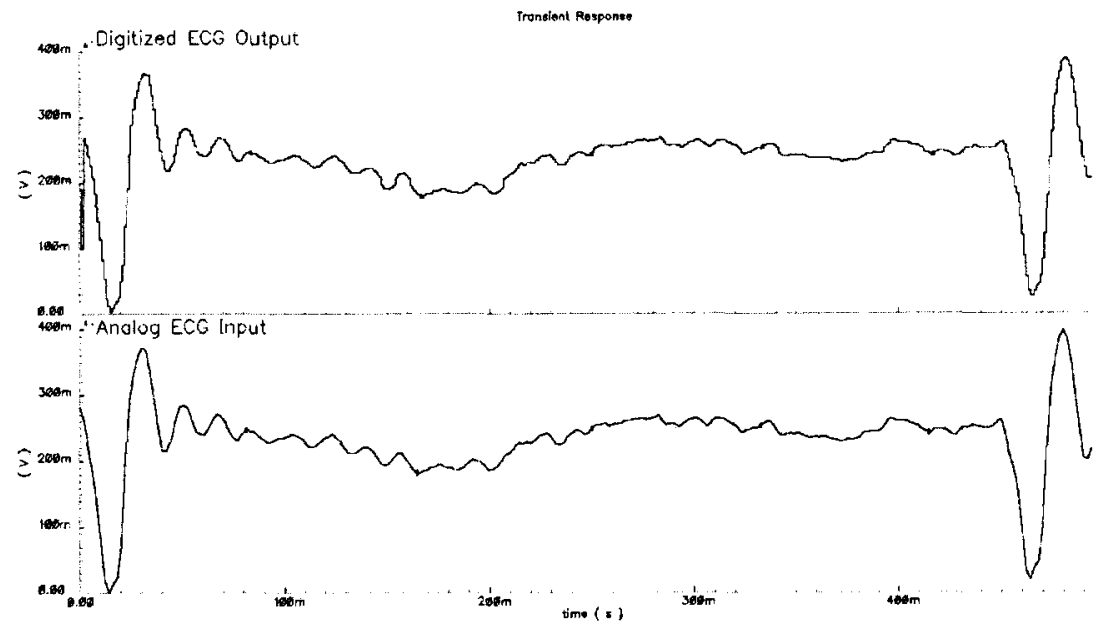

Figure 5.19: An ECG signal digitized when supply voltage is $0.4 \mathrm{~V}$ at a sample rate of $1 \mathrm{kS} / \mathrm{s}$ and a $V_{R E F}$ of $0.4 \mathrm{~V}$ for ICGCUADC is shown for visual comparison.

ECG signal was input into the second ADC as shown in Figure 5.20 and sampled at $1 \mathrm{kS} / \mathrm{s}$ with a $V_{D D}$ of $0.4 \mathrm{~V}$. The digitized output is shown in Figure 5.20. The average power dissipation was $82.8 \mathrm{nW}$ over the entire simulation. 


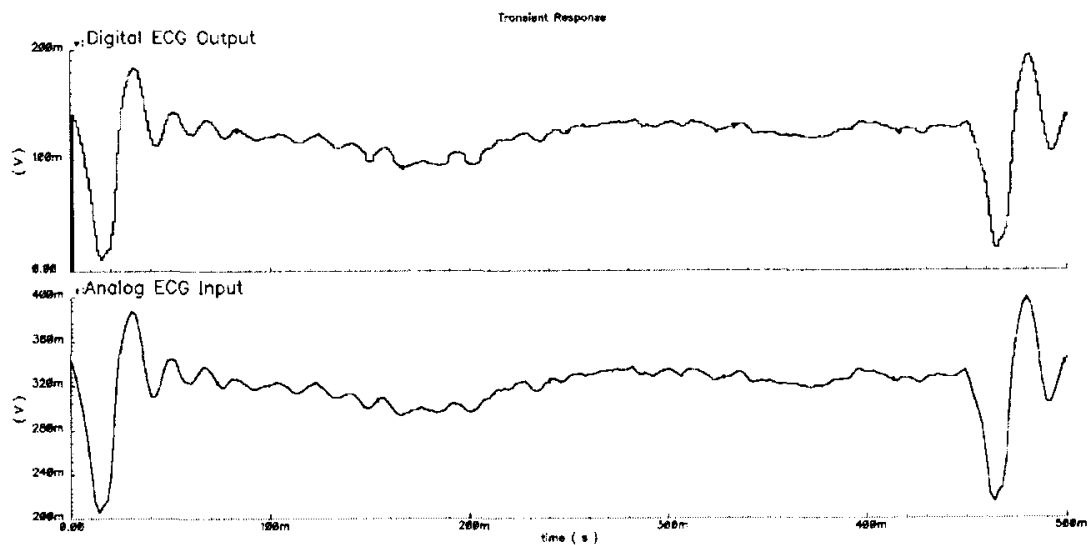

Figure 5.20: An ECG signal digitized when supply voltage is $0.4 \mathrm{~V}$ at a sample rate of $1 \mathrm{kS} / \mathrm{s}$ and a $V_{R E F}$ of $0.2 \mathrm{~V}$ for ICGCUKAH is shown for visual comparison. 


\section{Chapter 6}

\section{Measurements}

\subsection{Measured Results of Analog to Digital Con- verter 1}

A photograph of the first ADC chip, ICGCUADC, is shown in Figure 6.1a. The test setup is shown in Figure 6.1b. The chip was bonded to a PCB using aluminum bond wires and a wedge bonder, with the bond wires shown in a scanning electron microscope photograph in Figure 6.2. The PCB contained decoupling capacitors for the DC pins such as $V_{D D}$ (analog, digital and buffer), $V_{R E F}, I_{\text {bias }}$, and $V_{\text {tune. The }}$ Analog IN and digital clk pins were connected through standard SMA connectors on the PCB. The pins to reset the digital logic and to select offset correction was done through a DIP switch on the PCB. The PCB also contained ESD protection and zener diodes for the supply voltages on chip, with leakage currents being below $20 \mathrm{nA}$ for these components.

\subsubsection{Linearity Results: INL and DNL}

Static measurements for the first ADC chip were conducted by applying a DC signal into the input of ADC and adjusting its value until the LSB was switching with 


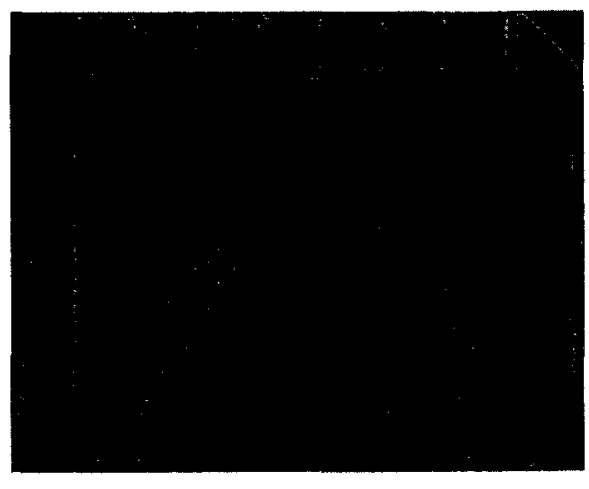

(a)

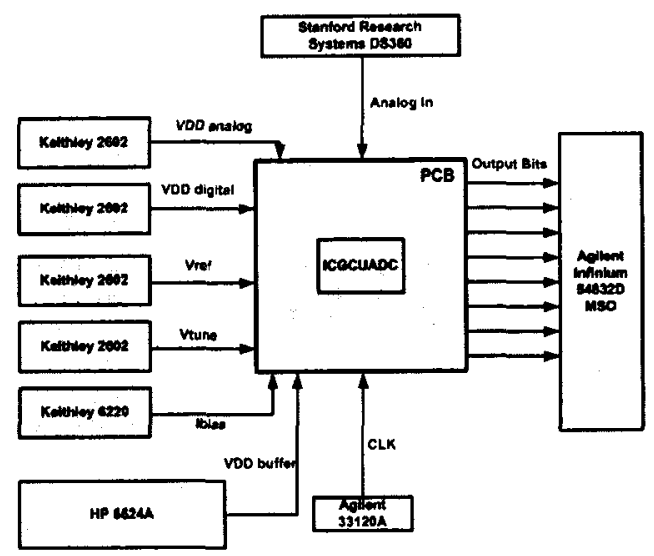

(b)

Figure 6.1: (a) Photograph of ADC ICGCUADC. (b) Test setup of ICGCUADC.

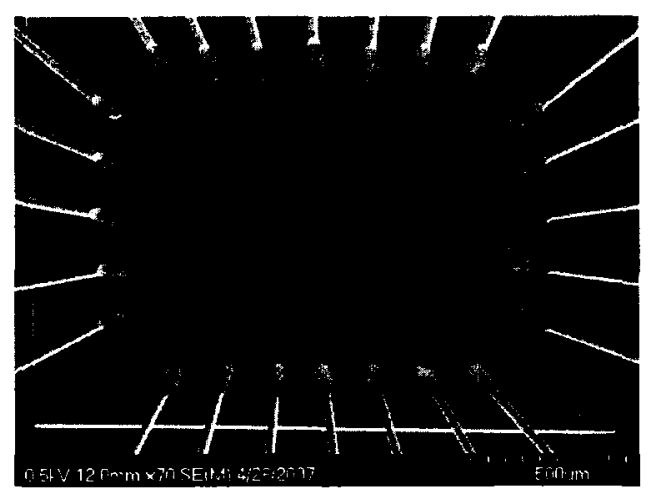

(a)

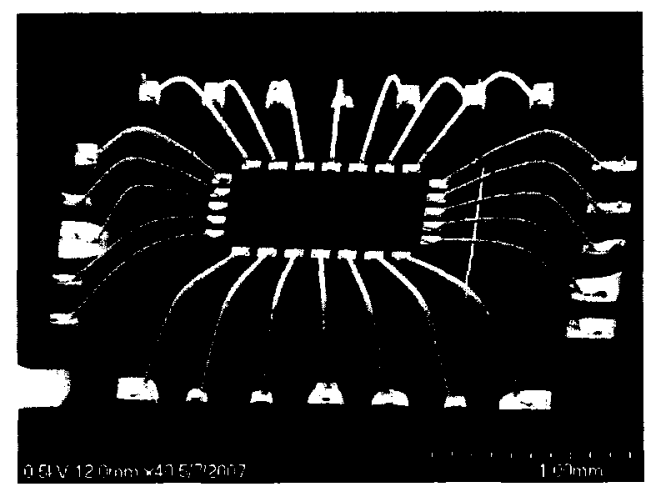

(b)

Figure 6.2: Scanning electron microscope photograph of the bond wires that connect the PCB to the chip. 


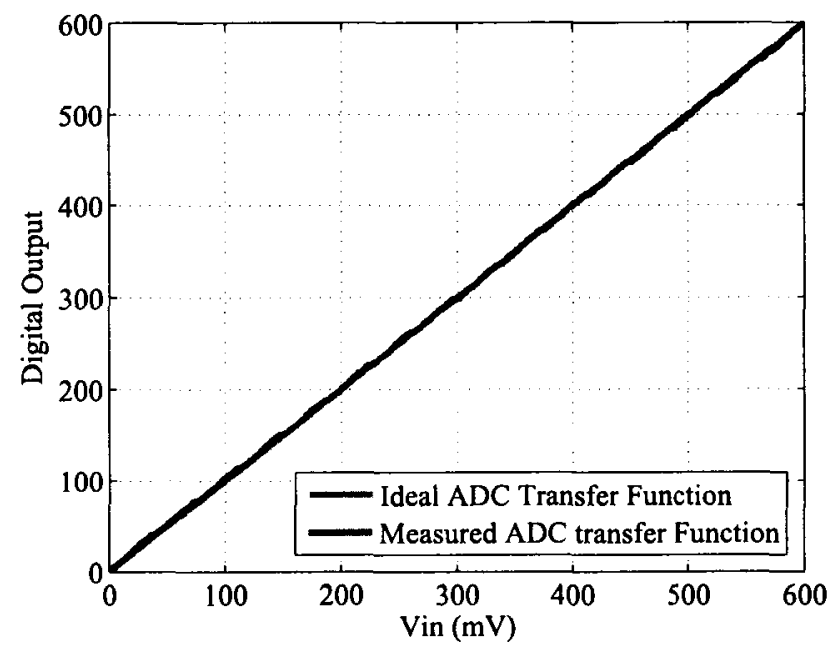

Figure 6.3: Measured digital output versus analog input for ADC ICGCUADC at $60 \mathrm{kS} / \mathrm{s}$ with $\mathrm{Vref}=0.6$ and $V_{D D}=0.6 \mathrm{~V}$.

an approximately 50 percent duty cycle. The switch points were plotted with its corresponding digital output in the transfer function shown in Figure 6.3. The lighter line in Figure 6.3 shows a transfer function of an ideal 8-bit ADC. There were 2 missing codes for the ADC, the case when the output is 01111111 and when the output is 00111111 . Also when the input causes the ADC output to be $\operatorname{xxxx1110}$ and is further increased to transition to $\mathrm{xxxx} 1111$, this transition occurs very quickly and results in switching to $\mathrm{xxx} 10000$ faster than expected. The DNL was found to be -1 LSB because of the missing code and the INL was found to be 1.05 LSB as shown in Figure $6.4 \mathrm{a}$ and $6.4 \mathrm{~b}$ respectively. The attenuation capacitor within the capacitor array is likely not to have the exact value of capacitance required for the capacitor array, which would lead to every 16 th bit (four capacitors on each side of capacitor array) having a larger DNL than expected. 


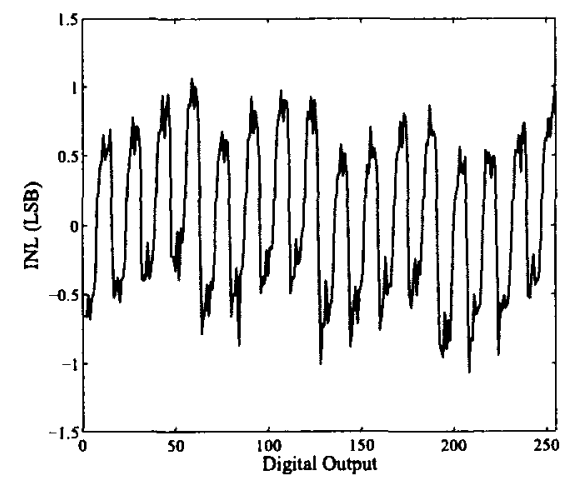

(a)

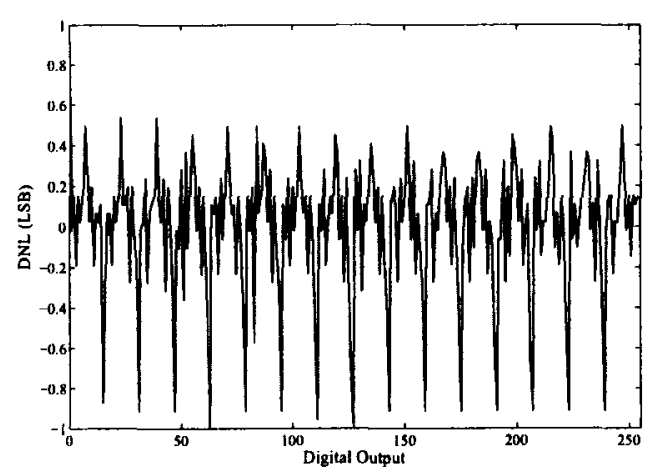

(b)

Figure 6.4: (a) Measured INL plot for ADC ICGCUADC with a sample rate of $60 \mathrm{kS} / \mathrm{s}$, Vref $=0.6$ and $V_{D D}=0.6 \mathrm{~V}$. (b) Measured DNL plot for ADC ICGCUADC with a sample rate of $60 \mathrm{kS} / \mathrm{s}, \mathrm{Vref}=0.6$ and $V_{D D}=0.6 \mathrm{~V}$.

\subsubsection{Dynamic Results: SFDR, SINAD and ENOB}

For dynamic testing a sinusoid input from $0 \mathrm{~V}$ to $V_{R E F}$ was input into the ADC at some input frequency below the Nyquist frequency. In this example a $0.6 \mathrm{~V}$ pk-pk sinusoid at $600 \mathrm{~Hz}$ was applied to the input of the ADC. Next, the DFT was taken of the digitized output from the ADC with the result shown in Figure 6.5. This process was repeated for many different input frequencies and different ADC supply voltages, at which the SINAD, ENOB and SFDR can be extracted from the DFTs for each input frequency. It is important to have a "pure" sinusoid signal with little distortion so that the quantization noise and distortion in the DFT is from the ADC being tested and not a result of a distorted input signal. The Stanford Research Systems DS360 is an ultra-low distortion source that was used to input the sinusoid signals to the ADC.

A plot of measured SINAD and SFDR vs. input frequency is shown in Figure 
6.6a for the case when the supply voltage is $0.4 \mathrm{~V}$, in Figure $6.6 \mathrm{~b}$ for the case when the supply voltage is $0.6 \mathrm{~V}$, and in Figure $6.6 \mathrm{c}$ when the supply is $0.8 \mathrm{~V}$.

The ADC achieves above 40dB SINAD for frequencies below $400 \mathrm{~Hz}$ and above $40 \mathrm{~dB}$ SFDR for frequencies above $1 \mathrm{KHz}$ when sampled at $10 \mathrm{kS} / \mathrm{s}$ with a $V_{D D}$ of $0.4 \mathrm{~V}$. As the input frequency approaches Nyquist frequency, the SINAD and SFDR begin to decrease close to approximately $20 \mathrm{~dB}$. For the case of $0.6 \mathrm{~V} V_{D D}$, the ADC achieves above $40 \mathrm{~dB}$ for frequencies below $4 \mathrm{kHz}$ and a SFDR above $40 \mathrm{~dB}$ for frequencies below $12 \mathrm{kHz}$ when sampled at $60 \mathrm{kS} / \mathrm{s}$, with the SINAD decreasing to $25 \mathrm{~dB}$ as the input frequency approaches the Nyquist frequency. The last case with a $V_{D D}$ of $0.8 \mathrm{~V}$, the ADC achieves above $40 \mathrm{~dB}$ for frequencies below $4 \mathrm{kHz}$ and a SFDR above $40 \mathrm{~dB}$ for frequencies below $40 \mathrm{kHz}$ when sampled at $100 \mathrm{kS} / \mathrm{s}$, with the SINAD decreasing to approximately $30 \mathrm{~dB}$ as the input frequency approaches the Nyquist frequency. The decreasing SINAD and SFDR with increasing input frequency was expected as simulated in the last section. The bandwidth of the switches, especially at lower supply voltages, as well as differences in delay in the digital control signals which can cause significant time between the sampling clock to when the input signal is held for conversion adds additional noise to the ADC output, especially at higher input frequencies.

All three cases are shown in Figure 6.7 with the input frequency normalized by dividing it by the sample rate. The higher $V_{D D}$ allows for a less decreasing SINAD and SFDR as the Nyquist frequency is approached. This is very similar to the simulated case as shown in Figure 5.4. The switches in this case are more linear, than when operating in full sub-threshold $(0.4 \mathrm{~V}$ or lower $)$, the LSB is higher $\left(V_{D D} / 256\right)$ which 


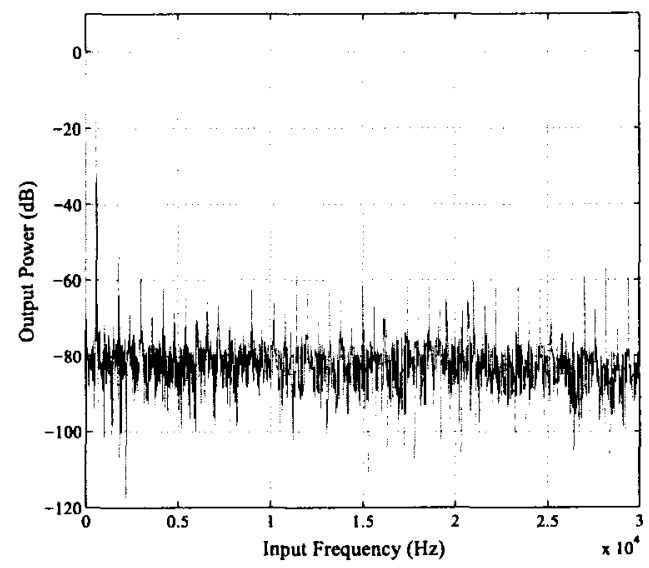

Figure 6.5: DFT of measured output of ADC ICGCUADC, when input is a $600 \mathrm{kHz}$, $0.6 \mathrm{~V}$ pk-pk Sinusoid. For the ADC, $V_{D D}=V_{R E F}=0.6 \mathrm{~V}$ and sample rate $=60 \mathrm{kS} / \mathrm{s}$.

relaxes the effect of noise and offset of the comparator. The capacitor mismatch of the ADC led to a maximum obtainable SINAD of approximately $43 \mathrm{~dB}$ for each value of $V_{D D}$ selected.

Similarly, the ENOB can be calculated from the SINAD for each $V_{D D}$ as is shown in Figure 6.8. All three cases have an ENOB of between 6.5 and 7.01 bits at low frequencies and decrease as the input frequency is increasing, similarly to the SINAD.

\subsubsection{Power Dissipation}

As shown in Figure 6.9a the simulated and measured power dissipation for different supply voltages are in close agreement. The optimum energy efficiency was determined to be $10 \mathrm{pJ} /$ cycle when $V_{D D}$ was $0.4 \mathrm{~V}$ which is a slight improvement on the $11 \mathrm{pJ} /$ cycle seen during simulation*, as shown in Figure 6.9b. A further decreasing of

\footnotetext{
*Although within the simualted Monte Carlo range.
} 


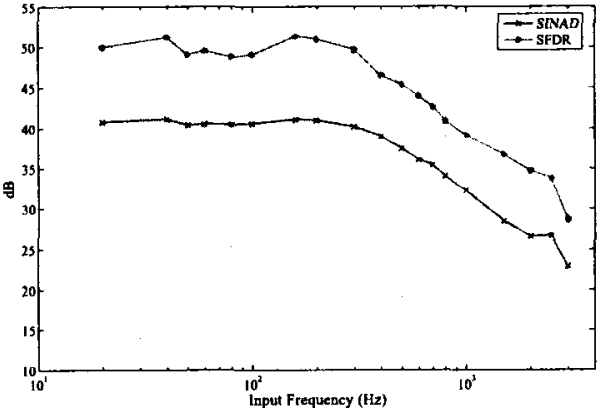

(a)

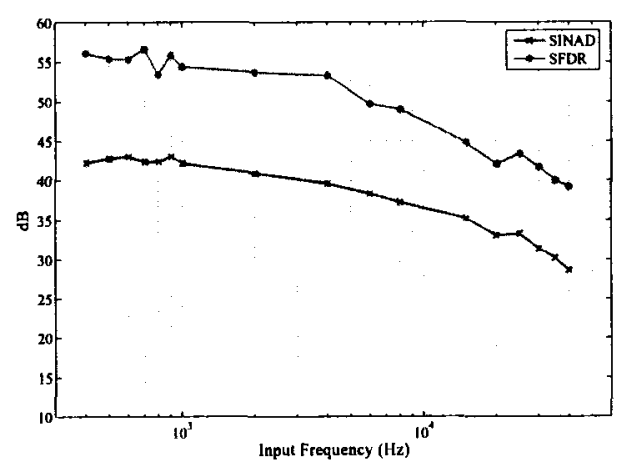

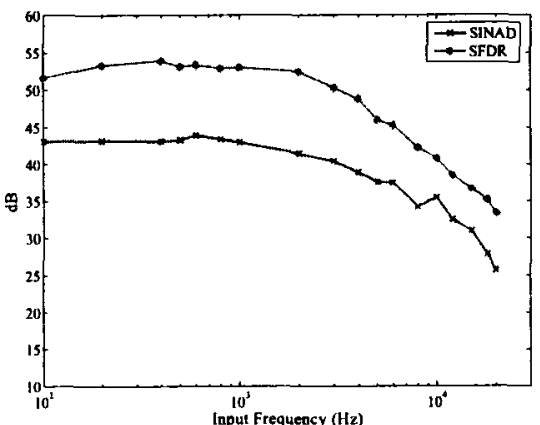

(b)

(c)

Figure 6.6: (a) Measured Signal-to-Noise and Distortion (SINAD) and Spurious Free Dynamic Range (SFDR) vs. input frequency when $V_{D D}=V_{R E F}=0.4 \mathrm{~V}$, and a sample rate of $10 \mathrm{kS} / \mathrm{s}$. (b) Measured Signal-to-Noise and Distortion (SINAD) and Spurious Free Dynamic Range (SFDR) vs. input frequency when $V_{D D}=V_{R E F}=0.6 \mathrm{~V}$, and a sample rate of $60 \mathrm{kS} / \mathrm{s}$. (c) Measured Signal-to-Noise and Distortion (SINAD) and Spurious Free Dynamic Range (SFDR) vs. input frequency when $V_{D D}=V_{R E F}=0.6 \mathrm{~V}$, and a sample rate of $60 \mathrm{kS} / \mathrm{s}$. 


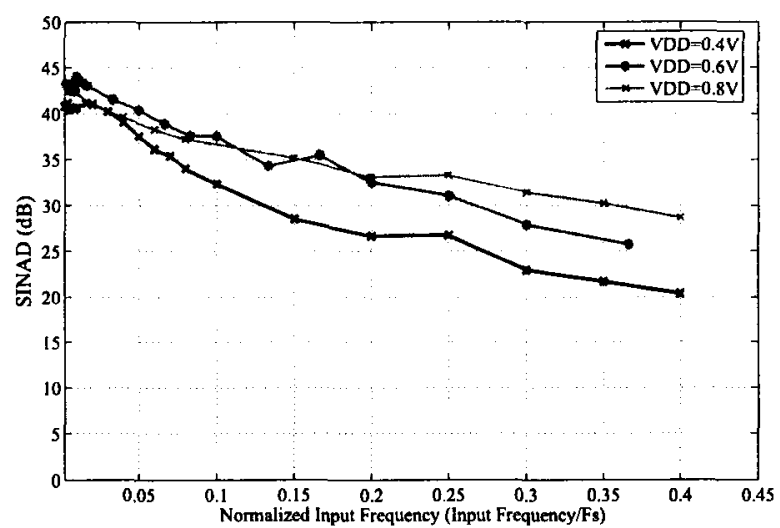

Figure 6.7: Measured Signal-to-Noise and Distortion (SINAD) vs. normalized frequency when $V_{D D}=V_{R E F}=0.8 \mathrm{~V}, 0.6 \mathrm{~V}, 0.4 \mathrm{~V}$

$V_{D D}(0.3 \mathrm{~V}$ or below $)$ significantly decreases the bandwidth which agrees with the simulated results. The optimum $V_{D D}$ for this ADC in terms of energy efficiency should be between 0.3 and $0.6 \mathrm{~V}$. Further increasing of $V_{D D}$ results in many of the transistors operating in saturation region and more energy is required per conversion cycle. Shown in Figure $6.9 \mathrm{a}$ the $V_{D D}$ is kept at $0.4 \mathrm{~V}$ and the sample rate is increased between approximately $1 \mathrm{kS} / \mathrm{S}$ and $20 \mathrm{kS} / \mathrm{s}$ with the majority of power being used by the analog components. The analog circuitry, digital circuitry, and capacitor array $\left(V_{R E F}\right)$ all showed a linear increase in power dissipation as the sampled rate is increased. Similarly, shown in Figure $6.9 \mathrm{~b}$ the $V_{D D}$ is kept at $0.8 \mathrm{~V}$ and the sample rate is increased between approximately $50 \mathrm{kS} / \mathrm{S}$ and $425 \mathrm{kS} / \mathrm{s}$ with the majority of power again being used by the analog circuitry. Figure 6.11 shows measured analog circuitry power dissipation, digital circuitry power dissipation and capacitor array $\left(V_{R E F}\right)$ power dissipation all compared to the simulated power dissipation for different sample rates and a $V_{D D}$ of $0.6 \mathrm{~V}$. The measured analog circuitry power dissipation closely matches 


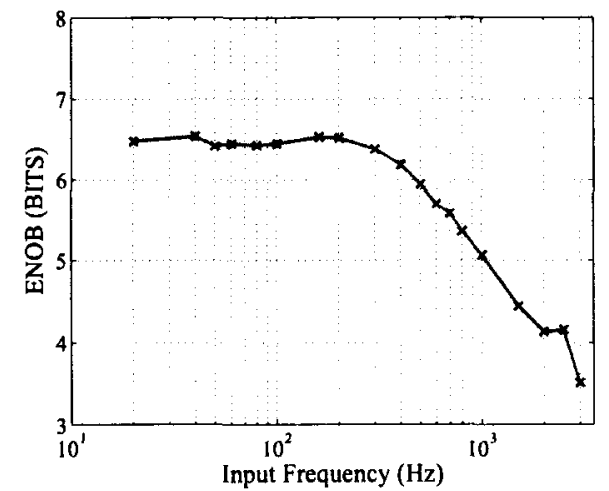

(a)

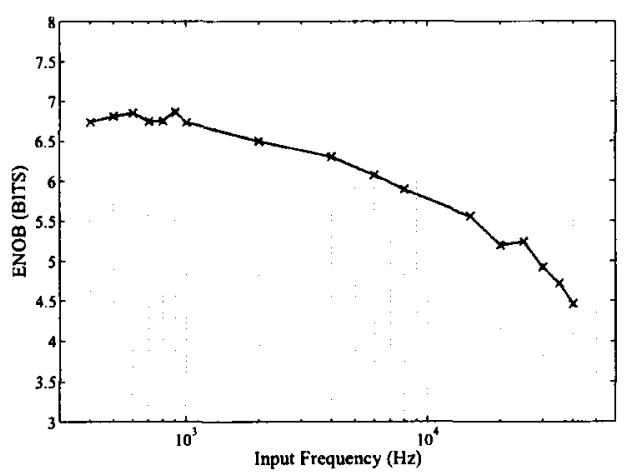

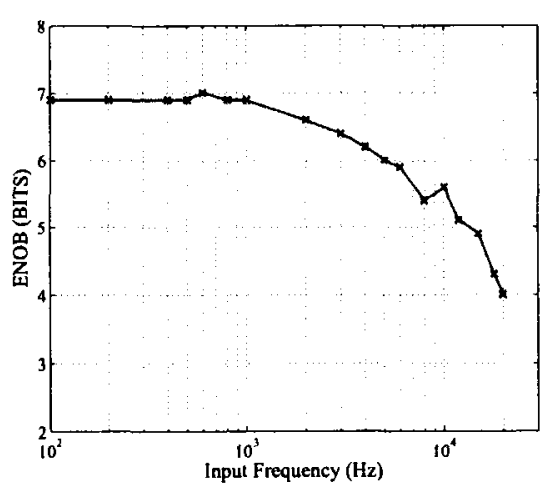

(b)

(c)

Figure 6.8: (a) Measured Effective Number of Bits (ENOB) vs. input frequency when $V_{D D}=V_{R E F}=0.4 \mathrm{~V}$, and a sample rate of $10 \mathrm{kS} / \mathrm{s}$. (b) Measured Effective Number of Bits (ENOB) and Spurious Free Dynamic Range (SFDR) vs. input frequency when $V_{D D}=V_{R E F}=0.6 \mathrm{~V}$, and a sample rate of $60 \mathrm{kS} / \mathrm{s}$. (c) Measured Effective Number of Bits (ENOB)) and Spurious Free Dynamic Range (SFDR) vs. input frequency when $V_{D D}=V_{R E F}=0.8 \mathrm{~V}$, and a sample rate of $100 \mathrm{kS} / \mathrm{s}$. 


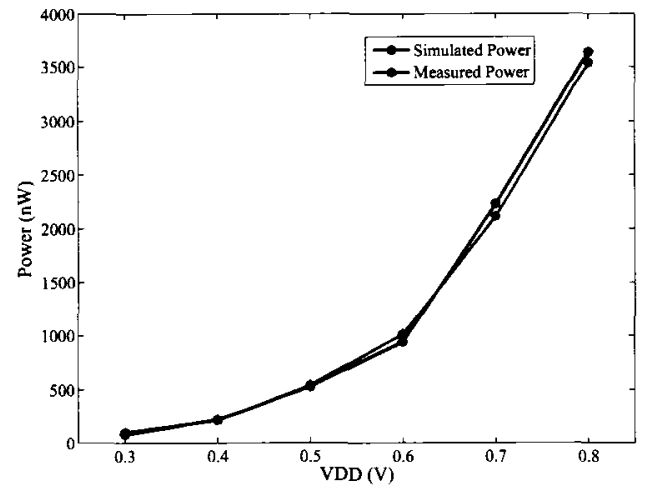

(a)

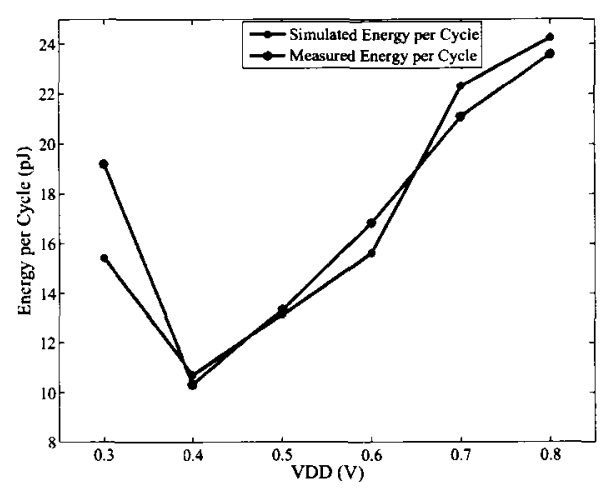

(b)

Figure 6.9: (a) Measured power dissipation of ADC ICGCUADC vs. $V_{D D}$. Sample rate $=5 \mathrm{kS} / \mathrm{s}, 20 \mathrm{kS} / \mathrm{s}, 40 \mathrm{kS} / \mathrm{s}, 60 \mathrm{kS} / \mathrm{s}, 100 \mathrm{kS} / \mathrm{s}$ and $150 \mathrm{kS} / \mathrm{s}$ for $V_{D D}$ of $0.3,0.4,0.5,0.6,0.7$ and $0.8 \mathrm{~V}$ respectively. (b) Measured energy per cycle of ADC ICGCUADC vs. $V_{D D}$. Sample rate $=5 \mathrm{kS} / \mathrm{s}, 20 \mathrm{kS} / \mathrm{s}, 40 \mathrm{kS} / \mathrm{s}, 60 \mathrm{kS} / \mathrm{s}, 100 \mathrm{kS} / \mathrm{s}$ and $150 \mathrm{kS} / \mathrm{s}$ for $V_{D D}$ of $0.3,0.4,0.5,0.6,0.7$ and $0.8 \mathrm{~V}$ respectively.

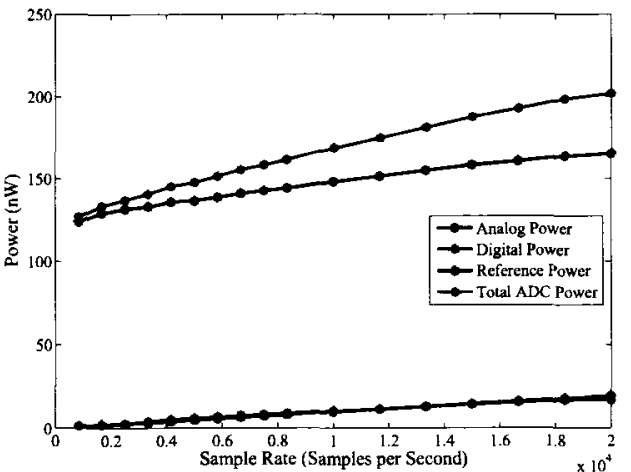

(a)

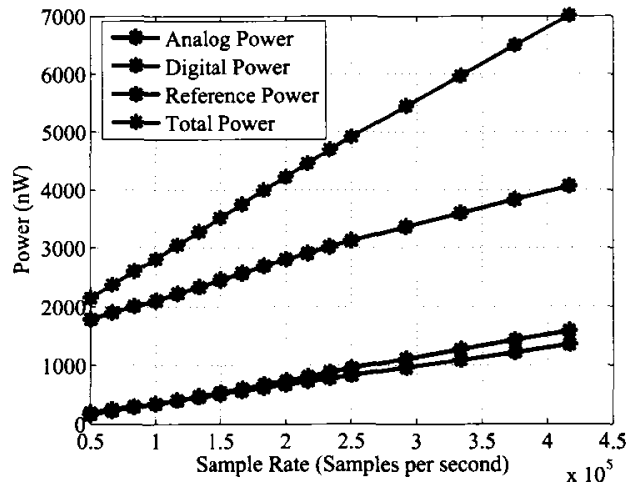

(b)

Figure 6.10: (a) Measured power dissipation of ADC ICGCUADC vs. sample rate for $V_{D D}=0.4 \mathrm{~V}$. (b) Measured power dissipation of ADC ICGCUADC vs. sample rate for $V_{D D}=0.8 \mathrm{~V}$. 
the simulated power dissipation. The digital circuitry power dissipation and capacitor array $\left(V_{R E F}\right)$ power dissipation were slightly higher for the measured chip than seen in simulations. However the largest difference in power dissipation is only $140 \mathrm{nW}$ vs. $185 \mathrm{nW}$ for the digital circuitry and $140 \mathrm{nW}$ vs. $170 \mathrm{nW}$ for the capacitor array $\left(V_{R E F}\right)$, which is less than $50 \mathrm{nW}$ difference for both. The analog circuitry also dissipates much more power than the digital circuitry and capacitor array $\left(V_{R E F}\right)$ which will lead to the measured ADC power dissipation closely matching the simulated ADC power dissipation. This is shown in Figure 6.12 for different sample rates, with the largest difference in power being approximately $100 \mathrm{nW}$ between measured and simulated.

\subsubsection{Offset Correction}

Although, the offset correction could not be tested in isolation because there was no external offset control signals for the comparator, it could be tested within the full ADC system as the $V_{\text {tune }}$ pin is directly connected to comparator node opposite the capacitor array (analog GND). By increasing or decreasing this voltage, the offset of the comparator can be set manually. A square wave was input between $0 \mathrm{~V}$ and $V_{R E F}$ $(0.6 \mathrm{~V})$, such that all bits will be switching between 00000000 and 11111111 . First, $V_{\text {tune }}$ is set to $-10 \mathrm{mV}$. Figure $6.13 \mathrm{a}$ shows the ADC output without offset correction and Figure $6.13 \mathrm{~b}$ with offset correction. The 2nd LSB bit is corrupted more without offset correction than with offset correction. Similarly when $V_{\text {tune }}$ is set to $10 \mathrm{mV}$, as shown in Figure 6.14a the case without offset correction has the third LSB bit corrupted, while the case with offset correction has no bits corrupted as shown in Figure 6.14b. The offset correction circuitry allows the ADC to function with an 


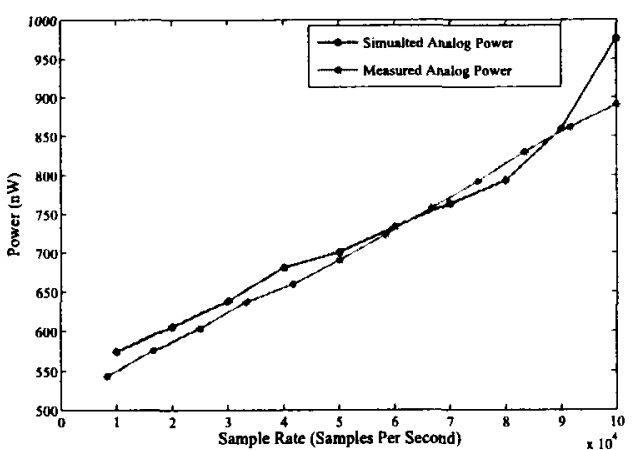

(a)

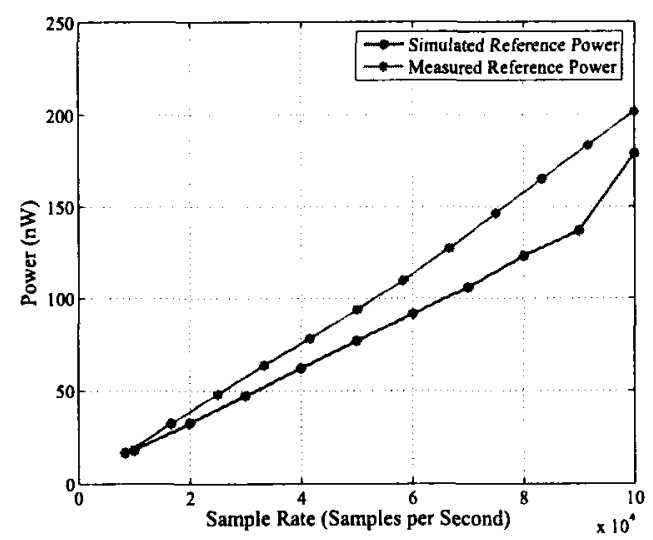

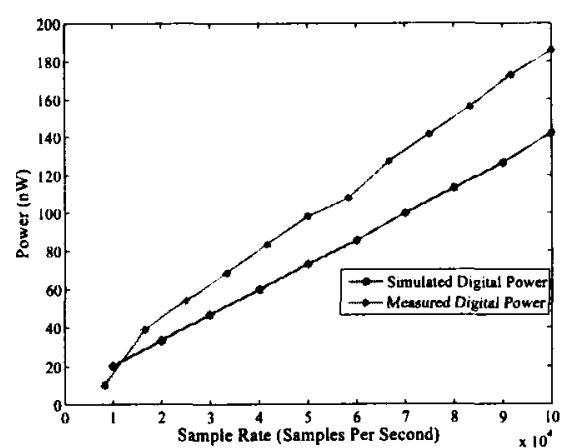

(b)

(c)

Figure 6.11: (a) Measured analog power dissipation vs. sample rate for $V_{D D}=0.6 \mathrm{~V}$. (b) Measured digital power dissipation vs. sample rate for $V_{D D}=0.6 \mathrm{~V}$. (c) Measured reference voltage power dissipation vs. sample rate for $V_{D D}=0.6 \mathrm{~V}$.

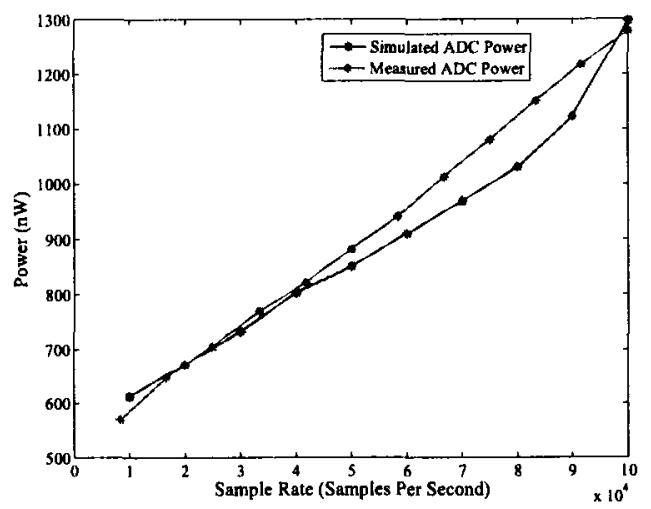

Figure 6.12: Measured Power dissipation of full ADC ICGCUADC vs. sample rate for $V_{D D}=0.6 \mathrm{~V}$ 


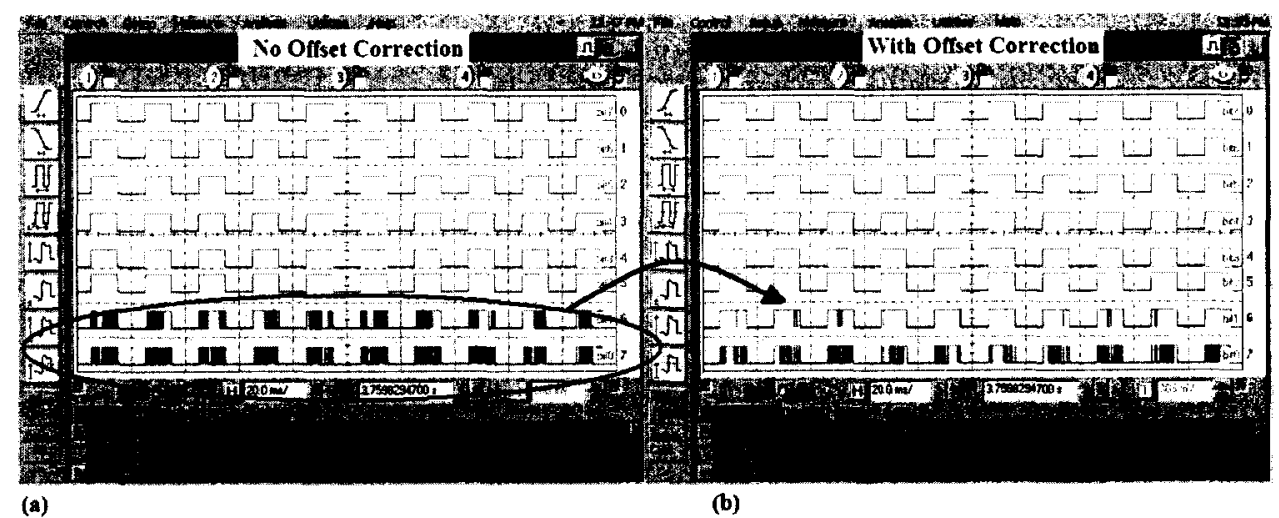

Figure 6.13: (a) Offset of $-10 \mathrm{mV}$ input into ADC comparator with offset correction turned off, when input is a square wave between $0 \mathrm{~V}$ and $V_{D D} . V_{D D}=V_{R E F}=0.6 \mathrm{~V}$, sample rate $=60 \mathrm{kS} / \mathrm{s}$. (b) Offset of $-10 \mathrm{mV}$ input into $\mathrm{ADC}$ comparator with offset correction turned on, when input is a square wave between $0 \mathrm{~V}$ and $V_{D D} . V_{D D}=V_{R E F}=0.6 \mathrm{~V}$, sample rate $=60 \mathrm{kS} / \mathrm{s}$.

input-offset between $-8 \mathrm{mV}$ and $25 \mathrm{mV}$, while without offset correction circuitry the ADC functions with an input-offset between $-3 \mathrm{mV}$ and $+3 \mathrm{mV}$ demonstrating the offset correction circuitry is working on-chip.

Another advantage of the offset correction is that it makes the comparator's accuracy less sensitive to the bias current. When offset correction is disabled, the bias current needs to be some constant value in order to keep the offset voltage minimized, and this changes as the supply voltage changes. When offset correction is enabled there is less importance to find the right bias current for the comparator. As long as the bandwidth for the comparator is adequate, the offset correction circuitry will ensure the offset voltage is minimized.

\subsubsection{Application Example}

An ECG signal was input from the Agilent source into the ADC sampled at $10 \mathrm{kS} / \mathrm{s}$ with a $V_{D D}$ of $0.4 \mathrm{~V}$ and as shown in Figure 6.15 the analog ECG input and the 


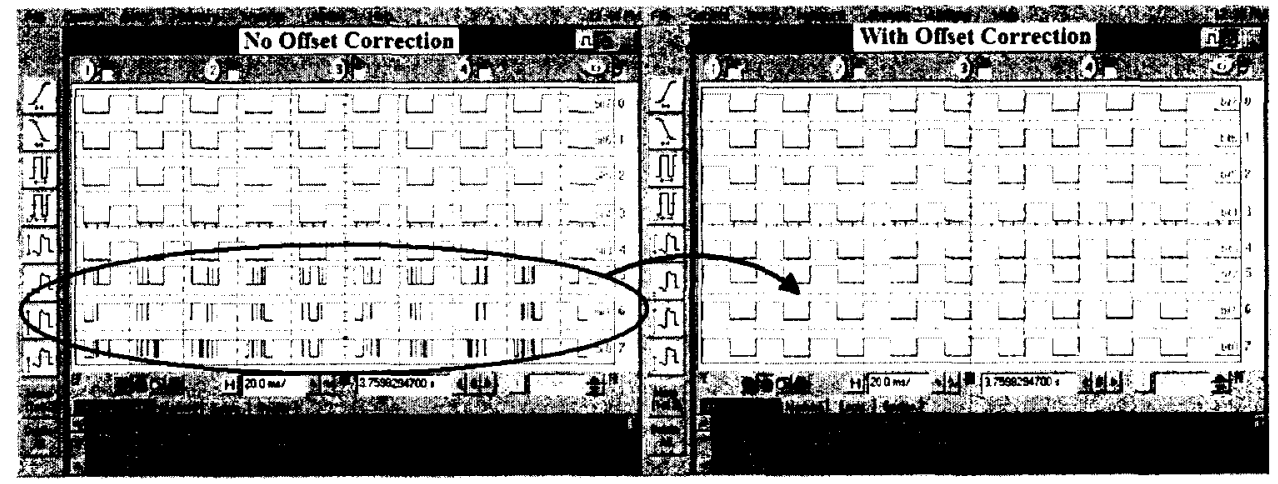

(a)

(b)

Figure 6.14: (a) Offset of $+10 \mathrm{mV}$ input into ADC comparator with offset correction turned off, when input is a square wave between $0 \mathrm{~V}$ and $V_{D D} . V_{D D}=V_{R E F}=0.6 \mathrm{~V}$, sample rate $=60 \mathrm{kS} / \mathrm{s}$. (b) Offset of $+10 \mathrm{mV}$ input into $A D C$ comparator with offset correction turned off, when input is a square wave between $0 \mathrm{~V}$ and $V_{D D} . V_{D D}=V_{R E F}=0.6 \mathrm{~V}$, sample rate $=60 \mathrm{kS} / \mathrm{s}$.

digital bits from the ADC can be seen in the oscilloscope image. The analyzed analog input and digitized output is shown in Figure 6.16. The average power dissipation was $180.2 \mathrm{nW}$ over the entire measurement, with an energy per conversion cycle of $18 \mathrm{pJ} /$ cycle.

\subsection{Measured Results of Analog to Digital Con- verter ICGCUKAH}

A photograph of the second ADC chip entitled, ICGCUKAH, is shown in Figure 6.17a. The test setup is shown in Figure 6.17b. The chip was bonded to a PCB using aluminum bond wires and a wedge bonder. The PCB contained decoupling capacitors for the DC pins such as $V_{D D}$ (analog, digital and buffer), $V_{R E F}, I_{b i a s}$, and $V_{t u n e}$. The Analog IN and digital clk pins were connected through standard SMA connectors on the PCB. The pins to reset the digital logic, to select offset correction and to set the 3-bit input for the tunable capacitor was done through a DIP switch on the PCB. 


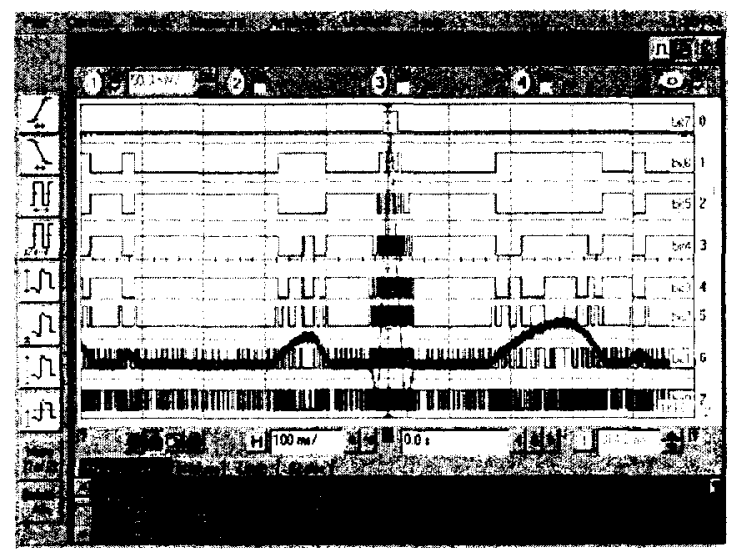

Figure 6.15: Measured output when an analog ECG signal is input into ICGCUADC when supply voltage is $0.4 \mathrm{~V}$ at a sample rate of $10 \mathrm{kS} / \mathrm{s}$ and a $V_{R E F}$ of $0.4 \mathrm{~V}$.
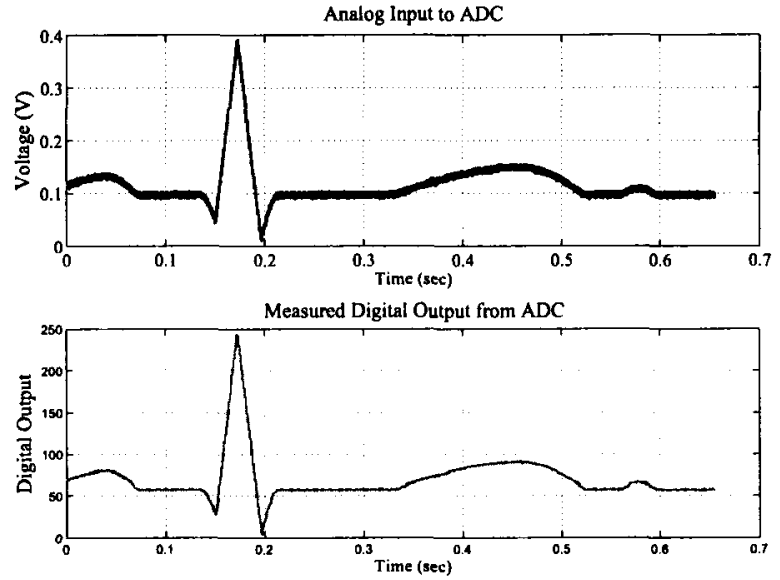

Figure 6.16: Analysis of measured output when an analog ECG signal is input into ICGCUADC when supply voltage is $0.4 \mathrm{~V}$ at a sample rate of $10 \mathrm{kS} / \mathrm{s}$ and a $V_{R E F}$ of $0.4 \mathrm{~V}$. 


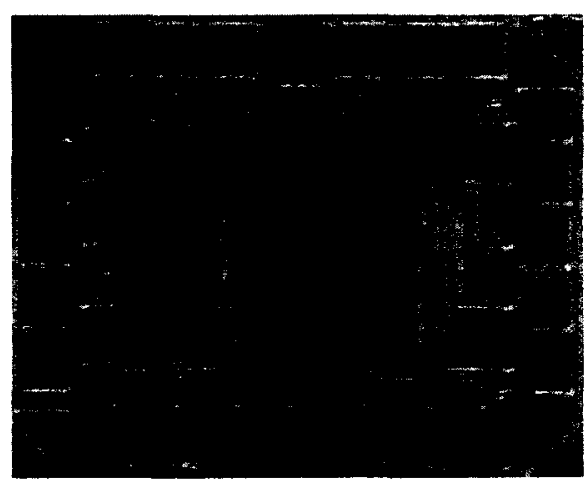

(a)

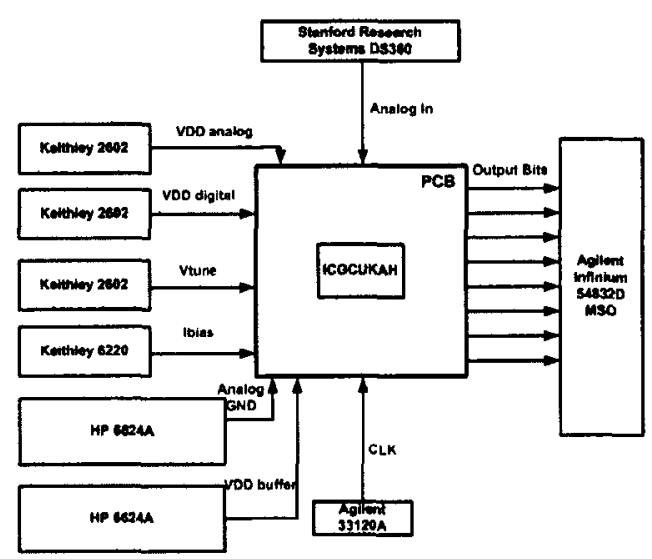

(b)

Figure 6.17: (a) Photograph of ADC ICGCUKAH. (b) Test setup of ICGCUKAH.

There was difficulty in using the low noise DC Keithly source for the analog GND pin as it created a low frequency sine wave as the Keithley source measured power dissipation. Instead a noisier but more stable HP 6624A was used for the analog ground for ICGCUKAH. The PCB also contained ESD protection and zener diodes for the supply voltages on chip, with leakage currents being below 20nA for these components.

\subsubsection{Linearity Results: INL and DNL}

Static measurements for the second ADC chip were conducted by inputting a slow ramp signal into the ADC. There were no missing codes for the ADC. The INL was found to 0.79 LSB and the DNL was found to be 0.93 LSB as shown in Figure 6.18a and $6.18 \mathrm{~b}$ respectively. The tunable attenuation capacitor in the capacitor array was set to the minimum capacitance with a digital input of 000 . This is likely not the exact value required for the capacitor array, as the optimum capacitance is actually less than the minimum obtainable capacitance set by the tunable capacitor. 
A slow sine wave of $20 \mathrm{~Hz}$ was input into the $\mathrm{ADC}$ and the output was examined around when the ADC switches between the 01111111 and 10000000 . As shown in Figure 6.19 the cases for the tunable capacitor digital control states of $000,001,010$ and 011 are shown. For cases 000 and 001 there are no missing codes in the zoomed in area. For the cases 010 and 011 the number of missing codes increases as the attenuation capacitor is increasing, where case 010 is missing code 01101111 and case 011 is missing codes $010111111,01101111,011111111,10001111$ and 10011111 . Cases 000, 001 and 010 have the case when the ADC properly switches between 01111111 and 1000000 while case 011 switches from 01111110 to 10000000 . Larger values for the attenuation capacitance are shown in Figure 6.20, where the cases for the tunable capacitor digital control states of 100,101, 110 and 111 are shown. Again the number of missing codes is significantly increasing as the attenuation capacitor is increasing in size. When the sinusoid is at half its maximum amplitude, case 100 switches from 01111110 to 1000000 skipping 1 code, case 101 switches from 01111101 to 10000000 skipping 2 codes, case 110 switches from 01111101 to 10000000 skipping 2 codes and case 111 switches from 01111100 to 10000000 skipping 3 codes. The worst case in terms of static performance occurs when the attenuation capacitor is the largest, with an input of 111.

The on-chip tunable capacitor for static ADC testing is functional, however the optimum value of the attenuation capacitance is outside the range of what is obtainable by the tunable capacitor. This results in the best available capacitance occurring when it is the smallest, where the digital input is 000 . 


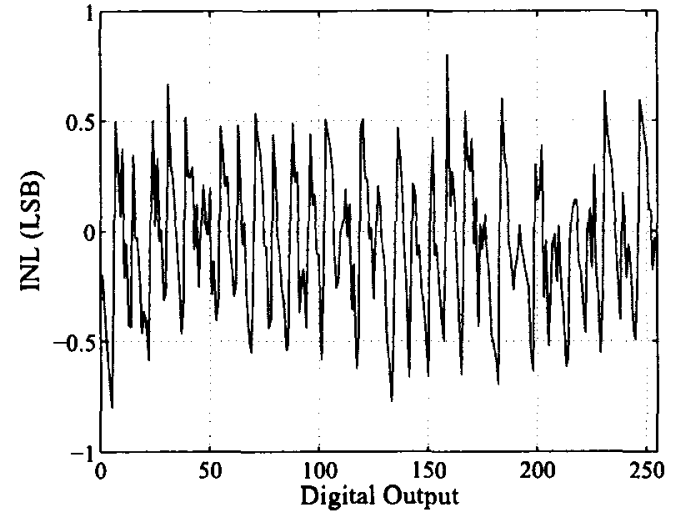

(a)

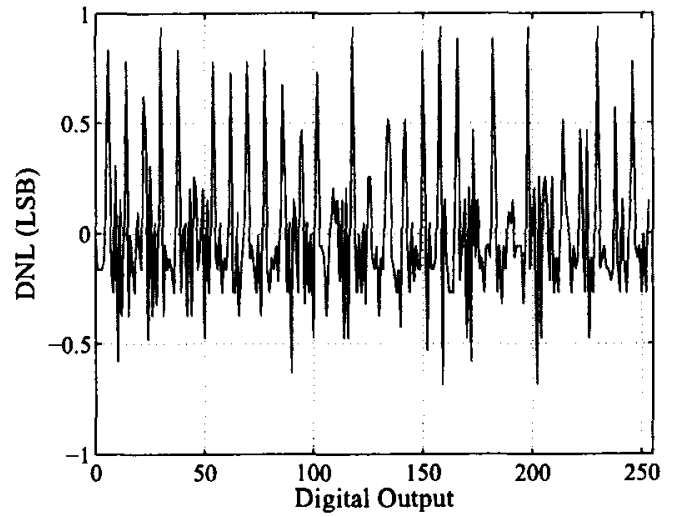

(b)

Figure 6.18: (a) Measured INL plot for ADC ICGCUKAH with a sample rate of $500 \mathrm{kS} / \mathrm{s}$, Vref $=0.5$ and $V_{D D}=1.0 \mathrm{~V}$. (b) Measured DNL plot for ADC ICGCUKAH with a sample rate of $500 \mathrm{kS} / \mathrm{s}, \mathrm{Vref}=0.5$ and $V_{D D}=1.0 \mathrm{~V}$.

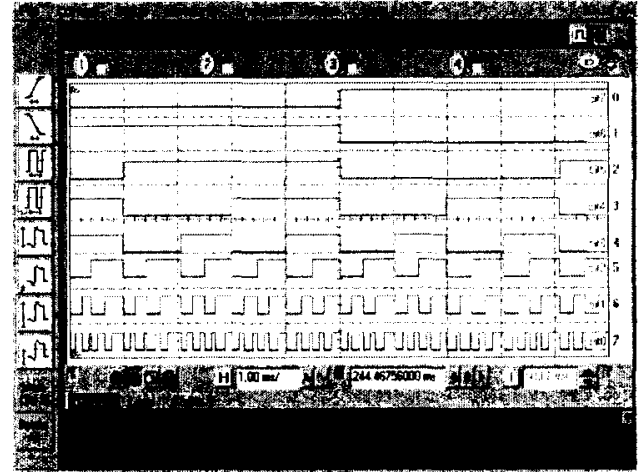

(a)

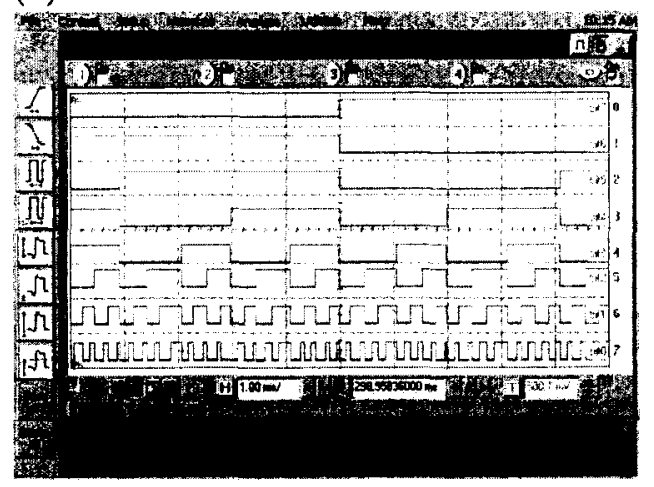

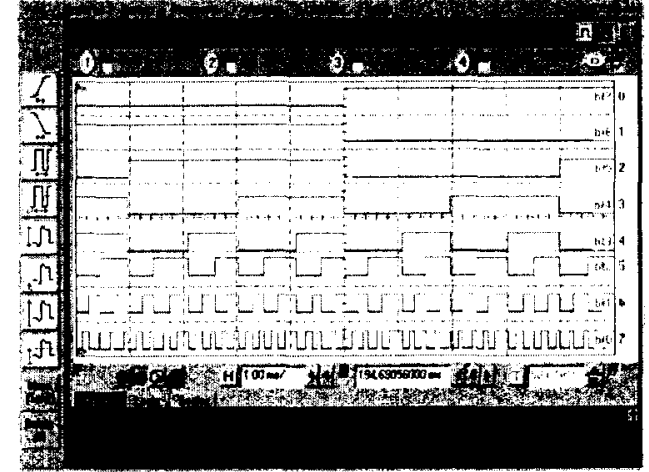

(b)

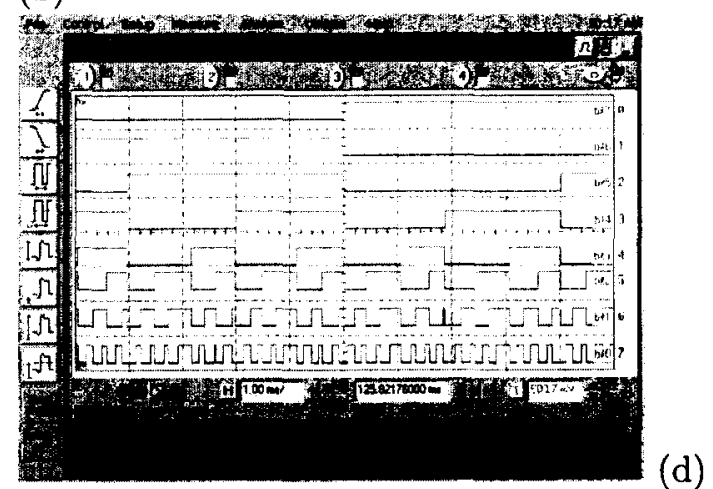

Figure 6.19: Measured digital output zoomed in around 1/2 $V_{R E F}$ for a slow $20 \mathrm{~Hz}$ sine input when capacitor input is equal to (a) 000 (b) 001 (c) 010 (d) 011 for $V_{D D}=0.6 \mathrm{~V}$, sample rate of $20 \mathrm{kS} / \mathrm{s}$. 


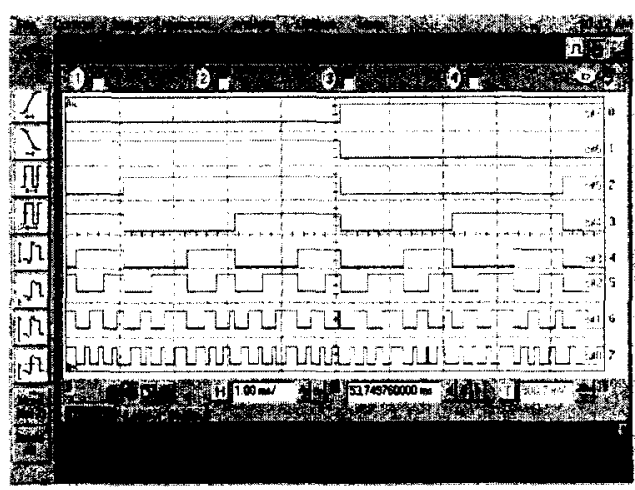

(a)

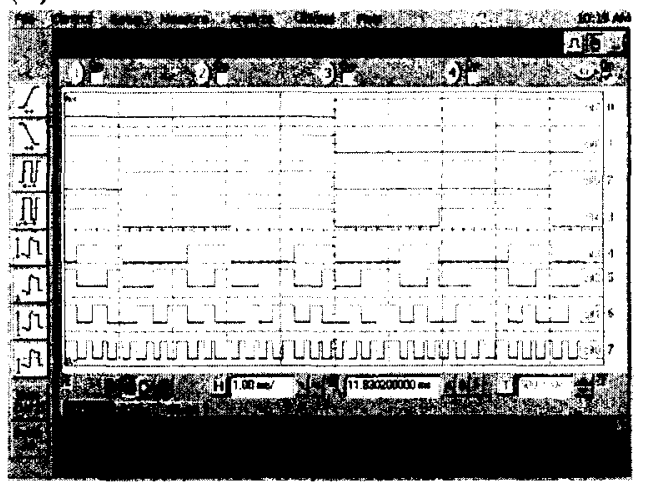

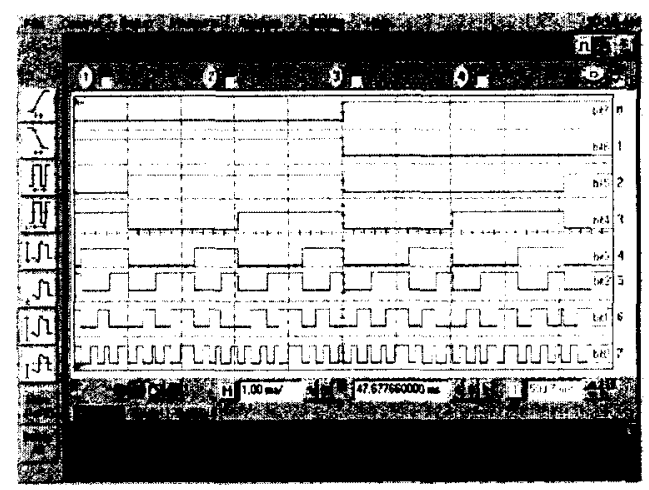

(b)

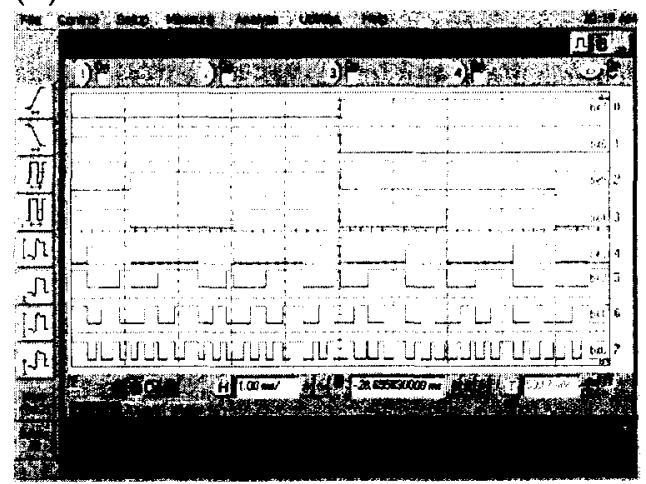

Figure 6.20: Measured digital output zoomed in around 1/2 $V_{R E F}$ for a slow $20 \mathrm{~Hz}$ sine input when capacitor input is equal to (a) 100 (b) 101 (c) 110 (d) 111 for $V_{D D}=0.6 \mathrm{~V}$, sample rate of $20 \mathrm{kS} / \mathrm{s}$. 


\subsubsection{Dynamic Results}

A sinusoid input of $0.3 \mathrm{~V}$ pk-pk at $1000 \mathrm{~Hz}$ was input in the ADC, while sampling at $100 \mathrm{kS} / \mathrm{s}$. The DFT was taken with the output between DC and the Nyquist frequency shown in Figure 6.21, at which the SINAD, ENOB and SFDR can be extracted. This was repeated and plotted for the case when the sample rate is $40 \mathrm{kS} / \mathrm{s}$ for different input frequencies and for each value of the tunable capacitor. Figure 6.22a, (b), (c) and (d) and Figure 6.23a, (b), (c) and (d) show the measured SINAD and SFDR for different input frequencies when $C_{1} C_{2} C_{3}$ is set to $000,001,010,011,100,101$, 110 and 111 respectively. When $C_{1} C_{2} C_{3}$ is set to the smallest capacitance (000), 47.1dB of SINAD is obtainable, with a SFDR of $62.2 \mathrm{~dB}$ which is fairly close to an ideal 8-bit ADC. When the tunable capacitor is the largest (111), 42.3dB of SINAD is obtainable, with a SFDR of $45.6 \mathrm{~dB}$ which is closer to an ideal 7-bit ADC. The other values of the capacitance give results between these two extremes.

Figure $6.24 \mathrm{a}, \mathrm{b}, \mathrm{c}$ and $\mathrm{d}$ and Figure $6.25 \mathrm{a}, \mathrm{b}, \mathrm{c}$ and $\mathrm{d}$ show the measured ENOB for different input frequencies when $C_{1} C_{2} C_{3}$ is set to $000,001,010,011,100,101,110$ and 111 respectively. When $C_{1} C_{2} C_{3}$ is set to the smallest capacitance (000), 7.54 bits is obtainable and when $C_{1} C_{2} C_{3}$ is the largest (111) 6.7 bits is obtainable. The other values of the capacitance give results between these two extremes.

In Figure 6.26 the average ENOB, SINAD and SFDR are taken over all input frequencies between 400 and $9000 \mathrm{~Hz}$ and plotted for each capacitor value. It is shown that a smaller attenuation capacitor will enable a more accurate ADC, and its accuracy decreases as the attenuation capacitor increases in size. Thus, when 


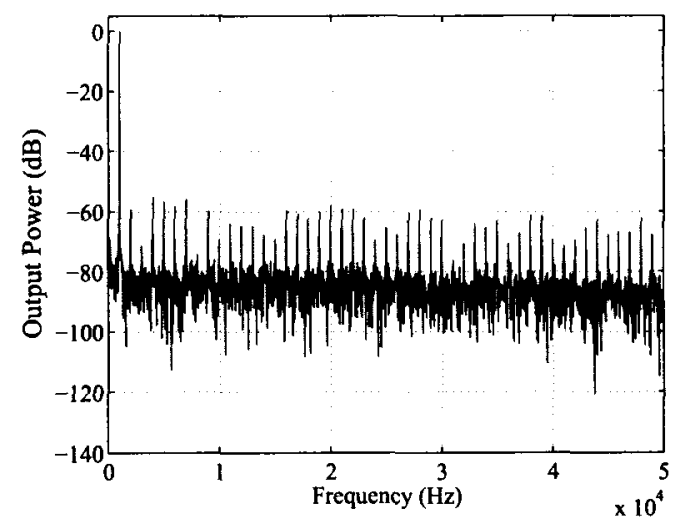

Figure 6.21: DFT of measured output of ADC ICGCUKAH, when input is a $1 \mathrm{kHz}$, $0.3 \mathrm{~V}$ pk-pk Sinusoid. For the ADC, $V_{D D}=V_{R E F}=0.6 \mathrm{~V}$ and sample rate $=100 \mathrm{kS} / \mathrm{s}$.

$C_{1} C_{2} C_{3}=$ '000' the ADC offers the best performance with an average ENOB of 7.1 bits for an input frequency between 400 and $9000 \mathrm{~Hz}$ and when $C_{1} C_{2} C_{3}=$ '111' this enables the least accurate ADC for all capacitance values of the ADC, with an average ENOB of 6.27 bits for an input frequency between 400 and $9000 \mathrm{~Hz}$. The optimum capacitance is likely smaller than the capacitance achieved when $C_{1} C_{2} C_{3}={ }^{\prime} 000$ '.

\subsubsection{Power Dissipation}

The power dissipation for the analog circuitry increases much less than the case for the first $\mathrm{ADC}$ chip with an increasing sample rate as the two switches required to reset the top plate of the capacitor array use transmission gates, which removes the extra capacitance that needs to charged and discharged every cycle. The slight increase in analog power dissipation with sample rate is mainly due to the faster charging and discharging of the capacitor array, as well as the offset correction circuitry. The digital circuitry and thus its power dissipation is identical to first ADC, ICGCUADC.

Shown in Figure 6.27a, (b) and (c) is the power dissipation versus sample rate for 


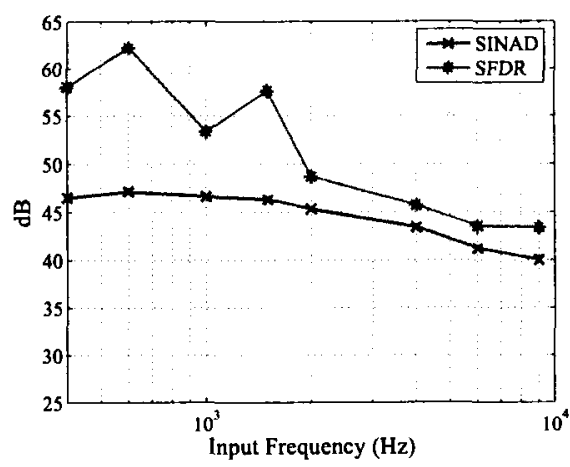

(a)

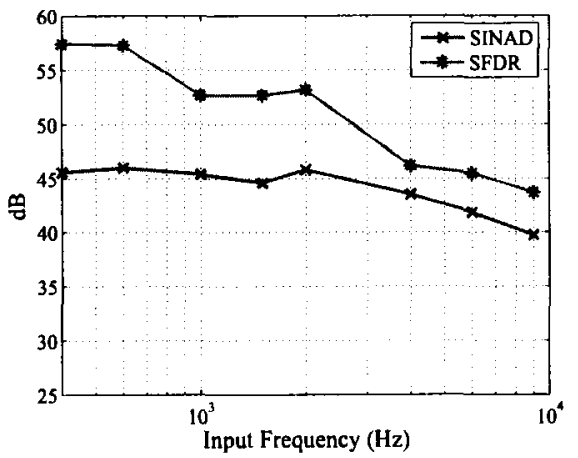

(c)

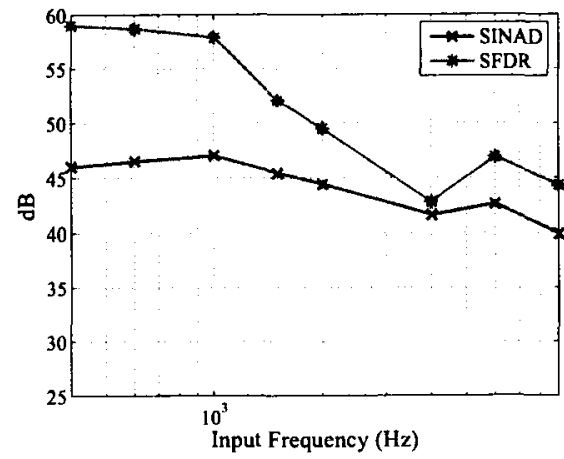

(b)

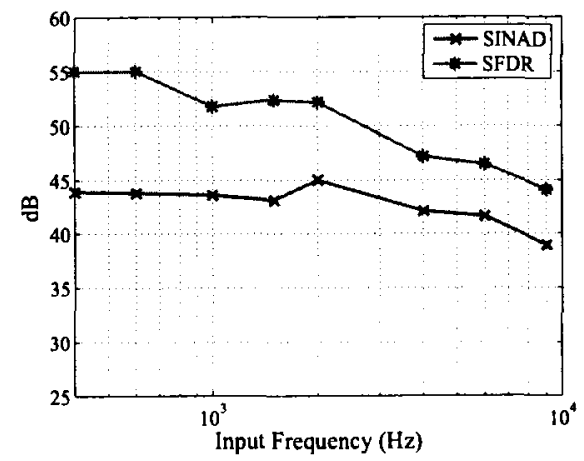

(d)

Figure 6.22: Measured SINAD and SFDR for different input frequencies when capacitor input is equal to (a) 000 (b) 001 (c) 010 (d) 011 , with $V_{D D}=0.6 \mathrm{~V}$, sample rate of $40 \mathrm{kS} / \mathrm{s}$. 


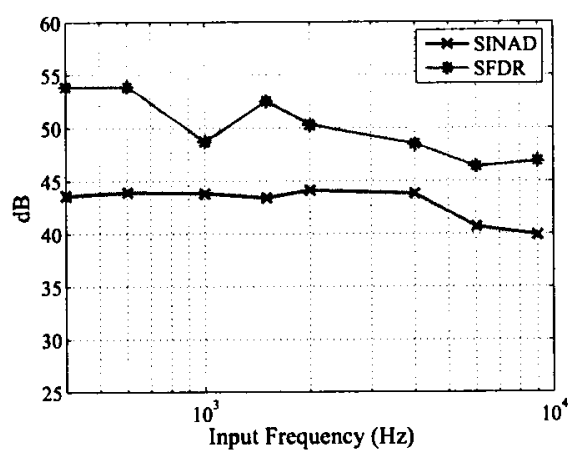

(a)

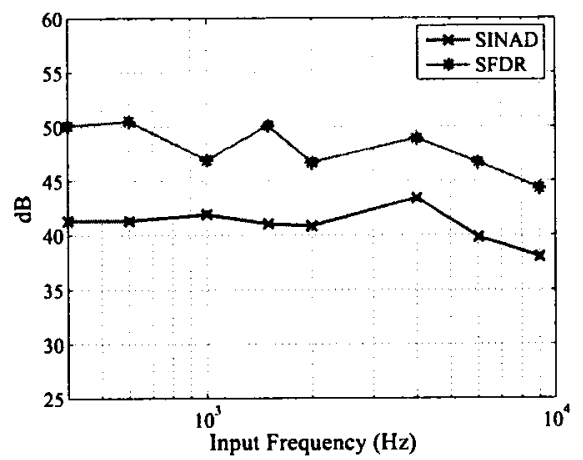

(c)

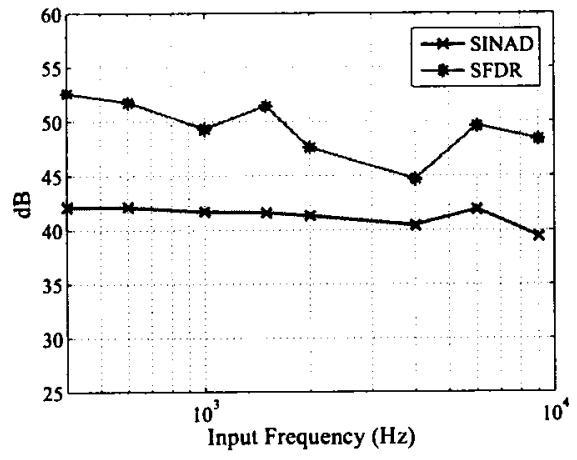

(b)

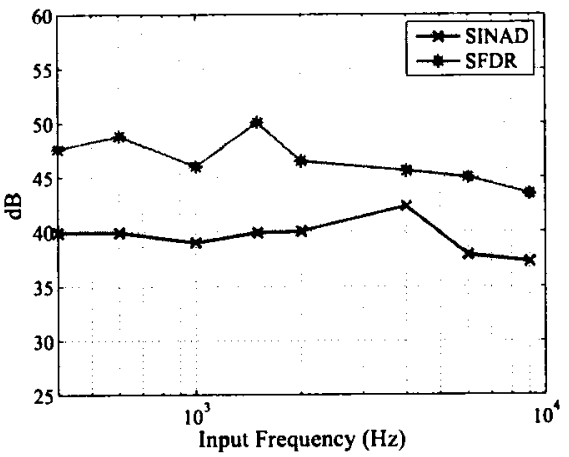

(d)

Figure 6.23: Measured SINAD and SFDR for different input frequencies when capacitor input is equal to (a) 100 (b) 101 (c) 110 (d) 111 , with $V_{D D}=0.6 \mathrm{~V}$, sample rate of $40 \mathrm{kS} / \mathrm{s}$. 


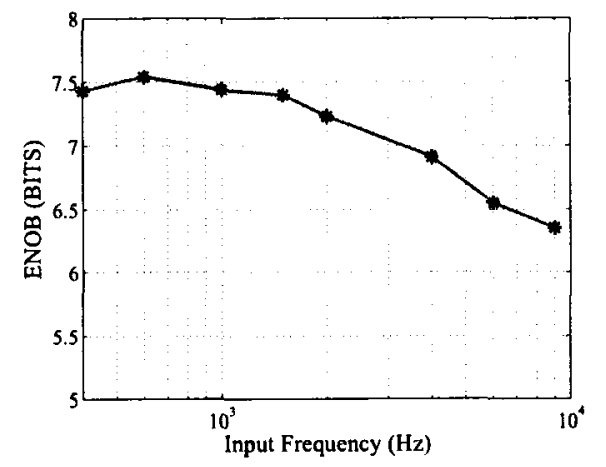

(a)

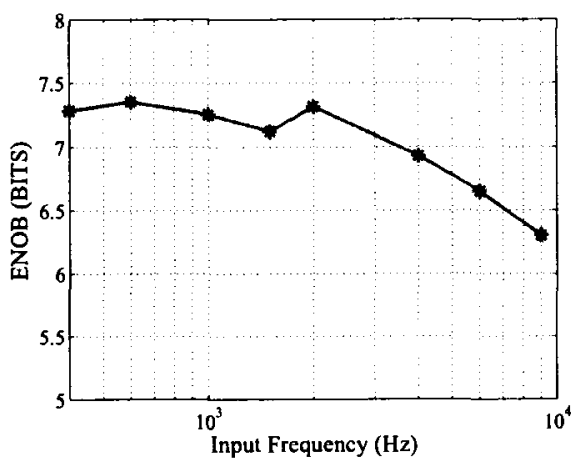

(c)

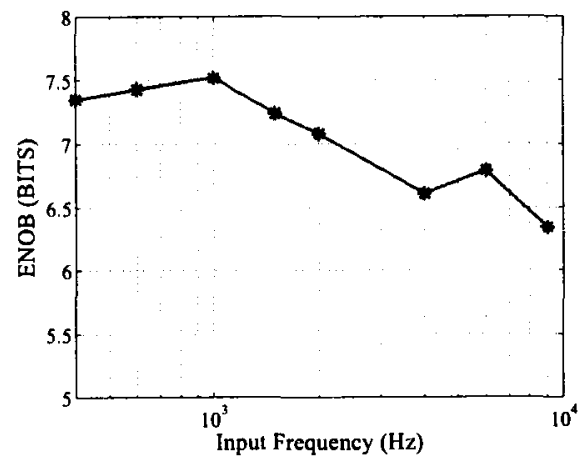

(b)

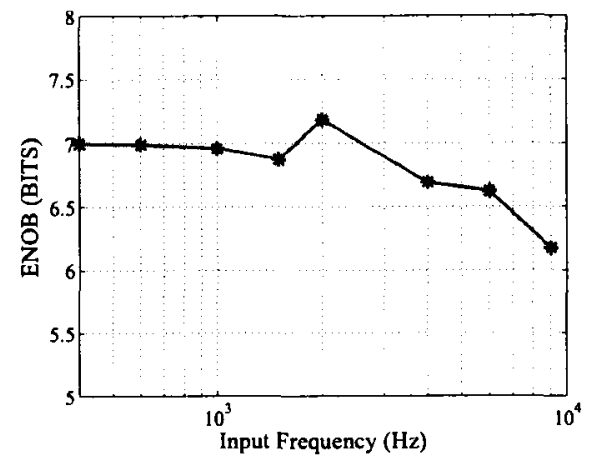

(d)

Figure 6.24: Measured ENOB for different input frequencies when capacitor input is equal to (a) 000 (b) 001 (c) 010 (d) 011 , with $V_{D D}=0.6 \mathrm{~V}$, sample rate of $40 \mathrm{kS} / \mathrm{s}$. 


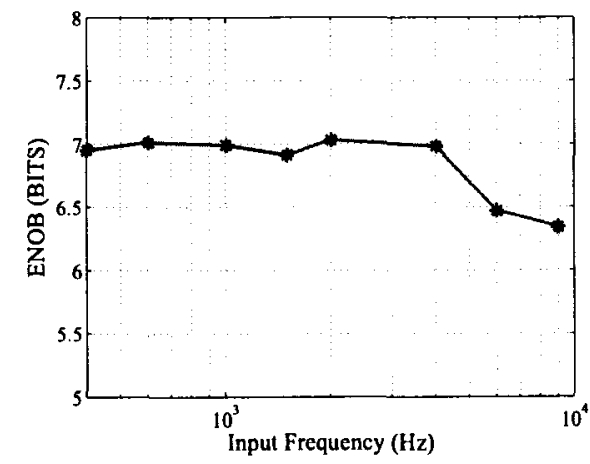

(a)

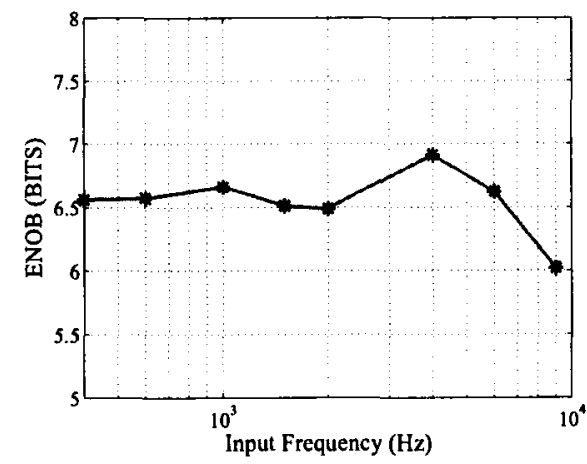

(c)

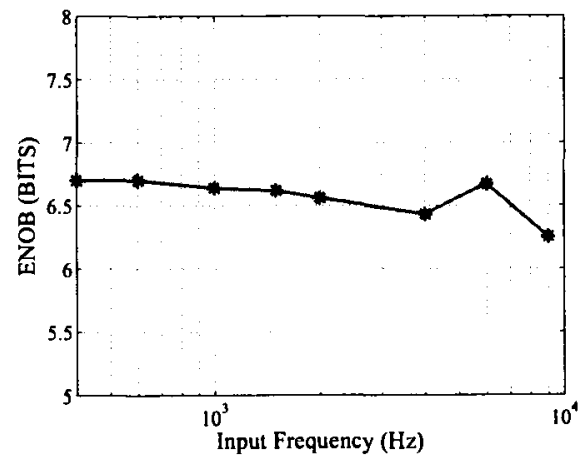

(b)

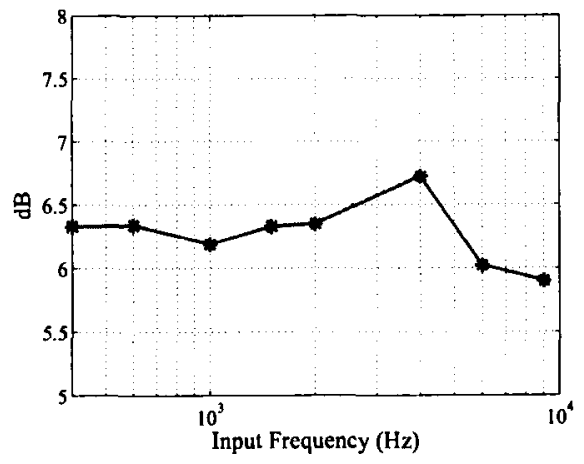

Figure 6.25: Measured ENOB for different input frequencies when capacitor input is equal to (a) 100 (b) 101 (c) 110 (d) 111 , with $V_{D D}=0.6 \mathrm{~V}$, sample rate of $40 \mathrm{kS} / \mathrm{s}$.

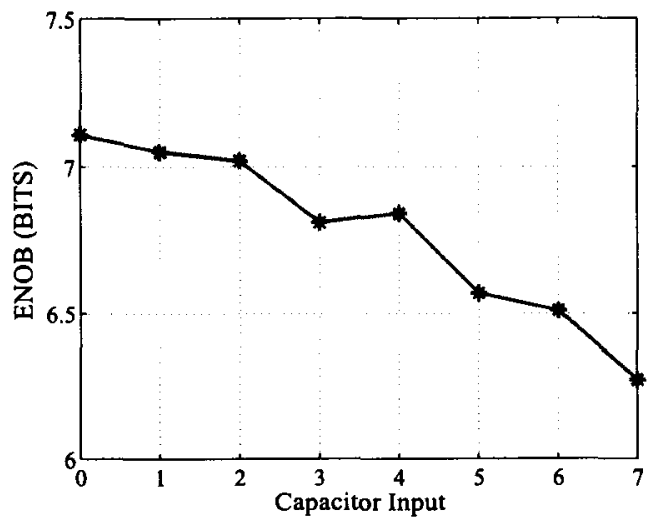

(a)

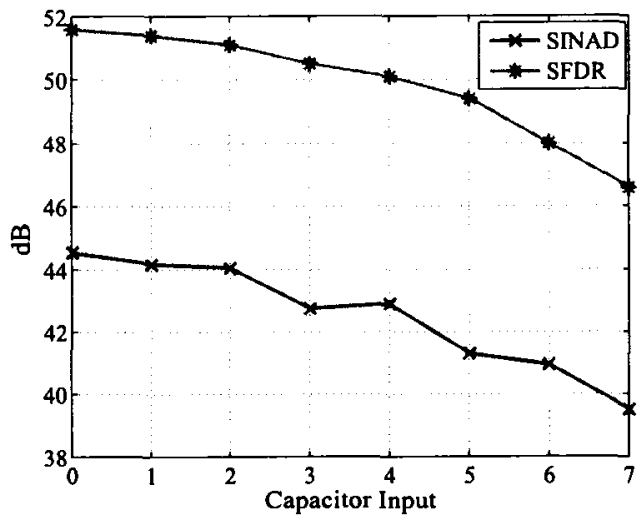

(b)

Figure 6.26: (a) Average measured ENOB of different input frequencies for each capacitor input. (b) Average measured SINAD and SFDR for different input frequencies for each capacitor input. $V_{D D}=0.6 \mathrm{~V}$, sample rate of $40 \mathrm{kS} / \mathrm{s}$. 
supply voltages of $0.4,0.6$ and $0.8 \mathrm{~V}$ respectively. The measured power dissipation for all three cases is similar to the simulated power dissipation. For a $V_{D D}$ of $0.4 \mathrm{~V}$ the simulated power dissipation at $20 \mathrm{kS} / \mathrm{s}$ is $170 \mathrm{nW}$, while the measured power dissipation is $161.8 \mathrm{nW}$. For a $V_{D D}$ of $0.6 \mathrm{~V}$ the simulated power dissipation at $100 \mathrm{kS} / \mathrm{s}$ is $950 \mathrm{nW}$, while the measured power dissipation is $948 \mathrm{nW}$. For a $V_{D D}$ of $0.8 \mathrm{~V}$ the simulated power dissipation at $400 \mathrm{kS} / \mathrm{s}$ is $4900 \mathrm{nW}$, while the measured power dissipation is $4144 \mathrm{nW}$.

The energy per conversion cycle is shown in Figure 6.28. The best case energy per conversion cycle is for sub-threshold operation at a $0.4 \mathrm{~V}$ supply where the ADC uses $8.09 \mathrm{pJ}$. For a 0.6 and $0.8 \mathrm{~V}$ supply the ADC uses $13.45 \mathrm{pJ}$ and $15.05 \mathrm{pJ}$ per conversion cycle respectively. This corresponds to the simulation where for supplies of $0.4 \mathrm{~V}$, $0.6 \mathrm{~V}$ and $0.8 \mathrm{~V}$ the energy per conversion cycle as seen in simulation was $8.5 \mathrm{pJ}, 9.5 \mathrm{pJ}$ and $12.3 \mathrm{pJ}$ respectively. A 50 iteration Monte Carlo simulation for a $60 \mathrm{kS} / \mathrm{s}$ sample rate had an energy per conversion cycle varied between $13.2 \mathrm{pJ}$ and $17.4 \mathrm{pJ}$ when a $0.6 \mathrm{~V}$ supply is used, with the measured result $(13.45 \mathrm{pJ})$ falling within this range.

\subsubsection{Application Example}

An ECG was input from the Agilent source into the ADC sampled at $10 \mathrm{kS} / \mathrm{s}$ with a $V_{D D}$ of $0.4 \mathrm{~V}$ and a $V_{R E F}$ of $0.2 \mathrm{~V}$. The analyzed analog input and digitized output are shown in Figure 6.29. The average power dissipation was $150 \mathrm{nW}$ over the entire measurement, with an energy per conversion cycle of $15 \mathrm{pJ} /$ cycle. 


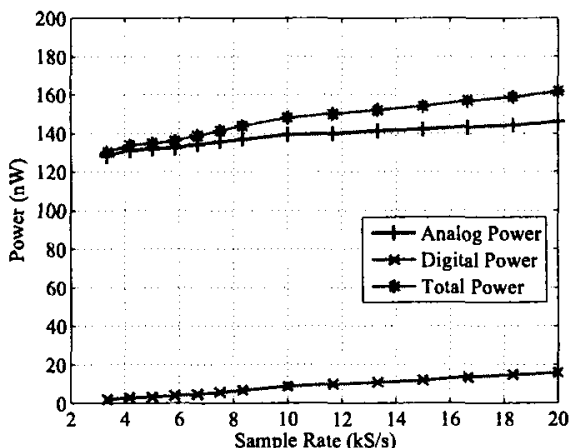

(a)

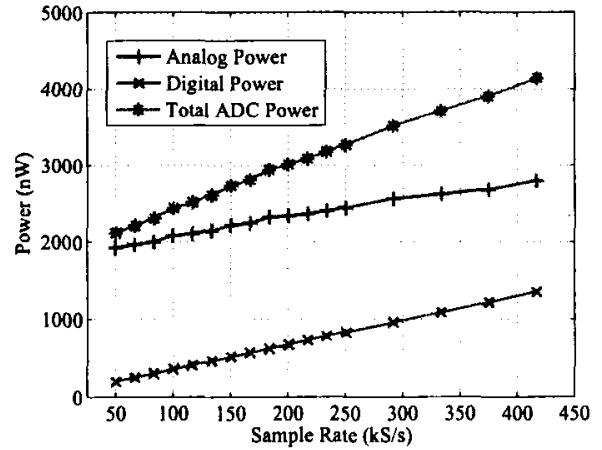

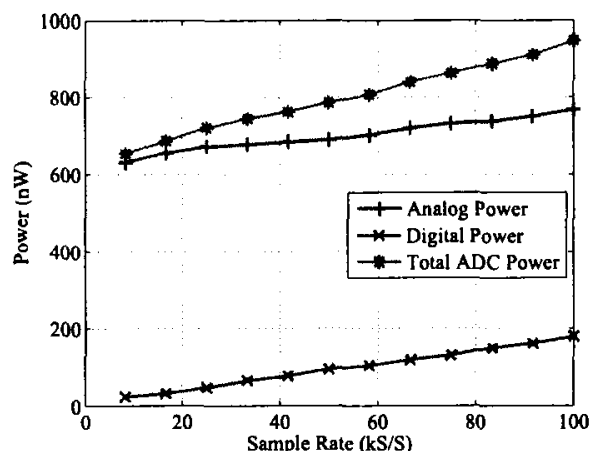

(b)

(c)

Figure 6.27: (a) Measured Power dissipation of full ADC ICGCUKAH vs. sample rate for $V_{D D}=0.4 \mathrm{~V}$ (b) Measured Power dissipation of full ADC ICGCUKAH vs. sample rate for $V_{D D}=0.6 \mathrm{~V}$ (c) Measured Power dissipation of full ADC ICGCUKAH vs. sample rate for $V_{D D}=0.8 \mathrm{~V}$ 


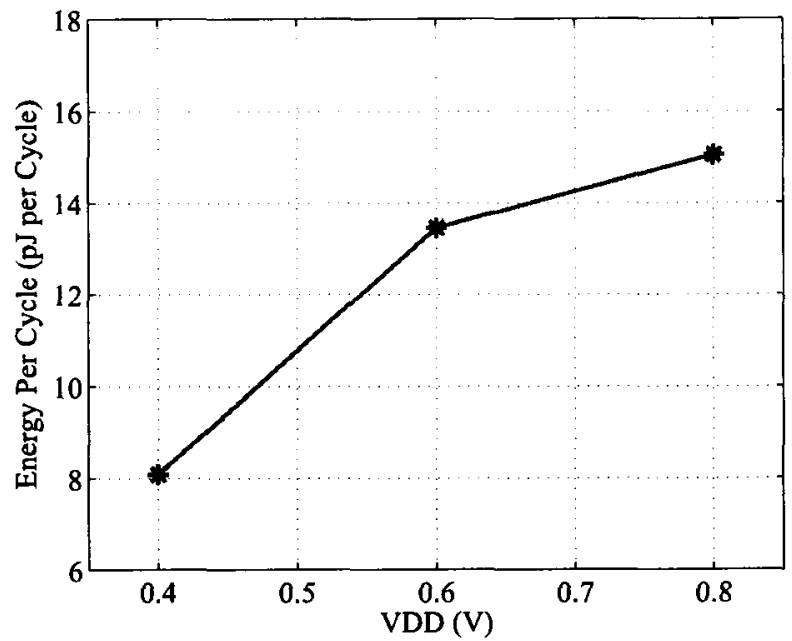

Figure 6.28: Measured energy per cycle of full ADC ICGCUKAH vs. $V_{D D}$. Sample rate $=20 \mathrm{kS} / \mathrm{s}, 60 \mathrm{kS} / \mathrm{s}$, and $200 \mathrm{kS} / \mathrm{s}$ for $V_{D D}$ of $0.4,0.6$ and $0.8 \mathrm{~V}$ respectively.
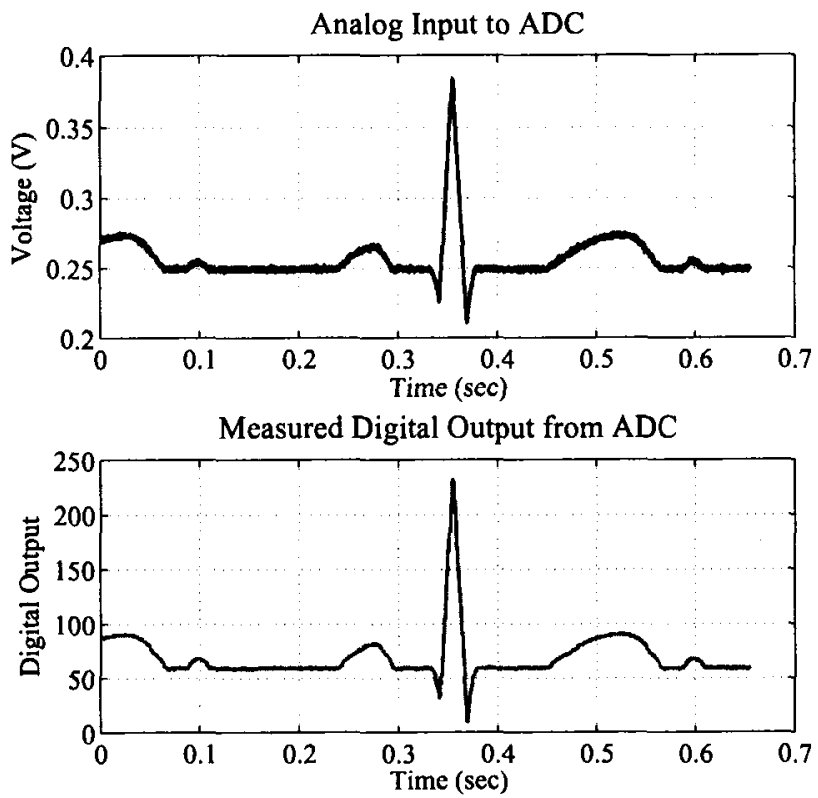

Figure 6.29: Analysis of measured output when an analog ECG signal is input into ADC ICGCUKAH when supply voltage is $0.4 \mathrm{~V}$ at a sample rate of $10 \mathrm{kS} / \mathrm{s}$ and a $V_{R E F}$ of $0.2 \mathrm{~V}$. 


\subsection{Summary of Measurement Results}

The measured results for the ADC, ICGCUADC are summarized in Table 6.1 and for the second ADC, ICGCUKAH in Table 6.2.

\subsubsection{Energy Efficiency}

ICGCUKAH achieves a lower measured energy per conversion cycle than ICGCUADC, with $8.09 \mathrm{pJ}$ per cycle versus $10.3 \mathrm{pJ}$ per cycle, $13.45 \mathrm{pJ}$ per cycle versus $16.8 \mathrm{pJ}$ per cycle and $15.05 \mathrm{pJ}$ per cycle versus $23.6 \mathrm{pJ} /$ cycle for supply voltages of $0.4,0.6$ and $0.8 \mathrm{~V}$ respectively. It should be noted though, that ICGCUKAH does not have a full input range, and thus requires an additional analog GND, which would likely be a bandgap voltage reference, further increasing the power dissipation.

\subsubsection{Accuracy}

The attenuation capacitor as simulated turned out to be too large when fabricated resulting in ICGCUADC having missing codes, and a maximum ENOB of slightly over 7 bits. Similarly for ICGCUKAH, the attenuation capacitor turned out to be too large, which resulted in the tunable capacitor having its best-case capacitance at its minimum value. This allowed the ADC to improve over ICGCUADC by having no missing codes, and INL and DNL below one LSB and a maximum ENOB of 7.54 bits.

\subsubsection{Sensitivity}

ICGCUKAH which has one half of the input range as ICGCUADC, is much more sensitive to external noise from equipment and the PCB. A low noise Keithly supply 
was used to provide an analog GND. However there was a $1 \mathrm{mV} 100 \mathrm{~Hz}$ ripple being output by this source which was coupled into the ADC output. Consequently, a replacement source without the ripple but unfortunately with higher noise was used. For ICGCUKAH, when lower values of $V_{D D}$ are used such as $0.4 \mathrm{~V}$, the LSB becomes only $780 \mathrm{uV}$ making it difficult to obtain accurate results allowing for a maximum ENOB of 6.6 bits.

\subsection{Comparison to Published Results}

A survey of recent IEEE journal and conference papers on ultra-low power ADCs is shown in Table 6.3. Both ADCs, ICGCUADC and ICGCUKAH are one of the most energy efficient ADCs in terms of energy per conversion cycle, used as an energy efficiency benchmark in [2], energy per bit, used as a energy efficiency benchmark in [21], and in terms of energy per state, used as a energy efficiency benchmark in [23]. Currently, the most ultra-low power energy efficient ADC designs are [14], [2], [11] and [21]. In terms of energy per conversion cycle the ADC ICGCUKAH, when operating with a $0.4 \mathrm{~V}$ supply offers the most energy efficient at $8.09 \mathrm{pJ} /$ cycle. In terms of energy per bit, both ADCs ICGCUKAH and ICGCUADC offer the most energy efficient at $1.22 \mathrm{pJ}$ and $1.54 \mathrm{pJ}$ per bit, respectively. In terms of energy per state (or quantization level), ICGCUKAH offers the most energy efficient ADC at $0.0725 \mathrm{pJ}$ per state and $0.0811 \mathrm{pJ}$ per state for a supply of $0.6 \mathrm{v}$ and $0.4 \mathrm{~V}$, respectively.

The ADC in [14] offers comparable performance in terms of energy efficiency however, it uses a more exotic Silicon-on-Saphire process instead of a standard CMOS process which makes integration less cost effective. The most energy efficient 8-bit 


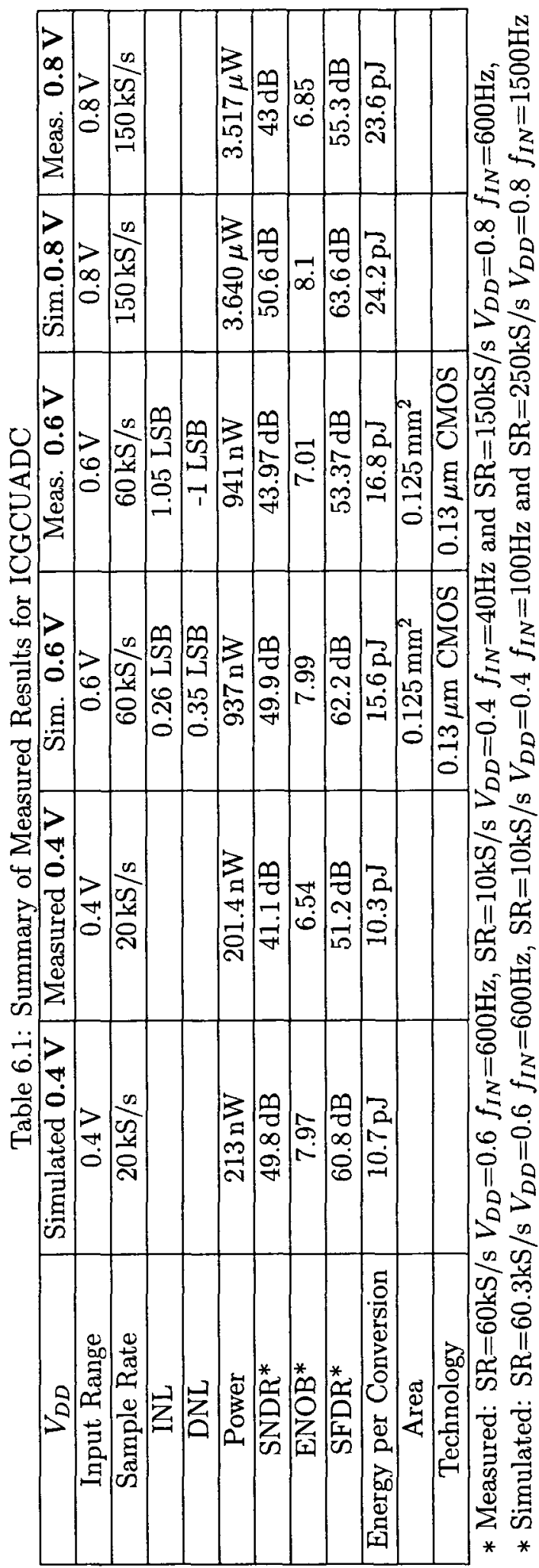


Table 6.2: Summary of Measured Results for ICGCUKAH

\begin{tabular}{|c|c|c|c|c|c|c|}
\hline$V_{D D}$ & Simulated 0.4 V & Measured 0.4 V & Sim. 0.6 V & Meas. 0.6 V & Sim. 0.8 V & Meas. 0.8 V \\
\hline Input Range & $0.2 \mathrm{~V}$ & $0.2 \mathrm{~V}$ & $0.3 \mathrm{~V}$ & $0.3 \mathrm{~V}$ & $0.4 \mathrm{~V}$ & $0.4 \mathrm{~V}$ \\
\hline Sample Rate & $20 \mathrm{kS} / \mathrm{s}$ & $20 \mathrm{kS} / \mathrm{s}$ & $100 \mathrm{kS} / \mathrm{s}$ & $60 \mathrm{kS} / \mathrm{s}$ & $400 \mathrm{kS} / \mathrm{s}$ & $200 \mathrm{kS} / \mathrm{s}$ \\
\hline INL & & & $0.38 \mathrm{LSB}$ & $0.79 \mathrm{LSB}$ & & \\
\hline DNL & & & $0.83 \mathrm{LSB}$ & $0.93 \mathrm{LSB}$ & & \\
\hline Power & $170 \mathrm{nW}$ & $161.8 \mathrm{nW}$ & $950 \mathrm{nW}$ & $807 \mathrm{nW}$ & $4.94 \mu \mathrm{W}$ & $3.010 \mu \mathrm{W}$ \\
\hline SNDR $^{*}$ & & $41.6 \mathrm{~dB}$ & $49.9 \mathrm{~dB}$ & $47.1 \mathrm{~dB}$ & & $46 \mathrm{~dB}$ \\
\hline ENOB $^{*}$ & & 6.62 & 7.99 & 7.54 & & 7.35 \\
\hline SFDR & & $47.8 \mathrm{~dB}$ & $56.2 \mathrm{~dB}$ & $62.2 \mathrm{~dB}$ & & $60.7 \mathrm{~dB}$ \\
\hline Energy per Conversion & $8.5 \mathrm{pJ}$ & $8.09 \mathrm{pJ}$ & $9.5 \mathrm{pJ}$ & $13.45 \mathrm{pJ}$ & $12.3 \mathrm{pJ}$ & $15.05 \mathrm{pJ}$ \\
\hline Area & & & $0.125 \mathrm{~mm}^{2}$ & $0.125 \mathrm{~mm}^{2}$ & & \\
\hline Technology & & & $0.13 \mu \mathrm{m} \mathrm{CMOS}_{\mathrm{CMO}}$ & $0.13 \mu \mathrm{m} \mathrm{CMOS}$ & & \\
\hline
\end{tabular}

* Measured: $\mathrm{SR}=60 \mathrm{kS} / \mathrm{s} V_{D D}=0.6 f_{I N}=600 \mathrm{~Hz}, \mathrm{SR}=10 \mathrm{kS} / \mathrm{s} V_{D D}=0.4 f_{I N}=40 \mathrm{~Hz}$ and $\mathrm{SR}=200 \mathrm{kS} / \mathrm{s} V_{D D}=0.8 f_{I N}=2000 \mathrm{~Hz}$

* Simulated: $\mathrm{SR}=60.3 \mathrm{kS} / \mathrm{s} V_{D D}=0.6 f_{I N}=1000 \mathrm{~Hz}$ 
ADC in a standard CMOS process was [11], which offers $20 \mathrm{pJ}$ per cycle, $2.7 \mathrm{pJ}$ per bit and $0.118 \mathrm{pJ}$ per state. ICGCUADC offers a more energy efficient design than [11] with $10.1 \mathrm{pJ}$ per cycle, $1.54 \mathrm{pJ}$ per bit and $0.109 \mathrm{pJ}$ per state when a $0.4 \mathrm{~V}$ supply is used and ICGCUKAH offers an even more energy efficient ADC design with 8.09pJ per cycle, $1.22 \mathrm{pJ}$ per bit and $0.0811 \mathrm{pJ}$ per state when a $0.4 \mathrm{~V}$ supply is used. 
Table 6.3: Comparison to published results.

\begin{tabular}{|c|c|c|c|c|c|c|c|}
\hline Arch. & ENOB & Speed & Power & Tech. & Energy* & Energy** & Energy*** \\
\hline SAR [2] & 7.9 & $100 \mathrm{kS} / \mathrm{s}$ & $3.1 \mu \mathrm{W}$ & $0.25 \mu \mathrm{m}$ CMOS & 31pJ/cycle & $3.92 \mathrm{pJ} / \mathrm{bit}$ & $0.13 \mathrm{pJ} /$ state \\
\hline SAR [12] & 6.95 & $4.1 \mathrm{kS} / \mathrm{s}$ & $0.85 \mu \mathrm{W}$ & $0.18 \mu \mathrm{m}$ CMOS & 207pJ/cycle & 30.1pJ/bit & $1.67 \mathrm{pJ} / \mathrm{state}$ \\
\hline SAR [13] & 8 & $1 \mathrm{kS} / \mathrm{s}$ & $300 \mathrm{nW}$ & $0.5 \mu \mathrm{m}$ CMOS & $300 \mathrm{pJ} /$ cycle & $37.5 \mathrm{pJ} / \mathrm{bit}$ & $1.17 \mathrm{pJ} /$ state \\
\hline Int [11] & 7.4 & $45 \mathrm{kS} / \mathrm{s}$ & $900 \mathrm{nW}$ & $0.18 \mu \mathrm{m}$ CMOS & $20 \mathrm{pJ} /$ cycle & $2.7 \mathrm{pJ} / \mathrm{bit}$ & $0.118 \mathrm{pJ} /$ state \\
\hline SAR [14] & 7.6 & $409 \mathrm{kS} / \mathrm{s}$ & $7.3 \mu \mathrm{W}$ & $0.5 \mu \mathrm{m}$ SOS & $17.9 \mathrm{pJ} / \mathrm{cycle}$ & $2.35 \mathrm{pJ} / \mathrm{bit}$ & $0.0923 \mathrm{pJ} /$ state \\
\hline SAR [21] & 6 & $1.5 \mathrm{MS} / \mathrm{s}$ & $14 \mu \mathrm{W}$ & $0.09 \mu \mathrm{m}$ CMOS & $9.33 \mathrm{pJ} /$ cycle & $1.56 \mathrm{pJ} / \mathrm{bit}$ & $0.145 \mathrm{pJ} /$ state \\
\hline Int [23] & 8.4 & $2.9 \mathrm{kS} / \mathrm{s}$ & $17 . \overline{9} \mu \mathrm{W}$ & $0.8 \mu \mathrm{m} \mathrm{BiCMOS}$ & $6170 \mathrm{~nJ} /$ cycle & $735 \mathrm{pJ} / \mathrm{bit}$ & $18.3 \mathrm{pJ} /$ state \\
\hline \multicolumn{8}{|l|}{ This Work } \\
\hline ICGCUADC & 7.01 & $60 \mathrm{kS} / \mathrm{s}$ & $94 \operatorname{lnW}$ & $0.13 \mu \mathrm{m}$ CMOS & $15.7 \mathrm{pJ} / \mathrm{cycle}$ & $2.24 \mathrm{pJ} / \mathrm{bit}$ & $0.122 \mathrm{pJ} /$ state \\
\hline ICGCUKAH & 7.54 & $60 \mathrm{kS} / \mathrm{s}$ & $807 \mathrm{nW}$ & $0.13 \mu \mathrm{m}$ CMOS & $13.5 \mathrm{pJ} / \mathrm{cycle}$ & $1.79 \mathrm{pJ} /$ bit & $0.0725 \mathrm{pJ} /$ state \\
\hline$\overline{\text { ICGCUADC }}$ & 6.54 & $20 \mathrm{kS} / \mathrm{s}$ & $201.4 \mathrm{nW}$ & $0.13 \mu \mathrm{m}$ CMOS & 10.1pJ/cycle & $1.54 \mathrm{pJ} / \mathrm{bit}$ & $0.109 \mathrm{pJ} /$ state \\
\hline ICGCUKAH & 6.64 & $20 \mathrm{kS} / \mathrm{s}$ & $161.8 \mathrm{nW}$ & $0.13 \mu \mathrm{m}$ CMOS & $8.09 \mathrm{pJ} / \mathrm{cycle}$ & $1.22 \mathrm{pJ} / \mathrm{bit}$ & $0.0811 \mathrm{pJ} /$ state \\
\hline & & & & & $\frac{\text { Power }}{\text { SampleRate }}$ & & \\
\hline
\end{tabular}




\section{Chapter 7}

\section{Conclusion}

\subsection{Contributions to Research}

The work presented in this thesis demonstrated five main points.

Firstly, that the sub-threshold region of operation of a MOSFET can be used to operate $\mathrm{ADCs}$ at voltages as low as $0.3 \mathrm{~V}$ and demonstrate the lowest energy per conversion cycle for published ADCs. The measured energy per conversion cycle was as low as $8.09 \mathrm{pJ} /$ conversion cycle and $10.45 \mathrm{pJ} /$ conversion cycle for ADCs ICGCUKAH and ICGCUADC respectively.

Secondly, that the tunable capacitor as described in this thesis can be used to tune the attenuation capacitor in a capacitor array to obtain more accurate static and dynamic results for the ADC, ICGCUKAH. This improved the INL, DNL, ENOB, SNR and SFDR when compared to the other ADC, ICGCUADC, which used a fixed attenuation capacitor. The tunable attenuation capacitor allowed ADC, ICGCUKAH to achieve 7.54 bits and $62.2 \mathrm{~dB}$ for ENOB and SFDR respectively compared with ICGCUADC which had 7.0 bits and $53.4 \mathrm{~dB}$ for ENOB and SFDR respecitively. 
Table 7.1: Comparison between ICGCUADC and ICGCUKAH

\begin{tabular}{|c|c|c|}
\hline Specification & ADC 1 & ADC 2 \\
\hline Chip Name & ICGCUADC & ICGCUKAH \\
\hline Analog GND & $0 \mathrm{~V}$ & $V_{D D} / 2$ \\
\hline Input Range & $0-V_{D D}$ & $0.5 V_{D D}-V_{D D}$ \\
\hline Attenuation Capacitor & Fixed & Tunable \\
\hline Comparator Input Transistors & Zero Threshold NFETs & Standard NFETs \\
\hline
\end{tabular}

Thirdly, that the low leakage switch enabled ADC, ICGCUADC to obtain a fullinput swing by pre-charging a reference capacitor to negative $V_{D D}$ before the conversion cycle begins. This extended the input range and enabled the ADC to accept any analog input voltage between GND and $V_{D D}$.

Fourthly, the sub-threshold offset correction used in both ADC designs significantly improved the accuracy of the comparator and made it less sensitive to bias and $V_{D D}$ variations. This was demonstrated by manually inserting an offset into the comparator and examining the effect of this offset on the ADCs output.

Lastly, that both ADCs presented in this thesis converted an ECG signal while dissipating only nanowatts of power, making them good candidates for biomedical applications such as implantable chips.

The measured results for both ADCs were in agreement with simulation results generated during the design process. The design ICGCUKAH offers better performance than ICGCUADC, however, this ADC requires the addition of a stable analog GND voltage which makes it lack the ability to use a full input range. The ADC ICGCUADC exhibits higher power dissipation, but it has the ability to share the chip GND with analog GND, allowing for a full input range while simplifying the bias circuitry. A summary of the differences between the two chips is seen in Table 7.1. 


\subsection{Publications Resulting from this Work}

As a result of the research presented in this thesis, the following papers were published and presented at international conferences on integrated circuits and systems.

1) K. Abdelhalim, L. MacEachern, and S. Mahmoud, A Nanowatt Successive Approximation ADC with a Calibrated Capacitor Array for Biomedical Applications, Proc. of IEEE International Midwest Symposium on Circuits and Systems/IEEE International Northeast Workshop on Circuits and Systems (MWSCAS/NEWCAS), Montreal, Canada 2007. (in press)

2)K. Abdelhalim, L. MacEachern, and S. Mahmoud, A Nanowatt Successive Approximation ADC with Offset Correction for Implantable Sensor Applications, in ISCAS '07. Proceedings of the 2007 International Symposium on Circuits and Systems, New Orleans, USA, 2007, pp. 2351-2354.

3) K. Abdelhalim, L. MacEachern, and S. Mahmoud, A Nanowatt ADC for UltraLow-Power Applications, in ISCAS '06. Proceedings of the 2006 International Symposium on Circuits and Systems, Kos Island, Greece, 2006, pp. 617-620.

\subsection{Future Work}

An important component to improve in both ADCs is the attenuation capacitor. The extraction tool * over-estimated the capacitance required for an accurate ADC and it seems to be at least $50 \mathrm{fF}$ too high based on measurements. This improvement would in turn improve the INL, DNL, SFDR, ENOB of the ADC and ensure that the tuning point for the ICGCUKAH is more towards the center of the tuning range instead of

\footnotetext{
${ }^{*}$ Cadence DIVA with the IBM $0.13 \mu \mathrm{m}$ kit 1.3.0.2.
} 
being the lowest possible capacitance for the tunable capacitor.

Another interesting improvement would be to increase the number of bits of the ADC and provide the tunable capacitor with more bits, (5-bits provides 32 capacitor values) and automate the tuning calibration. Initially when the ADC is powered on, a digital circuit determines the appropriate capacitance required for the attenuation capacitor.

There are a variety of further modifications that could improve the ADC operation. For example, when the comparator is not being used (eg. when the ADC is sampling the analog circuit), the comparator could be powered off which could further reduce the power dissipation. Also, perhaps a different flip-flop topology (with fewer transistors) would allow for a much smaller area for the digital circuitry. It may also be possible to redesign the ADC in a newer technology such as $90 \mathrm{~nm}$ or $65 \mathrm{~nm}$ CMOS to achieve an even more energy efficient ADC. 


\section{Bibliography}

[1] A. Raychowdhury, B. Paul, S. Bhunia, and K. Roy, "Computing with Sub-threshold Leakage: Device/Circuit/Architecture Co-Design for Ultralow-Power Sub-threshold Operation," IEEE transactions on Very Large Scale Integration (VLSI Systems), vol. 13, no. 11, pp. 1213-1224, November 2005.

[2] M. D. Scott, B. Boser, and K. Pister, "An Ultralow-Energy ADC for Smart Dust," IEEE Journal of Solid-State Circuits, vol. 38, no. 7, pp. 1123-1129, July 2003.

[3] J. Goes, N. Paulino, H. Pinto, R. Monteiro, B. Vaz, and A. Garcao, "Low-Power LowVoltage CMOS A/D Sigma-Delta Modulator for Bio-Potential Signals Driven by a Single-Phase Scheme," in IEEE Transactions on Circuits and Systems, vol. 52, no. 12, December 2005, pp 1058-1067.

[4] K. A. Townsend, J. Haslett, T. Tsang, M. El-Gamal, and K. Iniewski, "Recent Advances and Future Trends in Low Power Wireless Systems for Medical Applications," in Proceedings of the 9th International Database Engineering and Application Symposium, 2005.

[5] R. Amirtharajah and A. Chandrakasan, "Self Powered Signal Processing Using Vibration Based Power Generation," in IEEE Journal of Solid-State Circuits, vol. 33, no. 5, May 1998, pp. 687695.

[6] T. Starner, "Human Powered Wearable Computing," in IBM Systems Journal, vol. 35, no. 3, 1996, pp. 618-629.

[7] J. M. Rabaey, J. Ammer, T. Karalar, S. Li, B. Otis, M. Sheets, and T. Tuan, "Picoradios for Wireless Sensor Networks the Next Challenge in Ultra-Low Power Design," in IEEE Solid-State Circuits Conference, 2002.

[8] T. Doueski, T. Tsukahara, Y. Yoshida, F. Utsunomiya, and N. Hama, "A Batteryless Wireless System with MTCMOSSOI Circuit Technology," in IEEE Custom Integrated Circuits Conference, 2003, pp. 163-168.

[9] C. H.-I. Kim, H. Soeleman, and K. Roy, "Ultra Low Power DLMS Adaptive Filter for Hearing Aid Applications," IEEE transactions on Very Large Scale Integration (VLSI Systems), vol. 11, no. 6, pp. 1058-1067, December 2003.

[10] M. D. Scott, "An Ultra Low-Power ADC for Distributed Sensor Networks," Master's thesis, University of California at Berkeley, 2003.

[11] H. Yang and R. Sarpeshkar, "A Bio-Inspired Ultra-Energy-Efficient Analog-to-Digital Converter for Biomedical Applications," in IEEE Transactions on Circuits and Systems, vol. 53, no. 11, November 2005, pp. 2349-2356.

[12] J. Sauerbrey, D. Schmitt-Landsiedel, and R. Thewes, "A 0.5V, $1 \mu \mathrm{w}$ Successive Approximation ADC," IEEE Journal of Solid-State Circuits, vol. 38, no. 7, pp. 1261-1265, July 2003.

[13] L. S. Y. Wong, S. Hossain, A. Ta, J. Edvinsson, D. Rivas, and H. Naas, "A Very Low-Power CMOS Mixed-Signal IC for Implantable Pacemaker Applications," IEEE Journal of Solid-State Circuits, vol. 39, no. 12, pp. 2446-2456, December 2004. 
[14] Z. Fu and E. Culurciello, "An Ultra-Low Power Silicon-On Sapphire ADC for EnergyScavenging Sensors," in ISCAS '06. Proceedings of the 2006 International Symposium on Circuits and Systems, vol. 1, 2006, pp. 1511-1514.

[15] J. Nielsen and E. Bruun, "A Low-Power 10-bit Continuous-Time CMOS Sigma Delta AD Converter," in IEEE Transactions on Circuits and Systems, vol. 1, May 2004, pp. 174-178.

[16] C. A. V. de la Cruz, "A Switched Opamp to Comparator to Improve the Conversion Rate of Low Voltage Successive Approximation ADCs," Master's thesis, University of Puerto Rico, 2005 .

[17] R. Jacob Baker, CMOS: Circuit Design, Layout, and Simulation, 2nd ed. John Wiley and Sons, 2005.

[18] D. A. Johns and K. Martin, Analog Integrated Circuit Design. Wiley, 1997.

[19] R. Wang, "A Low Voltage Low Power 10-bit Pipeline ADC in 90nm CMOS Technology," Master's thesis, University of Toronto, 2004.

[20] K. K. Nair, "Low Power Analogue to Digital Converter for Power Management ICs," Master's thesis, University of Edinburgh, 2005.

[21] S. Gambini, "Low Voltage Analog to Digital Converter Design in 90nm CMOS," Master's thesis, University of California at Berkeley, 2007.

[22] A. Agarwal, "Low Power Current Mode ADC for CMOS Sensor IC," Master's thesis, Texas AM University, 2005.

[23] G. Bonfini, A. S. Brogna, C. Garbossa, L. Colombini, M. Bacci, S. Chicca, F. Bigongiari, N. C. Guerrini, and G. Ferri, "An Ultralow-Power Switched Opamp-Based 10-b Integrated ADC for Implantable Biomedical Applications," in IEEE Transactions on Circuits and Systems, vol. 51, no. 1, January 2004.

[24] H. Le, J. Singh, L. Hiremath, V. Mallapur, and A. Stojcevski, "Ultra-Low-Power VariableResolution Successive Approximation ADC for Biomedical Application," in Electronic Letters, vol. 41, no. 11, May 2005.

[25] R. Gregorian, CMOS Op-Amps and Comparators. New York, NY: John Wiley and Sons, 1999.

[26] Jan M. Rabaey and Anantha Chandrakasan and Borivoje Nikolic, Digital Integrated Circuits, 2nd ed. Prentice Hall Electronics and VLSI Series, 2003.

[27] C. Plett and J. Rogers, Radio Frequency Integrated Circuit Design. Artech House, 2003.

[28] Y. Nemirovsky, I. Brouk, and C. Jakobson, "1/f Noise in CMOS Transistors for Analog Applications," IEEE transactions on Electron Devices, vol. 48, no. 5, pp. 927-927, May 2001.

[29] K. Abdelhalim, L. MacEachern, and S. Mahmoud, "A Nanowatt ADC for Ultra-Low-Power Applications," in ISCAS '06. Proceedings of the 2006 International Symposium on Circuits and Systems, vol. 1, 2006, pp. 617-620.

[30] T. Anderson, "Optimum Control Logic For Successive Approximation A-C Converters," Computational Design, vol. 11, no. 7, pp. 81-86, July 1972.

[31] J. He and K. J. Turner, "Extended DILL: Digital Logic in LOTUS," University of Stirling, Tech. Rep., November 1997.

[32] E. Culurciello and A. Andreou, "An 8-bit, 1mW Successive Approximation ADC in SOI CMOS," in ISCAS '03. Proceedings of the 2003 International Symposium on Circuits and Systems, vol. 1, 2003, pp. 301-304.

[33] MAX1474, "Minature Electronically Trimmable Capacitor," Maxim Electronics, USA, Tech. Rep., 2001, available at http://datasheets.maxim-ic.com/en/ds/MAX1474.pdf. 
[34] AN283, "INL/DNL Measurements for High Speed ADCs," Maxim Electronics, USA, Tech. Rep., 2005, available at http://pdfserv.maxim-ic.com/en/an/AN283.pdf.

[35] Walt Kester, "ABCs of ADCs," National Semiconductor, USA, Tech. Rep., 2004, available at http://www.national.com/appinfo/adc/files/ABCs_of_ADCs.pdf.

[36] Nick Gray, "MT-003: Understand SINAD, ENOB, SNR, THD, THD + N, and SFDR so You Don't Get Lost in the Noise Floor," Analog Devices, Tech. Rep., 2005, available at http://www.spsc.tugraz.at/courses/msp/msp/data/MT-003_SINAD.pdf. 